\title{
Fermentation of indigestible carbohydrates by the gut microbiota
}

Citation for published version (APA):

Aguirre Morales, M. (2016). Fermentation of indigestible carbohydrates by the gut microbiota: one small step for a microbe, a giant leap for mankind? [Doctoral Thesis, Maastricht University]. https://doi.org/10.26481/dis.20160704ma

Document status and date:

Published: 01/01/2016

DOI:

10.26481/dis.20160704ma

Document Version:

Publisher's PDF, also known as Version of record

\section{Please check the document version of this publication:}

- A submitted manuscript is the version of the article upon submission and before peer-review. There can be important differences between the submitted version and the official published version of record.

People interested in the research are advised to contact the author for the final version of the publication, or visit the DOI to the publisher's website.

- The final author version and the galley proof are versions of the publication after peer review.

- The final published version features the final layout of the paper including the volume, issue and page numbers.

Link to publication

\footnotetext{
General rights rights.

- You may freely distribute the URL identifying the publication in the public portal. please follow below link for the End User Agreement:

www.umlib.nl/taverne-license

Take down policy

If you believe that this document breaches copyright please contact us at:

repository@maastrichtuniversity.nl

providing details and we will investigate your claim.
}

Copyright and moral rights for the publications made accessible in the public portal are retained by the authors and/or other copyright owners and it is a condition of accessing publications that users recognise and abide by the legal requirements associated with these

- Users may download and print one copy of any publication from the public portal for the purpose of private study or research.

- You may not further distribute the material or use it for any profit-making activity or commercial gain

If the publication is distributed under the terms of Article $25 \mathrm{fa}$ of the Dutch Copyright Act, indicated by the "Taverne" license above, 


\section{Fermentation of indigestible carbohydrates by the gut microbiota}

one small step for a microbe, a giant leap for mankind? 


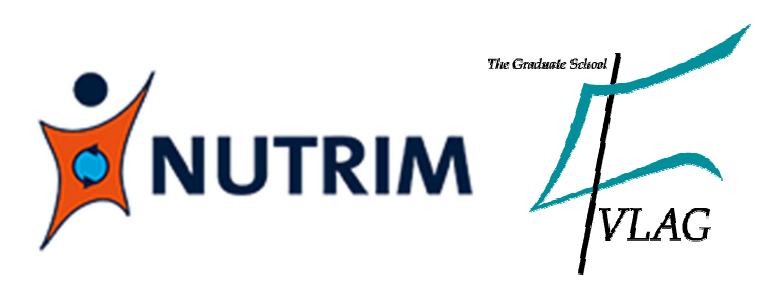

The research presented in this dissertation was conducted at NUTRIM School of Nutrition and Translational Research in Metabolism of Maastricht University which participates in the Graduate School VLAG (Food Technology, Agrobiotechnology, Nutrition and Health Sciences), accredited by the Royal Netherlands Academy of Arts and Sciences.

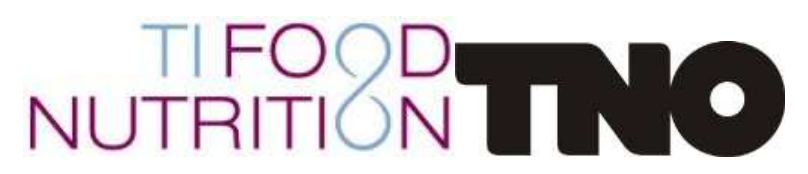

The studies presented in this thesis were performed within the framework of TI Food and Nutrition (TIFN). Financial support from TIFN (GH004; Wageningen, The Netherlands) and The Netherlands Organization for Applied Scientific research (TNO; Zeist, The Netherlands) is gratefully acknowledged.

Cover-design: Marisol Aguirre and Deivid J. Velasquez (www.behance.net/BelmontDesign)

Layout: Marisol Aguirre

Printed by: Proefschriftmaken.nl, The Netherlands

(C) Marisol Aguirre, Maastricht 2016

ISBN: 9789462953499 


\title{
Fermentation of indigestible carbohydrates by the gut microbiota \\ one small step for a microbe, a giant leap for mankind?
}

\author{
Dissertation \\ To obtain the degree of doctor at Maastricht University, on the authority of the \\ Rector Magnificus, \\ Prof. dr. L.L.G. Soete, \\ in accordance with the decision of the Board of Deans, \\ to be defended in public \\ On Monday $4^{\text {th }}$ of July 2016 at 14:00
}

by

Marisol Aguirre

Born in Palmira, Colombia, on May 30th 1986 


\section{Promotors:}

Prof. dr. F. Brouns

Prof. dr. K. Venema

\section{Assessment committee}

Prof. dr. W.H.M. Saris, Chair

Prof. dr. E.E. Blaak

Prof. dr. J. Knol, Wageningen University

dr. J. Penders

Prof. dr. M.Y Sanz Herranz, IATA, Valencia, Spain

Thesis Maastricht University

All rights reserved. No part of this thesis may be reproduced, distributed, stored in a retrieval system, or transmitted in any form or by any means without prior permission of the author. 


\section{Content}

Chapter 1 General introduction 7

Microorganisms 2015, 3, 213-235.

Genes Nutr. 2015, 10(4):472

Chapter 2 To pool or not to pool? Impact of the use of individual and pooled fecal samples for in vitro fermentation studies.

J Microbiol Methods.2014, 107C: 1-7.

Chapter 3 Evaluation of an optimal preparation of human standardized fecal inocula for in vitro fermentation studies J Microbiol Methods. 2015, 117: 78-84.

Chapter 4 Seeing is believing: a repeatable, robust and reproducible in vitro simulation of the human proximal colon

Chapter 5 Diet drives quick changes in the metabolic 107 activity and composition of human gut microbiota in a validated in vitro gut model Research in Microbiology. 2016, 167: 114125

Chapter 6 In vitro characterization of the impact of different substrates on metabolite production, energy extraction and composition of gut microbiota from lean and obese subjects

PLOS ONE. 2014, 9(11): e113864.

Chapter 7 The gut microbiota from lean and obese subjects contribute differently to the fermentation of arabinogalactan and inulin Submitted

Chapter 8 Dynamics of defined and complex microbial communities fermenting mucin in an in vitro simulation of the human gut

Chapter 9 General discussion

Eur J Epidemiol. 2015, 30:1067-1069

Submitted

Summary

Samenvatting

Valorization (submitted) 257

Acknowledgments $\quad 275$

$\begin{array}{ll}\text { About the author } & 279\end{array}$ 



\section{Chapter 1}

\section{General Introduction}

Published as:

Aguirre M, Venema K (2015) Does the Gut Microbiota Contribute to Obesity? Going beyond the Gut Feeling. Microorganisms 2015, 3, 213-235

Aguirre M, Venema K (2015) The art of targeting gut microbiota for tackling human obesity. Genes Nutr 2015, 10(4): 472 


\section{Obesity}

The adoption of a modern/Western type lifestyle, characterized by a high consumption of energy-dense foods and reduced physical activity, has been accompanied by the growth of obesity in developed and industrialized countries $[1,2]$. According to the World Health Organization (WHO), more than 1.9 billion adults (> 18 years old) were overweight in 2014. From this number, it was estimated that around 600 million were obese [3].

The long-held belief of considering obesity as mainly associated with an imbalance in energy consumed when compared to energy expenditure, seems to be incomplete given the recent mechanisms proposed to underlie obesity [1]. Namely, growing evidence suggests a less simplistic event which involves a combination of factors including: environment, genetics, diet and lifestyle, adipose tissue and systemic inflammation $[1,4,5]$. Moreover, the gut microbiota has been proposed as an environmental factor that plays a crucial role in obesity [1].

The human gut can be considered a bioreactor that harbors a complex ecosystem of microbes, collectively termed the microbiota, which is estimated to include at least $10^{14}$ cells $\mathrm{ml}^{-1}$ [6]. Nearly $1.5 \mathrm{~kg}$ of bacteria reside in the human gut [7] and approximately $50 \%$ of the wet-weight of fecal biomass in humans is estimated to come from bacterial cells [8]. Not surprisingly, the gut microbiota has been studied the most in adults [9]. It consists of a community of primarily Firmicutes, Bacteroidetes, Actinobacteria, Fusobacteria, Proteobacteria and Verrucomicrobia [9]. The combination of the proteins/enzymes encoded by their genomes (more than 5 million genes) yields additional molecules and grants special functions exceeding the host's own genetic potential by 2 orders of magnitude [10,11]. Interesting examples about the influence of the metabolic activity of the microbiota on humans include the observations by Hehemann et al. [12], who identified $\beta$ porphyranase, an enzyme previously found in the seaweed-associated bacterium Zobellia glactanivorans, in the genome of Bacteroides plebeius, a bacterium that has been only isolated in Japanese individuals. Strikingly, $\beta$-porphyranase confers the capacity to hydrolyze indigestible polysaccharides present in marine plants. Another interesting example includes the study in which it was found that African children could digest cellulose due to their unique gut microbiota [13].

Obesity, in terms of microbiota, is a complicated disequilibrium that presents many complications (Fig. 1). Chronic low-grade endotoxemia, modulation of secretion of gut-derived peptide hormones, regulation of active adipose tissue composition and increased energy harvest from host diet have been suggested as mechanisms through which the gut microbiota may contribute to obesity [14]. Still, the role of the gut microbiota in human obesity remains unclear. Different reasons may 
explain this lack of consensus. On the one hand, most studies have been done in rodent models which carry some disadvantages due to the differences in terms of gut microbiota composition, fermentation process (location, rates of digesta passage, etc) and dietary practices (coprophagia) [15,16]. On the other hand, studies in humans have shown a large inter-individual variation in the gut microbiota composition and the different methods used to analyze the bacteria together with involving participants with different backgrounds (food habits and ethnicity) constitute factors that influence the sometimes contradictory results found $[17,18]$. It is also because of such reasons that it is difficult to clearly provide a definition for a healthy microbiota. Nevertheless, diversity, richness and evenness in the composition of the gut bacterial community have been found to be altered or to have an effect in obese subjects [19]. Backhed et al. [6] observed that germ free mice remained lean when raised without microbiota despite their genetic predisposition to obesity. Interestingly, the conventionalization of mice with gut microbiota led to an improved absorption of monosaccharides. Findings also suggest that microbes colonizing a mucosal surface interfere in the formation of microvasculature, which suggests microbial regulation of angiogenesis [20].

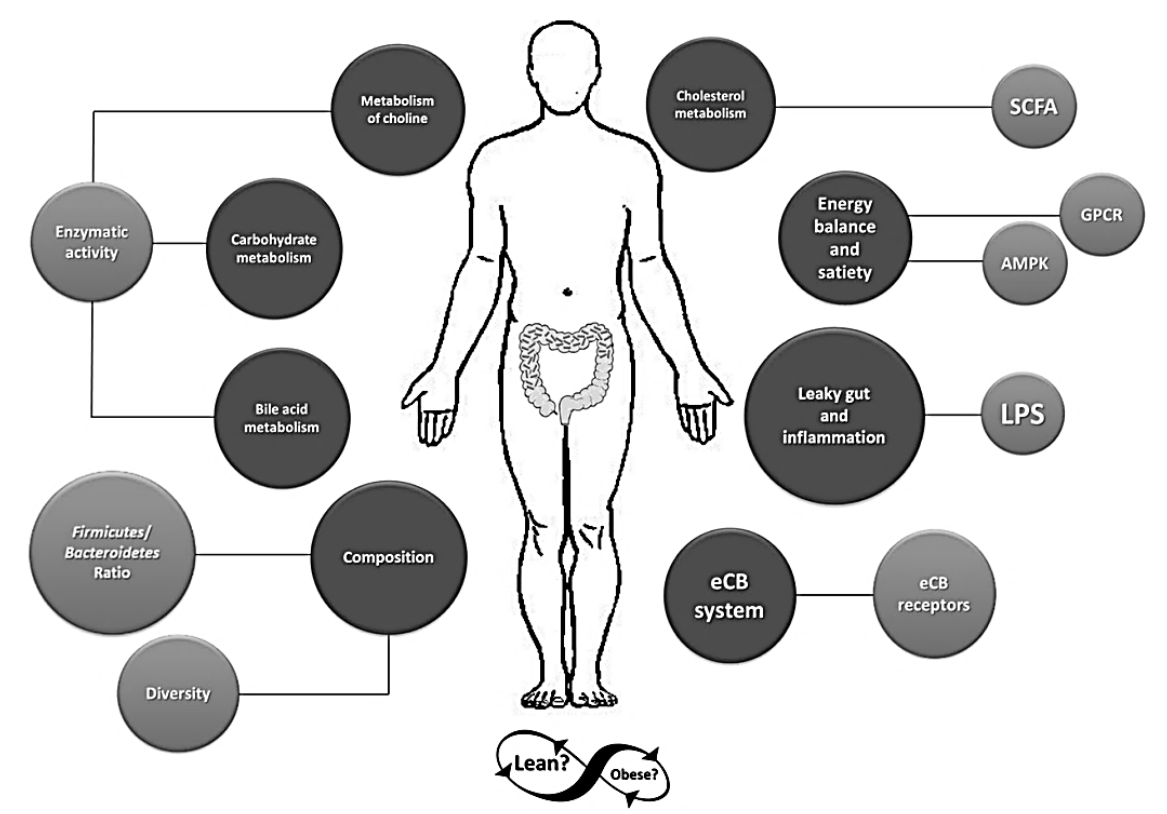

Fig. 1. The gut microbiota may lead to obesity by disturbing host homeostasis.

Diet is also believed to influence the composition and activity of the gut microbiota. For instance, increasing evidence shows the modulation of the gut 
bacterial members after following a high-fat diet, which is accompanied, in particular, with a reduction in bifidobacteria [21,22,23,24]. The high-fat diet has been shown to lead to an increase in gut permeability which influences lipopolysaccharide (LPS) plasma levels, potentially leading to inflammation [23]. Obesity could lead to an important number of metabolic diseases that include increased morbidity and mortality which implies, besides a detrimental quality of life, high health care costs [1]. Therefore, a better understanding of the interaction of diet, microbiota and host are fundamental in recommending lifestyle and therapeutic approaches to tackle obesity in humans.

\section{Dominant gut microbiota in obese individuals}

It has been suggested that the microbiota from obese individuals has an uncharacterized property that notably favors the balance towards Firmicutes when compared to Bacteroidetes [19]. Ley et al. [25] found a lower relative abundance of Bacteroidetes and a proportional increase in Firmicutes in obese mice. Such changes were observed to be independent from food consumption. However, differences in the abundance of these two phyla were also observed in individuals under a carbohydrate or fat restricted low calorie diet [19]. The increase in Bacteroidetes was correlated to weight loss in these subjects.

Others have not detected such a relationship in the proportion or ratio of these populations and obesity, or have shown the complete opposite results [26,27,28]. Possible reasons behind the contradictory observations could be attributed to differences among the subjects studied (age, diet, geographical origin) [29,30] and the study design (whether weight loss or weight gain were studied, or a comparison between obese and lean was carried out) which has a major impact on finding differences in the data. In addition, biases inherent to the techniques used to study the composition of the microbiota can contribute to contradictory observations [31].

Interestingly, molecular analysis of the gut microbiota in obese Indian individuals revealed a predominance in Bacteroides genus [32]. Furthermore, high archaeal densities together with high short-chain fatty acids (SCFA) levels were identified in these subjects. Therefore, as commonly referred to, the Firmicutes/Bacteroidetes ratio is likely to be just the tip of the iceberg.

Increasing evidence emphasize that different phyla (including both Firmicutes and Bacteroidetes) are composed of a wide variety of genera and species whose role in obesity has not been evaluated, promising future research [26,33]. For instance, Staphylococcus aureus (Firmicutes) have been associated with an obese phenotype [34]. However, also Halomonas, and Sphingomonas (Proteobacteria) have been 
found in higher abundances accompanied with low Bifidobacterium (Actinobacteria) numbers when compared to lean individuals [35].

It has also been suggested that obese individuals present an increased energy uptake mediated by an important mechanism involving interspecies hydrogen $\left(\mathrm{H}_{2}\right)$ transfer (e.g., $\mathrm{H}_{2}$ producing bacteria and $\mathrm{H}_{2}$ utilizing methanogens) [36]. Zhang et al. [36] proposed that such mechanism stimulates the fermentation of nondigestible carbohydrates and, therefore, an increased production of SCFA. However, as observed by Venema et al. [26], it remains unclear from such hypothesis how increased energy harvest can take place while there is loss of carbon.

Commensal mucosal bacteria interacting with the mucus layer may also have an effect on obesity [37]. Everard et al. [37] inversely correlated the abundance of $A$. muciniphila and obesity. The study clearly shows the importance of this mucin degrading bacteria in the regulation of the cross-talk between gut microbiota and the host. Their results provide good insight into the improvement in the metabolic profile in the host when this species is present as well as its role in controlling gut peptide secretion, inflammation and gut barrier function.

Recently, enrichment of the family Christensenellaceae has been found in lean individuals which, when transplanted to mice, have shown to promote a lean host phenotype and had an impact on the diversity of the community [38].

Finally, there is also the hypothesis that lower diversity in the gut microbiota may have an effect on satiety and eating behavior [39]. Studies have found that the gut microbial community from obese twins is less diverse when compared with lean twins [40]. In addition, antibiotic treatment studies indicate that there is an impact of long-term exposure of antibiotics on acquired obesity and weight gain $[41,42,43]$. In fact, antibiotics have been used in animals as growth-promoting agents prior to their ban by regulatory bodies [44].

\section{Contribution to energy balance and satiety}

In the context of energy balance and satiety, two pathways that may play an important role in obesity represent the potential interaction between host and the microbiota.

First, the regulation of adenosine monophosphate activated protein kinase (AMPK) which is a key enzyme that controls cellular energy [1]. The activation of AMPK stimulates the activity of several transcription factors that are crucial in the regulation of glucose, cholesterol and lipid metabolism, enhancing fatty acid oxidation [45]. Correspondingly, the down-regulated expression of AMPK by gut 
microbiota increases adipose tissue weight by inhibiting fatty-acid oxidation, which results in obesity $[46,47]$.

The second pathway is via the activation of important G-protein coupled receptors (GPCR) that are associated with glucose and lipid metabolism [45]. SCFA produced by fermentative bacteria may play a role as signaling molecules of such GPCR which may contribute to regulation of nutrient uptake and fat deposition (Figure 1). Acetate, propionate and butyrate have been found to be ligands for GPCR41 and GPCR43 (also known as free fatty acid receptors (FFAR)-3 and FFAR-2, respectively) [48,49]. However, their affinity differs: for GPCR43: acetate $=$ propionate $>$ butyrate, whilst for GPCR41: butyrate $=$ propionate $>$ acetate [45]. The activation of GPCR41 and GPCR43 may increase gut hormones such as glucagon-like peptide-1 (GLP-1) and peptide YY (PYY) [45]. GLP-1 stimulates insulin secretion which slows down gastric emptying and promotes satiety [2]. PYY secretion decelerates intestinal transit and suppresses gut motility, and in turn, food digestion and absorption of nutrients are up-regulated [2]. Furthermore, it has been found that PYY boosts the action of insulin on glucose absorption in adipose and muscle tissue [17,45]. Strikingly, energy expenditure may be also down-regulated by SCFA via GPCR41 by alternatively activating, at the ganglionic level, the sympathetic nervous system [50]. On the other hand, leptin expression, a hormone that increases energy metabolism and inhibits the feeling of hunger, has also been found to be stimulated by GPCR41 activation [51]. GPCR may also regulate the inhibition of lipolysis by a joint activation of hormone-sensitive lipase (HSL) and adipose triglyceride lipase (ATGL) [52]. The GPCR41 ligand butyric acid has been reported to inhibit lipolysis [53], however, it has not been demonstrated that the effects are directly mediated by GPCR41 activation [52]. Furthermore, GPCR43 has been found to inhibit lipolysis in murine adipocytes but its expression in human subcutaneous adipose tissue (SAT) has not been detected so far [52,54].

Some studies in knockout mice have shown conflicting results: on the one hand, the activity of GPCR41 and GPCR43 receptors have been found beneficial in regard to metabolic diseases and obesity, while others have proposed that their inhibition could also tackle the consequences of excess of energy intake [9]. For instance, in the study from Kimura et al. [55] the role of GPCR43 in fat storage was shown in mice. The authors observed that GPCR43-overexpressing mice were protected against obesity whilst deficient mice were obese when fed with a normal diet. However, Bjursell et al. [56] showed that GPCR43 deficient mice had an increased energy expenditure, lower body fat mass and improved insulin sensitivity under a high-fat diet showing that the deficiency of GPCR43 protected from obesity. 


\section{Experimental evidence on the influence of gut microbiota in the development of obesity}

Experimental models used to try to elucidate the role of gut microbiota in obesity include in vitro systems, animal models and humans. Human trials are considered as the golden standard. Still, the use of other approaches is prioritized before undertaking sometimes rather invasive and costly human interventions. Therefore, animal models have been considered as an ethically more acceptable, cheaper technique than human studies. Yet, these models do also include certain limitations to fully represent the complexity of a human being. For instance, despite the fact that it has been found that mice and humans share microbes from the main phyla, numerous bacterial genera and species from one are not detected in the other and vice versa [25,57]. Therefore, even though results from studies including native communities in mice are interesting, there is a high possibility that the animal's genotype strongly influences such results [57]. In order to overcome such bias, improved animal [57] and in vitro models [58] in which human fecal transplantation is performed have been developed and, even with the implicit limitation of not directly studying a human being as such, they represent a viable alternative for trying to elucidate the complex role of the gut microbiota in human health. Due to the scope of this thesis, only the evidence derived from in vitro studies about the potential role that the microbiota plays on human obesity is discussed in detail below:

\section{Evidence from in vitro studies}

Experiments involving in vitro fermentations have helped to simulate an ample number of different conditions such as age, diseases and disorders [59]. In vitro models closely mimicking the microbial metabolism in the human intestine can be used to get further insight in the complex mechanistic processes mediated by the gut microbiota. Hence, an in vitro study offers the great opportunity to examine microbe-microbe and microbe-substrate interactions in depth, by carefully controlling all variables and avoiding host derived interactions. However, findings need to be further validated in studies performed in either animals or humans.

There have been a limited number of in vitro reports investigating the differences of lean and obese microbiota. Few studies have provided evidence of the plasticity of the human gut microbiota in relation to dietary interventions. For instance, Payne et al. [91] evaluated three different Western dietary trends: high, normal and low energy reflecting obese, normal and anorectic dietary intakes in microbiota from obese and normal weight children. Their results demonstrate a metabolic adaptation of the microbiota in response to the different nutrient loads together 
with a reorganization of the structure of the bacterial community. Moreover, our own recent studies add to knowledge by suggesting that not all substrates are fermented in an identical manner by the gut microbiota, as clearly shown by the different measurements of SCFA and branched-chain fatty acids (BCFA) produced by lean and obese microbiota pointing to the possible implications in energy extraction if similar effects happen in vivo [60,61]. On the other hand, Yang et al. [62] observed non statistically significant differences in the microbiota activity from obese subjects when compared to lean after the in vitro fermentation with different dietary fibers but small differences in propionate and butyrate production were found. Nevertheless, different in vitro fermentation patterns between lean and obese microbiota have not been found by others. Sarbini et al. [63] studied the fermentation of $\alpha$-gluco-oligosaccharides and inulin and observed that they produced similar effects on bacterial population and metabolic activity in both lean and obese microbiotas. The same effect was observed by the authors when fermenting dextrans of different molecular weights [64].

Some other interesting studies such as the ones from Bussolo de Souza et al. [60] and Condezo-Hoyos et al. [65] observed clear differences in lean and obese subjects at the compositional level when studying the effects of in vitro fermentation of fibers from cassava bagasse and apple cultivars, respectively. Cassava bagasse is a by-product from starch production and cassava flour. Bussolo de Souza et al. [60] observed that cassava could modulate the microbiota composition from lean and obese individuals. The obese microbiota, in particular, became similar to the lean composition after the $72 \mathrm{~h}$ fermentation experiments which gave a nice indication of the improvement of the community's health. It is noteworthy that acetate production was higher in the obese microbiota, and due to the role of acetate in lipogenesis, the authors therefore speculate that such increase could be not "protective" against lipogenesis and further research is needed. Condezo-Hoyos et al. [65] observed an inverse trend in the proportion of Firmicutes and Bacteroidetes in feces from obese and lean. As explained by the authors, such differences may be the result of a complex and highly specific microbial ecosystem characteristic from subjects. Interestingly, these authors also found that after the administration of the fibers of different apple cultivars, the microbiota composition from obese mice tended to be similar to the lean controls.

Due to the conflicting pieces of evidence from in vitro data, animal models and human interventions it is important to understand and reduce the inconsistencies in the current scientific data. Still, though scarce, there are some in vitro studies that after investigating the influence of diet on microbes and optimal health have stressed the gut microbiota as a potential therapeutic treatment to tackle obesity. 


\section{Strategies to manipulate gut microbiota in obesity}

There are many promising strategies to manipulate the gut microbiota. In one of our reviews [66] the use of antimicrobials, probiotics, fecal microbial therapy, prebiotics and diet was highlighted (Fig. 2). However, for the purpose of this thesis it is important to focus the discussion on the impact of fecal microbial therapy (focusing mainly on synthetic fecal transplantations -SFT), prebiotics and diet.

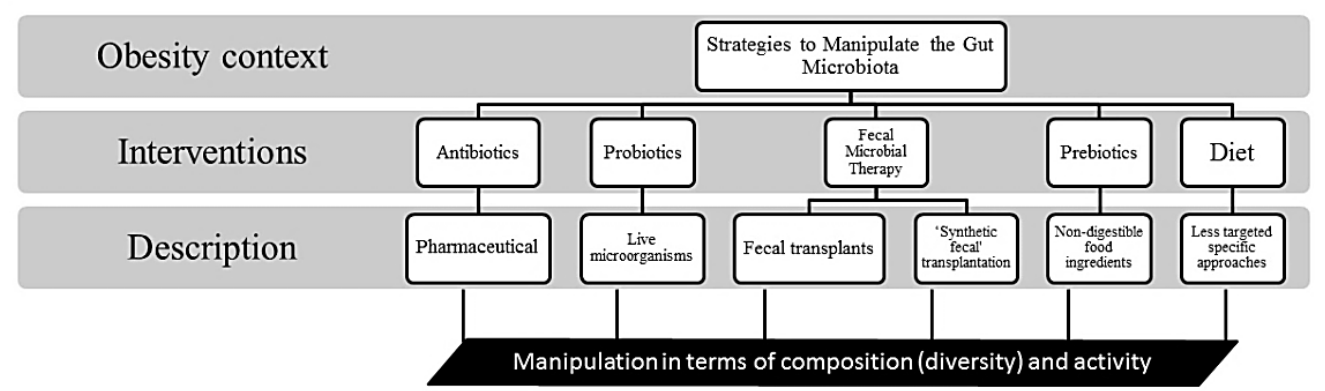

Fig. 2. Strategies to manipulate the gut microbiota in the context of obesity.

\section{Fecal microbial therapy}

Fecal matter has been traditionally considered a waste. Yet, the potential of fecal microbial therapies demonstrate the value of a fecal donation [67]. Currently, there are two innovative and non-invasive ways of fecal microbial therapy with a promising future in treating or preventing obesity: fecal microbiota transplantation (FMT) and (SFT).

In SFT, well characterized strains derived and cultured from a fecal donation from a healthy individual are transplanted to a recipient [67]. The strains are grown in cultures (defined communities that aim to be representative to a native fecal microbiota) and are administered in a suspension to the recipient [67,68]. This type of fecal substitute has shown to be a feasible and effective alternative to the use of the transplantation of real feces from a donor [69], which seems to overcome the limitations from FMT in terms of transmission of a donor's infection, and patient acceptance. As explained by Petrof et al. [69] the benefits from using a synthetic transplant over a conventional transplant are that the composition of the mix of bacteria to be transplanted is known which guarantees: i) an enhanced safety profile: the reduced contamination of potential pathogens including viruses, while the bacteria included are selected based on their sensitivity to antimicrobials; and ii) reproducibility: the preparation could be replicated in case of future treatment in a chemostat allowing the species to grow in a continuous culture under conditions 
resembling the gut without affecting its stability [67,69]. The pilot study from Petrof et al. [69] in which they evaluated the use of a synthetic stool comprising 33 isolates recovered from the fecal sample from a healthy donor seems promising for the treatment of Clostridium difficile infection. Interestingly, the authors also observed that the transplanted bacteria seemed to stably colonize the colon which offers an advantage over the consumption of probiotics due to their transient effect. However, conclusions about the potential effects of SFT cannot be easily drawn from the study due to the small patient population studied $(n=2)$. Therefore, rigorous clinical trials are also needed to study the utility, effectiveness and safety of SFT [67], as well as its potential as a therapy for obesity. Furthermore, a perceived difficulty of this technique comes from the experience that the microbial ecosystem from the human gut is difficult to culture. Yet, Petrof et al. [68] allude to recent technological advances to make progress in this area.

\section{Prebiotics}

The so-called "Western" diet is characterized by an unbalanced high content of refined carbohydrates when compared to other diets from the early human history, or those typical for developing countries [70]. The potential of prebiotics to reduce food intake, fat-mass development and body weight was demonstrated more than 10 years ago by changing the gut microbiota (with prebiotics such as inulin and oligofructose) and the cross-talk between the brain and gut microbiota [71,72,73]. Therefore, the future of prebiotics use to decrease the incidence and morbidity of obesity in humans is an interesting field to explore.

Prebiotics are defined as "nondigestible food ingredient that beneficially affects the host by selectively stimulating the growth and/or activity of one or a limited number of bacteria in the colon, and thus improving host health" [74]. Prebiotic treatment offers a wide range of benefits. It does not only promote the growth of specific bacteria but also inhibits the growth of certain other groups. Such effect was observed for instance by Everard et al. [75] in ob/ob mice after prebiotic treatment. The extensive gut microbiota analysis identified a catalogue of more than 100 operational taxonomic units (OTUs) that were different after the administration of the prebiotic feeding. Furthermore, fold changes (increase/decrease) of certain bacteria of more than 10 fold were observed in some cases.

The administration of prebiotics also stimulates the production of SCFA which may modulate the secretion of gut peptides $[48,50,51,54]$. Prebiotics may exert a satietogenic effect as demonstrated and confirmed in studies with mice in which the effects of acetate on production of appetite suppression were shown, even in 
absence of increased levels of GLP-1 and PYY [76]. In humans, the satietogenic effects of prebiotics such as oligofructose have also been shown [77,78].

Some data suggests that prebiotics also have the potential of enhancing the gut barrier which consequently improves metabolic disorders [57]. Cani et al. [79] observed that prebiotic (oligofructose) consumption in mice stimulated the production of GLP-2 which, at the same time, may lower plasma LPS, enhancing mucosal barrier function by improving tight junctions. As described by the authors, a wide range of specific tight-junction proteins controls gut permeability. ZO-1 and occludin proteins, in particular, have been considered as key markers of tightjunction integrity. Furthermore, oligofructose also stimulated the growth of Bifidobacterium spp which was also linked to a reduction in gut permeability. In addition, an increased production of SCFA is thought to be characteristic of the obese microbiome and it has been paradoxically observed to be reversed with the use of prebiotics [80].

The mechanisms through which prebiotics act have been suggested to be dosedependent [81]. Therefore, the full assessment of the therapeutic potential of prebiotics for treating obesity needs further study.

\section{Diet}

A high diverse microbiota is believed to be more resistant to changes when compared to less diverse communities [82]. As explained by Alcock et al. [39], the competing groups from a diverse microbiota may invest resources in counteracting and cooperating with each rather than manipulating the host.

Diet in general has been shown to exert an important impact on the gut microbiota regardless of other variables such as sanitation, ethnicity and climate [46]. For instance, a study comparing the composition of the microbiota from omnivores, carnivores and herbivores (in mammalian species and humans) revealed a separation of carnivores and omnivores from herbivores [83]. The authors emphasized the relevance of diet in establishing this differentiation among the analyzed communities. Another study correlated a long-term diet with gut microbial enterotypes [84]. The enterotype concept consists of clustering the gut bacterial community in three different consortia: Bacteroides-, Prevotella- or Ruminococcus-enriched, respectively. The Bacteroides enterotype has been found in subjects with a diet rich in animal fat, protein and saturated fats, whereas a diet low in these dietary components but rich in carbohydrates and simple sugars has been associated with the Prevotella enterotype [84].

Therefore, it is of great interest to identify dietary compounds for the reestablishment of the disturbed balance of the microbiota from obese subjects as a potential alternative to treat obesity. So far, these dietary compounds have been 
restricted to carbohydrates. However, more general approaches using the combination of weight-loss, weight maintenance diets and diets supplemented in prebiotic content have been also studied in humans. Some of these dietary interventions show that there is a very strong individual variation in responses $[85,86]$. Salonen et al. [85] attributed only $10 \%$ of the changes in the microbiota composition to diet after the study of obese subjects following four different fully controlled diets in a weight loss program.

Earlier, it was estimated that approximately $57 \%$ of the total structure (i.e., composition) of the gut microbiota could be explained by dietary changes, while some $12 \%$ could be attributed to host genetics [29]. However, this has been observed in studies with mice. As explained by Salonen et al. [85], the differences in their outcome and such reference value could be attributed to the fact that there is smaller total variance in the mice microbiota due to a better control of environmental and genetic variables in these animals. Therefore, it has been suggested to stratify human individuals into responders and non-responders based on their gut microbiota as previously proposed in fecal microbial transplantations.

Another factor to consider is that highly targeted specific dietary approaches can potentially affect the diversity of the gut microbial community and promote a dysbalance. For instance, the findings from Salonen et al. [85] provide an indication that a specific prebiotic may elicit the highly targeted dominance of certain groups, affecting the natural balance. Therefore, the promotion of a diverse community is suggested to be accomplished by providing complex or multiple substrates [85].

Compared to animal data, the data from human dietary interventions are less conclusive, which could be due to the fact that the high degree of variation in individuals makes it hard to find a "normal" response, or normal/healthy microbiota composition for that matter. This represents considerable frustration in nutritional studies which bears two questions: i) is there any strategy that could be applied to understand better this individuality?; and, ii) since variation has been considered a standard noise, can it really be handled or does it preclude to identify any effect on the microbiota composition? Furthermore, an important aspect to consider in dietary studies is the choice of its design which will make a major impact on finding statistically differences in the data.

\section{Outline of the thesis}

As described above, the potential role of gut microbiota in obesity has emerged as a novel subject in weight management research. 
Therefore, this work aimed to:

1. Contribute to know what the impact of the gut microbiota is in obesity when fermenting indigestible dietary compounds.

2. Elucidate the plasticity of this microbiota to manipulation and potential modification of an obese gut phenotype.

As such, this study set a precedent about how changes in the composition and activity of the microbiota from lean and obese subjects were during an in vitro fermentation process. Previous studies evaluated metabolites produced by colonic bacteria in feces from both type of donors. However, measurement of final content of short-chain fatty acids in feces has been considered an inaccurate prediction since at least $95 \%$ of these acids are absorbed in the gut. In contrast, in vitro fermentation using a fecal inoculum from this group of volunteers is a non-invasive alternative which allows a more complete following up through time about the different responses from the microbiota. Furthermore, in vitro testing about the impact of the gut microbiota on human obesity has been poorly performed in this field. Thus, use was made of a very well-known and validated in vitro system that simulates the proximal colon (TIM-2) to keep detailed track of possible compositional and functional disturbances caused by different fermentable substrates (Fig.3).
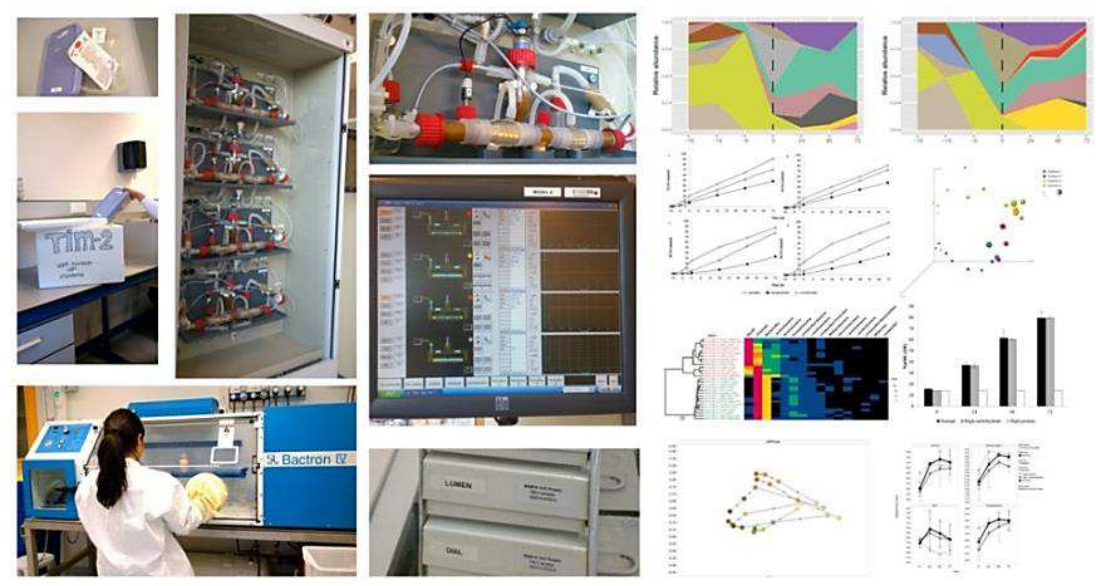

Fig. 3. Approach taken in this thesis: in vitro model of the large intestine TIM-2

The TIM-2 system is equipped with sensors to control $\mathrm{pH}$ (5.8), temperature (37 ${ }^{\circ} \mathrm{C}$ ) and volume (120 ml) of the fecal suspension studied (lumen). Such suspension is derived from fecal donations (from volunteers complying specific selection 
criteria) and is prepared under strict anaerobic conditions. The TIM-2 system includes a unique dialysis system which consists of a dialysate preparation running through a dialysis membrane which is placed inside the lumen in the model. This dialysis system contributes to maintain the physiological concentration of small molecules such as short-chain fatty acids (SCFA; 80-120 mM) and prevents their accumulation and therefore, reduces microbial death due to built-up of metabolites [87]. Furthermore, this dialysis system also contributes to maintain the concentration of electrolytes [88]. The TIM-2 system also incorporates four compartments with flexible walls which allows to recreate peristaltic movements at regular intervals. Such movements are computer controlled and mixes the luminal content avoiding separation of solids and fluids as commonly observed in other fermentors [89]. The model is kept anaerobical by flushing with gaseous nitrogen and the microbiota is fed through a feeding syringe at a controlled rate. All these advanced features have been shown to allow the growth of a highly complex, stable and dense $\left(\sim 10^{11} \mathrm{CFU} / \mathrm{ml}\right)$ active microbiota [90] and have demonstrated the sensitivity of the TIM-2 system for reproducibly detecting changes in the composition and activity of the bacteria under strictly controlled study designs [61]. 
The contribution of the different chapters to the aims of the present thesis and their context on the study of obesity is shown in Fig. 4.

This thesis starts with a set of studies in chapters 2-5 evaluating specific methodological challenges related with: i) the optimization of the preparation of inocula for in vitro fermentation studies, as well as ii) testing the suitability of the TIM-2 system as a tool to be used for the follow-up of the response of the microbiota to changes to dietary compounds. Specifically, in chapter $\mathbf{2}$, comparisons of the compositional and metabolic differences of the fermentation of a standard diet by the microbiota from different donors and a pool of prepared from them were made. Chapter 3 describes an optimal method for the preparation of human gut microbiota for fermentation studies which resembles metabolically and compositionally the closest to the reference (freshly collected microbiota). The focus of chapter 4 is the evaluation of the TIM-2 system as a sensitive tool to reproducibly show the diet-microbe interaction at metabolic, compositionally and gene level. Lastly, chapter 5 shows how the fermentation profiles change and how fast it is possible to detect the response from the microbiota after providing a diet with modified carbohydrate/protein ratios in TIM-2. In chapters 6 and 7 we proceeded with the evaluation of the differences on the fermentation profiles from microbiota derived from lean and obese individuals. In these studies we (separately) tested the fermentation of a wide range of prebiotics: galactooligosaccharides (GOS), lactulose, apple fiber, sugar beet pectin (chapter 6), arabinogalactan and inulin (chapter 7). The energy extracted from the different prebiotics fermented was estimated from the short-chain fatty acids produced and compositional changes of both microbiotas were also followed. In chapter 8 we explored: i) the impact of the addition of mucin in the development of complex (lean and obese) and defined (14-members comprising the 5 most dominant phyla in the healthy human gut) communities in TIM-2, as well as ii) how the functional (metabolic and gene-expression) and compositional performance of the defined community was with respect to the complex community. In chapter 9, the main findings of this thesis are discussed and are put into a broader perspective. 
carbohydrate and protein on the gut microbiota

and

how fast the response is

Diet
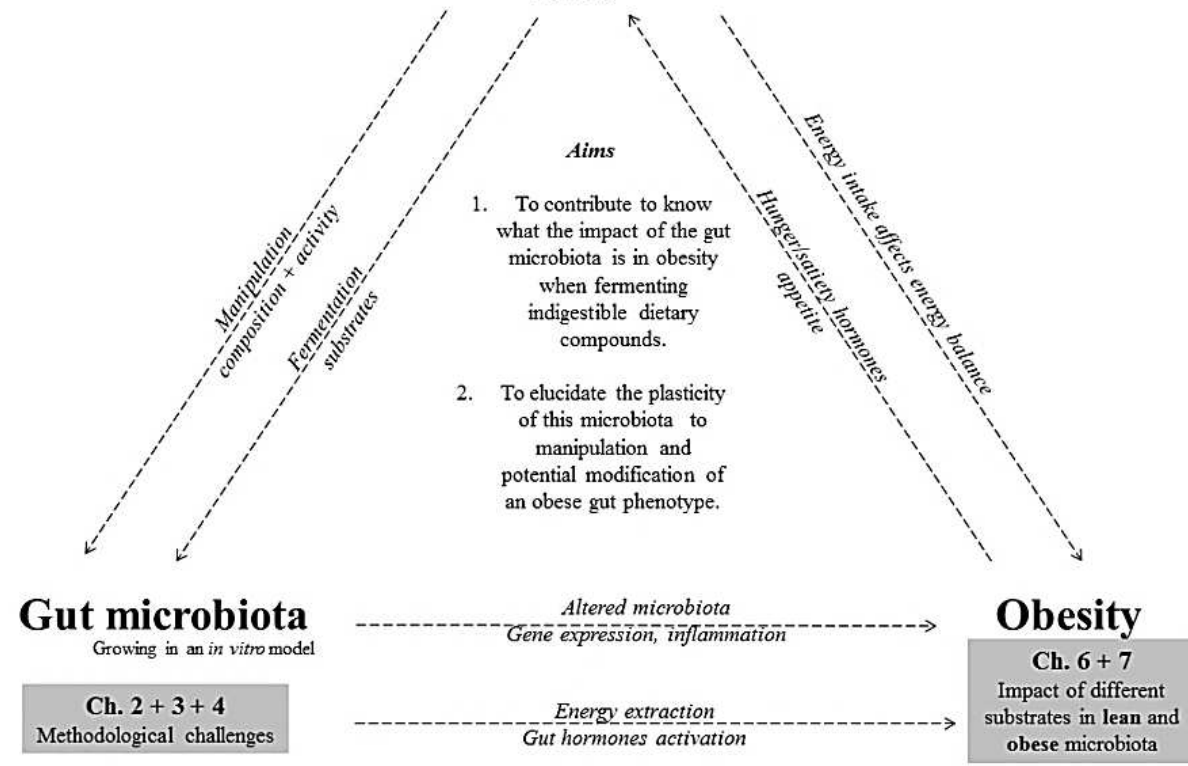

Fig. 4. Contribution of the different chapters to the aims of this thesis. 


\section{References}

1. Kotzampassi K, Giamarellos-Bourboulis EJ, Stavrou G (2014) Obesity as a consequence of gut bacteria and diet interactions. ISRN Obes 2014: 651895.

2. Conterno L, Fava F, Viola R, Tuohy KM (2011) Obesity and the gut microbiota: does up-regulating colonic fermentation protect against obesity and metabolic disease? Genes Nutr 6: 241-260.

3. World Health Organization (WHO) (2015) Obesity and overweight. Fact sheet No.311 http://www.who.int/mediacentre/factsheets/fs311/en/

4. Moran C, Shanahan F (2014) Gut microbiota and obesity: Role in aetiology and potential therapeutic target. Best Pract Res Clin Gastroenterol 28: 585-597.

5. Guida S, Venema K (2015) Gut microbiota and obesity: Involvement of the adipose tissue. J Funct Foods 14: 407-423.

6. Backhed F, Ding H, Wang T, Hooper LV, Koh GY, et al. (2004) The gut microbiota as an environmental factor that regulates fat storage. Proc Natl Acad Sci U S A 101: 15718-15723.

7. Nicholson JK, Holmes E, Wilson ID (2005) Gut microorganisms, mammalian metabolism and personalized health care. Nat Rev Microbiol 3: 431-438.

8. Zhao L (2013) The gut microbiota and obesity: from correlation to causality. Nat Rev Micro 11: 639-647.

9. Brahe LK, Astrup A, Larsen LH (2013) Is butyrate the link between diet, intestinal microbiota and obesity-related metabolic diseases? Obes Rev 14: 950-959.

10. Hernandez E, Bargiela R, Diez MS, Friedrichs A, Perez-Cobas AE, et al. (2013) Functional consequences of microbial shifts in the human gastrointestinal tract linked to antibiotic treatment and obesity. Gut Microbes 4: 306-315.

11. Sommer F, Backhed F (2013) The gut microbiota--masters of host development and physiology. Nat Rev Microbiol 11: 227-238.

12. Hehemann J-H, Correc G, Barbeyron T, Helbert W, Czjzek M, et al. (2010) Transfer of carbohydrate-active enzymes from marine bacteria to Japanese gut microbiota. Nature 464: 908-912.

13. De Filippo C, Cavalieri D, Di Paola M, Ramazzotti M, Poullet JB, et al. (2010) Impact of diet in shaping gut microbiota revealed by a comparative study in children from Europe and rural Africa. PNAS 107: 14691-14696.

14. Musso G, Gambino R, Cassader M (2010) Obesity, diabetes, and gut microbiota: the hygiene hypothesis expanded? Diabetes Care 33: 2277-2284.

15. Heinritz SN, Mosenthin R, Weiss E (2013) Use of pigs as a potential model for research into dietary modulation of the human gut microbiota. Nutr Res Rev 26: 191-209.

16. Houpt KA, Houpt TR, Pond WG (1979) The pig as a model for the study of obesity and of control of food intake: a review. Yale J Biol Med 52: 307-329. 
17. Moreno-Indias I, Cardona F, Tinahones FJ, Queipo-Ortuno MI (2014) Impact of the gut microbiota on the development of obesity and type 2 diabetes mellitus. Front Microbiol 5: 190.

18. Tremaroli V, Backhed $\mathrm{F}$ (2012) Functional interactions between the gut microbiota and host metabolism. Nature 489: 242-249.

19. Ley RE, Turnbaugh PJ, Klein S, Gordon JI (2006) Microbial ecology: human gut microbes associated with obesity. Nature 444: 1022-1023.

20. Stappenbeck TS, Hooper LV, Gordon JI (2002) Developmental regulation of intestinal angiogenesis by indigenous microbes via Paneth cells. Proceedings of the National Academy of Sciences 99: 15451-15455.

21. Turnbaugh PJ, Backhed F, Fulton L, Gordon JI (2008) Diet-induced obesity is linked to marked but reversible alterations in the mouse distal gut microbiome. Cell Host Microbe 3: 213-223.

22. Cani PD, Delzenne NM (2007) Gut microflora as a target for energy and metabolic homeostasis. Curr Opin Clin Nutr Metab Care 10: 729-734.

23. Cani PD, Hoste S, Guiot Y, Delzenne NM (2007) Dietary non-digestible carbohydrates promote L-cell differentiation in the proximal colon of rats. $\mathrm{Br} J$ Nutr 98: 32-37.

24. Cani PD, Bibiloni R, Knauf C, Waget A, Neyrinck AM, et al. (2008) Changes in gut microbiota control metabolic endotoxemia-induced inflammation in highfat diet-induced obesity and diabetes in mice. Diabetes 57: 1470-1481.

25. Ley RE, Backhed F, Turnbaugh P, Lozupone CA, Knight RD, et al. (2005) Obesity alters gut microbial ecology. Proc Natl Acad Sci U S A 102: 11070-11075.

26. Venema K (2010) Role of gut microbiota in the control of energy and carbohydrate metabolism. Curr Opin Clin Nutr Metab Care 13: 432-438.

27. Duncan SH, Lobley GE, Holtrop G, Ince J, Johnstone AM, et al. (2008) Human colonic microbiota associated with diet, obesity and weight loss. Int $\mathrm{J}$ Obes (Lond) 32: 1720-1724.

28. Schwiertz A, Taras D, Schafer K, Beijer S, Bos NA, et al. (2010) Microbiota and SCFA in lean and overweight healthy subjects. Obesity (Silver Spring) 18: 190-195.

29. Zhang C, Zhang M, Wang S, Han R, Cao Y, et al. (2010) Interactions between gut microbiota, host genetics and diet relevant to development of metabolic syndromes in mice. ISME J 4: 232-241.

30. Ottman N, Smidt H, de Vos WM, Belzer C (2012) The function of our microbiota: who is out there and what do they do? Front Cell Infect Microbiol 2: 104.

31. Hugon P, Lagier JC, Robert C, Lepolard C, Papazian L, et al. (2013) Molecular studies neglect apparently gram-negative populations in the human gut microbiota. J Clin Microbiol 51: 3286-3293. 
32. Patil DP, Dhotre DP, Chavan SG, Sultan A, Jain DS, et al. (2012) Molecular analysis of gut microbiota in obesity among Indian individuals. J Biosci 37: 647-657.

33. Hoyles L, McCartney AL (2009) What do we mean when we refer to Bacteroidetes populations in the human gastrointestinal microbiota? FEMS Microbiol Lett 299: 175-183.

34. Kalliomaki M, Collado MC, Salminen S, Isolauri E (2008) Early differences in fecal microbiota composition in children may predict overweight. Am J Clin Nutr 87: 534-538.

35. Waldram A, Holmes E, Wang Y, Rantalainen M, Wilson ID, et al. (2009) Topdown systems biology modeling of host metabotype-microbiome associations in obese rodents. J Proteome Res 8: 2361-2375.

36. Zhang H, DiBaise JK, Zuccolo A, Kudrna D, Braidotti M, et al. (2009) Human gut microbiota in obesity and after gastric bypass. Proc Natl Acad Sci U S A 106: 2365-2370.

37. Everard A, Belzer C, Geurts L, Ouwerkerk JP, Druart C, et al. (2013) Cross-talk between Akkermansia muciniphila and intestinal epithelium controls dietinduced obesity. Proc Natl Acad Sci U S A 110: 9066-9071.

38. Goodrich JK, Waters JL, Poole AC, Sutter JL, Koren O, et al. (2014) Human genetics shape the gut microbiome. Cell 159: 789-799.

39. Alcock J, Maley CC, Aktipis CA (2014) Is eating behavior manipulated by the gastrointestinal microbiota? Evolutionary pressures and potential mechanisms. BioEssays 36: 940-949.

40. Turnbaugh PJ, Hamady M, Yatsunenko T, Cantarel BL, Duncan A, et al. (2009) A core gut microbiome in obese and lean twins. Nature 457: 480-484.

41. Ajslev TA, Andersen CS, Gamborg M, Sorensen TI, Jess T (2011) Childhood overweight after establishment of the gut microbiota: the role of delivery mode, pre-pregnancy weight and early administration of antibiotics. Int $J$ Obes (Lond) 35: 522-529.

42. Thuny F, Richet H, Casalta JP, Angelakis E, Habib G, et al. (2010) Vancomycin treatment of infective endocarditis is linked with recently acquired obesity. PLoS One 5: e9074.

43. Angelakis E, Armougom F, Million M, Raoult D (2012) The relationship between gut microbiota and weight gain in humans. Future Microbiol 7: 91-109.

44. Burch DG (1996) Is it time to ban all antibiotics as animal growth-promoting agents? Lancet 348: 1455; author reply 1455-1456.

45. den Besten G, van Eunen K, Groen AK, Venema K, Reijngoud DJ, et al. (2013) The role of short-chain fatty acids in the interplay between diet, gut microbiota, and host energy metabolism. J Lipid Res 54: 2325-2340.

46. Chen J, He X, Huang J (2014) Diet effects in gut microbiome and obesity. J Food Sci 79: R442-451. 
47. Backhed F, Manchester JK, Semenkovich CF, Gordon JI (2007) Mechanisms underlying the resistance to diet-induced obesity in germ-free mice. Proc Natl Acad Sci U S A 104: 979-984.

48. Brown AJ, Goldsworthy SM, Barnes AA, Eilert MM, Tcheang L, et al. (2003) The Orphan G protein-coupled receptors GPR41 and GPR43 are activated by propionate and other short chain carboxylic acids. J Biol Chem 278: 1131211319.

49. Le Poul E, Loison C, Struyf S, Springael JY, Lannoy V, et al. (2003) Functional characterization of human receptors for short chain fatty acids and their role in polymorphonuclear cell activation. J Biol Chem 278: 25481-25489.

50. Kimura I, Inoue D, Maeda T, Hara T, Ichimura A, et al. (2011) Short-chain fatty acids and ketones directly regulate sympathetic nervous system via $\mathrm{G}$ proteincoupled receptor 41 (GPR41). Proc Natl Acad Sci U S A 108: 8030-8035.

51. Samuel BS, Shaito A, Motoike T, Rey FE, Backhed F, et al. (2008) Effects of the gut microbiota on host adiposity are modulated by the short-chain fatty-acid binding G protein-coupled receptor, Gpr41. Proc Natl Acad Sci U S A 105: 16767-16772.

52. Amisten S, Neville M, Hawkes R, Persaud SJ, Karpe F, et al. (2014) An atlas of Gprotein coupled receptor expression and function in human subcutaneous adipose tissue. Pharmacol Ther 146: 61-93.

53. Ohira H, Fujioka Y, Katagiri C, Mamoto R, Aoyama-Ishikawa M, et al. (2013) Butyrate attenuates inflammation and lipolysis generated by the interaction of adipocytes and macrophages. $J$ Atheroscler Thromb 20: 425-442.

54. Ge H, Li X, Weiszmann J, Wang P, Baribault H, et al. (2008) Activation of G protein-coupled receptor 43 in adipocytes leads to inhibition of lipolysis and suppression of plasma free fatty acids. Endocrinology 149: 4519-4526.

55. Kimura I, Ozawa K, Inoue D, Imamura T, Kimura K, et al. (2013) The gut microbiota suppresses insulin-mediated fat accumulation via the short-chain fatty acid receptor GPR43. Nat Commun 4: 1829.

56. Bjursell M, Admyre T, Goransson M, Marley AE, Smith DM, et al. (2011) Improved glucose control and reduced body fat mass in free fatty acid receptor 2-deficient mice fed a high-fat diet. Am J Physiol Endocrinol Metab 300: E211-220.

57. Clarke SF, Murphy EF, Nilaweera K, Ross PR, Shanahan F, et al. (2012) The gut microbiota and its relationship to diet and obesity: new insights. Gut Microbes 3: $186-202$.

58. Payne AN, Zihler A, Chassard C, Lacroix C (2012) Advances and perspectives in in vitro human gut fermentation modeling. Trends Biotechnol 30: 17-25.

59. Venema K, van den Abbeele P (2013) Experimental models of the gut microbiome. Best Pract Res Clin Gastroenterol 27: 115-126. 
60. Bussolo de Souza C, Roeselers G, Troost F, Jonkers D, Koenen ME, et al. (2014) Prebiotic effects of cassava bagasse in TNO's in vitro model of the colon in lean versus obese microbiota. J Funct Foods 11: 210-220.

61. Aguirre M, Jonkers DM, Troost FJ, Roeselers G, Venema K (2014) In vitro characterization of the impact of different substrates on metabolite production, energy extraction and composition of gut microbiota from lean and obese subjects. PLoS One 9: e113864.

62. Yang J, Keshavarzian A, Rose DJ (2013) Impact of dietary fiber fermentation from cereal grains on metabolite production by the fecal microbiota from normal weight and obese individuals. J Med Food 16: 862-867.

63. Sarbini SR, Kolida S, Gibson GR, Rastall RA (2013) In vitro fermentation of commercial alpha-gluco-oligosaccharide by faecal microbiota from lean and obese human subjects. Br J Nutr 109: 1980-1989.

64. Sarbini SR, Kolida S, Deaville ER, Gibson GR, Rastall RA (2014) Potential of novel dextran oligosaccharides as prebiotics for obesity management through in vitro experimentation. Br J Nutr 112: 1303-1314.

65. Condezo-Hoyos L, Mohanty IP, Noratto GD (2014) Assessing non-digestible compounds in apple cultivars and their potential as modulators of obese faecal microbiota in vitro. Food Chem 161: 208-215.

66. Aguirre M, Venema K (2015) The art of targeting gut microbiota for tackling human obesity. Genes Nutr 10: 472.

67. Allen-Vercoe E, Reid G, Viner N, Gloor GB, Hota S, et al. (2012) A Canadian Working Group report on fecal microbial therapy: microbial ecosystems therapeutics. Can J Gastroenterol 26: 457-462.

68. Petrof EO, Claud EC, Gloor GB, Allen-Vercoe E (2013) Microbial ecosystems therapeutics: a new paradigm in medicine? Benef Microbes 4: 53-65.

69. Petrof EO, Gloor GB, Vanner SJ, Weese SJ, Carter D, et al. (2013) Stool substitute transplant therapy for the eradication of Clostridium difficile infection: 'RePOOPulating' the gut. Microbiome 1: 3 .

70. Scott KP, Duncan SH, Flint HJ (2008) Dietary fibre and the gut microbiota. Nutrition Bulletin 33: 201-211.

71. Cani PD, Dewever C, Delzenne NM (2004) Inulin-type fructans modulate gastrointestinal peptides involved in appetite regulation (glucagon-like peptide-1 and ghrelin) in rats. Br J Nutr 92: 521-526.

72. Cani PD, Daubioul CA, Reusens B, Remacle C, Catillon G, et al. (2005) Involvement of endogenous glucagon-like peptide-1(7-36) amide on glycaemia-lowering effect of oligofructose in streptozotocin-treated rats. $J$ Endocrinol 185: 457-465.

73. Delzenne NM, Cani PD, Daubioul C, Neyrinck AM (2005) Impact of inulin and oligofructose on gastrointestinal peptides. Br J Nutr 93 Suppl 1: S157-161. 
74. Gibson GR, Roberfroid MB (1995) Dietary modulation of the human colonic microbiota: introducing the concept of prebiotics. J Nutr 125: 1401-1412.

75. Everard A, Lazarevic V, Derrien M, Girard M, Muccioli GG, et al. (2011) Responses of gut microbiota and glucose and lipid metabolism to prebiotics in genetic obese and diet-induced leptin-resistant mice. Diabetes 60: 2775-2786.

76. Frost G, Sleeth ML, Sahuri-Arisoylu M, Lizarbe B, Cerdan S, et al. (2014) The short-chain fatty acid acetate reduces appetite via a central homeostatic mechanism. Nat Commun 5: 3611.

77. Cani PD, Lecourt E, Dewulf EM, Sohet FM, Pachikian BD, et al. (2009) Gut microbiota fermentation of prebiotics increases satietogenic and incretin gut peptide production with consequences for appetite sensation and glucose response after a meal. Am J Clin Nutr 90: 1236-1243.

78. Cani PD, Joly E, Horsmans Y, Delzenne NM (2006) Oligofructose promotes satiety in healthy human: a pilot study. Eur J Clin Nutr 60: 567-572.

79. Cani PD, Possemiers S, Van de Wiele T, Guiot Y, Everard A, et al. (2009) Changes in gut microbiota control inflammation in obese mice through a mechanism involving GLP-2-driven improvement of gut permeability. Gut 58: 1091-1103.

80. Salazar N, Dewulf EM, Neyrinck AM, Bindels LB, Cani PD, et al. (2014) Inulintype fructans modulate intestinal Bifidobacterium species populations and decrease fecal short-chain fatty acids in obese women. Clin Nutr 34: 501-507.

81. Parnell JA, Reimer RA (2012) Prebiotic fibres dose-dependently increase satiety hormones and alter Bacteroidetes and Firmicutes in lean and obese JCR:LA-cp rats. Br J Nutr 107: 601-613.

82. Ursell LK, Van Treuren W, Metcalf JL, Pirrung M, Gewirtz A, et al. (2013) Replenishing our defensive microbes. BioEssays 35: 810-817.

83. Muegge BD, Kuczynski J, Knights D, Clemente JC, Gonzalez A, et al. (2011) Diet drives convergence in gut microbiome functions across mammalian phylogeny and within humans. Science 332: 970-974.

84. Wu GD, Chen J, Hoffmann C, Bittinger K, Chen YY, et al. (2011) Linking longterm dietary patterns with gut microbial enterotypes. Science 334: 105-108.

85. Salonen A, Lahti L, Salojarvi J, Holtrop G, Korpela K, et al. (2014) Impact of diet and individual variation on intestinal microbiota composition and fermentation products in obese men. ISME J 8: 2218-2230.

86. Korpela K, Flint HJ, Johnstone AM, Lappi J, Poutanen K, et al. (2014) Gut microbiota signatures predict host and microbiota responses to dietary interventions in obese individuals. PLoS One 9: e90702.

87. Gibson GR, Cummings JH, Macfarlane GT (1988) Use of a three-stage continuous culture system to study the effect of mucin on dissimilatory sulfate reduction and methanogenesis by mixed populations of human gut bacteria. Appl Environ Microbiol 54: 2750-2755. 
88. Venema K, Nuenen Mv, Smeets-Peeters M, Minekus M, Havenaar R (2000) TNO's in vitro large intestinal model: an excellent screening tool for functional food and pharmaceutical research. Ernährung 24: 558-564.

89. Verhoeckx K, Cotter P, López-Expósito I, Kleiveland C, Lea T, et al. (2015) The Impact of Food Bioactives on Health: Springer.

90. de Graaf AA, Maathuis A, de Waard P, Deutz NE, Dijkema C, et al. (2010) Profiling human gut bacterial metabolism and its kinetics using [U-13C] glucose and NMR. NMR in biomedicine 23: 2-12. 
Chapter 1 


\section{Chapter 2}

\section{To pool or not to pool? Impact of the use of individual and pooled fecal samples for in vitro fermentation studies}

Published as:

Aguirre M, Ramiro-Garcia J, Koenen ME, Venema K (2014) To pool or not to pool? Impact of the use of individual and pooled fecal samples for in vitro fermentation studies. J Microbiol Methods 107C: 1-7. 


\section{Abstract}

This study investigated the stability as well as the activity of the microbiota from a single and a pool of donors in the TNO in vitro model of the colon (TIM-2 system). Our findings underscore the substantial functional overlap in the microbiota of individuals when compared to a pool prepared from them, despite the differences observed in certain groups of bacteria detected during the fermentations. In all cases, high levels of acetate were produced followed by $n$-butyrate and propionate. The averaged cumulative amount of SCFA produced in the experiments was 206.3 $\pm 5.4 \mathrm{mmol}$ for the individual microbiotas while it was $210.3 \mathrm{mmol}$ for the pool. The sequencing analysis showed communities enriched in Firmicutes followed by Bacteroidetes, Actinobacteria, Proteobacteria and Verrucomicrobia.

These results show that the use of a pool of microbiota for in vitro studies does not result in a bacterial community with an aberrant profile and activity compared to that normally obtained from single donors. This demonstrates the suitability of the preparation of a pool of fecal samples to be used for fermentation experiments. 
To pool or not to pool?

\section{Introduction}

Monitoring the in vivo fermentation of carbohydrates in humans is difficult, due to the inaccessibility of the proximal colon, the ethical considerations and the medical supervision required. Validated in vitro models using human feces as an inoculum are much simpler systems that allow the study of the fermentation of any compound accompanied by the assessment of metabolites such as short-chain fatty acids (SCFA) and other bacterial products. Moreover, such systems constitute a powerful tool to follow disruptions in the microbial equilibrium in detail. Therefore, it is crucial to guarantee the development of a representative gut community in any of these systems without any confounding factor, unrelated with the aim of the study that would affect the composition of the microbiota.

TIM-2, the TNO in vitro model of the colon, is such an in vitro model that closely mimics the fermentation by the microbiota in the human large intestine allowing the growth of a highly complex, stable and dense $\left(\sim 10^{11} \mathrm{CFU} / \mathrm{mL}\right)$ active microbiota [1]. Studies performed in this system generally use a standardized inoculum which is derived from a pool of subjects of interest and is stored frozen. However, the use of an inoculum prepared from either a single donor or a pool of donors remains debatable among experts. The main argument relies in the concern about how representative such inoculum is in regard to the colonic ecosystem taking into account the abundance and the variety of bacterial species. Consequently, the use of an individual or a mixed inoculum is believed to lead to a degree of variation among experiments, even when the single inoculum is repeatedly taken from the same individual over time. Although it has been shown that the microbiota of adults is relatively stable over time, differences in the composition of the microbiota of a single individual over a 4 year period have been observed [2]. With respect to the TIM-2 system, no studies have been performed in order to find out differences related to the individual or pooling preparation of the inoculum in its composition and activity during a standard fermentation process with the use of a Standard Ileal Efflux Media (SIEM) as substrate. Still, previous data in this system using the individual inocula from 10 donors has shown that the microbial activity of the individual donors was extremely similar in functionality, despite a different microbiota composition $[3,4]$ corroborating the hypothesis that a standardized pool of gut microbiota can be used for these type of experiments.

It is also important to consider the report in which the effect of arabinoxylan and inulin has been tested using an inoculum prepared from a pooled donation (in TIM2) and from a single volunteer (in SHIME; Simulator of the Human Intestinal Microbial Ecosystem; another in vitro model) where a similar metabolic activity of the microbiota in both set ups was observed [5]. 
Thus, the purpose of this study was to compare both ways of preparing a fecal inoculum to be used in in vitro studies. Changes were identified by monitoring the composition and activity of the microbiota under standard fermentation experiments in TIM-2. To the best of our knowledge this is the first time that this direct comparison has been performed.

\section{Materials and Methods}

\section{Microbiota: source, collection and processing}

Individual fecal homogenates were prepared from a group of 4 healthy volunteers (age range 29-62; 3 males, 1 female). A homogenate with the pool of the microbiota from these individuals was also used. The group of participants was recruited at TNO (Zeist, The Netherlands). The individuals were non-smokers and had not used antibiotics, prebiotics, probiotics or laxatives 3 months prior to the donation. Informed consent was provided by each volunteer prior to the participation in the study.

Fresh fecal samples were directly collected in a tightly closed box with an anaerobic strip (AnaeroGen ${ }^{\mathrm{TM}}$, Oxoid, Cambridge, UK) inside. Within 1 hour after being collected, the donations were homogenized under strict anaerobic conditions in an anaerobic chamber (Bactron IV, Sheldon manufacturing, Cornelius, OR USA) containing $5 \% \mathrm{H}_{2}, 5 \% \mathrm{CO}_{2}$, and $90 \% \mathrm{~N}_{2} .8 .75 \mathrm{~g}$ of the fecal donation (individually as well as pooled) was mixed with a Turrax (IKA Ultra turrax T25 digital, Fisher Scientific Nederland) with a physiological saline preparation/dialysate (content per liter: $4.7 \mathrm{~g} \mathrm{~K}_{2} \mathrm{HPO}_{4} \cdot 3 \mathrm{H}_{2} \mathrm{O}, 8.4 \mathrm{~g} \mathrm{NaCl}, 0.009 \mathrm{~g}$ $\mathrm{FeSO}_{4} \cdot 7 \mathrm{H}_{2} \mathrm{O}, 0.8 \mathrm{~g} \mathrm{MgSO}_{4} \cdot 7 \mathrm{H}_{2} \mathrm{O}, 0.8 \mathrm{~g} \mathrm{CaCl}_{2} \cdot 2 \mathrm{H}_{2} \mathrm{O}, 0.7 \mathrm{~g}$ ox bile and $0.3 \mathrm{~g}$ cysteine hydrochloride) and glycerol (as cryo-protective agent).

\section{Feed used for fermentation: source and preparation}

The feeding substrate used for fermentation in TIM-2 simulates the average nondigestible carbohydrates consumed in a normal western diet [6]. SIEM is prepared with $0.5 \mathrm{~g}$ pectin, $0.5 \mathrm{~g}$ xylan, $0.5 \mathrm{~g}$ arabinogalactan, $0.5 \mathrm{~g}$ amylopectin and $4.5 \mathrm{~g}$ starch (Tritium Microbiology; Veldhoven, The Netherlands) per day. Specific details have been previously described $[7,8]$.

\section{TIM-2 Experimental protocol}

The TIM-2 system was flushed for $4 \mathrm{~h}$ with $\mathrm{N}_{2}$ prior to the introduction of the inoculum and it was maintained under this condition at $37{ }^{\circ} \mathrm{C}$ for $96 \mathrm{~h}$ with the $\mathrm{pH}$ kept at or above 5.8 by automatic titration with $2 \mathrm{M} \mathrm{NaOH}$. Water and fermentation products were removed from the lumen with a dialysate system (described in the 
following section) consisting of a semi-permeable hollow membrane which ran through the lumen. For all the experiments, the speed of the dialysis was set at 1.5 $\mathrm{ml} / \mathrm{min}$.

The feed preparation mentioned above was gradually introduced into the system in a total volume of $45 \mathrm{ml}$ in the adaptation period and $180 \mathrm{ml}$ over the $72 \mathrm{~h}$ of the test period at a rate of $2.5 \mathrm{ml} / \mathrm{h}$. Luminal content was maintained at a level of approximately $120 \mathrm{ml}$ in each unit by a level sensor (liquiphant FTL20-0025, Endress+Hauser).

After 24 and $48 \mathrm{~h}$ of fermentation $25 \mathrm{ml}$ of lumen sample was removed (Fig.1) through the system's sample port using a sterile syringe to mimic the transit of material from the proximal to the distal colon [7]. The dialysate fluid used in the system contained per liter: the dial preparation described under "Microbiota: source, collection and processing" section and $1 \mathrm{ml}$ of vitamin mixture containing per litre: $1 \mathrm{mg}$ menadione, $3 \mathrm{mg}$ D-biotin, $0.8 \mathrm{mg}$ vitamin B-12, $15 \mathrm{mg}$ pantothenate, $7 \mathrm{mg}$ nicotinamide, $7 \mathrm{mg}$ para-aminobenzoic acid, and $6 \mathrm{mg}$ thiamine (all from Tritium Microbiology).

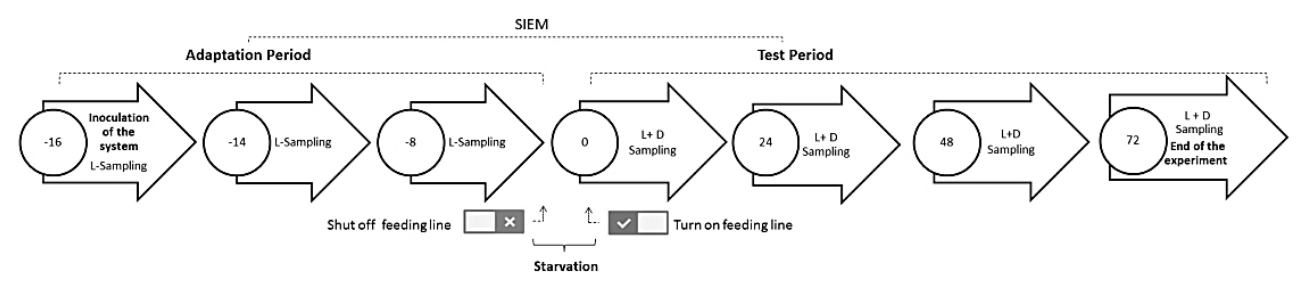

Fig. 1. Experimental set up (not at scale). L-Sampling = luminal samples $;$ D-Sampling = Dialysate.

\section{Design of the study}

Approximately $30 \mathrm{ml}$ portions of fecal homogenate $( \pm 25 \% \mathrm{w} / \mathrm{v})$ were used to inoculate the separate TIM-2 units for each experiment. Each unit was then filled to $120 \mathrm{ml}$ with dialysate (dial). Right after the inoculation, the microbiota was left to adapt $(16 \mathrm{~h})$ to the new environment. After this adaptation period, the culture was deprived from SIEM for $2 \mathrm{~h}$ which aims at full use of fermentable carbohydrates prior to feeding of a test carbohydrate. Minekus et al. [9] explained that this starvation period was established when the lack of production of acids was observed in the system when the feeding line was turned off.

Next, units were fed with SIEM until the end of the experiment. Samples were taken from the lumen after -16, -14, -8, 0, 24, 48 and $72 \mathrm{~h}$. Dial was collected after 0, 24, 48 and $72 \mathrm{~h}$ (Fig.1). Both luminal and dial samples were first snap frozen in liquid nitrogen $\left(-196{ }^{\circ} \mathrm{C}\right)$ immediately after collection and were stored at $-80{ }^{\circ} \mathrm{C}$ previous to analysis. 


\section{Analytical methods}

With the samples from lumen and dialysate the production of SCFA and branchedchain fatty acids (BCFA) were calculated. Microbial composition was sequenced at $\mathrm{t}_{-16}$ and $\mathrm{t}_{72}$.

SCFA (acetate, propionate, and $n$-butyrate) and BCFA (iso-butyrate and isovalerate)

Samples for SCFA and BCFA analyses were determined by analyzing their concentration by GC (Stabilwax-DA, length $15 \mathrm{~m}$, ID $0.53 \mathrm{~mm}$, film thickness 0.1 mm; Varian Chrompack, Bergen op Zoom, the Netherlands). Samples were prepared as previously described by van Nuenen et al. [8].

Phylogenetic analysis of the microbiota

DNA from the luminal samples was isolated using the AGOWA mag Mini kit (DNA Isolation Kit, AGOWA, Berlin, Germany), according to the manufacturer's instructions.

The generation of the PCR amplicon library was performed by amplification of V5-V7 hypervariable region of the small subunit ribosomal DNA gene (16S rDNA). Amplification was performed using the forward primer $785 \mathrm{~F}$ (5'GGATTAGATACCCBRGTAGTC-3') and reverse primer 1175R ('5ACGTCRTCCCCDCCTTCCTC-3). The primers were fitted with the Roche 454 (Branford, CT, USA) Adapter A (forward primer) and B (reverse primer), fused to the 5' end of the 16S rDNA bacterial primer sequences. The forward primer also included a unique nucleotide barcode. The amplification mix contained 2 units of PfuUltra II Fusion HS DNA polymerase (Stratagene, La Jolla, CA, USA) and 1x PfuUltra II reaction buffer (Stratagene), $200 \mu \mathrm{M}$ dNTP PurePeak DNA polymerase Mix (Pierce Nucleic Acid Technologies, Milwaukee, WI, USA), and $0.2 \mu \mathrm{M}$ of each primer.

After an initial denaturation $\left(94^{\circ} \mathrm{C} ; 2 \mathrm{~min}\right), 30$ cycles were performed that consisted of denaturation $\left(94^{\circ} \mathrm{C} ; 30 \mathrm{sec}\right)$, annealing $\left(50^{\circ} \mathrm{C} ; 40 \mathrm{~s}\right)$, and extension $\left(72{ }^{\circ} \mathrm{C} ; 80 \mathrm{~s}\right.$ ). Samples with DNA recovery of equal or less than $10 \mathrm{pg} / \mu 1$ of DNA were cycled 35 times using the same protocol.

Amplicons were size checked and quantified by gel electrophoresis and Quant-iT Picogreen dsDNA Assay (Invitrogen, Carlsbad, CA, USA) on the Tecan Infinite M200 (Tecan Group Ltd, Männedorf, Switzerland). Amplicons of the individual samples were equimolar pooled and purified from agarose gel after electrophoresis by means of QIAquick Gel Extraction Kit Protocol (Qiagen, Hilden, Germany).

The library was sequenced unidirectionally in the forward direction (A-adaptor) in one run in the 454 GS-flx-Titanium Sequencer (Roche) by Keygene (Wageningen, The Netherlands). 
FASTA-formatted sequences and corresponding quality scores were extracted from the .sff data file generated by the GS-FLX-Titanium sequencer using the GS Amplicon software package (Roche).

All data extraction, pre-processing, analysis of operational taxonomic units (OTUs), and classifications were performed using modules implemented in the Mothur v. 1.22.2. software platform [10] as in Roeselers et al. [11].

In brief, unique barcodes were used to sort sequences by sample of origin. Subsequently, barcodes, primer sequences and low quality data (containing ambiguous base calls $(\mathrm{N})$ in the sequence, more than 8 homopolymers anywhere in the sequence, shorter than $50 \mathrm{nt}$ after trimming with a window average below 35 or a length $>500$ or $<200 \mathrm{bp}$ ) were removed. The data set was simplified by using the "unique.seqs" command to generate a non-redundant (unique) set of sequences. Sequences were 'denoised' using the "pre.cluster" command [12]. Unique sequences were aligned using the "align.seqs" command and an adaptation of the Bacterial SILVA SEED database as a template [13]. In order to ensure that comparable regions of the 16S rDNA gene were analyzed across all reads, sequences that started before the 2.5-percentile or ended after the 97.5-percentile in the alignment were filtered.

A total of 16814 potentially chimeric sequences were detected and removed using the "chimera.uchime" command [14]. High quality aligned sequences were classified by using the RDP-II naïve Bayesian Classifier [15] using a 60\% confidence threshold. Aligned sequences were also clustered into operational taxonomic units (OTUs; defined by $97 \%$ similarity) and were calculated by the average linkage clustering method. Unclassified sequences were also grouped in OTUs and were represented with a random number to distinguish them from other unclassified OTUs found within the same phyla. A matrix table that reports the taxonomic assignment and the frequency obtained for each OTU was used to generate a biom table file which was subsequently employed for diversity analysis using Quantitative Insights Into Microbial Ecology (QIIME) software [16] . Rarefaction curves were computed with the "alpha_rarefaction.py" QIIME script, using Simpson metric and a rarefaction depth value of 5000 sequences. Principal Coordinate Analysis (PCoA) plots were obtained using the "beta_diversity_through_plots.py" command, selecting Weighted UniFrac as desired metric to generate the distance matrix. Community profiles were compared using this metric [17].

\section{Data presentation}

The experiments were performed in series of one $(n=1)$ per type of inoculum (i.e., individual or pool). To avoid unnecessary repetition, this is not indicated further in 
the text or graphs in the Results section. Because of this, no statistical analysis could be executed. We have shown before that the TIM-2 system is very reproducible.

\section{Results}

\section{Microbiota activity}

\section{SCFA}

There was a substantial functional overlap in the microbiota of the four different individuals and the pool (Fig.2a). The averaged cumulative amount of SCFA produced in the TIM-2 was $206.3 \pm 5.4 \mathrm{mmol}$ (Fig. S1a and b) for the individuals while it was $210.3 \mathrm{mmol}$ for the pool. Especially the daily cumulative production of SCFA for individual (Ind.) 1, 4 and the pool was similar. In all the fermentations acetate was highly produced followed by butyrate and propionate (Fig. 3).

\section{BCFA}

In the case of BCFA production, more inter-individual variation was observed. The production obtained with the inocula prepared from the Ind. 1 and 4 was lower when compared to that from Ind. 2 and 3 (Fig.2b). Still, similar kinetics for BCFA production could be observed between Ind. 2, 3 and the pool (Fig. 4). The averaged cumulative amount of BCFA produced in the TIM-2 was $5.9 \pm 2.2$ mmol (Fig. S1c and d) for the individuals while it was $7.3 \mathrm{mmol}$ for the pool.
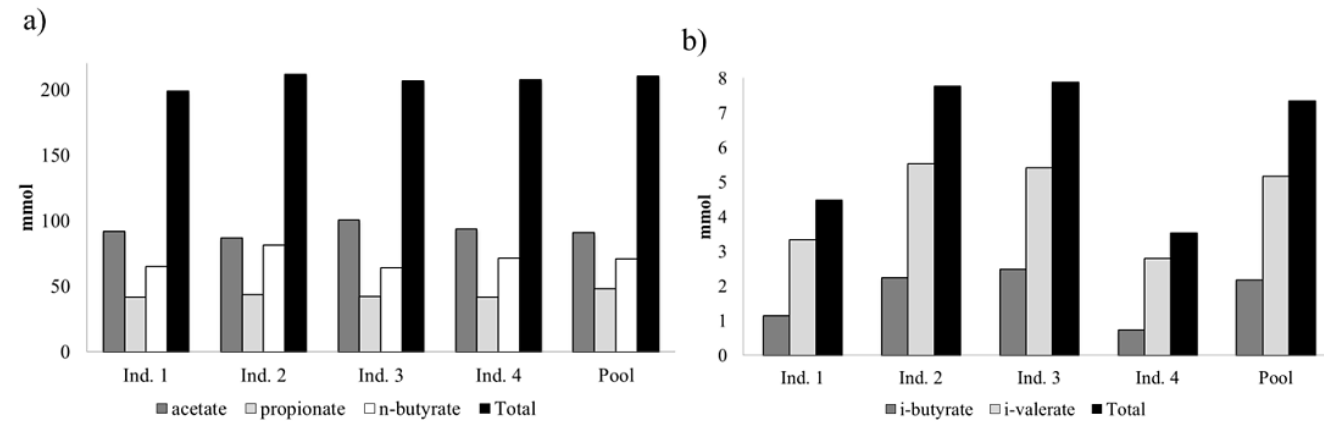

Fig 2. a) Final SCFA production ( $\left.t_{72}\right)$ for all the fermentations; b) Final BCFA production $\left(\mathrm{t}_{72}\right)$ for all the fermentations.

\section{Microbiota composition}

The ratio between a sampling time point and $t_{-16}$ was calculated (e.g., $\left.t_{72} / t_{-16}\right)$. The ratio for this value and the pool was then determined to obtain fold changes. A 
value equal to 1 indicates no change; a value of $>1$ indicates an increase; and a value of $<1$ indicates a decrease of the respective microbial genera (Fig. 5; Table1).

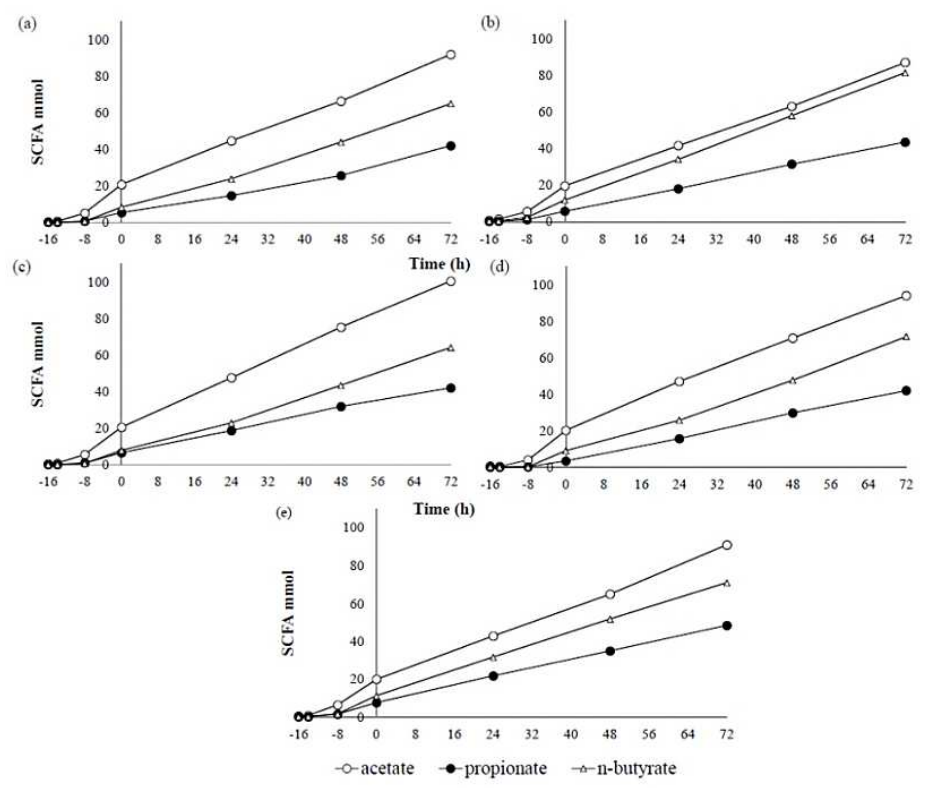

Fig. 3. Cumulative production of SCFA in (a) Ind. 1, (b) Ind. 2, (c) Ind. 3, (d) Ind. 4 and (e) pool during $72 \mathrm{~h}$ fermentation of SIEM.

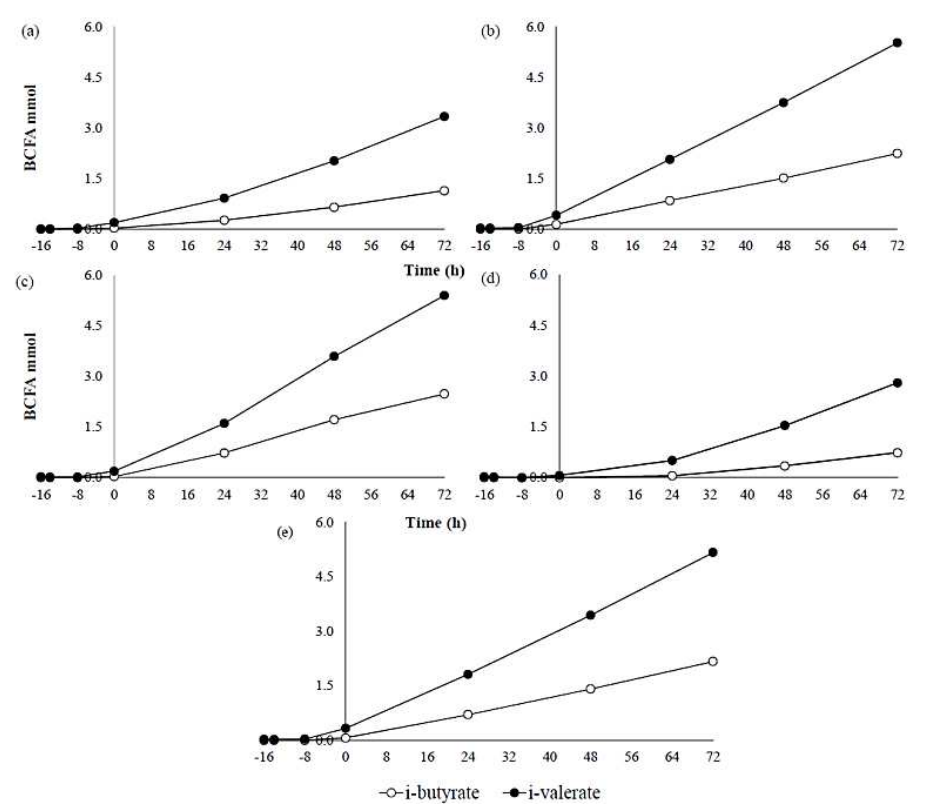

Fig. 4. Cumulative production of BCFA in (a) Ind. 1, (b) Ind. 2, (c) Ind. 3, (d) Ind. 4 and (e) pool during $72 \mathrm{~h}$ fermentation experiments. 


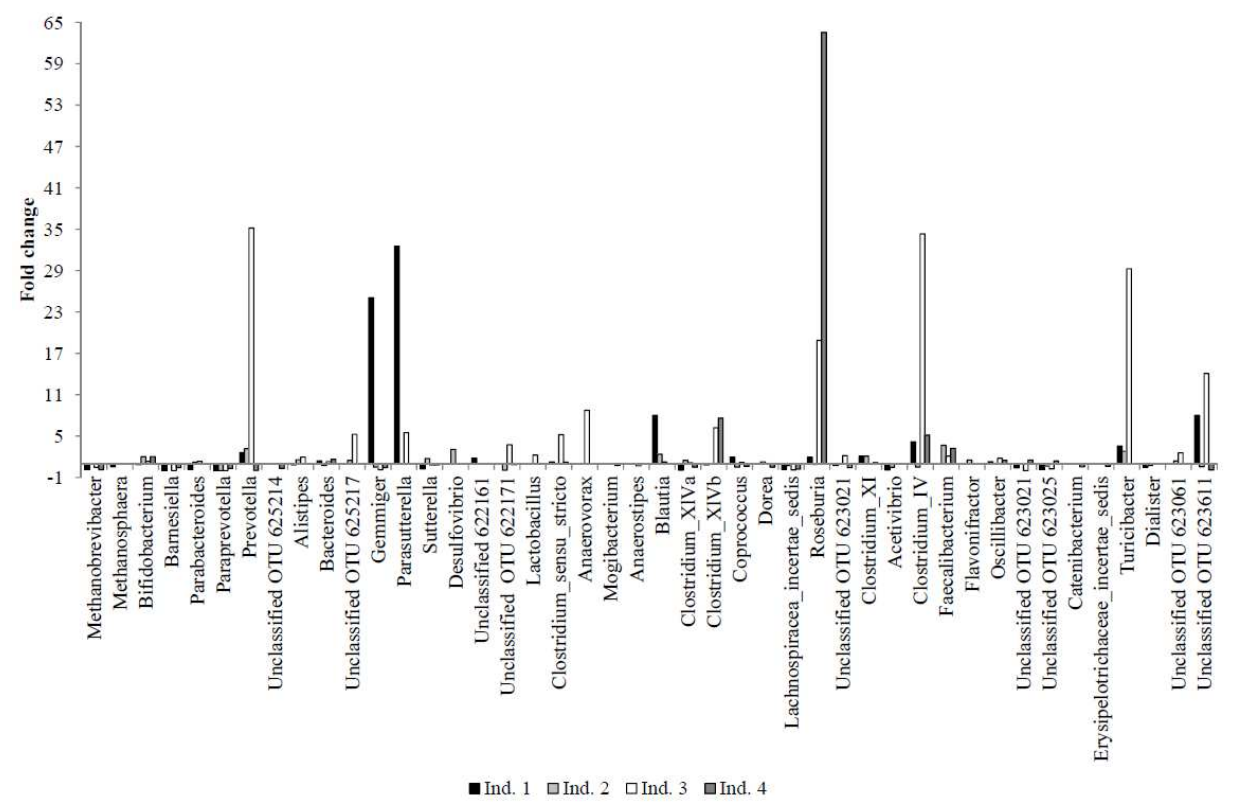

Fig. 5. Fold change in the microbiota from the different inocula at the genus level.

At the genus level it could be observed that many bacterial groups from the individuals remained similar in abundance to the pool (fold change between 1 and 1). Some groups presented higher levels in some individuals (e.g., Prevotella, Roseburia, Clostridium IV and Turicibacter in Ind. 3; Table 1). 
Table 1. Fold change in the individuals with respect to the pool at the genus level ${ }^{\mathrm{a}}$.

\begin{tabular}{|c|c|c|c|c|}
\hline \multirow[b]{2}{*}{ Genus } & \multicolumn{4}{|c|}{ Fold change with respect to the pool } \\
\hline & Ind. 1 & Ind. 2 & Ind. 3 & Ind. 4 \\
\hline Methanobrevibacter & 0.2 & & 0.5 & 0.1 \\
\hline Methanosphaera & 0.5 & & & \\
\hline Bifidobacterium & 0.8 & 2.0 & 1.3 & 2.0 \\
\hline Barnesiella & & & & 0.4 \\
\hline Parabacteroides & 0.1 & 1.2 & 1.3 & 0.9 \\
\hline Paraprevotella & & & & 0.3 \\
\hline Prevotella & 2.6 & 3.1 & 35.2 & \\
\hline Unclassified OTU 625214 & & & & 0.3 \\
\hline Alistipes & 0.8 & 1.5 & 1.9 & 1.0 \\
\hline Bacteroides & 1.4 & 0.7 & 1.3 & 1.6 \\
\hline Unclassified OTU 625217 & & 1.5 & 5.3 & \\
\hline Gemmiger & 25.1 & 0.5 & 0.1 & 0.4 \\
\hline Parasutterella & 32.5 & & 5.5 & \\
\hline Sutterella & 0.2 & 1.7 & 0.8 & 0.8 \\
\hline Desulfovibrio & & 3.1 & & \\
\hline Unclassified OTU 22161 & 1.8 & 0.9 & & \\
\hline Unclassified OTU 622171 & & & 3.7 & 0.9 \\
\hline Lactobacillus & & & 2.3 & \\
\hline Clostridium_sensu_stricto & 1.2 & 1.0 & 5.2 & 1.2 \\
\hline Anaerovorax & & & 8.7 & \\
\hline Mogibacterium & & & & 0.7 \\
\hline Anaerostipes & & & 0.7 & \\
\hline Blautia & 8.0 & 2.4 & 1.2 & 0.9 \\
\hline Clostridium_XlVa & 0.1 & 1.5 & 1.1 & 0.5 \\
\hline Clostridium_XlVb & 0.8 & & 6.2 & 7.6 \\
\hline Coprococcus & 1.9 & 0.5 & 1.2 & 0.6 \\
\hline Dorea & 0.9 & 1.3 & 0.9 & 0.5 \\
\hline Lachnospiraceae incertae sedis & 0.2 & 0.7 & 0.1 & 0.2 \\
\hline Roseburia & 2.0 & 0.9 & 18.9 & 63.5 \\
\hline Unclassified OTU 623021 & 0.7 & 1.0 & 2.1 & 0.4 \\
\hline Clostridium_XI & 2.1 & 2.1 & & 1.1 \\
\hline Acetivibrio & 0.1 & 0.5 & & \\
\hline Clostridium_IV & 4.2 & 0.5 & 34.3 & 5.1 \\
\hline Faecalibacterium & 0.9 & 3.7 & 2.1 & 3.2 \\
\hline Flavonifractor & & 1.5 & & \\
\hline Oscillibacter & 1.3 & 0.9 & 1.8 & 1.5 \\
\hline Unclassified OTU 623021 & 0.4 & 0.9 & & 1.5 \\
\hline
\end{tabular}


Chapter 2

$\begin{array}{lcccc}\text { Unclassified OTU 623025 } & 0.2 & 0.6 & 0.2 & 1.4 \\ \text { Catenibacterium } & & & & 0.5 \\ \text { Erysipelotrichaceae_incertae_sedis } & & & & 0.6 \\ \begin{array}{l}\text { Turicibacter } \\ \text { Dialister }\end{array} & 3.6 & 2.8 & 29.3 & \\ \text { Unclassified OTU 623061 } & 0.5 & 0.7 & & \\ \text { Unclassified OTU 623611 } & & 1.4 & 2.6 & \\ & 8.0 & 0.6 & 14.1 & 0.1\end{array}$

${ }^{\mathrm{a}}$ For each fermentation the ratio between a sampling time point and $\mathrm{t}$ $=-16$ was calculated (e.g., $\mathrm{t}_{72} / \mathrm{t}_{-16}$ ). The ratio for this value and the pool was then determined to obtain fold changes. A value equal to 1 indicates no change; a value of $>1$ indicates an increase; and a value of $<1$ indicates a decrease of the respective microbial genera.

PCoA plots (Fig. S2) show that the different individuals clustered together to the pool at both times $\left(\mathrm{t}_{-16}\right.$ and $\left.\mathrm{t}_{72}\right)$. In addition, ranking the diversity in the individuals versus the pool using the refraction curve (Simpson metric) demonstrated that they were similar among each other (Fig. S3).

\section{Discussion}

The characterization of the microbiota composition in well-established in vitro systems has been possible using high phylogenetic resolution. These analyses confirm the use of in vitro systems as effective tools to study the influence of medication and food components on the composition and activity of gut bacteria $[18,19]$. Nevertheless, limitations such as variations in carbohydrate content of the feeding media, lack of host interactions, variations in oxygen levels, among others, may result in discrepancies between studies [20].

Despite this, it has been observed that models such as TIM-2 can effectively maintain a stable and highly reproducible gut microbiota similar to the donors from whom it was prepared, as well as mimicking its microbial functionality [6,21]. Importantly, the design of the studies in TIM-2 includes a control setup which is operated in parallel with the test experiment in order to ensure that the changes observed originate from the treatment itself.

Experiments involving in vitro fermentations have helped to simulate an ample number of different conditions such as age, diseases and disorders [18]. One challenge has been the lack of literature information related with the ability of in vitro systems in developing representative and reproducible bacterial communities from fecal samples of a single individual or a pool of individuals [20]. It is presumed by many that any of these two variations may account for significant 
changes in the functional aspect and general diversity from the inoculum, although we have shown before that there is an enormous overlap in functionality within fecal samples from different $(n=10)$ individuals [4]. The use of pooled microbiota as an inoculum for in vitro fermentation studies brings important advantages: i) a standardized microbiota can be used for multiple (up to 100) different experiments, and ii) it is presumed to have a more diverse population of bacteria and thus the use of a more representative microbiota for the whole population,

The present study demonstrates a similar metabolic activity in terms of SCFA production in experiments using inocula prepared from individuals and a pool of these. The fact that the cumulative SCFA production was similar (Fig. 2) provides a good indication that the metabolic response of the microbiota present in both types of inoculum was the same under both conditions. Nevertheless, small differences in fermentation kinetics occurred (Fig. 3), which indicates that such variation could be attributable to the composition of the community present in the collected feces.

In all cases, high levels of acetate were reported followed by $n$-butyrate and propionate. It is suggested that the similar concentrations of SCFA detected in all experiments are the result of a unique induction and utilization of specific metabolic pathways proper for the substrates provided to the inocula. Indeed, the integration of metabolite and molecular data has suggested that a metabolic crossfeeding occurs in in vitro systems such as TIM-2 [22]. During cross-feeding, several microbes contribute in the production of the metabolites [6]. Therefore, certain metabolites could be used as substrate for the metabolism of another [23]. For instance, one of the major pathways to butyrate production is via the conversion of acetate to butyrate which is catalyzed by butyryl coA-acetate coA transferase [24].

It was observed that the main BCFA produced was iso-valerate followed by isobutyrate (Fig. 4). This was expected since it has been found that the population of bacteria capable of producing BCFA as major end products may range from $0.6 \%$ (iso-valerate) to $40 \%$ (iso-butyrate) in healthy subjects [25].

The different cumulative amounts observed in BCFA production (Fig. 2b) could be explained due to the fact that fresh inocula (inoculum immediately used after its collection) was used in the present study. Fresh inocula still contains an important part of unfermented proteinaceous components from the host which makes it metabolically active. It has been observed that trichloroacetic acid-soluble peptides and proteins are present in high concentrations in the intestine and feces of humans [25]. Furthermore, amino acid pools have been also detected in proximal and distal contents which are highly influenced by host diet and the release of desquamated epithelial and bacterial intracellular contents, colonic and pancreatic secretions and 
intestinal absorption [25]. Since BCFA are only produced in small amounts compared to SCFA, a larger inter-individual variation is not unexpected. This interindividual variation, together with the similar production of SCFA, argues in favor of a pool of fecal samples to be used in in vitro studies.

Individual fecal inocula from each experiment were compared against the pool inoculum before and after performing the experiments to examine how the community composition changed in the in vitro study. In both sampling times (i.e., $\mathrm{t}_{-16}$ and $\mathrm{t}_{72}$ ), the sequencing analysis of all the experiment runs showed communities enriched in Firmicutes followed by Bacteroidetes, Actinobacteria, Proteobacteria and Verrucomicrobia (Fig. S4). The results also demonstrate high individual specificity in the different inocula at the genus level (Table 1) which was expected based on previous studies [26,27]. Despite this, the majority of OTU's are shared among the pooled inoculum and the individuals. Members of the genera Oscillibacter, Alistipes, Bacteroides and Dorea in the individual inocula were detected at similar levels when compared to the pooled inoculum (1.2 average fold change; Fig. 5). Data from studies analyzing the composition of gut bacteria in healthy subjects indicate that, at least in some participants, members of the last three genera mentioned above prevail and constitute a significant part of their bacterial consortium [26,28].

Prevotella, Gemmiger, Parasutterella, Roseburia, Clostridium cluster IV and Turicibacter members were also found to be prevalent in some of the individual libraries and the pool. In the present study, a considerable abundance of Prevotella, Clostridium cluster $I V$ and Turicibacter was detected in Ind.3 compared to the pooled inocula ( 29 to 35 fold change). Such difference is suggested to come from the diversity naturally expected within the host's bacterial community of individuals, influenced by many factors, including diet. Increasing evidence shows that diet influences the composition of the microbiota. Perhaps one of the most important clinical studies performed in humans in order to elucidate the interaction between diet and microbiota shows that short-term diets do not have any influence on the gut bacterial composition while long-term diets do [29]. The composition of gut microbiota in subjects following a diet rich in animal fat, protein and saturated fats differs from subjects with a diet low in these components but rich in carbohydrates and simple sugars [30].

Overall, despite the pronounced differences observed in certain groups of bacteria detected during the (individual) fermentations our analyses highlight that there is no big difference in terms of diversity (Fig. S3). We found that in a pooled sample there is a variation which determines how rich the bacterial community is; such variation is slightly larger than a normal individual but it is more stochastic (Fig. S2). Furthermore, our findings underscore the substantial functional overlap in the 
microbiota of individuals when compared to a pool prepared from these, and argue in favor of using a pool when comparing different experimental conditions, such as changes in carbohydrate substrate, or addition of more protein or fat. The next step to proceed is to determine the number of donors which could be used in the preparation. This makes it feasible in finding a representative inoculum to perform a wide range of in vitro studies for food and pharmaceutical studies.

\section{Conclusion}

The assessment of specific microbiota effects can be performed with several donors tested in separate fermentation systems with the limitation of the use of significant monetary and human resources [31]. The relevance of the current findings is that the use of a pool of microbiota for in vitro studies does not result in a bacterial community with an aberrant profile and activity compared to that normally obtained from single donors. This demonstrates the suitability of the preparation of a pool of fecal sample to be used for fermentation experiments.

\section{Acknowledgments}

The authors thank Jordy Coolen and Martien Caspers for their instructions and guidance during the sequencing analysis; Rob Havenaar and Wendy Borst for their technical assistance with sampling, and Peter Schaap and Erwin Zoetendal for their valuable input during the discussion of the diversity analysis. This study was partly funded by the Top Institute Food \& Nutrition (GH004) (TIFN, Wageningen, The Netherlands). 


\section{References}

1. De Graaf AA, Maathuis A, De Waard P, Deutz NEP, Dijkema C, et al. (2010) Profiling human gut bacterial metabolism and its kinetics using [U-13C] glucose and NMR NMR in Biomedicine 23: 2-12.

2. Rajilic-Stojanovic M, Heilig HG, Tims S, Zoetendal EG, de Vos WM (2012) Longterm monitoring of the human intestinal microbiota composition. Environ Microbiol 15: 1146-1159.

3. Venema K (2012) Intestinal fermentation of lactose and prebiotic lactose derivatives, including human milk oligosaccharides. Int Dairy J 22: 123-140.

4. Venema K, van Nuenen MHMC, Heuvel E, G. van den, Pool W, van der Vossen JMBM (2003) The Effect of Lactulose on the Composition of the Intestinal Microbiota and Short-chain Fatty Acid Production in Human Volunteers and a Computer-controlled Model of the Proximal Large Intestine. Microb Ecol Health Dis 15: 94-105.

5. Van den Abbeele P, Venema K, Van de Wiele T, Verstraete W, Possemiers S (2013) Different Human Gut Models Reveal the Distinct Fermentation Patterns of Arabinoxylan versus Inulin. J Agric Food Chem 61: 9819-9827.

6. Maathuis AJH, van den Heuvel EG, Schoterman MHC, Venema K (2012) GalactoOligosaccharides Have Prebiotic Activity in a Dynamic in vitro Colon Model Using a C-Labeling Technique. J Nutr 142: 1205-1212.

7. Maathuis A, Hoffman A, Evans A, Sanders L, Venema K (2009) The Effect of the Undigested Fraction of Maize Products on the Activity and Composition of the Microbiota Determined in a Dynamic in Vitro Model of the Human Proximal Large Intestine. J Am Coll Nutr 28: 657-666.

8. Van Nuenen HMC, Meyer PD, Venema K (2003) The effect of various inulins and Clostridium difficile on the metabolic activity of the human colonic microbiota in vitro. Microb Ecol Health Dis 15: 137-144.

9. Minekus M, Smeets-Peeters M, Bernalier A, Marol-Bonnin S, Havenaar R, et al. (1999) A computer-controlled system to simulate conditions of the large intestine with peristaltic mixing, water absorption and absorption of fermentation products. Appl Microbiol Biotechnol 53: 108-114.

10. Schloss PD, Westcott SL, Ryabin T, Hall JR, Hartmann M, et al. (2009) Introducing mothur: open-source, platform-independent, community-supported software for describing and comparing microbial communities. Appl Environ Microbiol 75: 7537-7541.

11. Roeselers G, Mittge EK, Stephens WZ, Parichy DM, Cavanaugh CM, et al. (2011) Evidence for a core gut microbiota in the zebrafish. Isme J 5: 1595-1608.

12. Huse SM, Welch DM, Morrison HG, Sogin ML (2010) Ironing out the wrinkles in the rare biosphere through improved OTU clustering. Environ Microbiol 12: 1889-1898. 
13. Pruesse E, Quast C, Knittel K, Fuchs BM, Ludwig W, et al. (2007) SILVA: a comprehensive online resource for quality checked and aligned ribosomal RNA sequence data compatible with ARB. NAR 35: 7188-7196.

14. Edgar RC, Haas BJ, Clemente JC, Quince C, Knight R (2011) UCHIME improves sensitivity and speed of chimera detection. Bioinformatics 27: 2194-2200.

15. Wang Q, Garrity GM, Tiedje JM, Cole JR (2007) Naive Bayesian Classifier for Rapid Assignment of rRNA Sequences into the New Bacterial Taxonomy. Appl Environ Microbiol 73: 5261-5267.

16. Caporaso JG, Kuczynski J, Stombaugh J, Bittinger K, Bushman FD, et al. (2010) QIIME allows analysis of high-throughput community sequencing data. Nat Methods 7: 335-336.

17. Hamady M, Lozupone C, Knight R (2010) Fast UniFrac: facilitating highthroughput phylogenetic analyses of microbial communities including analysis of pyrosequencing and PhyloChip data. ISME J 4: 17-27.

18. Venema K, van den Abbeele P (2013) Experimental models of the gut microbiome. Best Pract Res Clin Gastroenterol 27: 115-126.

19. Roeselers G, Bouwman J, Venema K, Montijn R (2012) The human gastrointestinal microbiota -an unexplored frontier for pharmaceutical discovery. Pharmacol Res 66: 443-447.

20. McDonald JA, Schroeter K, Fuentes S, Heikamp-Dejong I, Khursigara CM, et al. (2013) Evaluation of microbial community reproducibility, stability and composition in a human distal gut chemostat model. J Microbiol Methods 95: 167-174.

21. Rajilic-Stojanovic M, Maathuis A, Heilig H, Venema K, De Vos W, et al. (2010) Evaluating the microbial diversity of an in vitro model of the human large intestine by phylogenetic microarray analysis. Microbiology 156: 3270-3281.

22. Kovatcheva-Datchary P, Egert M, Maathuis A, Rajilic-Stojanovic M, De Graaf AA, et al. (2009) Linking phylogenetic identities of bacteria to starch fermentation in an in vitro model of the large intestine by RNA-based stable isotope probing. Environ Microbiol 11: 914-992.

23. Wintermute EH, Silver PA (2010) Dynamics in the mixed microbial concourse. Genes Dev 24: 2603-2614.

24. Payne AN, Chassard C, Zimmermann M, Muller P, Stinca S, et al. (2011) The metabolic activity of gut microbiota in obese children is increased compared with normal-weight children and exhibits more exhaustive substrate utilization. Nutr Diabetes 1: e12.

25. Smith EA, Macfarlane GT (1998) Enumeration of amino acid fermenting bacteria in the human large intestine: effects of $\mathrm{pH}$ and starch on peptide metabolism and dissimilation of amino acids. FEMS Microbiol Ecol 25: 355-368.

26. Tap J, Mondot S, Levenez F, Pelletier E, Caron C, et al. (2009) Towards the human intestinal microbiota phylogenetic core. Environ Microbiol 11: 2574-2584. 
27. Woodmansey E, J.,, McMurdo MET, Macfarlane GT, Macfarlane S (2004) Comparison of Compositions and Metabolic Activities of Fecal Microbiotas in Young Adults and in Antibiotic-Treated and Non-Antibiotic-Treated Elderly Subjects. Appl Environ Microbiol 70: 6113-6122.

28. Kulagina EV, Efimov BA, Maximov PY, Kafarskaia LI, Chaplin AV, et al. (2012) Species composition of Bacteroidales order bacteria in the feces of healthy people of various ages. Biosci Biotechnol Biochem 76: 169-171.

29. Moschen AR, Wieser V, Tilg H (2012) Dietary Factors: Major Regulators of the Gut's Microbiota. Gut Liver 6: 411-416.

30. Wu GD, Chen J, Hoffmann C, Bittinger K, Chen Y-Y, et al. (2011) Linking LongTerm Dietary Patterns with Gut Microbial Enterotypes. Science 334: 105-108.

31. Payne AN, Zihler A, Chassard C, Lacroix C (2012) Advances and perspectives in in vitro human gut fermentation modeling. Trends Biotechnol 30: 17-25. 


\section{Supplemental material}

(a)

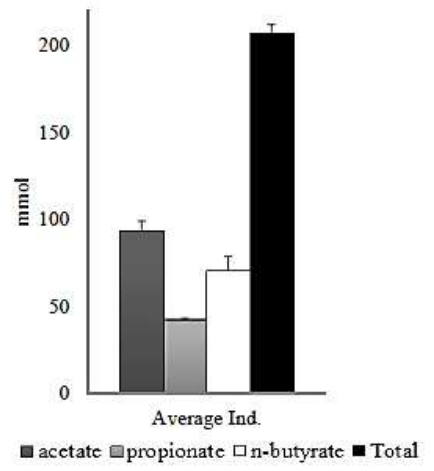

(c)

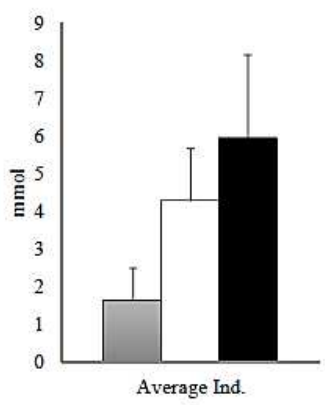

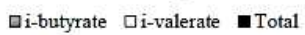
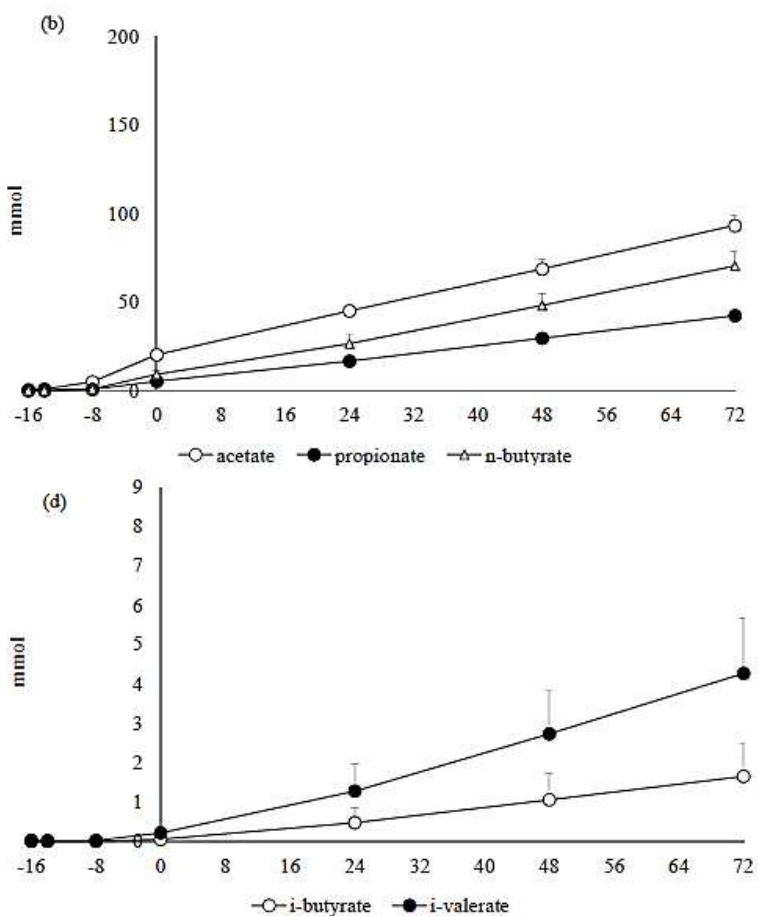

Fig. S1. Average metabolites produced in the experiments with the individuals $(\mathrm{n}=4)$. (a) Final SCFA production $\left(\mathrm{t}_{72}\right)$, (b) cumulative production of SCFA and (c) final BCFA production $\left(\mathrm{t}_{72}\right)$ and (d) cumulative production of BCFA.

a)

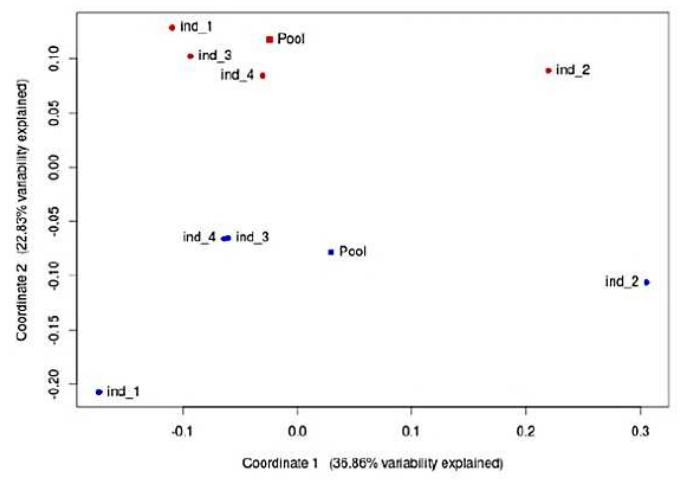

b)

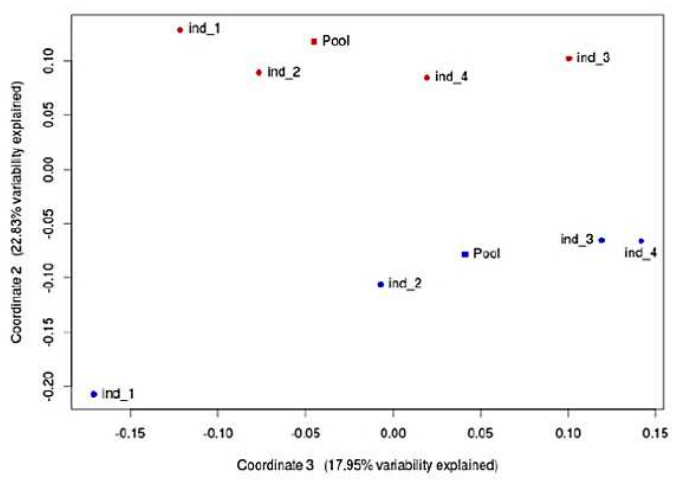

Fig. S2. Principal Coordinates Analysis using Weighted UniFrac distances a) coordinate 1 and 2 together explain $59.69 \%$ of the 
variability, b) coordinate 2 and 3 together explain $40.78 \%$ of the variability. Pool samples are represented by squares while individual samples are represented by circles. Samples at time -16 hours are depicted in red and samples at time 72 hours are depicted in blue.

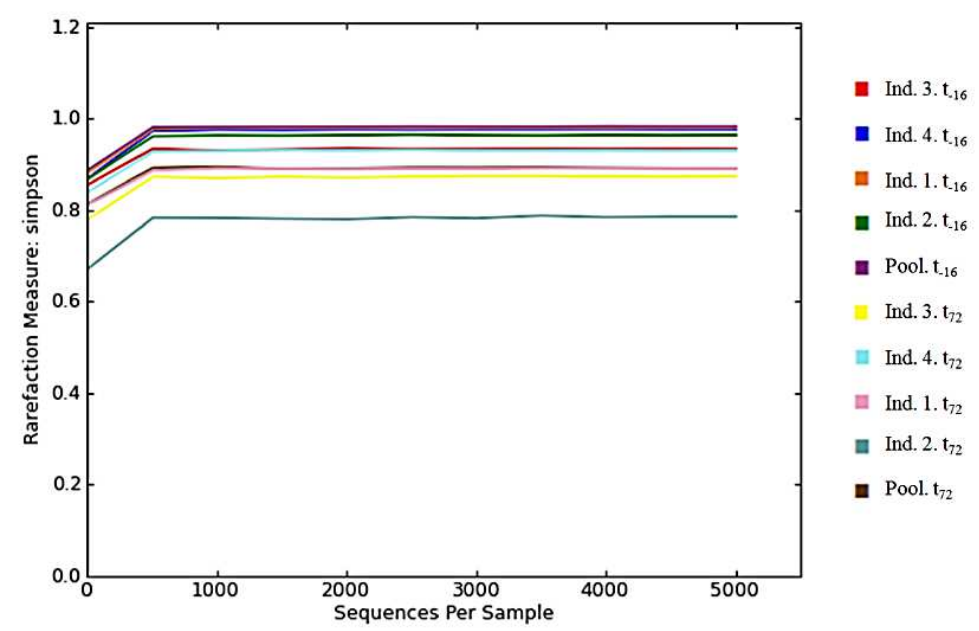

Fig. S3. Rarefaction curves were made using Simpson metric and a rarefaction depth value of 5000 sequences.

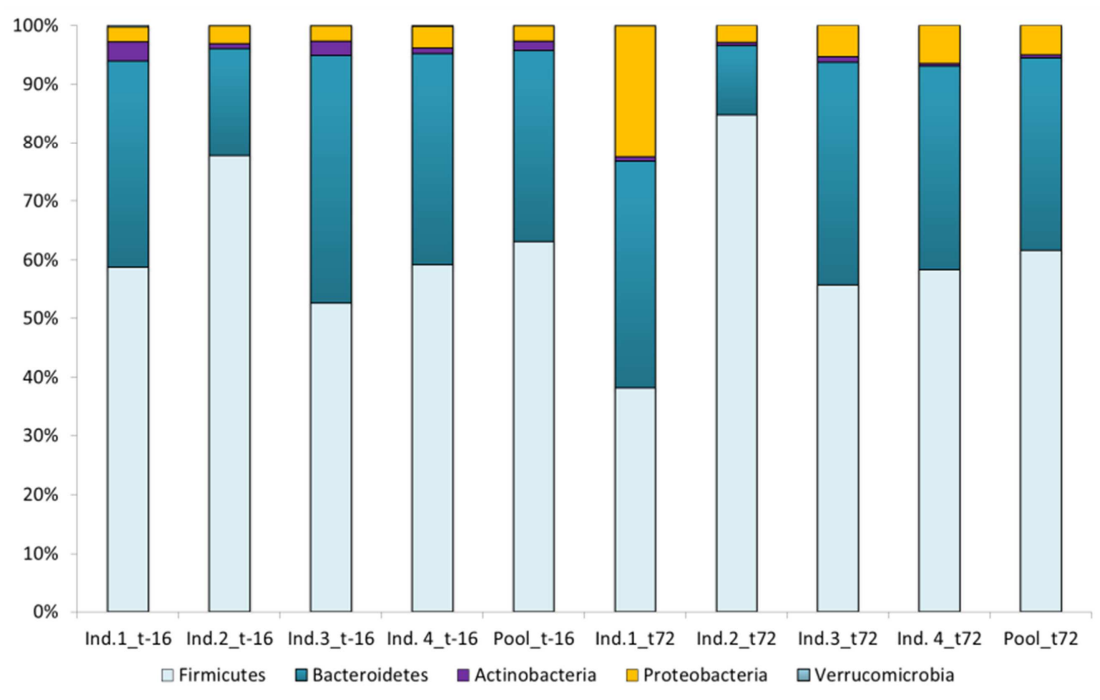

Fig. S4. Phyla level abundance (\%) analyzed in the fermentations with the different inocula at $\mathrm{t}_{-16}$ and $\mathrm{t}_{72}$. 


\section{Chapter 3}

\section{Evaluation of an optimal preparation of human standardized fecal inocula for in vitro fermentation studies}

Published as:

Aguirre M, Eck A, Koenen ME, Savelkoul PHM, Budding AE, Venema K (2015) Evaluation of an optimal preparation of human standardized fecal inocula for in vitro fermentation studies J Microbiol Methods 2015, 117: 78-84 


\section{Abstract}

This study investigated the optimal preservation approach to prepare human feces as inoculum for in vitro fermentations as an alternative to the use of fresh feces. The four treatments studied were: Treatment 1) fresh feces resuspended in dialysate solution + glycerol; Treatment 2 ) fresh feces resuspended in dialysate solution + glycerol and then stored at $-80{ }^{\circ} \mathrm{C}$; Treatment 3) fecal sample frozen with $1.5 \mathrm{~g}$ glycerol; and Treatment 4) fecal sample frozen. All the treatments contained $8.75 \mathrm{~g}$ of feces, $3.5 \mathrm{ml}$ dialysate and $4.9 \mathrm{ml}$ glycerol when inoculated in the TIM-2 in vitro system. Treatment 1 (fresh fecal preparation) was used as a reference.

The effects were evaluated in terms of i) metabolic activity and ii) composition of the microbiota using fermentation experiments in the TIM-2 in vitro system. In all treatments, high levels of acetate were produced followed by $n$-butyrate and propionate. However, the metabolic activity of the bacteria, in terms of short-chain fatty acid production, was affected by the different treatments. Microbiota composition was analyzed using the IS-pro profiling technique. Diversity in Actinobacteria, Firmicutes, Fusobacteria and Verrucomicrobia and Proteobacteria groups seemed to be preserved in all treatments whereas it was observed to decline in the Bacteroidetes group. Preparing a human fecal inoculum resuspended in dialysate solution with glycerol and then stored at $-80{ }^{\circ} \mathrm{C}$ showed high similarities to the results obtained with fresh feces, and is proposed as the optimal way to freeze fecal material as an alternative to fresh feces for in vitro fermentation studies. 
Optimal preparation of human standardized fecal inocula

\section{Introduction}

The human gut harbors a community of microorganisms commonly known as the microbiota. This community is dominated by anaerobic bacteria and consists of at least $10^{14}$ members with a wide variety of species $( \pm 500-1000)$ [1].

The intestinal microbiota in humans has been demonstrated to be highly active and able to ferment indigestible compounds from the host's diet [2]. The type of diet determines whether the fermentation process occurring in the gut is predominantly saccharolytic or proteolytic [3]. The metabolites from these two types of fermentation include mainly short-chain fatty acids (SCFA), specifically acetate, propionate and $n$-butyrate [2], and branched-chain fatty acids (BCFA) including principally iso-butyric, iso-valeric and 2-methylbutyric acids [4]. Metabolites such as acetate, propionate and butyrate are of particular interest since they have been found to be involved in lipid metabolism, reduction of food intake, improvement of tissue insulin sensitivity and intestinal barrier, and energy balance [5-10]. As a consequence, increasing evidence shows that the composition and activity of the intestinal microbiota is associated with the overall health state of humans, including obesity. Food components affect the composition and activity of the gut microbiota. Therefore, the fermentation characteristics of an ample number of substrates have been studied [11-13].

Part of these studies include experiments performed in in vitro systems which offer a high flexibility in their design since there are less limitations in regard to costs and ethical constraint when compared to human trials. For such in vitro studies, the use of a well-preserved fecal sample or inoculum is crucial to perform reproducible experiments and to guarantee the robustness and reliability of these experiments. The use of a standardized inoculum provides the opportunity to perform a large number of studies with the same microbiota for different substrates. This contributes to more reproducible assays that can be performed over a long period of time, which is impossible with a single fresh fecal sample.

There is a lack of literature addressing the possible variations in the microbial activity and composition induced by storage and preparation of a human fecal inoculum for in vitro studies. In experiments performed in rumen fluid, canine, and equine feces, freezing has been found to damage and disrupt the bacterial cell membrane, which causes the release of intracellular contents that subsequently led to loss of (members of the) communities [14-17]. Moreover, some groups of bacteria have been observed to be seriously damaged after freezing and thawing such as certain Gram-negative bacteria [16]. Alterations in the kinetics of fermentation as well as production of gases have also been found to occur after manipulation [16,18]. However, these negative effects were not observed during 
the preparation of the human fecal inoculum by Rose et al. [17] which, to our knowledge, is one of the few studies that have validated the use of fresh and frozen human microbiota. These authors observed that the viable cells in the microbiota stored for 44 weeks at $-80{ }^{\circ} \mathrm{C}$ were not affected and the microbial diversity of this inoculum did not substantially differ from a fresh one, although in that previous study the direct comparison between a frozen and fresh inoculum were not performed as the main goal. As explained before, there is a lack of information about an appropriate treatment to preserve human feces for in vitro fermentation experiments. Furthermore, these previous findings need to be expanded. Thus, the purpose of this study was to determine the optimal conditions to prepare a human fecal inoculum to be used in the TNO dynamic in vitro proximal colon model (TIM-2) [19]. Four different treatments to prepare human fecal inocula were studied and their efficacy was evaluated by monitoring the composition and activity (in terms of SCFA and BCFA production) of the microbiota under standard fermentation experiments. A potential alternative to fresh feces was successfully found.

\section{Material and methods}

\section{Fecal samples}

Participants involved in this study were non-smokers and had not used antibiotics, prebiotics, probiotics or laxatives 3 weeks prior to the donation. Fresh fecal samples were directly collected in a closed box containing an anaerobic strip (AnaeroGen ${ }^{\mathrm{TM}}$, Oxoid, Cambridge, UK). Donations and treatment preparations were handled under strict anaerobic conditions in an anaerobic chamber (Bactron IV, Sheldon manufacturing, Cornelius, OR USA) containing $5 \% \mathrm{H}_{2}, 5 \% \mathrm{CO}_{2}$, and $90 \% \mathrm{~N}_{2}$. A pool of feces was prepared from a group of 4 healthy volunteers (age range 29-62; 3 males, 1 female) recruited at TNO (Zeist, The Netherlands). Pooling has been previously shown to not drastically affect microbiota composition or activity [20].

The pooled fecal slurry was divided into four aliquots to prepare the different treatments as follows (Fig. 1): Treatment 1) fresh fecal slurry resuspended in dialysate solution + glycerol, this inoculum was tested right upon preparation; Treatment 2) fresh fecal slurry resuspended in dialysate solution + glycerol, frozen, stored and resuscitated prior to testing; Treatment 3) fresh fecal slurry frozen with $1.5 \mathrm{~g}$ glycerol, stored, resuscitated and, prior to testing, resuspended in dialysate solution + portion of glycerol; and Treatment 4) the fecal sample was frozen, stored, resuscitated and, prior to testing, resuspended in dialysate solution + glycerol. All inocula (Treatments $2-4)$ that were stored $\left(-80{ }^{\circ} \mathrm{C}, 1\right.$ week) were 
Optimal preparation of human standardized fecal inocula

immediately snap frozen in liquid nitrogen $\left(-196{ }^{\circ} \mathrm{C}\right)$ following their preparation and were resuscitated by immersion in a water bath $\left(37^{\circ} \mathrm{C}, 1 \mathrm{~h}\right)$ before parallel testing. Freezing in liquid nitrogen was performed in order to guarantee cell viability [21,22]. All the treatments contained $8.75 \mathrm{~g}$ of feces, $3.5 \mathrm{ml}$ dialysate and $4.9 \mathrm{~g}$ glycerol when inoculated in TIM-2. Treatment 1 (fresh fecal preparation) was used as a reference. The dialysate preparation has been previously described by Maathuis et al. [23]. The dialysate content per liter was as follows: $2.5 \mathrm{~g}$ $\mathrm{K}_{2} \mathrm{HPO}_{4} \bullet 3 \mathrm{H}_{2} \mathrm{O}, 4.5 \mathrm{~g} \mathrm{NaCl}, 0.005 \mathrm{~g} \mathrm{FeSO}_{4} \cdot 7 \mathrm{H}_{2} \mathrm{O}, 0.5 \mathrm{~g} \mathrm{MgSO}_{4} \bullet \mathrm{H}_{2} \mathrm{O}, 0.45 \mathrm{~g}$ $\mathrm{CaCl}_{2} \bullet 2 \mathrm{H}_{2} \mathrm{O}, 0.05 \mathrm{~g}$ ox bile and $0.4 \mathrm{~g}$ cysteine hydrochloride. The dialysate preparation included bile salts in order to partly reproduce the environment that the gut microbiota is usually exposed to in the colon [24]. Therefore, bile salts were included in the preparation of the different treatments to keep the tolerance of the cells. The impact of bile in fresh and freeze-dried prepared axenic cultures has been tested before [25] but not in fecal samples constituting a complex microbiota.

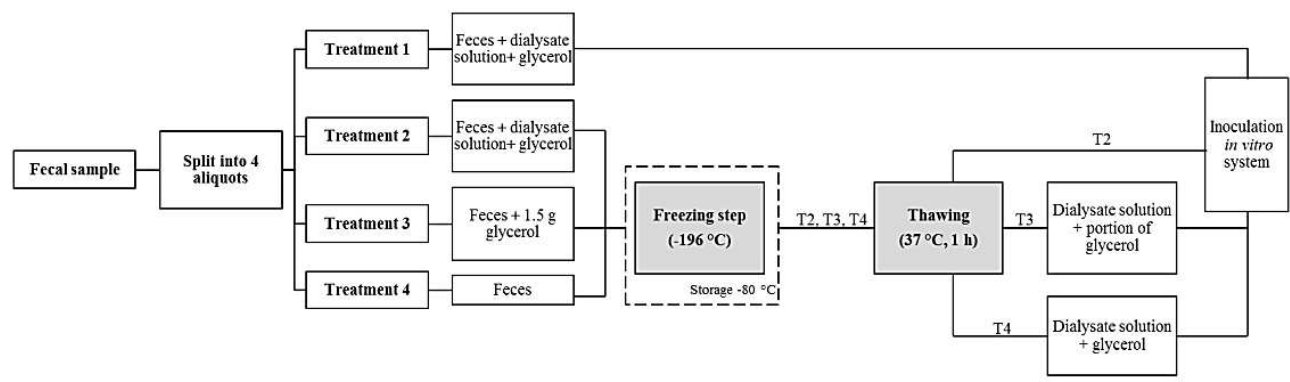

Fig. 1. Treatments for the different preparations of the inocula. Samples were frozen immediately, or mixed with glycerol and/or dialysate solution before freezing in liquid nitrogen. All the treatments contained $8.75 \mathrm{~g}$ of feces, $3.5 \mathrm{ml}$ dialysate and $4.9 \mathrm{~g}$ glycerol when inoculated in TIM-2.

\section{Standard Ileal Efflux Medium (SIEM)}

A growth medium prepared with complex, indigestible carbohydrates (pectin, xylan, arabinogalactan, amylopectin and starch), protein, vitamins, Tween 80 and bile (Tritium Microbiology; Veldhoven, The Netherlands) was used to feed the bacteria at a rate of $2.5 \mathrm{ml} / \mathrm{h}$ during fermentation experiments. Specific details about this SIEM preparation have been previously described $[23,26]$.

\section{In vitro fermentation}

The TNO in vitro model of the proximal colon (TIM-2) has been described in detail before $[17,19,26]$. The system was flushed with $\mathrm{N}_{2}$ before the introduction of 
the inoculum and was maintained under this condition at $37^{\circ} \mathrm{C}$ for $96 \mathrm{~h}$ with the $\mathrm{pH}$ kept at or above 5.8 by automatic titration with $2 \mathrm{M} \mathrm{NaOH}$. To remove water and fermentation products from the lumen, a dialysis system (described in Maathuis et al. [23]) ran through the lumen.

After 24 and $48 \mathrm{~h}$ of fermentation, a $25 \mathrm{ml}$ lumen sample was removed from the system to mimic the transit of material from the proximal to the distal colon [23].

\section{Design of the study}

A $30 \mathrm{ml}$ portion of fecal homogenate was used to inoculate four units of TIM-2 separately. Each unit was then filled to $120 \mathrm{ml}$ with $90 \mathrm{ml}$ of dialysate and maintained at this volume by a level sensor (liquiphant FTL20-0025, Endress+Hauser).

Immediately after the inoculation, the microbiota was left to adapt (16 h) to the new environment. After this adaptation period, the microbiota was deprived from any carbohydrate source (starvation) for $2 \mathrm{~h}$. The starvation period is performed in order to completely use the carbohydrates in SIEM [27] which provides the best condition to assess the effects of a test compound without bias of the SIEM component from the adaptation phase. Minekus et al. [28] explained that this starvation period was established when a lack of production of acids was observed in the system when the feeding line was turned off. This could be interpreted as the depletion of the fermentable carbohydrate.

After the starvation period, units were fed with SIEM until the end of the experiment. Samples were taken from the lumen before $\mathrm{t}=0(-16,-14$ and $-8 \mathrm{~h})$ and after $\mathrm{t}=0(24,48$ and $72 \mathrm{~h})$. Dialysate was collected after $0,24,48$ and $72 \mathrm{~h}$.

\section{Analytical methods}

With samples from the lumen and dialysate, the cumulative production of SCFA and BCFA was calculated. Microbial composition was profiled using IS-pro (see below) in all samples from the lumen.

\section{Bacterial metabolites}

Analysis of SCFA (acetate, propionate, and butyrate) and BCFA (iso-butyrate and iso-valerate)

Samples were prepared and analyzed as described previously [26]. Briefly, samples were centrifuged (12000 r.p.m. at $4{ }^{\circ} \mathrm{C}$ for $10 \mathrm{~min}$ ). A mixture of formic acid (20\%), methanol, and 2-ethyl butyric acid (internal standard, $2 \mathrm{mg} / \mathrm{ml}$ in methanol) was added to the supernatant. Samples were measured by gas-chromatography (Stabilwax-DA, length $15 \mathrm{~m}$, ID $0.53 \mathrm{~mm}$, film thickness $0.1 \mathrm{~mm}$; Varian Chrompack, Bergen op Zoom, The Netherlands) after acidification. Standard 
Optimal preparation of human standardized fecal inocula

curves were obtained by injecting calibrated quantities of a blend of volatile fatty acids and amounts were calculated from the graph obtained correlating peak height and time measured (all reagents from Sigma-Aldrich with the exception of formic acid which was from Merck).

\section{Characterization of bacterial population using IS-profiling \\ DNA isolation}

DNA was extracted from the luminal samples using the easyMAG extraction kit according to the manufacturer's instructions (Biomérieux, Marcy l'Etoile, France). In brief, $200 \mu \mathrm{l}$ of lumen was placed in an Eppendorf tube. Then, $400 \mu \mathrm{l}$ of nucliSENS lysis buffer was added. This content was vortexed and further centrifuged (14000 rpm; $2 \mathrm{~min}), 100 \mu \mathrm{l}$ of the preparation was transferred to an easyMAG isolation boat containing $2 \mathrm{ml}$ of nucliSENS lysis buffer. This suspension was incubated for $10 \mathrm{~min}$ at room temperature and $70 \mu \mathrm{l}$ of magnetic silica beads were added. The easyMAG automated DNA isolation machine was used following the "specific A" protocol, and the lysed workflow and elution with $110 \mu \mathrm{l}$ buffer for further DNA extraction. DNA was diluted 10 times before proceeding with using the IS-pro Kit.

16S-23S intergenic spacer (IS) profiling of the microbiota

Amplification of IS-regions was performed with the IS-pro assay (IS-diagnostics, Amsterdam, The Netherlands). IS-pro differentiates bacterial species by the length of the 16S-23S rDNA intergenic spacer (IS) region with taxonomic classification by phylum-specific fluorescently labeled PCR primers. The procedure consists of two multiplex PCRs: a first PCR has two different fluorescent colored labels. One for the phyla Actinobacteria, Firmicutes, Fusobacteria and Verrucomicrobia (further abbreviated as Act/Fir/Fus/Verr) and a second color for the phylum Bacteroidetes. A separate PCR is performed for the phylum Proteobacteria. The assay was performed according to the protocol provided by the manufacturer. Amplifications were carried out on a GeneAmp PCR system9700 (Applied Biosystems, Foster City, CA). After PCR, $5 \mu$ l of PCR product was mixed with $20 \mu \mathrm{l}$ formamide and $0.2 \mu \mathrm{l}$ Mapmaker 1500 ROX-labeled size marker (BioVentures, Murfreesboro, TN, USA). DNA fragment analysis was performed on an ABI Prism 3500 Genetic Analyzer (Applied Biosystems).

Data analysis

Pre-processing was carried out by the IS-pro proprietary software suite (ISDiagnostics, Amsterdam, The Netherlands) and resulted in microbial profiles. Each profile consisted of a set of color-labelled peaks, with each peak related to a 
specific IS fragment (measured in nucleotides) and the color to a specific phylum group (Act/Fir/Fus/Verr, Bacteroidetes or Proteobacteria). The intensity of peaks reflected the quantity of PCR product (measured in relative fluorescent units). As discussed above, a bacterial species was characterized by a specific set of peaks. For simplicity, we considered each peak as an operational taxonomic unit (OTU) and its corresponding intensity as abundance, as these were previously shown to be highly correlated $[29,30]$.

Diversity analysis

Diversity was calculated per phylum using the Shannon index that was recently shown to be a robust estimation of microbial diversity [31]. Dissimilarities between samples were calculated as the cosine distance between each pair of bacterial profiles. These were summarized and visualized in a low-dimensional space using principal coordinate analysis (PCoA). OTUs with at least a $\log _{2}$ intensity $>13$ (intensity $>8192 \mathrm{RFU}$ ) were selected and represented using a relative abundance visualization.

\section{Data presentation}

The in vitro fermentation model is computer controlled and over the past two decades it has been shown to be highly reproducible [17,32,33]. Therefore, the experiments were performed in series of one per treatment $(n=1)$. To avoid unnecessary repetition, this is not indicated further in the text or graphs in the results section. Since the sample size is small no statistical analysis was executed. However, for treatments 2, 3 and 4 the ratio between the metabolites produced at each sampling time point was calculated with respect to the metabolic values corresponding to each sampling time point of treatment 1 which was considered as a reference (i.e., $t_{-16}$ treatment $2 / t_{-16}$ treatment 1 and so forth). A value equal to 1 indicates no change; a value of $>1$ indicates an increase; and a value of $<1$ indicates a decrease of the respective metabolite.

\section{Results}

The use of a freshly collected fecal sample is the optimal way to guarantee minimal perturbation of a microbial inoculum for in vitro fermentation experiments. Therefore, Treatment 1 (fresh fecal preparation) was considered as a reference in the study. 
Optimal preparation of human standardized fecal inocula

\section{Microbiota activity \\ SCFA}

Despite the similarities in the cumulative amounts of SCFA, differences between the treatments occurred in the fermentation kinetics during the entire experimental period. Fig. 2 shows similarity in kinetics of SCFA production between Treatment 1 (reference) and Treatment 2 whereas Treatment 3 and 4 are different when compared with these first two but similar to each other.

The total cumulative amounts and ratios of SCFA produced in the TIM- 2 system after $72 \mathrm{~h}$ fermentation with the simulated standard media were similar between the 4 differently treated inocula (Fig. S1 and S2). Inocula prepared under Treatment 1 produced $210.3 \mathrm{mmol}$ SCFA after $72 \mathrm{~h}$, followed by Treatment $4(210.2 \mathrm{mmol})$, Treatment 2 (201.5 mmol) and Treatment 3 (198.4 mmol). Small differences in acetate and in total SCFA production were observed in Treatment 2 as compared to Treatment 1. Treatment 3 showed lower amounts of propionate and total SCFA than Treatment 1. Treatment 4 also presented lower propionate amounts when compared to Treatment 1.

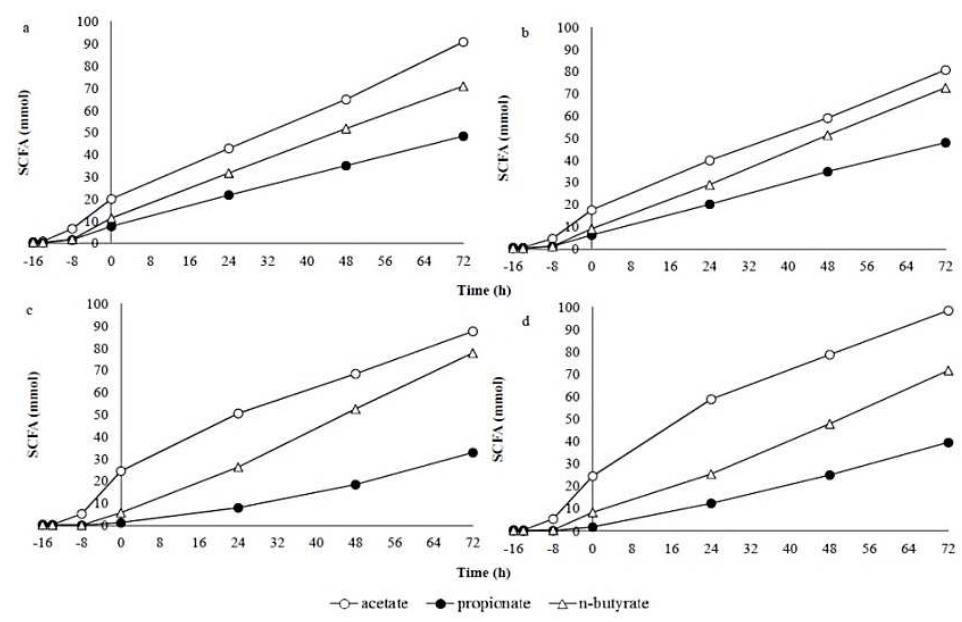

Fig. 2. Kinetics of cumulative production of SCFA during the $72 \mathrm{~h}$ experimental time. Samples were taken in time and analyzed by gaschromatography. The cumulative production of each individual SCFA (acetate, propionate and butyrate) and their sum (total) was calculated using the concentration from the GC analysis and volumes of the compartments of the model (lumen and dialysate). (a) Treatment 1: feces mixed with dialysate and glycerol; (b) Treatment 2: feces mixed with dialysate and glycerol and then frozen; (c) Treatment 3: feces mixed with glycerol and frozen; (d) Treatment 4: feces frozen immediately. 


\section{BCFA}

The fermentation experiments with inocula prepared under Treatment 1 resulted in a higher total cumulative production of BCFA when compared with the other treatments (Fig. 3). In all treatments less $i$-butyrate was produced than $i$-valerate. The total cumulative BCFA production of the inoculum prepared under Treatment 2 was lower than it was in Treatment 1. Treatment 3 and Treatment 4 total cumulative BCFA values were also lower when compared to Treatment 1 .

The same similarities observed in the fermentation kinetics for SCFA (emergence of two distinct groups: Treatments 1 and 2 different from Treatments 3 and 4) were also observed for BCFA (Fig. S3).

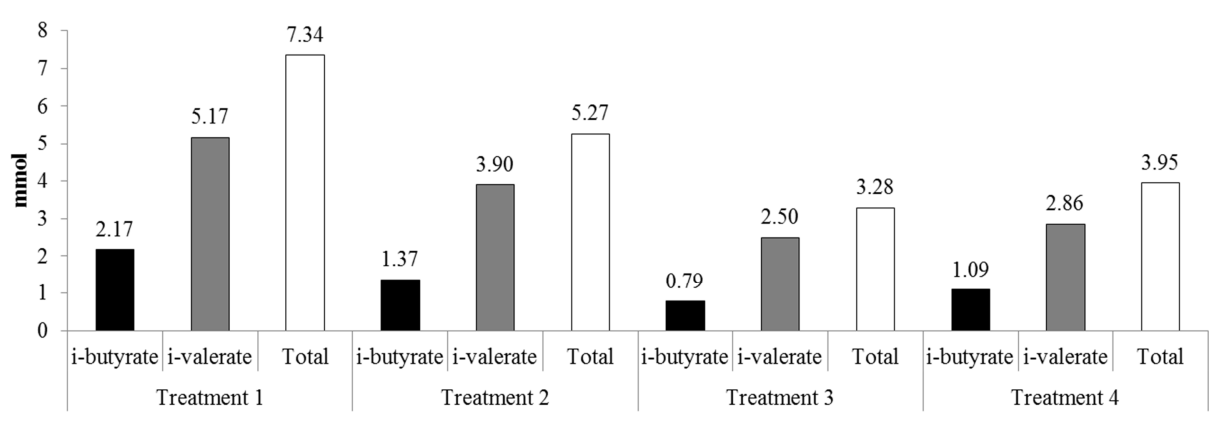

Fig. 3. Cumulative BCFA production $(\mathrm{t}=72)$ from the fermentations. Samples were analyzed as described in the legend of Fig. 2. The cumulative amount produced after $72 \mathrm{~h}$ incubation is shown. Treatment 1: feces mixed with dialysate and glycerol; Treatment 2: feces mixed with dialysate and glycerol and then frozen; Treatment 3: feces mixed with glycerol and frozen; Treatment 4: feces frozen immediately.

\section{Characterization of bacterial composition by IS-profiling}

First, an analysis was done on the diversity of each phylum group over time (Fig. S4).

In general, diversity seemed to be affected most during the adaptation period. Despite this, two distinct groups (Treatments 1 and 2; Treatments 3 and 4) were formed based on the similarities on the diversity indices and the shifts in OTUs over time in agreement with what we observed for microbiota activity.

After inoculation, diversity decreased for all phyla for all treatments until $t=-8$. For Treatment groups 1 and 2 there is a marked increase in diversity for all phyla during the period from $t=-8$ to $t=0$. For Treatments 3 and 4 this effect is less outspoken (Act/Fir/Fus/Verr) or absent (Bacteroidetes and Proteobacteria). The whole procedure that the microbiota was exposed to (e.g., inoculation, conditions 
Optimal preparation of human standardized fecal inocula

in the system, medium, etc.) showed to be less favorable for Bacteroidetes for all treatments. This was shown by a decrease in diversity over time when compared to Act/Fir/Fus/Verr and Proteobacteria in which these effects were less severe.

Shifts in OTUs over time are highly similar for Treatments 1 and 2 on the one hand and for Treatments 3 and 4 on the other, thus forming two distinct groups (Fig. S4 and S5). In the case of Act/Fir/Fus/Verr, the final diversity index ( $t=72)$ decays in Treatment 1 when compared to Treatments 2, 3 and 4. In Bacteroidetes, it is Treatment 3 which presents the lowest diversity at $t=72$. Interestingly, Treatments 2 and 4 show the higher diversity at this sampling time in this phylum (diversity index: 2.53 and 2.52 , respectively).

Treatment 1 exhibits a higher diversity in Proteobacteria when compared to Treatments 2, 3, and 4 at the end of the study.

This grouping was confirmed when differences in overall bacterial community composition were assessed using cosine distances between the bacterial profiles of the samples and visualized in a PCoA plot (Fig. 4). These distances, often referred to as beta-diversity, quantify the compositional dissimilarity between sets of samples. This type of analysis is used extensively to compare different microbial communities. The PCoA visualization showed samples that were prepared under Treatments 1 and 2 were segregated from samples that were prepared under Treatments 3 and 4. Also, when observed through time, samples from Treatments 1 and 2 were grouped closely together at each time point confirming the similarities that were observed at the metabolite level.

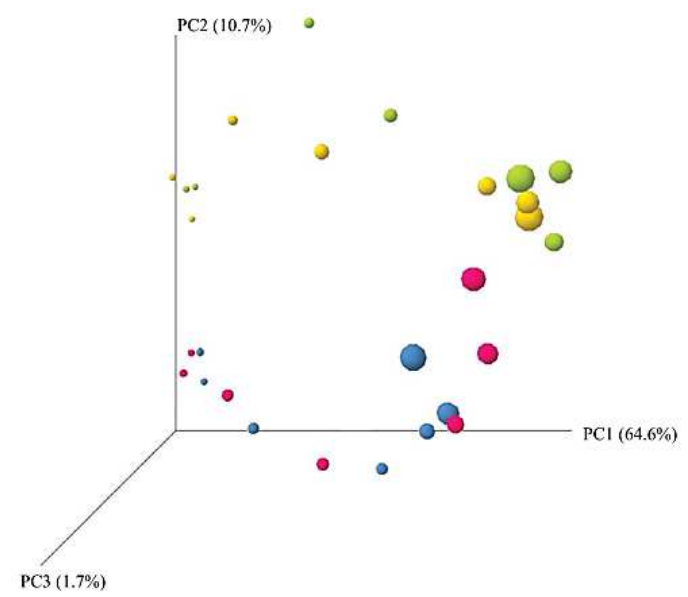

Fig. 4. PCoA plot of the treatments. The three principal coordinates from the PCoA analysis of the cosine distances that were calculated between the bacterial compositions of the samples. Each color corresponds to a Treatment group (blue: T1; pink: T2; yellow: T3; green: T4. The variance explained by the PCs is indicated in 
parentheses on the axes. The size of the groups increases in order to represent the different sampling times.

\section{Discussion}

Freshly collected fecal samples may not always be available. In some circumstances, the use of fresh human microbiota is not possible because donors live far away from the laboratory or because they are not continuously available to repeatedly participate at various times during the study. In addition, the composition and activity of repeated donations are likely to be different from day to day. Therefore, these samples are not always identical.

To guarantee a constant inoculum over time for different studies, most studies use prepared and stored inocula. In these cases, the use of frozen feces provides more flexibility for this type of experiments. However, preparation and storage of fecal samples have been shown to impact microbial composition, viability and activity [14-16]. It is unknown to what extent various preparation and storage methods impact the microbiota and what preservation method is best. Hence, the effects of different treatments used to prepare human feces as inocula for in vitro fermentation experiments must be studied before any preservation method is chosen as an alternative to the use of fresh feces. For this reason, the aim of this study was to find the optimal way to prepare an inoculum for in vitro studies that could be stored for future experiments. Treatments were selected based upon routinely used materials (i.e. glycerol, saline suspension, vitamins, and minerals) and procedures (deep freezing in liquid nitrogen and storage at $-80{ }^{\circ} \mathrm{C}$ ) in a lab acquainted with preparing long-term stocks of fecal suspensions. The use of a saline preparation is common in the homogenization of slurry for fecal transplants $[34,35]$. Furthermore, for technical practicalities of working with stocks of gut microbiota, dialysate (a composite previously described Maathuis et al. [23]) was selected to prevent the cells from osmotic shock/stress . Glycerol has also been recommended when creating stocks of bacterial cultures [36] as well as a cryoprotectant capable of preserving the viability of cells [37]. And importantly, freezing regime in liquid nitrogen has been shown to provide a maximum recovery of cells [38]. The effects of the treatments were evaluated in terms of metabolic activity and composition of the microbiota using fermentation experiments in the TIM-2 in vitro system with Treatment 1 (fresh fecal preparation) as a reference.

\section{Microbiota activity}

Metabolic functions of the microbiota have been found to be influenced by a wide range of factors including temperature and the preservation media used [39]. 
Optimal preparation of human standardized fecal inocula

Functions such as the deconjugation of glycosylated metabolites, an essential activity of the gut microbiota, can be especially altered by changes in temperature [40,41]. Flores et al. [39] observed that enzymatic activity of $\beta$-glucuronidase decayed after freezing and thawing. In regard to the preservation media, bacteria kept in glycerol have been shown to rapidly use it as a source of energy after thawing [14]. Rémond et al. [42] demonstrated the utilization of glycerol and the consequent increase in $n$-butyrate proportions that occur at the expense of acetate. For this reason, freezing with glycerol has been recommended as a treatment to maintain the diversity in a bacterial community, but it has been considered inadequate for fermentation studies [14].

Despite the changes in the metabolic function observed among the treatments, it is important to consider the similarities between Treatments 1 and 2 in the ratios and the kinetics for SCFA and BCFA production throughout the experiment (Fig. 2, S1, S3, Table S1). This suggests that processing the inoculum with Treatment 2 did improve activity recovery in contrast with earlier findings where the use of a cryoprotectant to fecal biomass did not enhance functionality in the mixed community [43]. The functional recovery of the community could be the result of functional overlap in the microbiota. This could be explained by the fact that different bacteria in a community share metabolites with their partners in crossfeeding such as acetate [44], glucose [45,46], fixed nitrogen [45], amino acids [47] or hydrogen [48] as well as by the fact that there is a tremendous functional overlap in the multiple pathways dedicated to production of e.g., organic acids.

The relative high BCFA production in Treatment 2 compared to Treatments 3 and 4 (Fig. 3, S3) demonstrates that the microbes which have the ability to catabolize more protein compounds are better preserved in this treatment.

\section{Characterization of bacterial population by IS-profiling}

Diversity was affected during the $16 \mathrm{~h}$ adaptation period (Fig. S4). The changes observed are suggested to be influenced by different factors such as thawing, redox potential, and osmotic stress caused by the change of environment (from human gut to in vitro model).

Firstly, the inoculum was thawed at $37{ }^{\circ} \mathrm{C}$ during $1 \mathrm{~h}$. This condition could have influenced the stability of the microbial community since it has been found that DNA can be fragmented when thawing during this period of time [49]. Cardona $e t$ al. [49] observed that abundance of certain bacterial taxa was affected between frozen and unfrozen samples which were thawed for 1 to $3 \mathrm{~h}$, especially in the Bacteroides genus.

Secondly, it may be that the redox potential (Eh) in the system may have influenced the stability of the community during the experiment in all treatments. 
The Eh range in the TIM-2 lumen is within -200 to $-300 \mathrm{mV}$, similar to physiological values [50]. Although some reducing and buffering agents are used in the system, reaching an Eh close to $-300 \mathrm{mV}$ is not rapidly achieved during the adaptation period (unpublished data). Obligate anaerobes require a reducing environment to be metabolically active [50] since the redox potential influences the activity of their enzymes involved in the reduction and oxidation of compounds. Therefore, an inappropriate redox potential in the environment could alter the performance of their metabolic functions affecting the growth of these bacteria and their survival.

Thirdly, the change of environmental conditions encountered by the bacteria in terms of different solute concentrations (substrates, salts, among others) may cause water to be drawn out of the cells. Many cells are sensitive to this phenomenon known as osmotic shock/stress. The use of hypertonic solutions such as $\mathrm{NaCl}$ (which is also contained in the dialysate preparation used in this study) has been found to reduce this kind of stress on bacteria. Nevertheless, $\mathrm{NaCl}$ has been found to cause surface lesions which could be ameliorated by the addition of glycerol. Glycerol also protects against osmotic shock, it decreases the freezing-point of biological fluids and water by colligative action and it prevents eutectic crystallization. Furthermore, membrane vesicles containing enzymes are protected by glycerol [36]. However, the prevention of loss of viable cells by glycerol was shown not to be equal for all members of a diverse and complex community characteristic for a fecal sample [37]. Therefore, in this study we decided to include a preparation with a low or absent concentration of glycerol (treatments 3 and 4) which showed that, irrespective of the presence of this cryoprotectant, bacterial diversity was reduced by the damaging effects of processing the fecal sample as previously also observed by others [37].

It is important to realize that despite the different factors mentioned above that could have influenced the composition of the microbiota in the TIM-2 system, it has been observed that this model is highly reproducible. The TIM-2 system has been demonstrated to preserve the composition of the microbiota similar to its original source since Kovatcheva-Datchary et al. [51], showed that the composition of the microbiota from (fresh) feces and TIM-2 samples could not be distinguished. In addition, microbial activity and composition can be successfully reproduced throughout several weeks using the model as confirmed by performing fermentation experiments using inocula from two different groups of donors (lean and obese). These experiments showed a high similarity within the groups in all the measured parameters forming two different groups depending on the microbiota used [52]. 
Optimal preparation of human standardized fecal inocula

Despite the diversity being similar for some samples over time (e.g., for Proteobacteria for Treatment 1 at $\mathrm{t}=0$ and $72 \mathrm{~h}$; Shannon index around 3.3), this does not mean that the microbiota composition at these time points was the same (Fig. S5 - Proteobacteria). Kerckhof et al. [43] also observed no significant differences in overall community structure in a fecal community treated with several cryopreservation protocols based on a diversity index. However, the authors did observe that not all OTUs were preserved. Therefore, choosing a specific preservation technique in this case must be based on the importance of preserving single OTUs or the community structure as a whole for the study.

In general, diversity in Act/Fir/Fus/Verr and Proteobacteria seemed to be preserved in Treatments 2, 3, and 4 while it is observed to decline in Bacteroidetes. Still, the shifts in the composition of the microbiota due to death of microbial species and the selective growth appeared to be similarly overcome in Treatments 1 and 2 and in Treatments 3 and 4, forming two distinct groups (Fig. 4 and S4). In contrast to earlier findings where there was no clear effect of several cryopreservation protocols on taxonomic groups in a human fecal community [43], we found in this study that Treatment 2 was an optimal treatment to preserve the characteristic phylogenetic groups in a fecal inoculum resembling the closest to the reference (fresh feces).

\section{Conclusion}

We conclude that preparing a human fecal inoculum resuspended in dialysate solution with glycerol and then stored at $-80{ }^{\circ} \mathrm{C}$ after snap-freezing in liquid nitrogen (Treatment 2) is a viable alternative to fresh feces (resuspended in the same buffer) for in vitro fermentation studies.

Further experiments are recommended to i) test the optimal time and alternatives for thawing as e.g., discussed by Hamilton et al. [35] who tested thawing using an ice-bath, ii) study the extent of the effects of preparing a human fecal inoculum on in vitro experiments fermenting specific substrates and iii) determine the effect of different treatments on microbial enzyme activities and gas production.

\section{Acknowledgments}

The authors thank Malieka van der Lugt-Degen and Linda Poort for assisting during the ABI runs. This study was partly funded by the Top Institute Food \& Nutrition (GH004) (TIFN, Wageningen, The Netherlands). 


\section{References}

1. Bäckhed F, Hao D, Wang T, Hooper Lora V, Koh GY, et al. (2004) The gut microbiota as an environmental factor that regulates fat storage. Proc Natl Acad Sci U S A 101: 15718-15723.

2. Flint HJ, Duncan SH, Scott KP, Louis P (2007) Interactions and competition within the microbial community of the human colon: links between diet and health. Environ Microbiol 9: 1101-1111.

3. Scott KP, Gratz SW, Sheridan PO, Flint HJ, Duncan SH (2013) The influence of diet on the gut microbiota. Pharmacol Res 69: 52-60.

4. Bergman EN (1990) Energy contributions of volatile fatty acids from the gastrointestinal tract in various species. Physiol Rev 70: 567-590.

5. Al-Lahham S, Roelofsen H, Rezaee F, Weening D, Hoek A, et al. (2011) Propionic acid affects immune status and metabolism in adipose tissue from overweight subjects. Eur J Clin Invest 42: 357-364.

6. Al-Lahham S, H, , Roelofsen H, Priebe M, Weening D, Dijkstra M, et al. (2010) Regulation of adipokine production in human adipose tissue by propionic acid. Eur J Clin Invest 40: 401-407.

7. Ferchaud-Roucher V, Pouteau E, Piloquet H, Zair Y, Krempf M (2005) Colonic fermentation from lactulose inhibits lipolysis in overweight subjects. Am $J$ Physiol Endocrinol Metab 289: E716-E720.

8. Peng L, Li ZR, Green RS, Holzman IR, Lin J (2009) Butyrate enhances the intestinal barrier by facilitating tight junction assembly via activation of AMP-activated protein kinase in Caco-2 cell monolayers. J Nutr 139: 1619-1625.

9. Roediger W, E, (1982) Utilization of nutrients by isolated epithelial cells of the rat colon. Gastroenterology 83: 424-429.

10. Scheppach W (1994) Effects of short chain fatty acids on gut morphology and function. Gut 35: S35-S38.

11. Cardelle-Cobas A, Olano A, Corzo N, Villamiel M, Collins M, et al. (2012) In vitro fermentation of lactulose-derived oligosaccharides by mixed fecal microbiota. J Agric Food Chem 60: 2024-2032.

12. Fassler C, Arrigoni E, Venema K, Brouns F, Amadó R (2006) In vitro fermentability of differently digested resistant starch preparations. Mol Nutr Food Res 50: 1220-1228.

13. Hernot D, Boileau T, Bauer L, Middelbos I, Murphy M, et al. (2009) In vitro fermentation profiles, gas production rates, and microbiota modulation as affected by certain fructans, galactooligosaccharides, and polydextrose. $J$ Agric Food Chem 57: 1354-1361.

14. Prates A, de Oliveira J, Abecia L, Fondevila M (2010) Effects of preservation procedures of rumen inoculum on in vitro microbial diversity and fermentation. Anim Feed Sci Technol 155: 186-193. 
Optimal preparation of human standardized fecal inocula

15. Bosch G, Wrigglesworth D, Cone J, Pellikaan W, Hendriks W (2013) Effects of preservation conditions of canine feces on in vitro gas production kinetics and fermentation end products. J Anim Sci 91: 259-267.

16. Murray J-AM, McMullin P, Handel I, Hastie PM (2012) The effect of freezing on the fermentative activity of equine faecal inocula for use in an in vitro gas production technique. Anim Feed Sci Technol 178: 175-182.

17. Rose D, Venema K, Keshavarzian A, Hamaker B (2010) Starch-entrapped microspheres show a beneficial fermentation profile and decrease in potentially harmful bacteria during in vitro fermentation in faecal microbiota obtained from patients with inflammatory bowel disease. Br J Nutr 103: 15141524.

18. Pastorelli G, Faustini M, Attard E (2014) In vitro fermentation of feed ingredients by fresh or frozen pig fecal inocula. Anim Sci J 85: 690-697.

19. Venema K, Van Nuenen H, Smeets-Peeters M, Minekus M, Havenaar R (2000) TNO's in vitro large intestinal model: an excellent screening tool for functional food and pharmaceutical research. Ernährung/Nutrition 24: 558564.

20. Aguirre M, Ramiro-Garcia J, Koenen ME, Venema K (2014) To pool or not to pool? Impact of the use of individual and pooled fecal samples for in vitro fermentation studies. J Microbiol Methods 107C: 1-7.

21. Malik KA (1991) Cryopreservation of bacteria with special reference to anaerobes. World J Microb Biot 7: 629-632.

22. Day J, Pennington M, Stephen F (1995) Freeze-Drying and Cryopreservation of Bacteria. Cryopreservation and Freeze-Drying Protocols: Humana Press. pp. 21-30.

23. Maathuis A, Hoffman A, Evans A, Sanders L, Venema K (2009) The Effect of the Undigested Fraction of Maize Products on the Activity and Composition of the Microbiota Determined in a Dynamic in Vitro Model of the Human Proximal Large Intestine. J Am Coll Nutr 28: 657-666.

24. Ridlon JM, Kang DJ, Hylemon PB (2006) Bile salt biotransformations by human intestinal bacteria. J Lipid Res 47: 241-259.

25. Saarela M, Virkajarvi I, Alakomi HL, Mattila-Sandholm T, Vaari A, et al. (2005) Influence of fermentation time, cryoprotectant and neutralization of cell concentrate on freeze-drying survival, storage stability, and acid and bile exposure of Bifidobacterium animalis ssp. lactis cells produced without milkbased ingredients. J Appl Microbiol 99: 1330-1339.

26. Van Nuenen H, Meyer P, Venema K (2003) The effect of various inulins and Clostridium difficile on the metabolic activity of the human colonic microbiota in vitro. Microb Ecol Health Dis 15: 137-144. 
27. De Graaf A, Maathuis A, De Waard P, Deutz N, Dijkema C, et al. (2010) Profiling human gut bacterial metabolism and its kinetics using [U-13C] glucose and NMR NMR in Biomedicine 23: 2-12.

28. Minekus M, Smeets-Peeters M, Bernalier A, Marol-Bonnin S, Havenaar R, et al. (1999) A computer-controlled system to simulate conditions of the large intestine with peristaltic mixing, water absorption and absorption of fermentation products. Appl Microbiol Biotechnol 53: 108-114.

29. Budding AE, Grasman ME, Lin F, Bogaards JA, Soeltan-Kaersenhout DJ, et al. (2010) IS-pro: high-throughput molecular fingerprinting of the intestinal microbiota. FASEB J 24: 4556-4564.

30. Budding AE, Vandenbroucke-Grauls CM, Melles DC, van Duijkeren E, Kluytmans JA, et al. (2010) Binary IS typing for Staphylococcus aureus. PLoS One 5: e13671.

31. Haegeman B, Hamelin J, Moriarty J, Neal P, Dushoff J, et al. (2013) Robust estimation of microbial diversity in theory and in practice. ISME J 7: 10921101.

32. Van den Abbeele P, Venema K, Van de Wiele T, Verstraete W, Possemiers S (2013) Different Human Gut Models Reveal the Distinct Fermentation Patterns of Arabinoxylan versus Inulin. J Agric Food Chem 61: 9819-9827.

33. Maathuis AJH, van den Heuvel EG, Schoterman MHC, Venema K (2012) GalactoOligosaccharides Have Prebiotic Activity in a Dynamic in vitro Colon Model Using a C-Labeling Technique. J Nutr 142: 1205-1212.

34. Kleger A, Schnell J, Essig A, Wagner M, Bommer M, et al. (2013) Fecal transplant in refractory Clostridium difficile colitis. Dtsch Arztebl Int 110: 108-115.

35. Hamilton MJ, Weingarden AR, Sadowsky MJ, Khoruts A (2012) Standardized frozen preparation for transplantation of fecal microbiota for recurrent Clostridium difficile infection. Am J Gastroenterol 107: 761-767.

36. Hubalek Z (2003) Protectants used in the cryopreservation of microorganisms. Cryobiology 46: 205-229.

37. Waite D, Deines P, Taylor M (2013) Quantifying the impact of storage procedures for faecal bacteriotherapy in the critically endangered New Zealand parrot, the kakapo (Strigops habroptilus). Zoo Biol 32: 620-625.

38. Dan M, Richardson J, Miliotis MD, Koornhof HJ (1989) Comparison of preservation media and freezing conditions for storage of specimens of faeces. J Med Microbiol 28: 151-154.

39. Flores R, Shi J, Gail MH, Ravel J, Goedert JJ (2012) Assessment of the human faecal microbiota: I. Measurement and reproducibility of selected enzymatic activities. Eur J Clin Invest 42: 848-854.

40. Nakamura J, Kubota Y, Miyaoka M, Saitoh T, Mizuno F, et al. (2002) Comparison of four microbial enzymes in Clostridia and Bacteroides isolated from human feces. Microbiol Immunol 46: 487-490. 
Optimal preparation of human standardized fecal inocula

41. Renwick AG, Tarka SM (2008) Microbial hydrolysis of steviol glycosides. Food Chem Toxicol 46 Suppl 7: S70-74.

42. Rémond B, Souday E, Jouany JP (1993) In vitro and in vivo fermentation of glycerol by rumen microbes. Anim Feed Sci Technol 41: 121-132.

43. Kerckhof F-M, Courtens ENP, Geirnaert A, Hoefman S, Ho A, et al. (2014) Optimized Cryopreservation of Mixed Microbial Communities for Conserved Functionality and Diversity. PLOS ONE 9: e99517.

44. Rozen DE, Philippe N, Arjan de Visser J, Lenski RE, Schneider D (2009) Death and cannibalism in a seasonal environment facilitate bacterial coexistence. Ecol Lett 12: 34-44.

45. Kim HJ, Boedicker JQ, Choi JW, Ismagilov RF (2008) Defined spatial structure stabilizes a synthetic multispecies bacterial community. PNAS 105: 1818818193.

46. Gore J, Youk H, van Oudenaarden A (2009) Snowdrift game dynamics and facultative cheating in yeast. Nature 459: 253-256.

47. Shou W, Ram S, Vilar JM (2007) Synthetic cooperation in engineered yeast populations. Proc Natl Acad Sci U S A 104: 1877-1882.

48. Hillesland KL, Stahl DA (2010) Rapid evolution of stability and productivity at the origin of a microbial mutualism. Proc Natl Acad Sci U S A 107: 2124-2129.

49. Cardona S, Eck A, Cassellas M, Gallart M, Alastrue C, et al. (2012) Storage conditions of intestinal microbiota matter in metagenomic analysis. BMC Microbiol 12: 158.

50. Wilson M (2005) Microbial Inhabitants of Humans: Their Ecology and Role in Health and Disease: Cambridge University Press.

51. Kovatcheva-Datchary P, Egert M, Maathuis A, Rajilic -Stojanovic M, De Graaf AA, et al. (2009) Linking phylogenetic identities of bacteria to starch fermentation in an in vitro model of the large intestine by RNA-based stable isotope probing. Environ Microbiol 11: 914-992.

52. Aguirre M, Jonkers DM, Troost FJ, Roeselers G, Venema K (2014) In vitro characterization of the impact of different substrates on metabolite production, energy extraction and composition of gut microbiota from lean and obese subjects. PLoS One 9: e113864. 


\section{Supplemental material}

Table S1. Fold change (metabolites) in treatments 2, 3 and 4 with respect to treatment 1 (reference).

\begin{tabular}{|c|c|c|c|c|c|c|c|c|c|c|c|c|c|c|c|c|c|c|c|c|c|}
\hline \multirow{2}{*}{ Time } & \multicolumn{3}{|c|}{ acetate } & \multicolumn{1}{|c|}{ propionate } & \multicolumn{2}{c|}{$\boldsymbol{n}$-butyrate } & \multicolumn{2}{c|}{ Total SCFA } & \multicolumn{2}{|c|}{$\boldsymbol{i}$-butyrate } & \multicolumn{2}{c|}{$\boldsymbol{i}$-valerate } & \multicolumn{2}{c|}{ Total BCFA } \\
\hline & T2 & T3 & T4 & T2 & T3 & T4 & T2 & T3 & T4 & T2 & T3 & T4 & T2 & T3 & T4 & T2 & T3 & T4 & T2 & T3 & T4 \\
\hline-16 & 1.09 & 0.72 & 0.78 & 1.16 & 0.68 & 0.60 & 0.93 & 0.61 & 0.68 & 1.04 & 0.68 & 0.71 & $*$ & $*$ & $*$ & 1.10 & 0.67 & 0.66 & $*$ & $*$ & $*$ \\
\hline-14 & 0.66 & 0.40 & 0.49 & 0.86 & 0.47 & 0.46 & 0.87 & 0.59 & 0.71 & 0.74 & 0.45 & 0.54 & $*$ & $*$ & $*$ & 1.08 & 0.01 & 0.01 & 1.08 & 0.01 & 0.01 \\
\hline-8 & 0.71 & 0.79 & 0.82 & 0.71 & 0.06 & 0.09 & 0.64 & 0.09 & 0.23 & 0.69 & 0.55 & 0.60 & $*$ & $*$ & $*$ & 0.74 & 0.01 & 0.01 & 0.74 & 0.01 & 0.01 \\
\hline 0 & 0.88 & 1.23 & 1.23 & 0.82 & 0.16 & 0.23 & 0.80 & 0.50 & 0.73 & 0.84 & 0.81 & 0.89 & 0.62 & $*$ & $*$ & 0.74 & 0.18 & $*$ & 0.72 & 0.15 & $*$ \\
\hline 24 & 0.93 & 1.18 & 1.38 & 0.92 & 0.36 & 0.57 & 0.92 & 0.83 & 0.81 & 0.93 & 0.88 & 1.01 & 0.61 & 0.04 & 0.05 & 0.72 & 0.16 & 0.12 & 0.69 & 0.12 & 0.10 \\
\hline 48 & 0.91 & 1.05 & 1.22 & 1.00 & 0.53 & 0.72 & 0.99 & 1.01 & 0.93 & 0.96 & 0.92 & 1.00 & 0.65 & 0.17 & 0.24 & 0.74 & 0.33 & 0.34 & 0.72 & 0.28 & 0.31 \\
\hline 72 & 0.89 & 0.96 & 1.09 & 0.99 & 0.68 & 0.82 & 1.02 & 1.10 & 1.01 & 0.96 & 0.94 & 1.00 & 0.63 & 0.36 & 0.50 & 0.75 & 0.48 & 0.55 & 0.72 & 0.45 & 0.54 \\
\hline
\end{tabular}

*Values below the detection level

${ }^{\mathrm{a}}$ For each treatment, the ratio between each sampling time point was calculated with respect to the corresponding sampling time of the reference (i.e., t-16 treatment $2 / \mathrm{t}-16$ treatment 1 ). A value equal to 1 indicates no change; a value of $>1$ indicates an increase; and a value of $<1$ indicates a decrease of the respective metabolite.

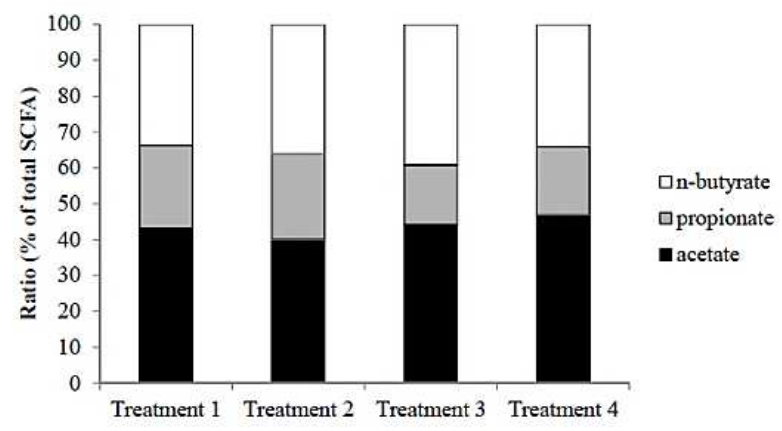

Fig. S1. Ratios of acetate, propionate and butyrate produced after 72 $\mathrm{h}$ fermentation. Samples were taken in time and analyzed by gaschromatography. The cumulative production of each individual SCFA (acetate, propionate and butyrate) and their sum (total) was calculated using the concentration from the GC analysis and volumes of the compartments of the model (lumen and dialysate). The ratio was calculated by dividing the concentration of each individual SCFA by the total. Treatment 1: feces mixed with dialysate and glycerol; Treatment 2: feces mixed with dialysate and glycerol and then frozen; Treatment 3: feces mixed with glycerol and frozen; Treatment 4: feces frozen immediately. 


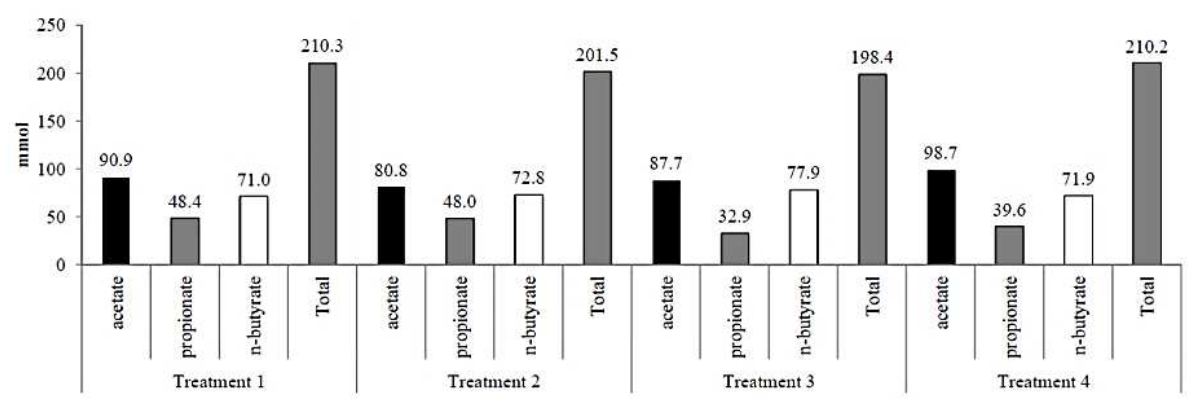

Fig. S2. Cumulative SCFA production $(t=72)$ from the fermentations. Analysis was performed as described in Fig. S1. The final cumulative amount produced after $72 \mathrm{~h}$ is plotted. Treatment 1 : feces mixed with dialysate and glycerol; Treatment 2: feces mixed with dialysate and glycerol and then frozen; Treatment 3: feces mixed with glycerol and frozen; Treatment 4: feces frozen immediately.

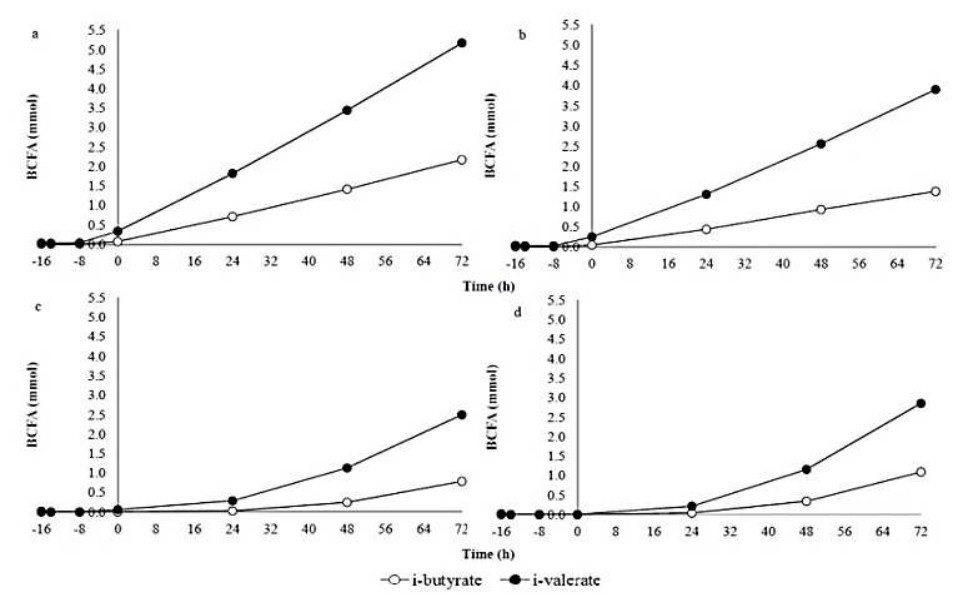

Fig. S3. Kinetics of cumulative production of BCFA during the $72 \mathrm{~h}$ experimental time. Samples were taken in time and analyzed by gaschromatography. The cumulative production of each individual BCFA (iso-butyrate and iso-valerate) and their sum (total) was calculated using the concentration from the GC analysis and volumes of the compartments of the model (lumen and dialysate). (a) Treatment 1: feces mixed with dialysate and glycerol; (b) Treatment 2: feces mixed with dialysate and glycerol and then frozen; (c) Treatment 3: feces mixed with glycerol and frozen; (d) Treatment 4: feces frozen immediately. 


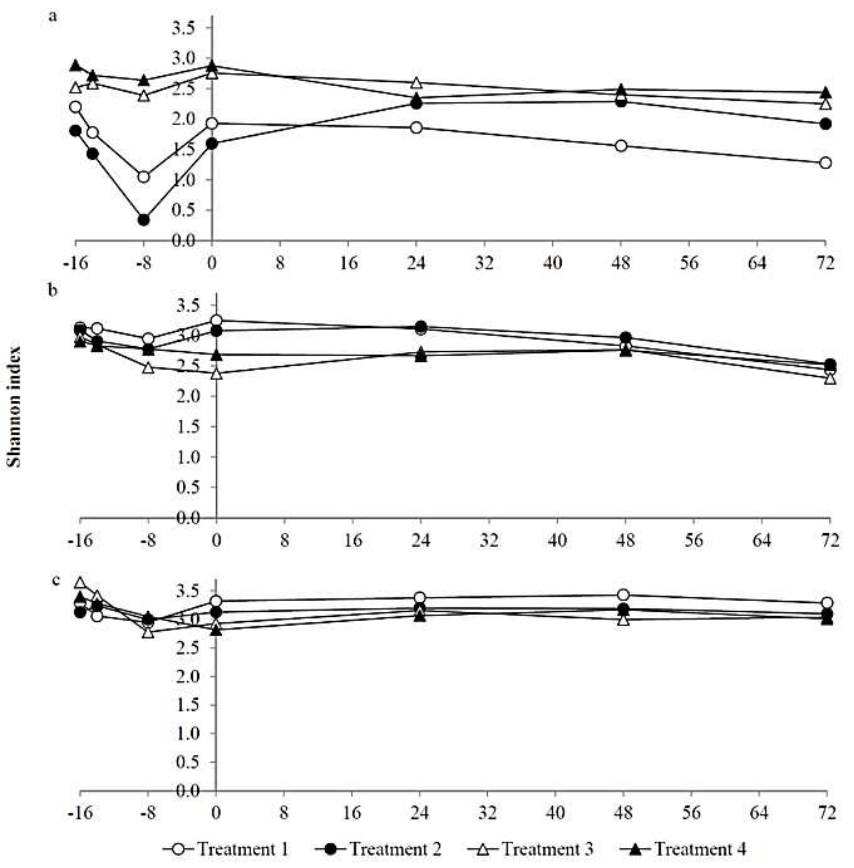

Fig. S4. Changes in Shannon index from (a) Act/Fir/Fus/Verr, (b) Bacteroidetes and (c) Proteobacteria over time. Using the data from the IS-Pro analysis, the Shannon index was calculated for the three individual groups that were analyzed. Treatment 1 : feces mixed with dialysate and glycerol; Treatment 2: feces mixed with dialysate and glycerol and then frozen; Treatment 3: feces mixed with glycerol and frozen; Treatment 4: feces frozen immediately.
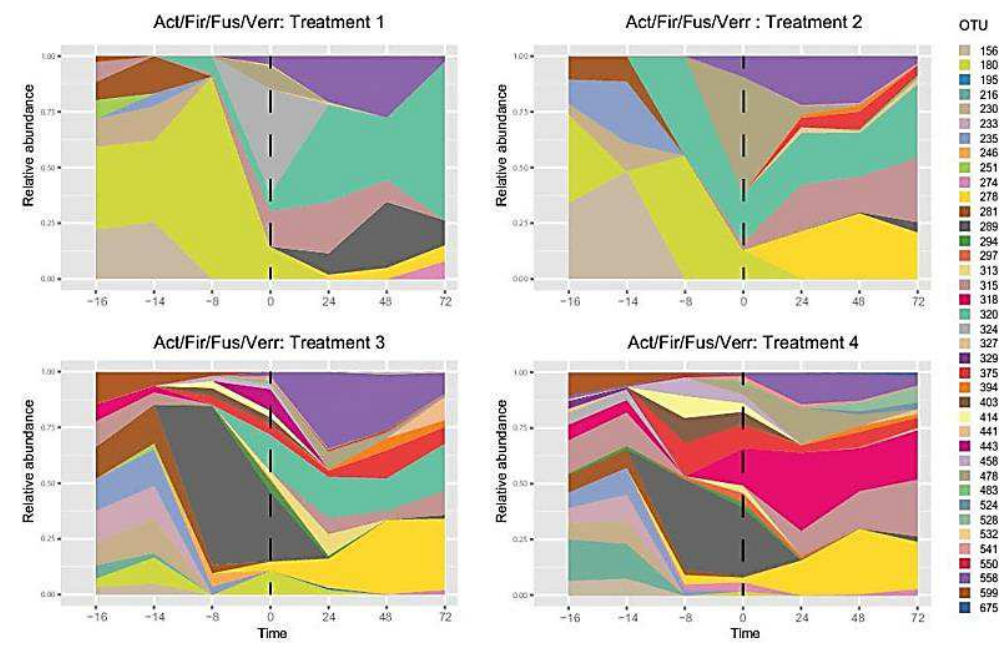

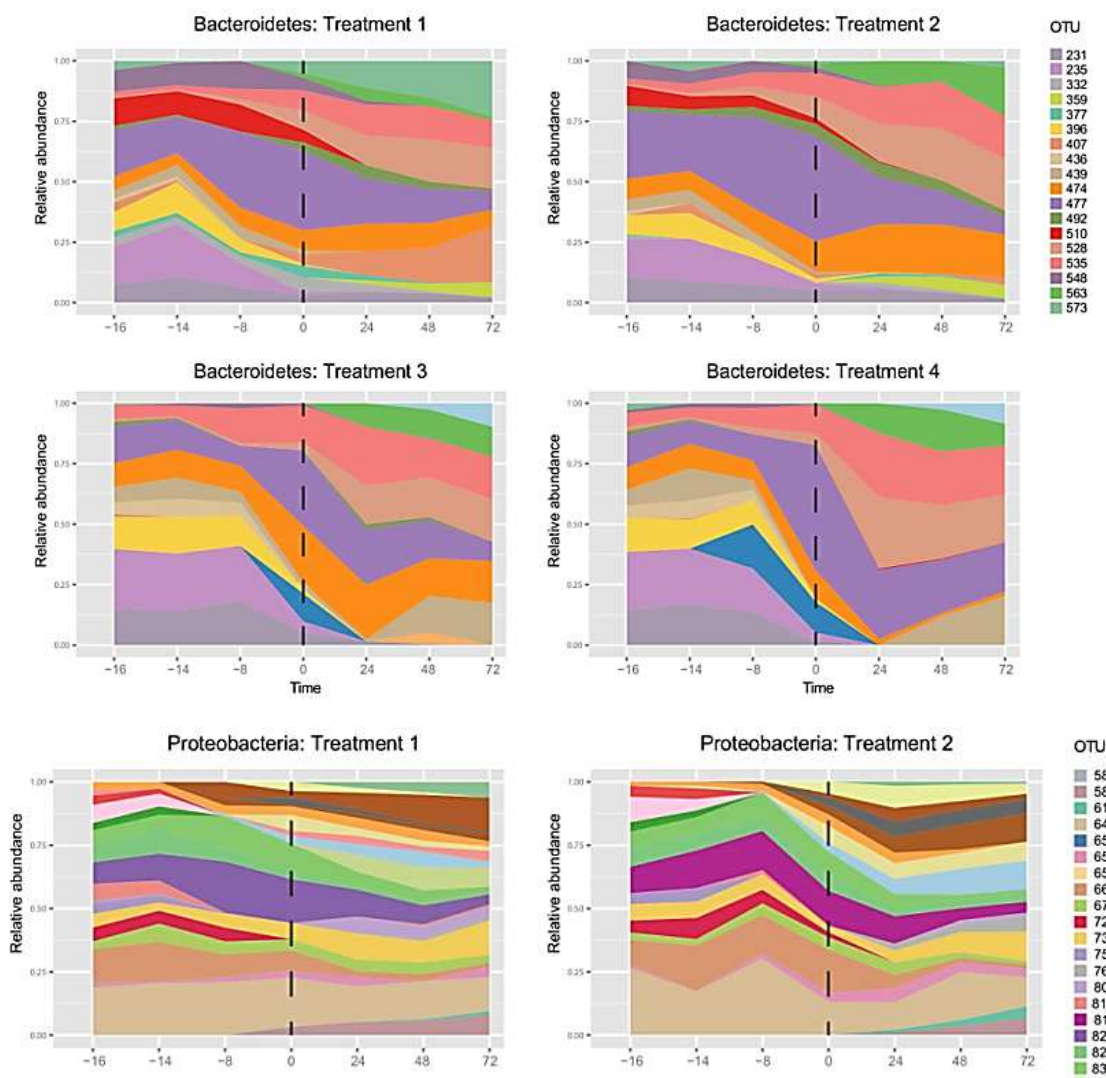

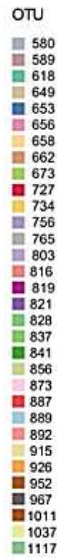
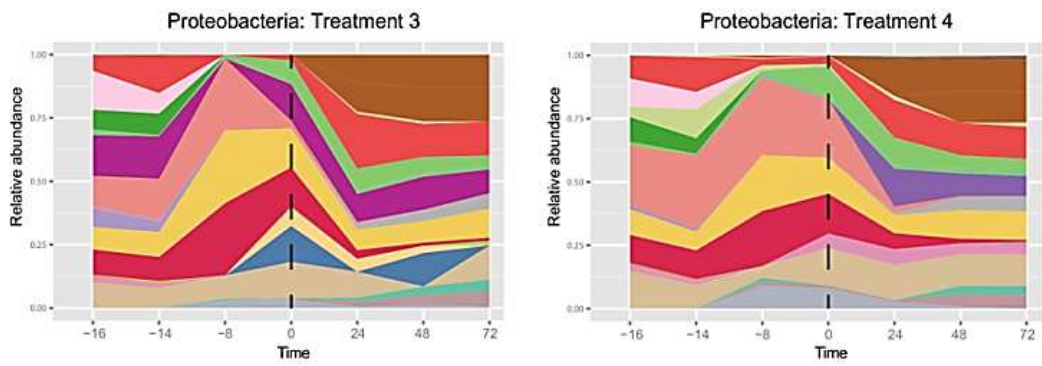

Fig. S5. Shifts of selected OTUs over time for each treatment. Changes in the abundance of peaks in the IS-Pro profiles were calculated over time. OTUs with at least a $\log _{2}$ intensity $>13$ (intensity > $8192 \mathrm{RFU}$ ) were selected and represented using a relative abundance visualization. 
Chapter 3 


\section{Chapter 4}

Seeing is believing: a repeatable, robust and reproducible in vitro simulation of the human proximal colon

Aguirre $\mathrm{M}^{*}$, Hermes $\mathrm{G}^{*}$, Ramiro-García J*, Ripken D, Kaiser D, Smidt $H$, Zoetendal E, Koenen ME, Venema K

*These authors contributed equally 


\section{Abstract}

Different criteria need to be fulfilled by in vitro gut models before they are considered valid for monitoring the effects of specific interventions/treatments on the microbiota. Important to ensure is the repeatability, robustness and reproducibility of the model. Therefore, the aim of this study was to identify these aspects and evaluate, in terms of functionality and composition, the characteristic communities developing in an in vitro gut model (TIM-2) fed either with a high carbohydrate or a high protein diet during $72 \mathrm{~h}$. To elucidate this we focused on i) determining repeatability and the robustness of the metabolic response (in terms of metabolites produced and gene transcription of a standardized fecal microbiota inoculated in the gut model and on ii) assessing the reproducibility of the compositional development of the community. Diets were compared against each other and the standard, normal diet. Both the high carbohydrate diet and the high protein diet induced a lower production of total cumulative short-chain fatty acids $(109.9 \pm 11.5 \mathrm{mmol}$ and $72.3 \pm 3.9 \mathrm{mmol}$, respectively) when compared to the normal diet $(150.4 \pm 4.09 \mathrm{mmol}, p<0.01)$. Branched-chain fatty acids and ammonia production were remarkably higher in the fermentation of the high protein diet $(5.3 \pm 0.4$ and $79.7 \pm 3.78 \mathrm{mmol}$, respectively) contrary to the high carbohydrate diet $(0.2 \pm 0.3$ and $8.12 \pm 1.95 \mathrm{mmol}$, respectively) also when compared to the normal diet $(2.87 \pm 0.57 \mathrm{mmol}, p<0.01)$. Metabolite production was reproducible and those produced from the fermentation of the high carbohydrate diet were significantly different from the high protein diet $(p<0.01)$, with the exception of propionate, and were observed to be robust through time.

It was clear that besides the reproducibility observed in the microbiota developing in each diet replicate, the largest variation in microbial composition was between the diets and not due to other technical variables. Before administrating the diet, the composition of the starting community was observed to be very similar in all 12 replicates. Such similarity was also corroborated after finding no significant difference in diversity at t0. Communities were enriched in Streptococcus and Lactococcus species. After exposing the bacteria to the different diets, distinctive microbial compositions were formed. The high carbohydrate was characterized by a high relative abundance of Subdoligranulum, Blautia, Prevotella and Bifidobacterium whilst the fermentation of the high protein diet stimulated the growth of Bacteroides, Lachnospiraceae incertae sedis, Dorea and Prevotella. The largest change in composition occurred within the first $24 \mathrm{~h}$ of the fermentation of the diets.

When moving from a general diversity/compositional richness to a functionallyrelevant gene expression analysis, the contribution of the gut microbiota in each 
diet proved to be reproducibly and robustly represented in the TIM- 2 system through time.

In conclusion, a high resolution analysis of the microbiota growing in the TIM-2 system demonstrated the repeatable, robust and reproducible development of the microbiota at both functional and compositional level. 


\section{Introduction}

The human gut microbiota consists of a complex community with a higher coding capacity than the host [1]. This densely inhabited ecosystem performs different structural, protective and metabolic functions.

Dysbiosis of human gut microbial communities has been shown to be associated to disease [2]. Therefore, many studies have been focused on investigating how to modulate the composition and metabolic activity of this ecosystem with the aim of improving host health or even to prevent or tackle serious health conditions, such as obesity.

In vivo and in vitro models have served as valuable tools to investigate fermentation in the gastro-intestinal tract and to explore the complexity of microbemicrobe interactions [2-4]. In the particular case of in vitro models, many setups have been developed in order to unravel the link between these microbes and multiple environmental components. For instance, different applications have been used to assess the bioavailability of environmental contaminants [5,6], impact of fermentation of prebiotics $[7,8]$ on the gut ecosystem, with some mechanistic studies using stable isotopes $[4,9,10]$ among others.

In vitro gut fermentation models overcome many limitations from in vivo studies which include issues of costs, volunteer compliance, ethical approval [11], difficulties associated with sampling from different regions in the gut, and their inability to determine effects based solely on microbiota activity [12]. However, different criteria need to be fulfilled by in vitro gut models before they are considered valid for monitoring the effects of specific interventions/treatments on the microbiota. It is important to ensure repeatability, robustness and reproducibility of the model since these are crucial for i) comparing between several experiments in which it is vital to have similar starting communities [12] and ii) attributing the effects of the microbial composition/activity to the applied treatment and not to the adaptation of this microbiota to the artificial environment $[4,12]$. Furthermore, the model must be capable to maintain the characteristic high diversity [2] and functional complexity of human gut microbiota.

In this study, we compositionally and functionally characterized a standardized microbial community in an in vitro model simulating the proximal colon: the TIM2 system [13]. The aim of this study was to evaluate the characteristic communities that develop in the in vitro gut model during $72 \mathrm{~h}$ when fed either a high carbohydrate or a high protein diet during $72 \mathrm{~h}$. To elucidate this we focused on i) determining the reproducibility and robustness of both the metabolic response (in terms of metabolites produced and gene transcription) of a standardized fecal 
microbiota inoculated in the gut model and on ii) assessing the reproducibility of the compositional development of such communities.

\section{Materials and methods}

\section{Microbiota}

The inoculum used for the TIM-2 experiments consisted of an active, pooled fecal microbiota prepared from 7 healthy volunteers (male: $n=3$, female: $n=4$, average age $=42 \pm 13 \mathrm{y}, \mathrm{BMI}=24 \pm 1.9 \mathrm{~kg} / \mathrm{m}^{2}$ ). We have previously shown that pooling does not result in an aberrant microbiota composition or activity [14].

Fecal samples were collected using a container kit which was maintained under anaerobiosis by using anaerobic packs (AnaeroGen ${ }^{\mathrm{TM}}$, Oxoid, Cambridge, UK). Samples were homogenized in an anaerobic cabinet $\left(80 \% \mathrm{~N}_{2}, 10 \% \mathrm{CO}_{2}, 10 \% \mathrm{H}_{2}\right)$ and were used to inoculate a fed-batch fermentor simulating the human 'cecum' conditions as described earlier [13]. In short, $670 \mathrm{ml}$ food was inoculated with $80 \mathrm{~g}$ of pooled stools. The incubation lasted $44 \mathrm{~h}$ at $37^{\circ} \mathrm{C}$ and was constantly flushed with gaseous nitrogen in order to guarantee anaerobic conditions. In addition, $1250 \mathrm{ml}$ of food was supplied in fed-batch mode during the incubation time. The resulting slurry was aliquoted and snap-frozen in liquid nitrogen $\left(-196^{\circ} \mathrm{C}\right)$. This microbiota was stored at $-80^{\circ} \mathrm{C}$ before inoculation in TIM-2.

\section{Fermentation media}

During the period in which the microbiota was left to adapt to the conditions of the fermentation model (16h), all TIM-2 units were fed with simulated ileal efflux medium (SIEM) as described by Maathuis et al. [15]. After this adaptation period the units were fed with normal, high carbohydrate $(\mathrm{CHO})$ and high protein diets (1:1, 10:1 and 1:10 (CHO: protein) diets) as described next.

Normal diet; 1:1 (CHO: protein)

This feeding containing the basal medium (normal preparation of SIEM) was used as a control. This preparation is always used for fermentation experiments in TIM2 and simulates the average non-digestible carbohydrates consumed in a normal Western diet [13].

High CHO diet; 10:1 (CHO: protein)

The preparation of SIEM was modified by diluting the concentration of TBCO 10 times (mixture of Tween 80, bacto-peptone, caseine and ox-bile) while keeping the proportions of the rest of the ingredients in the basal medium the same. 
High protein diet; 1:10 (CHO: protein)

The content of carbohydrates in the basal medium (pectin, xylan, arabinogalactan, amylopectin and starch) was diluted 10 times. The quantities of the rest of the ingredients were the same as in the basal medium.

\section{TIM-2 fermentation experiments}

The study was performed in two different experimental weeks in which six units were run in parallel per week. The TIM-2 system was flushed with $\mathrm{N}_{2}$ prior to the introduction of the inoculum and it was maintained under this condition at $37^{\circ} \mathrm{C}$ for 96h. The $\mathrm{pH}$ was kept at or above 5.8 by automatic titration with $\mathrm{NaOH}(2 \mathrm{M})$. Furthermore, the atmosphere in the system was progressively enriched by gases resulting from the fermentation process by the microbiota, contributing to the anaerobiosis in the system.

A $30 \mathrm{ml}$ portion of fecal homogenate was used to inoculate the separate units for each experiment. Each unit was then filled to $120 \mathrm{ml}$ with $90 \mathrm{ml}$ of dialysate [15]. Right after the inoculation, the microbiota was left to adapt (16h) to the new environment. During this period, the basal medium preparation (SIEM) was gradually introduced into the system in a total volume of $40 \mathrm{ml}$. After adaptation, the community was deprived from any medium for $2 \mathrm{~h}$ (starvation) in order to maximize the use of the tested diets by the microbiota [16]. After the starvation period (at t0), the test diets were added. A volume of $180 \mathrm{ml}$ of the different diets and control was administrated over the $72 \mathrm{~h}$ of the test period at a rate of $2.5 \mathrm{ml} / \mathrm{h}$.

Throughout the experiment, luminal content was maintained at a level of approximately $120 \mathrm{ml}$ in each unit by a level sensor (liquiphant FTL20-0025, Endress+Hauser). In order to remove fermentation products and water from the lumen, a dialysis system (described in detail by van Nuenen et al. [17]) consisting of a semi-permeable hollow membrane ran through the lumen. For all the experiments, the speed of the dialysis was set at $1.5 \mathrm{ml} / \mathrm{min}$.

After 24 and $48 \mathrm{~h}$ of starting with the addition of the test diets, $25 \mathrm{ml}$ of lumen sample was removed from the system to mimic the transit of material coming from the proximal and reaching the distal colon [15].

Luminal and dialysate sampling was performed at 0,24, 48 and $72 \mathrm{~h}$. Samples were stored $\left(-80^{\circ} \mathrm{C}\right)$ until analysis.

\section{Metabolite production}

SCFA (acetate, propionate and butyrate) and BCFA (iso-butyrate and iso-valerate) analyses were performed as described by van Nuenen et al.[17]. In brief, samples were centrifuged $(\sim 12.000 \mathrm{~g}, 5 \mathrm{~min})$ and to the clear supernatant a mixture of formic acid (20\%), methanol and 2-ethyl butyric acid (internal standard, $2 \mathrm{mg} / \mathrm{ml}$ in 
methanol) was added. A $3 \mu \mathrm{l}$ sample with a split ratio of 75.0 was injected on a GC-column (ZB-5HT inferno, ID $0.52 \mathrm{~mm}$, film thickness $0.10 \mathrm{um}$; Zebron; phenomenex, USA) in a Shimadzu GC-2014 gas chromatograph. Ammonia analyses were performed by Bio-aNAlytiX (Mook, The Netherlands) [18].

Statistical analysis

The experiments were performed in series of four per diet $(n=4)$. Results are presented as mean \pm SD. Statistical analysis was performed using the SAS statistical software package (SAS version 9: SAS Institute, Cary, NC). All metabolites were compared with a mixed analysis of variance (ANOVA) model. Data were visually checked on normality and on constant variance of residuals by plots of residuals vs. corresponding predicted values. If data was not normally distributed, log transformation was applied for further analysis of the data as was the case for all metabolites tested. No outliers were detected. The ANOVA model considered diet (normal, high $\mathrm{CHO}$ and high protein) and time (t0, t24, t48, t72) as fixed factors. The factor replicate, which is the number of experiments performed was included in the model as a random factor. The Tukey-Kramer post hoc test was used to correct for multiple comparisons. The concentration of all metabolites was artificially set to zero at t0. To avoid unnecessary repetition, this is not indicated further in the text or graphs in the results section. Data were considered significant at $p<0.05$.

\section{Gut microbiota profiling}

Total DNA extraction

For the extraction of genomic DNA, $250 \mu 1$ of luminal sample was mixed with 250 $\mu \mathrm{l}$ of TE buffer (Tris-HCl pH 7.6, EDTA pH 8.0), $50 \mu 1$ 10\% (v/v) SDS (Ambion, Austin, TX, USA) and $500 \mu \mathrm{l}$ acid-phenol (Phenol:Water $(3.75: 1 \mathrm{v} / \mathrm{v}) ; \mathrm{pH}=$ 4.45-5.68; Invitrogen, Carlsbad, CA, USA). Samples were treated 3x in a FastPrep (Precellys 24, Bertin Technologies, USA) at speed 5.5 for $45 \mathrm{~s}$ and later centrifuged at $13.400 \mathrm{~g}$ for $15 \mathrm{~min}$ at $4{ }^{\circ} \mathrm{C}$. Nucleic acids were subsequently purified by extraction with Maxwell MDx (Promega, Madison, USA) following the "16 Tissue LEV Total RNA" protocol according to manufacturer's instruction.

Primers

Primer pairs 515F (5'-GTGCCAGCMGCCGCGGTAA) - 806R (5'GGACTACHVGGGTWTCTAAT) and BSF784F (5'-RGGATTAGATACCC) 1064R (5'-CGACRRCCATGCANCACCT) have been previously reported for amplification of the V4 [19] and V5- V6 [20] regions of the bacterial 16S rRNA gene, respectively. They were selected based on 1) experimental validation, 2) 
taxonomic coverage of the relevant ecosystem adherence to specific rules associated with the sequencing platform, such as a maximum amplicon size of $<500 \mathrm{nt}$. Unless noted otherwise all primers were ordered at Biolegio (Nijmegen, Netherlands).

\section{Barcoding strategy}

To achieve optimal sample throughput and phylogenetic depth, 70 primers containing a custom designed 8nt barcode were developed to combine with Illumina barcodes. Each set of 70 barcoded samples are referred to as "library". Low diversity samples, such as $16 \mathrm{~S}$ rRNA gene amplicons, can lead to problems with base calling due to overexposure of fluorescent labels. Therefore, the set of 70 barcodes was specifically designed to possess an equal base distribution over their complete length. Additionally, to avoid differential amplification, a two-base "linker" sequence that is not complementary to any 16SrRNA sequence at the corresponding position was inserted between the primer and barcode. The resulting set of 70 barcoded primers was checked for avoidance of secondary structure formation within or between primers (i.e., primer-dimers) or between barcodes and primers, using PrimerProspector [21].

\section{Barcoded PCR}

To ensure reproducible results, 2 variable regions (V4 and V5-V6) of the $16 \mathrm{~S}$ rRNA gene were used. Each sample was amplified in duplicate using Phusion hot start II high fidelity polymerase (Thermo fisher scientific AG, Reinach, Zwitserland), checked for correct size and concentration on a $1 \%$ agarose gel and subsequently combined and column-purified with the High pure PCR cleanup micro kit (Roche diagnostics, Mannheim, Germany). PCR reactions were performed in $50 \mu \mathrm{l}$ reactions according to the manufacturer's instruction (Fisher Scientific, Landsmeer, the Netherlands). PCR reactions contained $36.5 \mu \mathrm{l}$ nucleotide free water (Promega, Madison, USA), $0.5 \mu \mathrm{L}$ of $2 \mathrm{U} / \mu$ l polymerase, 10 $\mu \mathrm{l}$ of $5 \mathrm{x} 10 \mu \mathrm{l}$ buffer green HF (5x), $1 \mu \mathrm{l}$ of $10 \mu \mathrm{M}$ stock solutions of each of the forward (515F) and reverse (806R) primers, $1 \mu \mathrm{l} 10 \mathrm{mM}$ dNTPs (Promega, Madison, USA) and $1 \mu \mathrm{L}$ template DNA ( $10^{3} \mathrm{x}$ diluted $200 \mathrm{ng} / \mathrm{ul}$ stock). Reactions were held at $98{ }^{\circ} \mathrm{C}$ for $30 \mathrm{~s}$ and amplification proceeding for 25 cycles at $98{ }^{\circ} \mathrm{C}$ for $10 \mathrm{~s}, 52{ }^{\circ} \mathrm{C}$ for $10 \mathrm{~s}, 72{ }^{\circ} \mathrm{C}$ for $10 \mathrm{~s}$ and a final extension of $7 \mathrm{~min}$ at $72{ }^{\circ} \mathrm{C}$. Purified amplicons were quantified using Qubit. For primer pair BSF784F-1064R the thermal cycling conditions were identical to those detailed above except that the annealing temperature was $42{ }^{\circ} \mathrm{C}$. A composite sample for sequencing was created by combining equimolar amounts of amplicons from the individual samples, followed by purification and concentration using magnetic beads to remove any 
remaining contaminants. The resulting libraries were sent to GATC Biotech AG (Konstanz, Germany) for sequencing on an Illumina Hiseq2000 instrument.

Unless specified, the data presented the combined information of both regions. The average number of reads per sample was $166311 \pm 131574$.

Analysis of community structure

Raw 16S rRNA gene amplicon data was analyzed using NG-tax (Ramiro-García et $a l$, submitted) using default settings as described elsewhere. Statistical analysis and visualization were done with QIIME suite of software v 1.9 [22] and R version 3.1.3. A heatmap showing the significantly different taxa (genus level) between the diets was made calculating the difference of relative abundance means using a Kruskal wallis test.

Total RNA extraction and rRNA removal

Total RNA was extracted from the luminal samples following the Macaloid-based RNA isolation protocol [23] which incorporated the use of a phase lock gel ${ }^{\mathrm{TM}}$ (GLP $\left.{ }^{\mathrm{TM}}\right)$ in order to effectively isolate the nucleic acid-containing phase from the sample as previously explained [24]. RNeasy mini kit (QIAGEN, CA, USA) was used for RNA purification and included the use of an on-column DNAse I (Roche, Germany) treatment [23] to remove DNA from the samples. RNA quantity and quality were assessed using a NanoDrop 2000/2000C spectrophotometer (Thermo Scientific, USA), Experion RNA Stdsens analysis kit (Biorad Laboratories Inc., USA) and plotting 23S/16S rRNA ratios (Experion ${ }^{\mathrm{TM}}$ Software V. 3.0, Bio-Rad, USA).

Extracted RNA was enriched using the Ribo-Zero ${ }^{\mathrm{TM}}$ rRNA removal kit (Epicentre, Madison, USA) according to the manufacturer's instructions. The rRNA-depleted sample was purified by precipitation with ethanol. In brief, the volume of each RNA sample was adjusted to $180 \mu 1$ using RNase-Free water and it was subsequently mixed with $18 \mu \mathrm{l}$ of $3 \mathrm{M}$ sodium acetate, $2 \mu \mathrm{l}$ of glycogen $(10 \mathrm{mg} / \mathrm{ml})$ and 3 volumes of ice-cold $100 \%$ ethanol. Tubes were stored overnight at $-20{ }^{\circ} \mathrm{C}$ and were subsequently centrifuged at $>10.000 \mathrm{~g}$ at $4{ }^{\circ} \mathrm{C}$ for $30 \mathrm{~min}$. The supernatant was carefully removed and the pellet was washed twice with $600 \mu \mathrm{l}$ ice-cold $70 \%$ ethanol. The pellet was allowed to air dry at room temperature for 5 min. Finally, the pellet was dissolved in $10 \mu \mathrm{l}$ of RNase-Free water. The quality of the treated RNA was assessed using a NanoDrop 2000/2000C spectrophotometer (Thermo Scientific, USA) and Experion RNA Stdsens analysis kit (Biorad Laboratories Inc., USA).

The Ribo-Zero ${ }^{\mathrm{TM}}$ treated RNA was used for the ScriptSeq ${ }^{\mathrm{TM}}$ (Epicentre, Madison, USA) library preparation according to the manufacturer's instructions. For the 
purification steps we used the HighPrep PCR kit (MagBio Genomics, Inc., US/Canada), according to the manufacturer's instructions. The samples were sent for sequencing at GATC-Biotech (Konstanz, Germany).

\section{Metatranscriptomic analysis}

Despite the mRNA enrichment step described above, rRNA depletions are generally incomplete, therefore to remove the remaining rRNA sequences form the RNA-seq datasets the software SortMeRNA [25] and the protocol described in Leimena et al. [26] was employed. The pipeline described in Davids et al. [27] was used to assembly the unfiltered reads (potential mRNA reads) into contigs, predict ORFs (Open Reading Frame) and annotate KEGG functions. Differential expression analyses of the KEGG functions were performed with the R package edgeR [28].

\section{Ethics Statement}

Studies using fecal donations from healthy volunteers do not require medical ethical committee approval in The Netherlands since they are considered as noninvasive. Nevertheless, all participants provided informed consent prior to the start of the study.

\section{Results}

$p H$

The luminal $\mathrm{pH}$ in the TIM-2 system was maintained at 5.8. Therefore, it was not possible to observe any differences in acidification among the interventions. However, by plotting the consumption of $\mathrm{NaOH}$ (used to titrate the $\mathrm{pH}$ ) as a proxy for the produced acids in the lumen, it was possible to observe a significantly higher alkaline consumption (reflecting higher acid production) for the high $\mathrm{CHO}$ diet $(p<0.01$ at $\mathrm{t} 72$ ), and a significant lower consumption for the high protein diet ( $p<0.01$ for t24, 48 and 72) compared to the normal diet (Fig. 1, Table S1). In addition, both high $\mathrm{CHO}$ and high protein diets were significantly different from each other ( $p<0.01$ from t24 to t72). Interestingly, there was no statistically significant difference in the alkali consumed after the adaptation period at t0 among the three diets. 


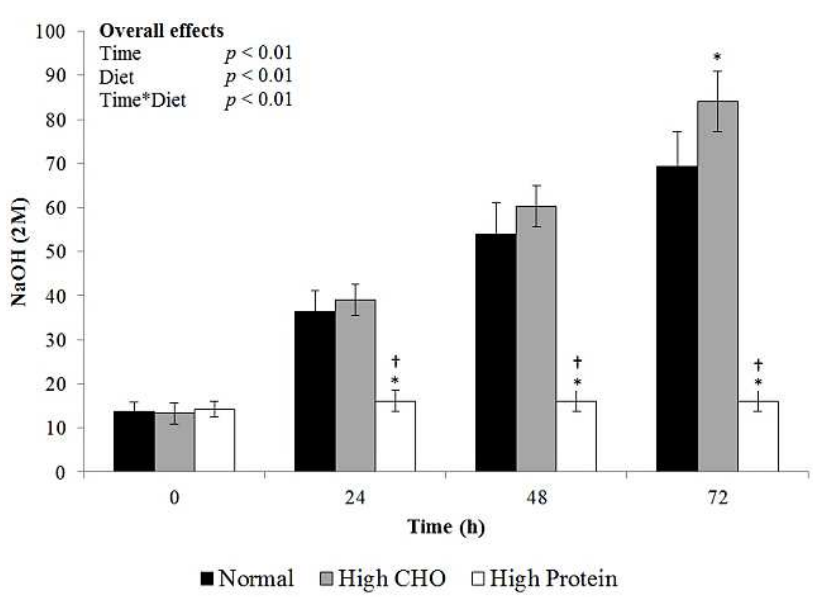

Fig. 1. Cumulative consumption of $\mathrm{NaOH}(2 \mathrm{M})$ to titrate the system after the adaptation period (bars at $\mathrm{t} 0$ ) and during the $72 \mathrm{~h}$ fermentation. The different diets correspond to: normal: 1:1 (control or basal SIEM medium); high CHO: 10:1 and high protein 1:10 (CHO: protein) diets. Key: ${ }^{*} p<0.01$ specific time point is significantly different compared to the control diet; $\dagger p<0.01$ specific time point is significantly different compared to the high CHO diet.

\section{Microbiota activity}

\section{SCFA}

Table 1 shows the total amount of SCFA (sum of acetate, propionate and butyrate) produced after $72 \mathrm{~h}$ of fermentation. The fermentation of the high $\mathrm{CHO}$ diet resulted in higher total cumulative SCFA $(109.9 \pm 11.5 \mathrm{mmol})$ compared to the high protein diet which produced the lowest amount $(72.3 \pm 3.9 \mathrm{mmol})$ although both were lower than the control $(150.4 \pm 4.09 \mathrm{mmol} ; p<0.01)$.

Table 1. Total average (+/- SD) amount of SCFA (sum of acetate, propionate and $n$-butyrate; mmol) produced in the TIM-2 system during the $72 \mathrm{~h}$ fermentation experiments with the three diets (normal, high $\mathrm{CHO}$ and high protein) ${ }^{1}$.

\begin{tabular}{|l|c|c|c|c|}
\hline \multirow{2}{*}{ Diet } & \multicolumn{4}{|c|}{ Total SCFA (mmol) } \\
\cline { 2 - 5 } & $\mathrm{t} 0$ & $\mathrm{t} 24$ & $\mathrm{t} 48$ & $\mathrm{t} 72$ \\
\hline Normal & 0 & $41.8 \pm 3.12$ & $95.0 \pm 2.10$ & $150.4 \pm 4.09$ \\
\hline High CHO & 0 & $38.3 \pm 4.88$ & $72.3 \pm 8.37^{*}$ & $109.9 \pm 11.5^{*}$ \\
\hline High protein & 0 & $22.9 \pm 2.29^{* \dagger}$ & $46.8 \pm 3.44^{* \dagger}$ & $72.3 \pm 3.85^{* \dagger}$ \\
\hline
\end{tabular}


${ }^{1}$ Key: Values were artificially set to zero at the start of feeding the different test diets (t0) $* p<0.01$ specific time point is significantly different compared to the normal diet; $\uparrow p<0.01$ specific time point is significantly different compared to the high $\mathrm{CHO}$ diet. The different diets correspond to: normal: 1:1 (control or basal SIEM medium); high CHO: 10:1 and high protein 1:10 (CHO: protein) diets.

As observed for the total SCFA (Table 1), the total cumulative production of the individual SCFA acetate, propionate and $n$-butyrate were higher with the normal diet when compared to the other diets. Both high $\mathrm{CHO}$ and high protein diets showed significant differences in the total cumulative amounts of acetate and $n$ butyrate compared to the normal diet $(p<0.01$ at t72) (Fig. 2). There was no significant difference in the propionate production over time among the diet high in $\mathrm{CHO}$ and the diet high in protein.

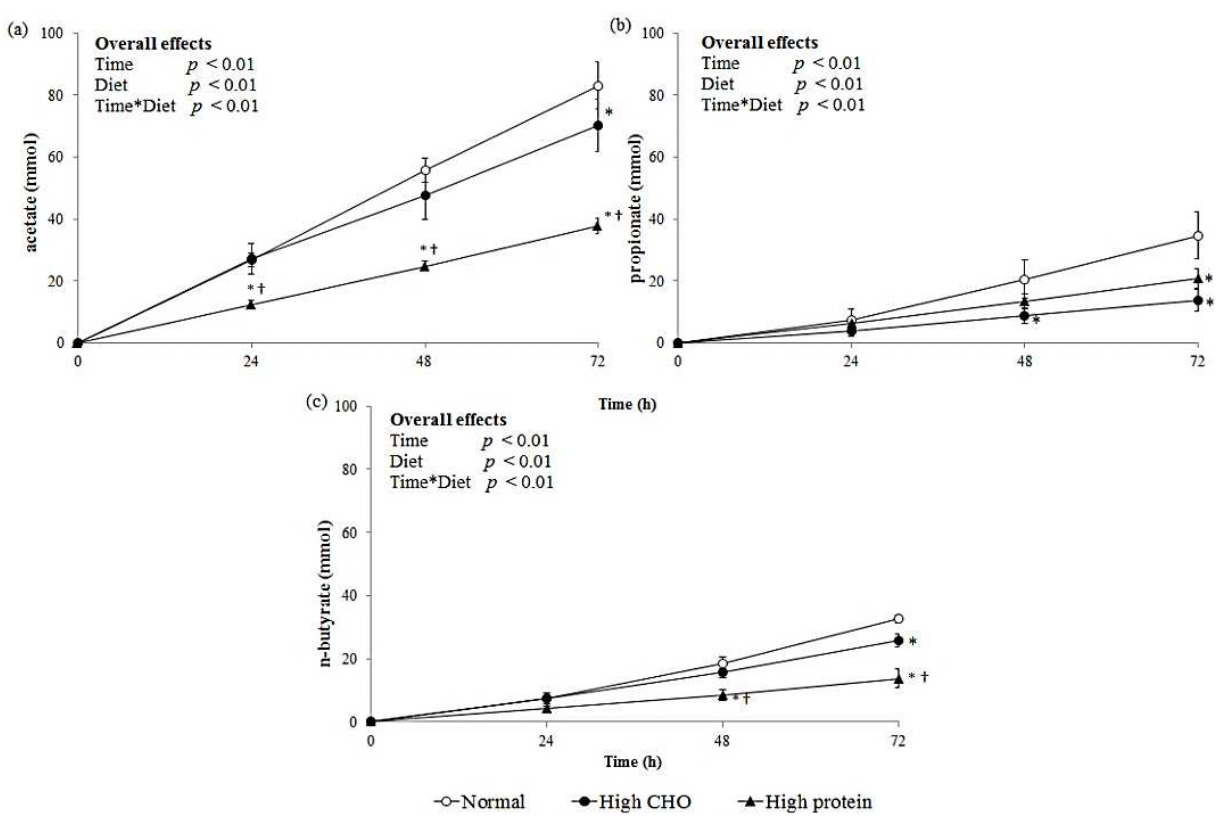

Fig. 2. Average (+/- SD) kinetics of cumulative SCFA production (mmol) of (a) acetate, (b) propionate (c) and $n$-butyrate in the experiments. Values were artificially set to zero at the start of feeding the different test diets $(\mathrm{t} 0)$. The different diets correspond to: normal: 1:1 (control or basal SIEM medium); high CHO: 10:1 and high protein 1:10 (CHO: protein) diets. Key: ${ }^{*} p<0.01$ specific time point is significantly different compared to the normal diet; $\dagger p<$ 
0.01 specific time point is significantly different compared to the high $\mathrm{CHO}$ diet.

BCFA and ammonia

Table 2 shows that after $72 \mathrm{~h}$ of fermentation of the high $\mathrm{CHO}$ diet, lower amounts of total cumulative BCFA $(0.23 \pm 0.29 \mathrm{mmol})$ were produced whilst the fermentation of the high protein diet resulted in a higher production $(5.33 \pm 0.36$ mmol) when compared to the normal diet $(2.87 \pm 0.57 \mathrm{mmol} ; p<0.01)$.

Table 2. Total average (+/- SD) BCFA (sum of iso-butyrate and isovalerate; mmol) produced in the TIM-2 system during the $72 \mathrm{~h}$ fermentation experiments with the three diets (normal, high $\mathrm{CHO}$ and high protein) ${ }^{1}$.

\begin{tabular}{|l|c|c|c|c|}
\hline \multirow{2}{*}{ Diet } & \multicolumn{4}{|c|}{ Total BCFA (mmol ) } \\
\cline { 2 - 5 } & $\mathrm{t} 0$ & $\mathrm{t} 24$ & $\mathrm{t} 48$ & $\mathrm{t} 72$ \\
\hline Normal & 0 & $0.45 \pm 0.32$ & $1.32 \pm 0.54$ & $2.87 \pm 0.57$ \\
\hline High CHO & 0 & $0.00 \pm 0$ & $0.02 \pm 0.02^{*}$ & $0.23 \pm 0.29^{*}$ \\
\hline High protein & 0 & $0.89 \pm 0.33^{\dagger}$ & $2.66 \pm 0.29^{* \dagger}$ & $5.33 \pm 0.36^{* \dagger}$ \\
\hline
\end{tabular}

${ }^{1}$ Key: Values were artificially set to zero at the start of feeding the different test diets $(\mathrm{t} 0) * p<0.01$ specific time point is significantly different compared to the normal diet; $\uparrow p<0.01$ specific time point is significantly different compared to the high $\mathrm{CHO}$ diet. The different diets correspond to: normal: 1:1 (control or basal SIEM medium); high CHO: 10:1 and high protein 1:10 (CHO: protein) diets.

The cumulative iso-valerate production was higher when compared to iso-butyrate in the experiments with the high protein diet (Fig. 3). As expected, both metabolites were produced in significantly higher amounts with this diet when compared to the others. The same was observed for ammonia amounts (Fig. 4). The production of putrefactive metabolites was remarkably lower in the experiments with the high $\mathrm{CHO} \operatorname{diet}(p<0.01$; Figs. 3 and 4$)$. 


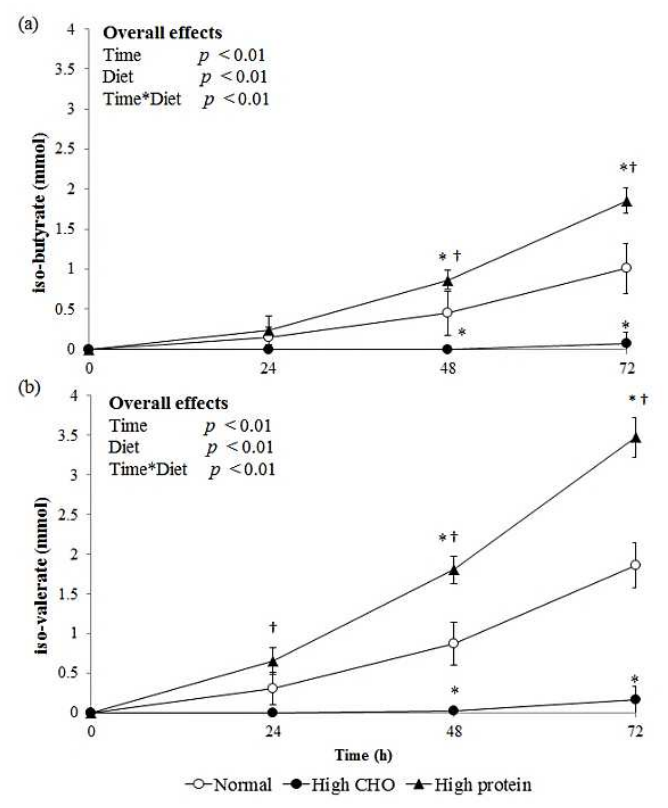

Fig. 3. Average (+/- SD) kinetics of cumulative BCFA production (mmol) in the experiments. (a) iso-butyrate, and (b) iso-valerate. Values were artificially set to zero at the start of feeding the different test diets ( $\mathrm{t} 0$ ). The different diets correspond to: normal: 1:1 (control or basal SIEM medium); high CHO: 10:1 and high protein 1:10 (CHO: protein) diets. Key: ${ }^{*} p<0.01$ specific time point is significantly different compared to the normal diet; $\uparrow p<0.01$ specific time point is significantly different compared to the high CHO diet.

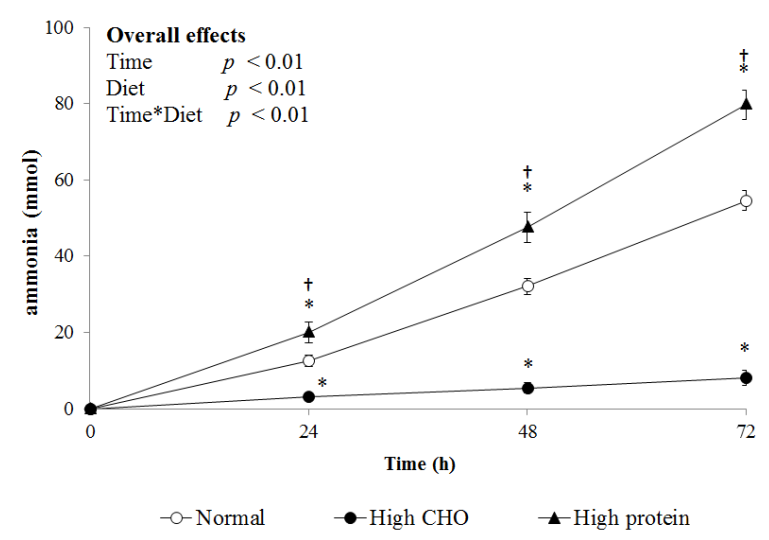

Fig. 4. Average (+/- SD) kinetics of cumulative ammonia production (mmol) in the experiments. Values were artificially set to zero at the start of feeding the different test diets ( $\mathrm{t} 0)$. The different diets 
correspond to: normal: 1:1 (control or basal SIEM medium); high CHO: 10:1 and high protein 1:10 (CHO: protein) diets. Key: ${ }^{*} p<$ 0.01 specific time point is significantly different compared to the normal diet; $\uparrow p<0.01$ specific time point is significantly different compared to the high $\mathrm{CHO}$ diet.

\section{Microbiota profiling}

To ensure reproducible results with 16S rRNA gene amplicons sequencing, two variable regions were amplified and duplicate technological replicates were performed. Finally, the data was sequenced at two separate occasions. All datasets were found to be in complete agreement.

Population dynamics

After inoculation and during the stabilization phase the phylogenetic diversity [29] dropped (Fig. S1). After the stabilization phase, each diet formed its specific microbiological composition (Fig. 5). The unweighted Unifrac showed the largest separation between the diets and t0, which indicates not only changes in the abundance, but also which OTUs were above the cutoff. Since unweighted Unifrac puts large emphasis on this (arbitrary) cutoff and is more sensitive to sequencing error, we used weighted Unifrac for the remainder of the analysis. However, both metrics were in complete concordance (Fig. 5).

The compositional similarity within all TIM-2 units at the start (t0) of the experiments was corroborated after finding no significant difference in relative abundance at genus level between units (FDR p>0.05) and diversity.

After the administration of the different diets, the composition of the microbiota started to deviate in time, in a specific way for each diet as shown with the weighted Unifrac distances (Fig 5). Similarly, the diversity increased through the $72 \mathrm{~h}$ fermentation of the diets (Fig. 6), suggesting a continued change in composition, although after the first $24 \mathrm{~h}$ the change was observed to slow down.

Despite that there were minor differences in composition within the TIM-2 units, the diet effect was clearly visible (Fig. 7) and within diet Unifrac distances were smaller than between diet distances (Fig.8; $\mathrm{p}<0.001$ ). Overall, the reproducibility of the system was clear from the weighted Unifrac distance analysis (Fig. 8): i) the distances between experimental days and runs were significantly shorter than between "random variables" (all variables combined), ii) the distances "within the diets" are shorter than the "between diet" distances, and iii) the distances between the PCR replicates were significantly smaller than the biological findings. All in all, from Fig.8 it was clear that besides the reproducibility observed in the 
microbiota developing in each diet replicate, the largest variation in microbial composition was between the diets and not due to other technical variables.
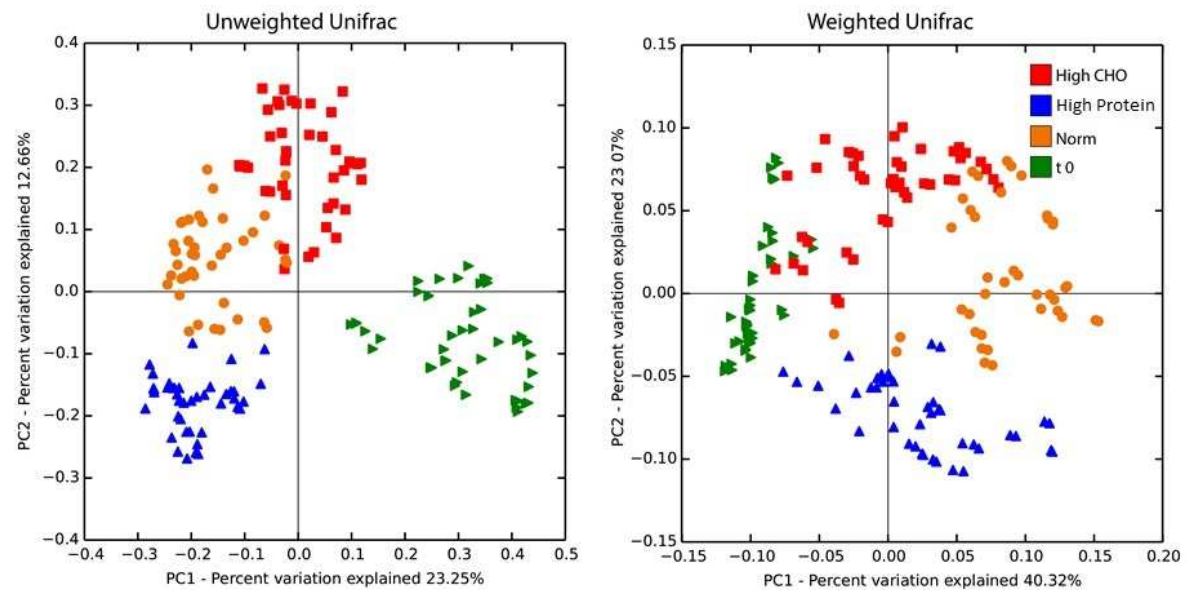

Fig. 5. PCoA plots with Unweighted (left) and Weighted (right) Unifrac distances.

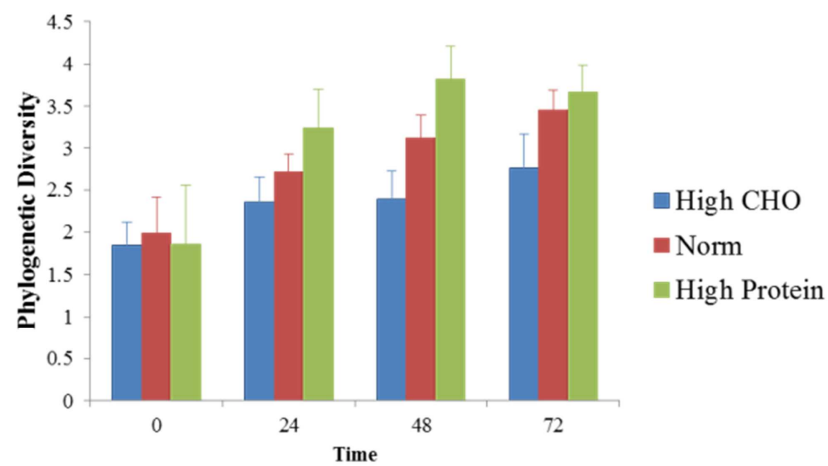

Fig. 6. Phylogenetic diversity of the diets at each time point 


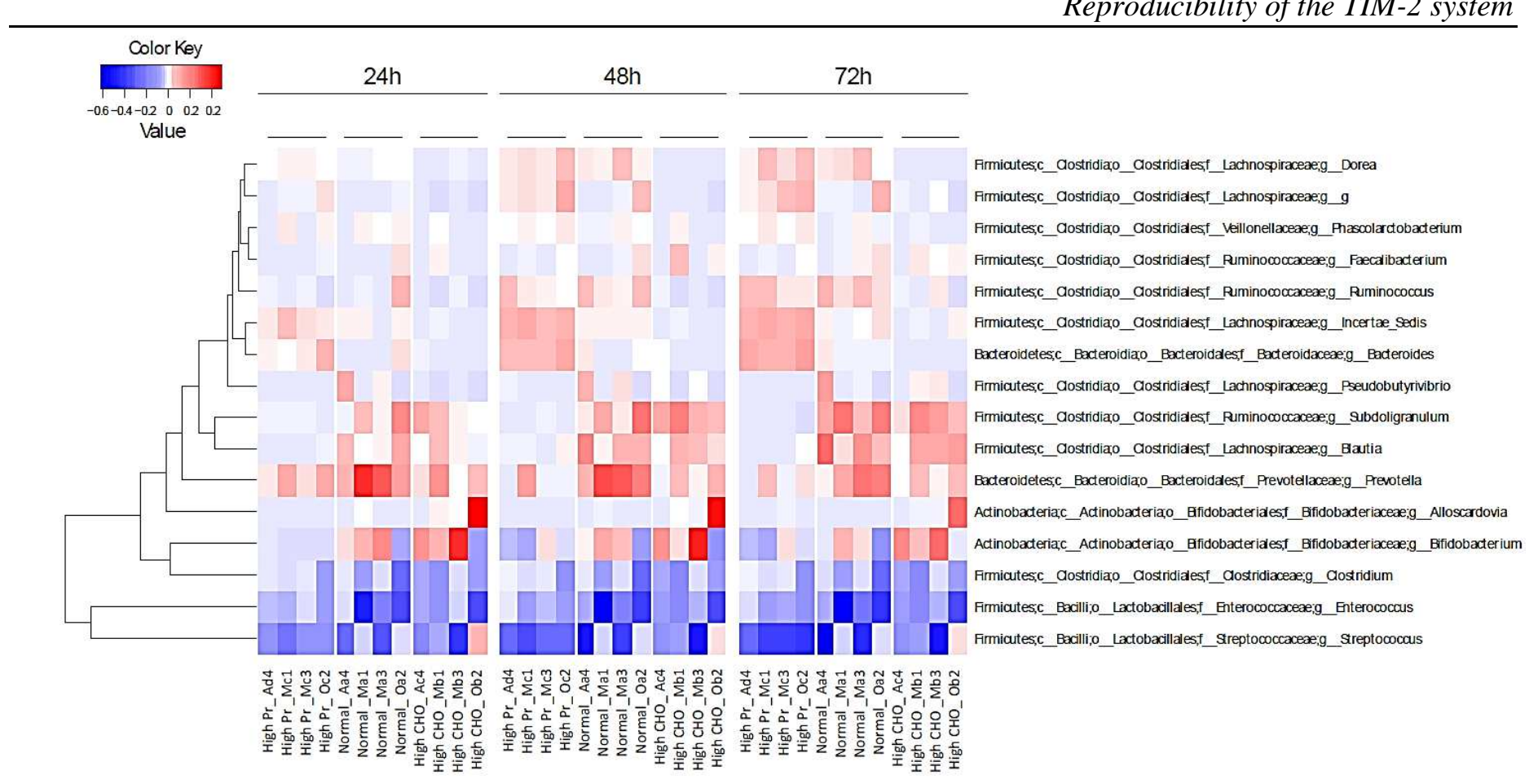

Fig 7. Heatmap of significantly different taxa at genus level between the diets (difference of relative abundance means using a Kruskal wallis test). Colors show relative abundance compared to the same unit at t0. Blue is a decrease, red increase. 


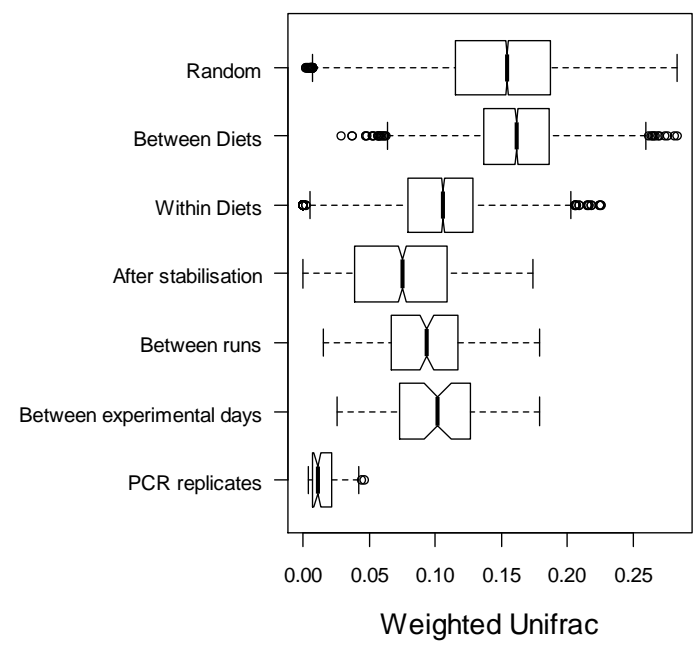

Fig. 8. Boxplots showing weighted Unifrac distances for different categories (notches not overlapping $=$ significant)

Development of the microbiota fermenting the diets in the TIM-2 units

As mentioned earlier, the composition of the microbial community before initiating the diet administration was observed to be reproducible in all replicates. The composition at t0 was characterized by high relative abundances of mainly facultative anaerobes, such as Streptococcus, and Lactococcus.

The fermentation of the different diets showed a distinct microbial composition, however some patterns were clearly visible within all diets and units: Clostridium, Enterococcus and Streptococcus decreased and Prevotella increased (Fig. 7; except for $\mathrm{Ob}$, which shows a slightly deviant pattern compared to the other units with the same diet). At 24 significant compositional differences were observed between the diets, mainly in Prevotella, Bacteroides and Lachnospiraceae incertae sedis abundances. The bacteria that increased after the $24 \mathrm{~h}$ fermentation of the high $\mathrm{CHO}$ and normal diets were Subdoligranulum, Blautia, Prevotella and Bifidobacterium. Bacteroides, Lachnospiraceae incertae sedis, Dorea, and Prevotella where highly abundant in the microbiota fermenting the high protein diet after $24 \mathrm{~h}$. At $48 \mathrm{~h}$, these bacteria were seen to increase with the exception of Prevotella which decreased. Differences were also observed at t48 between the normal and high CHO diets in the abundance of Dorea, Ruminococcus and to a lesser degree Lachnospiraceae incertae sedis. At $72 \mathrm{~h}$ a slight change in the composition was observed in all three diets, but the overall pattern was similar to that observed at $48 \mathrm{~h}$. 
Rate of change

Using the weighted Unifrac distance analysis we observed that the largest change in composition occurred within the first $24 \mathrm{~h}$ of the fermentation of the diets (Fig. 9). The average Unifrac distance between $t 0$ and $t 24$ was significantly higher for the normal diet compared to the high protein diet $(\mathrm{p}<0.0001)$. For both microbiotas fermenting the high protein and the normal diet, they kept deviating from the composition at $\mathrm{t} 0$.

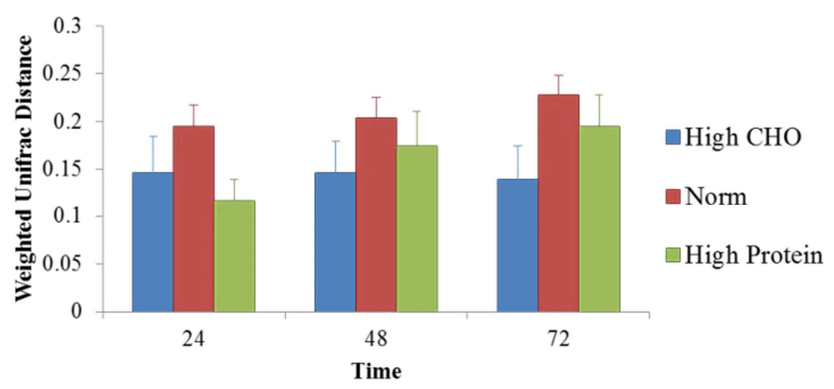

Fig. 9. Compositional difference between t0 at each timepoint for all diets. Unifrac distances were calculated between to and each timepoint.

Although the compositional changes based on weighted Unifrac seems to stagnate (especially for the microbiota fermenting the high $\mathrm{CHO}$ diet), the phylogenetic diversity showed an almost linear increase for all diets (Fig. 6). The microbiota fermenting the high protein diet showed the highest diversity, but also stabilized after t48. The diversity of the microbiota fermenting the high protein diet is higher when compared to the high $\mathrm{CHO}$ diet at all timepoints $(\mathrm{p}<0.0001)$

Metatranscriptomic analysis

KEGG functionality distance analysis showed clustering of samples based on functionality (Fig. 10). As observed for the compositional analysis, at t0 all the samples grouped together. From a total of 114573 genes, no single function was found to be differentially expressed between the high $\mathrm{CHO}$ and the high protein diet at this time point ( $\mathrm{t} 0$; Fig. S2a). After $24 \mathrm{~h}$ of administrating the different diets, differences were bigger in terms of functionality and samples were divided in two groups: i) one comprising the high $\mathrm{CHO}$ and the normal diet and ii) one which contains the high protein diet. Less than $9 \%$ of the functions analyzed were found to be differentially expressed in the high $\mathrm{CHO}$ and the high protein diet (t24; Fig. $\mathrm{S} 2 \mathrm{~b})$. 


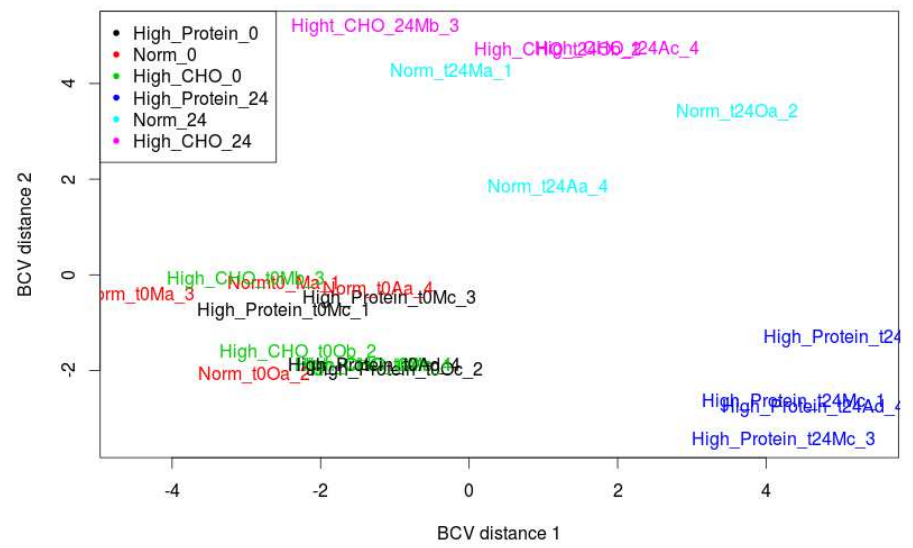

Fig. 10. KEGG functionality distance plot of timepoint t0 and t24.

\section{Discussion}

Multiple versions of in vitro gut models are currently available, all of them presenting different levels of complexity in their design. Some in vitro models consist of a simple batch culture, some others of sophisticated continuous multistage (semi) continuous cultures $[2,4,11]$. Both are characterized by the use of fecal microbiota as inoculum and their operation is strictly controlled. The more sophisticated models mostly performed under physiological temperature, $\mathrm{pH}$, food intake and anaerobic conditions [2,12].

Despite the efforts in closely simulating the human gut, in vitro gut models cannot be considered as an attempt to reproduce an exact replicate of the human intestine $[2,30]$. The complexity of host physiology is not fully reproduced in these models [30]. However, they constitute a potential tool to understand how fermentation in the human intestine occurs together with the possible mechanistic processes behind. Furthermore, the use of gut models clearly reduces laboratory animal use. Therefore, efforts should be focused on their adequate conditioning that ensures their repeatability, robustness and reproducibility for experimental studies [2].

The TNO dynamic in vitro model of the proximal colon (TIM-2) is one example of a widely used model for studying compositional and functional changes in the gut microbiota caused by different fermentable substrates. The operational conditions in TIM-2 are rigorously controlled and standardized. Furthermore, as described by Minekus et al., [31], the model was validated analyzing i) the composition, ii) the enzymatic activity and iii) production and concentration of SCFA from the microbiota with data from sudden death individuals. Thus, the system has been used for a wide range of analyses including the study of metabolism of phenolic 94 
compounds [32], the effect of prebiotics on the growth of bacteria $[13,15,17,33]$ and the use of different diets and intestinal microbiota [34,35].

Current new molecular techniques represent powerful instruments to demonstrate the reproducibility, robustness and repeatability of TIM-2. 16S rRNA sequencing and metatranscriptomics offer a high resolution to the understanding of the dynamics of specific microbial communities including the impact on their metabolic behavior [36-38]. Therefore, we compositionally (using 16S rRNA amplicon sequencing) and functionally (metabolite quantification and metastranscriptome sequencing) characterized the microbial community developing in TIM-2 in a $72 \mathrm{~h}$ fermentation on a normal diet, a diet high in $\mathrm{CHO}$ or a diet high in protein, and studied reproducibility of the system with 4 replicates per diet.

\section{Metabolites}

In this study, we observed that in terms of the metabolites produced, the dietary components (high $\mathrm{CHO}$ and high protein) were important determinants for the metabolic activity of the microbiota (Fig. 2, 3 and 4).

The high protein diet (with low carbohydrates) lead to a reproducible increased production of toxic metabolites (BCFA and ammonia) while the normal and high $\mathrm{CHO}$ diets yielded a reproducible higher production of health-promoting metabolites (SCFA). The differences in the total cumulative SCFA produced in the diets $(150.4 \pm 4.1 \mathrm{mmol}, 109.9 \pm 11.5 \mathrm{mmol}$ and $72.3 \pm 3.9 \mathrm{mmol}$ for normal, high $\mathrm{CHO}$ and high protein diets, respectively) presented little variation among replicates and the two very different versions of the diets: high $\mathrm{CHO}$ and high protein were statistically different. Our results corroborated previous reports indicating that the gut microbiota preferably ferment carbohydrates but switches to protein fermentation in case of carbohydrate depletion $[1,39,40]$. In addition, low production of SCFA can also indicate a less efficient fermentation and, consequently, a lower generation of energy for the host, as shown in the following chapters 6,7 and 8 .

\section{Microbiota profiling}

The observed decrease in the diversity index found between the t-16 and t0 samples, indicates that the microbiota was affected during the adaptation period (Fig. S1. In this regard, substrate limitation, dilution and product inhibition, $\mathrm{pH}$, redox potential and anaerobiosis may constitute the main causes that may limit the optimal propagation of the inoculum in an in vitro system [31,36,41].

Rajilic-Stojanovic et al. [42] also observed an initial drop in the microbial diversity (around 40\%) in TIM-2 samples during the adaptation period. Still, the authors did 
also find that the microbiota became more complex during the subsequent growth in the system, as also observed in the present study (Fig. 6, 7). In fact, the effect of the operational conditions on the development of the microbiota has been previously observed in other in vitro models of the human large intestine. For instance, SHIME has been shown to selectively stimulate the growth of Bacteroidetes and Clostridium cluster IX and reduce Clostridium clusters IV and XIVa [12]. Furthermore, in the present study we observed that the microbiota growing in the TIM-2 system presents a high abundance of Streptococcus and Lactococcus which is not representative for the colon.

Despite the transition of the microbiota from gut to in vitro, the development of the communities in the TIM-2 seemed to recover and showed to consistently increase in diversity through time suggesting that possibly the operational conditions of the system: strict $\mathrm{pH}$ control, continuous nutrient administration and removal of microbial metabolites (dialysis system) promoted the gradual growth of a complex community (Fig. 6, 7). Furthermore, after the adaptation period, the composition of samples from all 12 experiments at t0 showed highly similar communities when compared to each other (Fig. 5) which evidenced that the composition of the community seemed to be similarly affected in all experiments during the adaptation period, again indicating reproducibility.

Streptococcus, and Lactococcus were found to be highly abundant in the microbial community at $\mathrm{t} 0$. These are well-known genera of lactic acid bacteria previously found to grow in the TIM-2 model [43]. The use of a complex growth medium (such as SIEM) with a high amount of carbohydrates (especially starch), proteins, vitamins and minerals may have particularly increased the growth of this group of bacteria during the adaptation period. However, the decrease observed in the subsequent sampling times ( $\mathrm{t} 24,48$ and 72) may indicate that their growth dynamics could have been affected by consistently providing the diets either with fermentable $\mathrm{CHO}$ or high in protein content.

Development of the community structure during fermentation of the diets in TIM-2 As observed before, our data suggests the effect of dietary components as a major determinant in the composition of gut microbiota. After initiating the feeding with the different diets, it was possible to observe that specific subpopulations of the microbiota seemed to be reproducibly affected depending on the composition of the diet (Fig. 5-7).

In agreement with the production of the different metabolites, the composition of the microbiota fermenting the normal and the high $\mathrm{CHO}$ presented more similarities when compared to each other than when compared to the microbiota fermenting the high protein diet. 
The high $\mathrm{CHO}$ and the normal diet were observed to increase the abundance of Subdoligranulum. Our findings are in agreement with another study in which a low protein diet was found to increase the abundance of Subdoligranulum [44]. Furthermore, this group of bacteria has been reported to be highly abundant in vegetarians [45].

Blautia was also found to be highly abundant in the microbiota fermenting the normal and the high $\mathrm{CHO}$ diet. This finding is expected since the total human microbiome is estimated to comprise between 2.5 to $16 \%$ of Blautia spp. members [46]. Moreover, this genus has been positively correlated with the fermentation of resistant starch (which was more available in these diets) suggesting this genus to play an important role in starch degradation [47].

A microbial community high in Prevotella was also found in the experiments with the normal and high $\mathrm{CHO}$ diet. The Prevotella genus is known to degrade carbohydrates, both insoluble plant fiber and soluble carbohydrates $[48,49]$. Furthermore, an increase of Bifidobacterium populations was also observed in the microbiota fermenting these diets. Usually the increase in Bifidobacterium is associated with supplementation of galactooligosaccharides (GOS) and/or fructooligosaccharides (FOS) [50,51]. However, data from several studies suggest that Bifidobacterium species also have the ability to use starch to grow $[52,53]$ and to primarily consume the intermediates produced by Ruminococcus related species (which were also detected in our study) during the primary degradation of starch [53].

The increase in Bacteroides observed in the diet with a low carbohydrate (but relatively higher protein) content is consistent with the nutrient preferences reported for this genus. A long-term diet rich in protein has been suggested to be dominated by Bacteroides [54,55]. Furthermore, levels of Bacteroides have been observed to increase after a shift in $\mathrm{pH}$ (5.5 to 6.5) in cultures supplied with peptides and a mixed carbohydrate source [56]. Interestingly, Lachnospiraceae incertae sedis was also observed to increase in the microbiota fermenting the high protein diet. This has been previously described as an emerging taxon of interest due to its potential role in irritable bowel syndrome (IBS) which suggests future research about the importance of these bacteria and their association with health status [57].

Dorea spp. have been found to be carbohydrate utilizing bacteria [58]. In this study, it was found to increase through time when fermenting the high protein diet. However, these bacteria also increased in the normal diet which also indicates more research is needed in order to elucidate their impact on gut health. 
Metatranscriptomics

It is a challenge to define when a community stabilizes since it is questionable how much variation can be accepted to reach such stability [59]. However, our weighted Unifrac distance analysis showed that there is a fast change in the microbiota composition between $\mathrm{t} 0$ and $\mathrm{t} 24$, especially in the microbiota fed with the high protein diet (Fig. 9). After this time it seemed that the microbiota tended to stabilize. This is in agreement with previous observations by us and others about the resilience of the gut microbiota $[40,60]$.

Based on these findings we zoomed in to the community gene expression level at t0 and t24. Again, we observed a consistent robust and reproducible response in all diets (Fig. 10). The fact that we did not find any differentially expressed functions at t0 matched our expectations of reproducibility, allowing us to verify that after the adaptation period the microbiota grew consistently in the system and the starting point for every of the 12 experiments is very similar even at the gene expression level.

We also expected a low abundance of differentially expressed genes after $24 \mathrm{~h}$. As previously observed, there are household genes in the microbiota that are expressed similarly under multiple different conditions [26] revealing high degree of similarity on metatranscriptomic profiles. This must mean that the genes that are differentially expressed on the different diets are important under the new dietary regimes. Detailed characterization of those genes fell outside the scope of this reproducibility study and will be reported elsewhere.

\section{Conclusion}

The effects of different diets on the microbiota composition and activity were repeatable, robust and reproducible in the different replicates through all experimentation weeks. This is demonstrated statistically and is reflected even at the gene expression level. Our findings not only showed the gut microbiota as a rapidly metabolically adaptable organ as previously reported [40,60] but also highlighted the sensitivity and the potential of the TIM-2 system as a tool for investigating the role of the microbiota on human health or disease through investigating reproducible changes in community structure and activity.

\section{Acknowledgments}

This study was partly funded by the Top Institute Food \& Nutrition (TIFN, Wageningen, The Netherlands) under project GH004. 


\section{References}

1. Egert M, de Graaf AA, Smidt H, de Vos WM, Venema K (2006) Beyond diversity: functional microbiomics of the human colon. Trends Microbiol 14: 86-91.

2. McDonald JA, Schroeter K, Fuentes S, Heikamp-Dejong I, Khursigara CM, et al. (2013) Evaluation of microbial community reproducibility, stability and composition in a human distal gut chemostat model. J Microbiol Methods 95: 167-174.

3. Venema K, van den Abbeele P (2013) Experimental models of the gut microbiome. Best Pract Res Clin Gastroenterol 27: 115-126.

4. Payne AN, Zihler A, Chassard C, Lacroix C (2012) Advances and perspectives in in vitro human gut fermentation modeling. Trends Biotechnol 30: 17-25.

5. Van de Wiele T, Vanhaecke L, Boeckaert C, Peru K, Headley J, et al. (2005) Human colon microbiota transform polycyclic aromatic hydrocarbons to estrogenic metabolites. Environ Health Perspect 113: 6-10.

6. Van de Wiele TR, Verstraete W, Siciliano SD (2004) Polycyclic aromatic hydrocarbon release from a soil matrix in the in vitro gastrointestinal tract. $J$ Environ Qual 33: 1343-1353.

7. Sivieri K, Morales ML, Saad SM, Adorno MA, Sakamoto IK, et al. (2014) Prebiotic effect of fructooligosaccharide in the simulator of the human intestinal microbial ecosystem (SHIME(R) model). J Med Food 17: 894-901.

8. Aguirre M, Jonkers DM, Troost FJ, Roeselers G, Venema K (2014) In vitro characterization of the impact of different substrates on metabolite production, energy extraction and composition of gut microbiota from lean and obese subjects. PLoS One 9: e113864.

9. Vesterlund S, Paltta J, Karp M, Ouwehand AC (2005) Measurement of bacterial adhesion in vitro evaluation of different methods. J Microbiol Methods 60: 225-233.

10. Nuck B, Schlagheck T, Federle T (1994) Inability of the human fecal microflora to metabolize the nonabsorbable fat substitute, olestra. J Ind Microbiol 13: 328334.

11. Macfarlane GT, Macfarlane S (2007) Models for intestinal fermentation: association between food components, delivery systems, bioavailability and functional interactions in the gut. Curr Opin Biotechnol 18: 156-162.

12. Van den Abbeele P, Grootaert C, Marzorati M, Possemiers S, Verstraete W, et al. (2010) Microbial community development in a dynamic gut model is reproducible, colon region specific, and selective for Bacteroidetes and Clostridium cluster IX. Appl Environ Microbiol 76: 5237-5246.

13. Maathuis AJH, van den Heuvel EG, Schoterman MHC, Venema K (2012) GalactoOligosaccharides Have Prebiotic Activity in a Dynamic in vitro Colon Model Using a C-Labeling Technique. J Nutr 142: 1205-1212. 
14. Aguirre M, Ramiro-Garcia J, Koenen ME, Venema K (2014) To pool or not to pool? Impact of the use of individual and pooled fecal samples for in vitro fermentation studies. J Microbiol Methods 107C: 1-7.

15. Maathuis A, Hoffman A, Evans A, Sanders L, Venema K (2009) The effect of the undigested fraction of maize products on the activity and composition of the microbiota determined in a dynamic in vitro model of the human proximal large intestine. J Am Coll Nutr 28: 657-666.

16. de Graaf AA, Maathuis A, de Waard P, Deutz NE, Dijkema C, et al. (2010) Profiling human gut bacterial metabolism and its kinetics using [U13C]glucose and NMR. NMR Biomed 23: 2-12.

17. van Nuenen MHMC, Diederick Meyer P, Venema K (2003) The Effect of Various Inulins and Clostridium difficile on the Metabolic Activity of the Human Colonic Microbiota in vitro. Microb Ecol Health Dis 15: 137-144.

18. Tabernero M, Venema K, Maathuis AJ, Saura-Calixto FD (2011) Metabolite production during in vitro colonic fermentation of dietary fiber: analysis and comparison of two European diets. J Agric Food Chem 59: 8968-8975.

19. Caporaso JG, Lauber CL, Walters WA, Berg-Lyons D, Lozupone CA, et al. (2011) Global patterns of $16 \mathrm{~S}$ rRNA diversity at a depth of millions of sequences per sample. Proc Natl Acad Sci U S A 108 Suppl 1: 4516-4522.

20. Claesson MJ, Wang Q, O'Sullivan O, Greene-Diniz R, Cole JR, et al. (2010) Comparison of two next-generation sequencing technologies for resolving highly complex microbiota composition using tandem variable 16S rRNA gene regions. Nucleic Acids Res 38: e200.

21. Walters WA, Caporaso JG, Lauber CL, Berg-Lyons D, Fierer N, et al. (2011) PrimerProspector: de novo design and taxonomic analysis of barcoded polymerase chain reaction primers. Bioinformatics 27: 1159-1161.

22. Caporaso JG, Kuczynski J, Stombaugh J, Bittinger K, Bushman FD, et al. (2010) QIIME allows analysis of high-throughput community sequencing data. Nat Methods 7: 335-336.

23. Zoetendal EG, Booijink CC, Klaassens ES, Heilig HG, Kleerebezem M, et al. (2006) Isolation of RNA from bacterial samples of the human gastrointestinal tract. Nat Protoc 1: 954-959.

24. Murphy NR, Hellwig RJ (1996) Improved nucleic acid organic extraction through use of a unique gel barrier material. Biotechniques 21: 934-936, 938-939.

25. Kopylova E, Noe L, Touzet H (2012) SortMeRNA: fast and accurate filtering of ribosomal RNAs in metatranscriptomic data. Bioinformatics 28: 3211-3217.

26. Leimena MM, Ramiro-Garcia J, Davids M, van den Bogert B, Smidt H, et al. (2013) A comprehensive metatranscriptome analysis pipeline and its validation using human small intestine microbiota datasets. BMC Genomics 14: 530.

27. Davids M, Hugenholtz F, Martins dos Santos V, Smidt H, Kleerebezem M, et al. (2016) Functional Profiling of Unfamiliar Microbial Communities Using a 
Validated de novo Assembly Metatranscriptome Pipeline. PLoS ONE 11: $\mathrm{e} 0146423$.

28. Robinson MD, McCarthy DJ, Smyth GK (2010) edgeR: a Bioconductor package for differential expression analysis of digital gene expression data. Bioinformatics 26: 139-140.

29. Faith DP (2006) The role of the phylogenetic diversity measure, PD, in bioinformatics: getting the definition right. Evol Bioinform Online 2: 277-283.

30. Payne AN, Chassard C, Banz Y, Lacroix C (2012) The composition and metabolic activity of child gut microbiota demonstrate differential adaptation to varied nutrient loads in an in vitro model of colonic fermentation. FEMS Microbiol Ecol 80: 608-623.

31. Minekus M, Smeets-Peeters M, Bernalier A, Marol-Bonnin S, Havenaar R, et al. (1999) A computer-controlled system to simulate conditions of the large intestine with peristaltic mixing, water absorption and absorption of fermentation products. Appl Microbiol Biotechnol 53: 108-114.

32. Gao K, Xu A, Krul C, Venema K, Liu Y, et al. (2006) Of the major phenolic acids formed during human microbial fermentation of tea, citrus, and soy flavonoid supplements, only 3,4-dihydroxyphenylacetic acid has antiproliferative activity. J Nutr 136: 52-57.

33. Martinez RC, Cardarelli HR, Borst W, Albrecht S, Schols H, et al. (2013) Effect of galactooligosaccharides and Bifidobacterium animalis Bb-12 on growth of Lactobacillus amylovorus DSM 16698, microbial community structure, and metabolite production in an in vitro colonic model set up with human or pig microbiota. FEMS Microbiol Ecol 84: 110-123.

34. Rose DJ, Venema K, Keshavarzian A, Hamaker BR (2010) Starch-entrapped microspheres show a beneficial fermentation profile and decrease in potentially harmful bacteria during in vitro fermentation in faecal microbiota obtained from patients with inflammatory bowel disease. Br J Nutr 103: 15141524 .

35. Leijdekkers AGM, Aguirre M, Venema K, Bosch G, Gruppen H, et al. (2014) In Vitro Fermentability of Sugar Beet Pulp Derived Oligosaccharides Using Human and Pig Fecal Inocula. J Agric Food Chem 62: 1079-1087.

36. Feria-Gervasio D, Denis S, Alric M, Brugere JF (2011) In vitro maintenance of a human proximal colon microbiota using the continuous fermentation system PECSIM. Appl Microbiol Biotechnol 91: 1425-1433.

37. Qin J, Li R, Raes J, Arumugam M, Burgdorf KS, et al. (2010) A human gut microbial gene catalogue established by metagenomic sequencing. Nature $\mathbf{4 6 4}$ : 59-65.

38. Gill SR, Pop M, Deboy RT, Eckburg PB, Turnbaugh PJ, et al. (2006) Metagenomic analysis of the human distal gut microbiome. Science 312: 1355-1359. 
39. Ouwehand AC, Derrien M, de Vos W, Tiihonen K, Rautonen N (2005) Prebiotics and other microbial substrates for gut functionality. Curr Opin Biotechnol 16: 212-217.

40. Aguirre M, Eck A, Koenen ME, Savelkoul PH, Budding AE, et al. (2015) Diet drives quick changes in the metabolic activity and composition of human gut microbiota in a validated in vitro gut model. Res Microbiol 167: 114-125.

41. Aguirre M, Eck A, Koenen ME, Savelkoul PH, Budding AE, et al. (2015) Evaluation of an optimal preparation of human standardized fecal inocula for in vitro fermentation studies. J Microbiol Methods 117: 78-84.

42. Rajilic-Stojanovic M, Maathuis A, Heilig HG, Venema K, de Vos WM, et al. (2010) Evaluating the microbial diversity of an in vitro model of the human large intestine by phylogenetic microarray analysis. Microbiology 156: 32703281.

43. Egert M, de Graaf AA, Maathuis A, de Waard P, Plugge CM, et al. (2007) Identification of glucose-fermenting bacteria present in an in vitro model of the human intestine by RNA-stable isotope probing. FEMS Microbiol Ecol 60: 126-135.

44. Zhou L, Fang L, Sun Y, Su Y, Zhu W (2016) Effects of the dietary protein level on the microbial composition and metabolomic profile in the hindgut of the pig. Anaerobe 38: 61-69.

45. Matijasic BB, Obermajer T, Lipoglavsek L, Grabnar I, Avgustin G, et al. (2014) Association of dietary type with fecal microbiota in vegetarians and omnivores in Slovenia. Eur J Nutr 53: 1051-1064.

46. Zoetendal EG, Raes J, van den Bogert B, Arumugam M, Booijink CC, et al. (2012) The human small intestinal microbiota is driven by rapid uptake and conversion of simple carbohydrates. ISME J 6: 1415-1426.

47. Yang J, Martinez I, Walter J, Keshavarzian A, Rose DJ (2013) In vitro characterization of the impact of selected dietary fibers on fecal microbiota composition and short chain fatty acid production. Anaerobe 23: 74-81.

48. Andrés M, Ana D, Juan José A, Amparo L (2013) Effect of dietary carbohydrate restriction on an obesity-related Prevotella-dominated human faecal microbiota. Metagenomics 2013.

49. Flint HJ, Bayer EA, Rincon MT, Lamed R, White BA (2008) Polysaccharide utilization by gut bacteria: potential for new insights from genomic analysis. Nat Rev Microbiol 6: 121-131.

50. Akiyama T, Kimura K, Hatano H (2015) Diverse galactooligosaccharides consumption by bifidobacteria: implications of beta-galactosidase-LacS operon. Biosci Biotechnol Biochem 79: 664-672.

51. Bouhnik Y, Flourie B, Riottot M, Bisetti N, Gailing MF, et al. (1996) Effects of fructo-oligosaccharides ingestion on fecal bifidobacteria and selected 
Reproducibility of the TIM-2 system

metabolic indexes of colon carcinogenesis in healthy humans. Nutr Cancer 26: 21-29.

52. Walker AW, Ince J, Duncan SH, Webster LM, Holtrop G, et al. (2011) Dominant and diet-responsive groups of bacteria within the human colonic microbiota. The ISME journal 5: 220-230.

53. Kovatcheva-Datchary P, Egert M, Maathuis A, Rajilic-Stojanovic M, de Graaf AA, et al. (2009) Linking phylogenetic identities of bacteria to starch fermentation in an in vitro model of the large intestine by RNA-based stable isotope probing. Environ Microbiol 11: 914-926.

54. Wu GD, Chen J, Hoffmann C, Bittinger K, Chen Y-Y, et al. (2011) Linking LongTerm Dietary Patterns with Gut Microbial Enterotypes. Science 334: 105-108.

55. Zimmer J, Lange B, Frick JS, Sauer H, Zimmermann K, et al. (2012) A vegan or vegetarian diet substantially alters the human colonic faecal microbiota. Eur $J$ Clin Nutr 66: 53-60.

56. Walker AW, Duncan SH, Leitch ECM, Child MW, Flint HJ (2005) pH and peptide supply can radically alter bacterial populations and short-chain fatty acid ratios within microbial communities from the human colon. Appl Environ Microbiol 71: 3692-3700.

57. Salonen A, de Vos W, Palva A (2010) Gastrointestinal microbiota in irritable bowel syndrome: present state and perspectives. Microbiology 156: 32053215.

58. Taras D, Simmering R, Collins MD, Lawson PA, Blaut M (2002) Reclassification of Eubacterium formicigenerans Holdeman and Moore 1974 as Dorea formicigenerans gen. nov., comb. nov., and description of Dorea longicatena sp. nov., isolated from human faeces. Int J Syst Evol Microbiol 52: 423-428.

59. Auchtung JM, Robinson CD, Britton RA (2015) Cultivation of stable, reproducible microbial communities from different fecal donors using minibioreactor arrays (MBRAs). Microbiome 3: 1-15.

60. Salonen A, Lahti L, Salojarvi J, Holtrop G, Korpela K, et al. (2014) Impact of diet and individual variation on intestinal microbiota composition and fermentation products in obese men. ISME J 8: 2218-2230. 


\section{Supplemental material}

Table S1. Fermentation metabolites produced during $72 \mathrm{~h}$ fermentation experiments.

\begin{tabular}{clcccc}
\multirow{2}{*}{ Diet } & & \multicolumn{4}{c}{ mmol } \\
\cline { 3 - 6 } Normal & acetate & 0 & $\mathbf{t}$ t24 & $\mathbf{t 4 8}$ & $\mathbf{t 7 2}$ \\
& propionate & 0 & $7.49 \pm 3.10$ & $55.9 \pm 3.94$ & $83.1 \pm 7.54$ \\
& $n$-butyrate & 0 & $7.51 \pm 0.70$ & $18.7 \pm 6.16$ & $34.7 \pm 7.51$ \\
& Total SCFA & 0 & $41.8 \pm 3.12$ & $95.0 \pm 2.10$ & $150.4 \pm 4.09$ \\
& ammonia & 0 & $12.6 \pm 1.56$ & $32.1 \pm 2.08$ & $54.5 \pm 2.62$ \\
& iso-butyrate & 0 & $0.15 \pm 0.13$ & $0.45 \pm 0.27$ & $1.01 \pm 0.31$ \\
& iso-valerate & 0 & $0.31 \pm 0.20$ & $0.87 \pm 0.27$ & $1.86 \pm 0.28$ \\
& Total BCFA & 0 & $0.45 \pm 0.32$ & $1.32 \pm 0.54$ & $2.87 \pm 0.57$ \\
& Alkali & $13.88 \pm 1.91$ & $36.3 \pm 4.85$ & $53.9 \pm 7.13$ & $69.4 \pm 7.86$ \\
High CHO & acetate & 0 & $27.2 \pm 4.89$ & $47.6 \pm 7.71$ & $70.4 \pm 8.54^{*}$ \\
& propionate & 0 & $3.73 \pm 1.49$ & $8.77 \pm 2.56^{*}$ & $13.7 \pm 3.59^{*}$ \\
& $n$-butyrate & 0 & $7.38 \pm 1.76$ & $15.8 \pm 1.97$ & $25.8 \pm 2.02^{*}$ \\
& Total SCFA & 0 & $38.3 \pm 4.88$ & $72.3 \pm 8.37^{*}$ & $109.9 \pm 11.5^{*}$ \\
& ammonia & 0 & $3.17 \pm 0.83^{*}$ & $5.48 \pm 1.38^{*}$ & $8.12 \pm 1.95^{*}$ \\
& iso-butyrate & 0 & $0.00 \pm 0$ & $0.00 \pm 0^{*}$ & $0.07 \pm 0.14^{*}$ \\
& iso-valerate & 0 & $0.00 \pm 0$ & $0.02 \pm 0.02^{*}$ & $0.16 \pm 0.17^{*}$ \\
& Total BCFA & 0 & $0.00 \pm 0$ & $0.02 \pm 0.02^{*}$ & $0.23 \pm 0.29^{*}$ \\
High & Alkali & $13.33 \pm 2.38$ & $39.1 \pm 3.54$ & $60.2 \pm 4.66$ & $84.0 \pm 6.76^{*}$ \\
protein & & & $12.4 \pm$ & & \\
& acetate & 0 & $1.35^{* \dagger}$ & $24.7 \pm 1.57^{* \dagger}$ & $37.8 \pm 2.47^{* \dagger}$ \\
& propionate & 0 & $6.24 \pm 1.08$ & $13.4 \pm 2.52$ & $20.7 \pm 3.12^{*}$ \\
& $n$-butyrate & 0 & $4.24 \pm 0.76$ & $8.50 \pm 1.82^{* \dagger}$ & $13.8 \pm 3.00^{* \dagger}$ \\
& & & $22.9 \pm$ & & \\
& Total SCFA & 0 & $2.29^{* \dagger}$ & $46.8 \pm 3.44^{* \dagger}$ & $72.3 \pm 3.85^{* \dagger}$ \\
& & & $19.9 \pm$ & & \\
& ammonia & 0 & $2.74^{* \dagger}$ & $47.5 \pm 4.05^{* \dagger}$ & $79.7 \pm 3.78^{* \dagger}$ \\
& iso-butyrate & 0 & $0.24 \pm 0.17$ & $0.86 \pm 0.12^{* \dagger}$ & $1.86 \pm 0.16^{* \dagger}$ \\
& iso-valerate & 0 & $0.65 \pm 0.17^{\dagger}$ & $1.80 \pm 0.18^{* \dagger}$ & $3.47 \pm 0.25^{* \dagger}$ \\
& Total BCFA & 0 & $0.89 \pm 0.33^{\dagger}$ & $2.66 \pm 0.29^{* \dagger}$ & $5.33 \pm 0.36^{* \dagger}$ \\
& & & $16.1 \pm$ & & \\
& Alkali & $14.28 \pm 1.80$ & $2.40^{* \dagger}$ & $16.1 \pm 2.40^{* \dagger}$ & $16.1 \pm 2.40^{* \dagger}$
\end{tabular}

${ }^{1}$ Key: $* p<0.01$ specific time point is significantly different compared to the normal diet; $\dagger p<0.01$ specific time point is significantly different compared to the high $\mathrm{CHO}$ diet. The 
different diets correspond to: normal: 1:1 (control or basal SIEM medium); high CHO: 10:1 and high protein 1:10 (CHO: protein) diets.
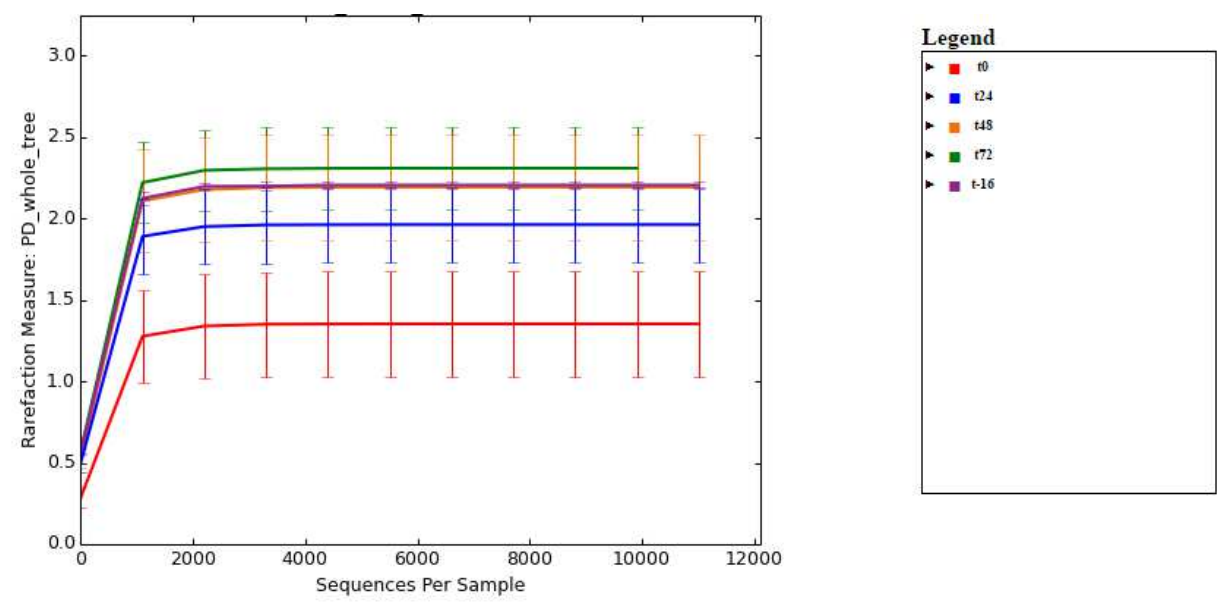

Fig. S1. PD whole tree ( $\alpha$-diversity) from TIM-2 samples through time.

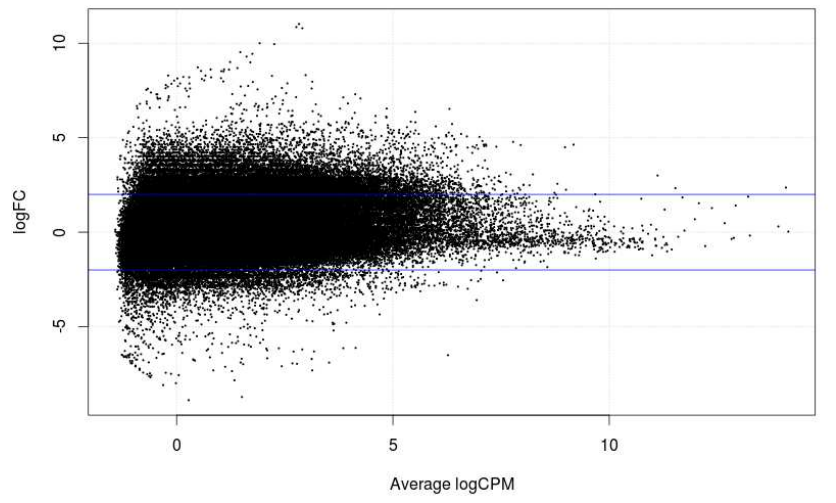

Fig. S2a. Differentially expressed functions between the high $\mathrm{CHO}$ and the high protein diet at $\mathrm{t} 0$. 


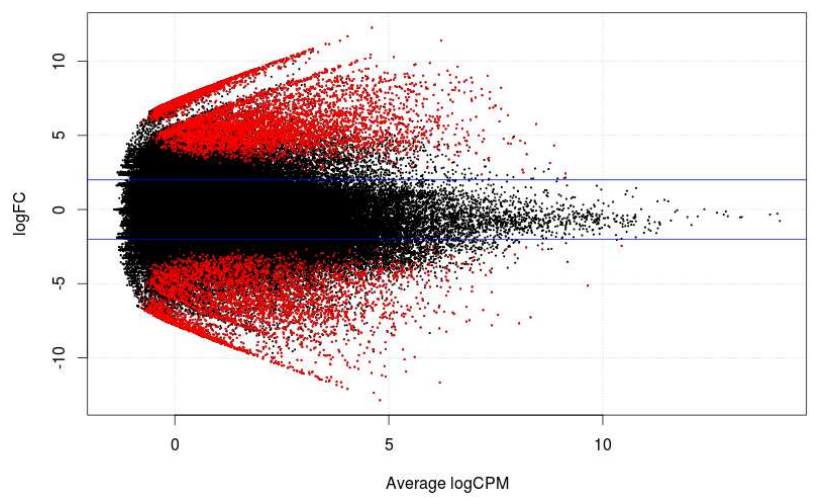

Fig. S2b. Differentially expressed functions between high $\mathrm{CHO}$ and the high protein diet at $\mathrm{t} 24$. 


\title{
Chapter 5
}

\author{
Diet drives quick changes in the metabolic \\ activity and composition of human gut \\ microbiota in a validated in vitro gut model
}

Aguirre M, Eck A, Savelkoul PHM, Budding AE, Venema K (2016) Diet drives quick changes in the metabolic activity and composition of human gut microbiota in a validated in vitro gut model Res Microbiol 167 (2016) pp. 114-125 


\section{Abstract}

The aim of this study was to screen how quick the human gut microbiota responds to diet in an in vitro model of the proximal colon (TIM-2 system). Two experimental diets were provided to the gut bacteria: a high carbohydrate and a high protein diet. The metabolic response and the composition of the microbiota were compared to a control diet simulating an average western meal. Short-chain and branched-chain fatty acids (SCFA and BCFA, respectively) production, in addition to changes in the community composition (profiling), were measured. The activity of the microbiota reflected differences between the diets exhibiting a tradeoff between saccharolytic and proteolytic fermentation when compared to the control. Diversity analysis revealed a phylum-specific response depending on the diet tested. Most changes in the microbiome composition occurred during the first $24 \mathrm{~h}$ of the experiment. The outcome of this study allowed to elucidate that the human gut bacteria quickly respond to changes in diet. In addition, it confirms that variations in the concentration of carbohydrates and proteins modify the activity and composition of the microbiota and these changes can potentially have an impact on the health of the host. 
Diet fermentation in TIM-2

\section{Introduction}

Increasing evidence shows that varied nutrient loads and nutritional ingredients influence the composition and the production of metabolites by the gut microbiota, which has been shown to be involved in obesity [1], increased risk of colon cancer [2] and atherosclerosis [3], although the exact mechanism(s) so far are unclear.

Studies with mice have indicated that a high-fat, high-sugar or low-fiber diet may induce an aberrant microbial composition which may be related to high inflammation and increased intestinal permeability [4]. Long-term diets have also been observed to shape the composition of the human gut microbiota. A clinical study performed in humans showed that long-term diets influenced the so-called enterotypes [5]. The enterotype concept consists of clustering the gut bacterial community in three different consortia: Bacteroides-, Prevotella- or Ruminococcus-enriched, respectively. Bacteroides enterotype has been found in subjects with a diet rich in animal fat, protein and saturated fats, whereas a diet low in these dietary components but rich in carbohydrates and simple sugars has been associated with the Prevotella enterotype [5]. However, the classification of microbial profiles into distinct categories such as the so-called enterotypes has been highly criticized. The discussion is mainly based on the fact that these bacterial clusters are oversimplified, neglecting the complexity and uniqueness of the human microbiota.

Currently, there is no consensus on how fast and reproducibe the human gut microbiota can respond to short-term changes in the diet and there is scarce available information addressing this question. One of the few studies was performed by David et al. [6] who elegantly demonstrated that the microbiota can rapidly respond during the exposure of five consecutive days to an altered diet and how this triggered changes in microbial gene expression.

Together with alterations in the composition of the microbiota it has been observed that its metabolic activity changes as well. For instance, Duncan et al. [7] found a decrease in the production of short-chain fatty acids (SCFA) mainly acetate, butyrate and valerate in feces from obese individuals during consumption of a high protein/medium carbohydrate and a high protein/low carbohydrate diet. Furthermore, a study evaluating the impact of different substrates on the microbiota from lean and obese subjects demonstrated differences in metabolite production accompanied by changes in energy extraction [8].

Due to these microbiota-host and -diet interactions, strategies using dietary interventions can be used to tackle, prevent or even treat worldwide epidemics such as obesity [9-11]. To this end, more information is required about how different 
and how fast dietary compounds affect the composition and activity of the human gut microbiota.

Studies performed in human individuals are the ideal way to assess these types of effects. However, the limitation of performing these experiments are mainly attributed to ethical concerns, difficulties in sampling, and the high costs associated with these studies. An alternative to this is the performance of in vitro studies, which in addition offer the possibility to fill the gap in knowledge of the mechanisms behind end point observations in humans such as weight loss, reduced body mass, improvement of insulin sensitivity, etc.

The TIM-2 system, developed by The Netherlands Organization for Applied Scientific Research (TNO) [12], represents a powerful, reproducible and validated tool to monitor the fermentation of dietary compounds using human feces as an inoculum accompanied by the assessment of metabolites such as SCFA and other bacterial products.

This study aimed to provide insight about how fast changes in bacterial activity and composition can be induced in a $72 \mathrm{~h}$ fermentation using the validated TIM-2 system when major modifications in carbohydrate and protein content were supplied in the diet provided to the microbiota. To this end, profiling of the microbiota by means of IS-pro [13] was employed to determine the changes in the community upon the assimilation of the different diets.

\section{Materials and methods}

\section{Microbiota source}

A standardized homogenate was prepared from fecal donations from a group of 10 healthy volunteers (age range 31-57 y; 8 male, 2 female; BMI $23.3 \pm 2.7 \mathrm{~kg} / \mathrm{m}^{2}$ ). Individuals provided a signed informed consent prior to participation and were non-smokers and had not used antibiotics, prebiotics, probiotics or laxatives 1 month before the donation. Feces samples were pooled and inoculated $(44 \mathrm{~h})$ in a batch fermentor which simulated the conditions in the cecum as described by Venema et al. [14]. The extent to which this standardization procedure affects the composition of the microbiota has been previously studied. Despite that diversity declined in the standardized inoculum when compared to the feces from which it was derived, the standardized microbiota was more similar to fecal samples than to microbiota from the small intestine [15].

Aliquots from the microbiota obtained after such standardization were snap-frozen in liquid nitrogen and stored in a freezer at $-80{ }^{\circ} \mathrm{C}$. Before being introduced into the system, the inoculum was thawed by $1 \mathrm{~h}$ immersion in a $37^{\circ} \mathrm{C}$ water bath. 
Diet fermentation in TIM-2

Substrate used for fermentation: source and preparation

During the adaptation period ( $16 \mathrm{~h}$ ) all TIM-2 units were fed with simulated ileal efflux medium (SIEM) as described by Maathuis et al.[16]. After this adaptation period the units were fed either with two experimental diets or SIEM (control) which simulates the undigested part and nutrients characteristic of a western diet [17]. The two experimental diets that were studied were modifications of this control medium and differed in the carbohydrate and protein content as described next:

Normal diet; 1:1 (CHO: protein)

A feeding containing basal medium (normal preparation of SIEM) was used as a control. The composition of this medium has been described before [16]. Briefly, it contains TBCO (mixture (g/L) of Tween 80 (34.0), casein (47.0), bacto-peptone (47.0), and ox bile (0.8)); CHO (pectin (9.4), xylan (9.4), arabinogalactan (9.4), amylopectin (9.4) and starch (78.4)); and a vitamin mixture (containing (per liter) 1 $\mathrm{mg}$ menadione, $2 \mathrm{mg}$ D-biotin, $0.5 \mathrm{mg}$ vitamin B12, $10 \mathrm{mg}$ pantothenate, $5 \mathrm{mg}$ nicotinamide, $5 \mathrm{mg}$ p-aminobenzoic acid and $4 \mathrm{mg}$ thiamine).

High carbohydrate diet; 10:1 (CHO: protein)

The preparation of SIEM was modified by diluting 10 times the concentration of TBCO while keeping the proportions of the rest of the ingredients in the basal medium the same.

High protein diet; 1:10 (CHO: protein)

The content of carbohydrates in the basal medium was diluted 10 times. The amounts of the rest of the ingredients were the same as in the basal medium.

\section{TIM-2 experimental protocol}

The TIM-2 system was flushed with $\mathrm{N}_{2}$ prior to the introduction of the inoculum and it was maintained under this condition at $37{ }^{\circ} \mathrm{C}$ for $96 \mathrm{~h}$ with the $\mathrm{pH}$ kept at or above 5.8 by automatic titration with $2 \mathrm{M} \mathrm{NaOH}$. In order to remove water and fermentation products from the lumen, a dialysate system (described in detail by van Nuenen et al. [18]) consisting of a semi-permeable hollow membrane ran through the lumen. For all the experiments, the speed of the dialysis fluid was set at $1.5 \mathrm{ml} / \mathrm{min}$. After 24 and $48 \mathrm{~h}$ of fermentation $25 \mathrm{ml}$ of lumen sample was removed from the system to mimic the transit of material from the proximal to the distal colon [16]. The basal medium preparation (SIEM) was gradually introduced into the system in a total volume of $40 \mathrm{ml}$ during the adaptation period (see below), and $180 \mathrm{ml}$ of the different experimental diets or control over the $72 \mathrm{~h}$ of the test 
period at a rate of $2.5 \mathrm{ml} / \mathrm{h}$. Luminal content was maintained at a level of approximately $120 \mathrm{ml}$ in each unit by a level sensor (liquiphant FTL20-0025, Endress+Hauser). Details of the system have been described before [16].

\section{Design of the experiments}

A $30 \mathrm{ml}$ portion of fecal homogenate was used to inoculate the units for each experiment. Each unit was then filled to $120 \mathrm{ml}$ with $90 \mathrm{ml}$ of dialysate [16]. Right after the inoculation, the microbiota was left to adapt $(16 \mathrm{~h})$ to the new environment. After this adaptation period, the culture was deprived from any medium for $3 \mathrm{~h}$ (starvation) in order to maximize test substrates use by the microbiota which permits a better detection sensitivity of the effect of the test substrates in the media [19]. Since TIM-2 is a computer controlled model and it has been shown to be highly reproducible during the last two decades [16,20,21], experiments were performed in series of two per diet formulation $(n=2)$. Due to the small sample size no statistical analysis was executed. Luminal and dialysate sampling were performed at $0,24,48$ and $72 \mathrm{~h}$. In both cases samples were stored $\left(-80{ }^{\circ} \mathrm{C}\right)$ until analysis.

\section{Analytical methods}

Metabolite production

SCFA (acetate, propionate, and $n$-butyrate) and BCFA (iso-butyrate and isovalerate) samples were prepared and analyzed by gas chromatography as previously described by van Nuenen et al. [18]. Calibration lines were obtained by injecting known quantities of standard solutions, and amounts in the samples were calculated based on the calibration lines.

Data presentation

The standard deviation based on the entire population was calculated, data are shown as average \pm SD. To avoid unnecessary repetition, this is not indicated further in the text or graphs in the results section.

Characterization of bacterial populations by IS-pro

DNA from the luminal samples was isolated using the AGOWA mag Mini kit (DNA Isolation Kit, AGOWA, Berlin, Germany), according to the manufacturer's instructions.

Amplification of ribosomal interspacer (IS)-regions was performed with the IS-pro kit (IS-diagnostics, Amsterdam, The Netherlands). IS-pro differentiates bacterial taxa on phylum level by phylum-specific fluorescent labeled primers. Then, differentiation up to species level can be done by analysis of the length of 
amplicons. Each bacterial species has its unique signature of IS-fragment lengths, which is highly differentiating (>99\% of species can be discriminated). Analyses are thus based on species level information unless indicated differently. Where necessary, taxa were assigned names based on translation with a database containing information on fragment lengths per individual species (IS-diagnostics). The procedure consists of two multiplex PCRs: a first PCR had two different fluorescent color labels. One for the phyla Firmicutes, Actinobacteria, Fusobacteria and Verrucomicrobia (FAFV) and a second color for the phylum Bacteroidetes [13]. A separate PCR was performed for the amplification of species of the phylum Proteobacteria. The assay was performed according to the protocol provided by the manufacturer. Amplifications were carried out on a Peltier Thermal Cycler PTC-225 (Bio-rad, California, USA). After PCR, $5 \mu$ l of PCR product was mixed with $20 \mu \mathrm{l}$ formamide and $0.5 \mu$ l Mapmaker 1500 ROX-labeled size marker (BioVentures, Murfreesboro, TN, USA). DNA fragment size analysis was performed on an ABI Prism 3500 Genetic Analyzer (Applied Biosystems).

Data analysis and correlation with metabolites

Pre-processing was carried out by the IS-pro proprietary software suite (ISDiagnostics) and resulted in microbial profiles. Each profile consisted of a set of color-labelled peaks, with each peak related to a specific IS fragment (measured in nucleotides) and the color to a specific phylum group (FAFV, Bacteroidetes or Proteobacteria). The intensity of peaks reflected the quantity of PCR product (measured in relative fluorescent units). As discussed above, a bacterial species is characterized by a specific set of peaks. For simplicity, we considered each peak as an operational taxonomic unit (OTU) and its corresponding intensity as abundance, as these were previously shown to be highly correlated [13,22].

Diversity was calculated per sample as the Shannon index that was recently shown to be a robust estimation of microbial diversity [23]. Dissimilarities between samples were calculated as the cosine distance between each pair of samples. These were summarized and visualized in a low-dimensional space using principal coordinate analysis (PCoA).

Longitudinally differentially abundant OTUs were identified using the function fitTimeSeries, implemented in the metagenomeSeq Bioconductor package [24,25]. This method is intended for estimating and detecting OTUs due to differential abundance of a quantitative measurement. It uses a smoothing spline ANOVA to model longitudinal data while incorporating other covariates in the model (as the diet in our case). This approach was applied twice to compare the microbial profiles of samples of each of the varied diets (high carbohydrate diet and high protein diet) to those of samples with normal diet. Abundance was first normalized 
and $\log 2$ transformed. P-values were calculated and adjusted by false discovery rate (FDR) for each OTU. OTUs at an FDR of 0.05 were considered to be differentially abundant. A clustered heat map was made by generating a cosine correlation matrix of all $\log 2$ transformed profile data followed by clustering with the unweighted pair group method with arithmetic mean (UPGMA).

Spearman correlations were calculated between the abundance of the OTUs that were identified to be differentially abundant between each of the varied diets and the normal diet, and the measured amounts of the metabolites produced.

\section{Ethical approval}

Studies using fecal donations from healthy volunteers do not require medical ethical committee approval in The Netherlands since they are considered as noninvasive. Nevertheless, all participants provided informed consent.

\section{Results}

\section{Diet modulates microbial activity}

Luminal $\mathrm{pH}$

We visualized how fermentations of the diets influenced the luminal $\mathrm{pH}$ in the system by recording the sodium hydroxide $(2 \mathrm{M} \mathrm{NaOH})$ consumption used to titrate the production of SCFA in time (Fig. 1). As mentioned in the description of the TIM-2 system protocol, the luminal $\mathrm{pH}$ is maintained by default at 5.8. However, an indicator of how the $\mathrm{pH}$ might be influenced by the fermentative substrates is by checking the titration system. If the $\mathrm{pH}$ drops below 5.8 more consumption of the $\mathrm{NaOH}$ occurs in order to bring the $\mathrm{pH}$ back to 5.8. Whilst, if the $\mathrm{pH}$ turns higher than 5.8, there is no consumption of $\mathrm{NaOH}$. The normal and the high carbohydrate diets led to more acid production as reflected by the increase in $\mathrm{NaOH}$ use, while an increase in $\mathrm{pH}$ (average of 6.3) was observed in the experiment with the high protein diet when compared to the other diets, which resulted in no $\mathrm{NaOH}$ use after the adaptation period (Fig. 1). These results are in line with those reported in Chapter 4 of this thesis. 


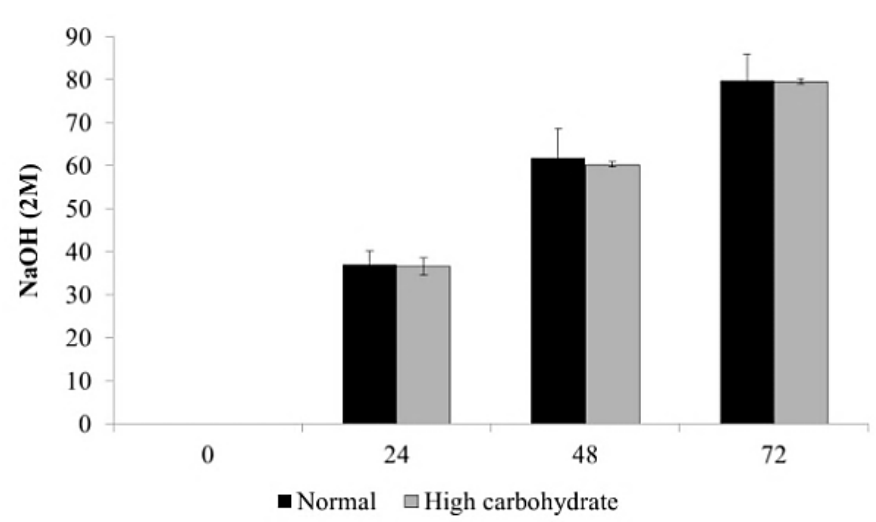

Fig. 1. Cumulative consumption of $\mathrm{NaOH}(2 \mathrm{M})$ used to titrate the system during the $72 \mathrm{~h}$ fermentation with the different diets. Values used by the high protein diet are not depicted since no consumption of $\mathrm{NaOH}$ was observed in this diet. Values were artificially set to zero at the start of feeding the different test diets (t0).

\section{SCFA production}

During the 3 day fermentation of the normal and high carbohydrate diet, the main SCFA produced was acetate followed by $n$-butyrate and propionate (Fig. 2a and 2b, respectively). In the case of the high carbohydrate diet (Fig. 2b), $n$-butyrate and propionate remain lower when compared to the control (Fig. 2a).

In the high protein diet, acetate was still the main product of the fermentation while propionate and $n$-butyrate were similar during the $72 \mathrm{~h}$ experiment (Fig. 2c). The cumulative amounts of acetate and $n$-butyrate were lower than the control and the high carbohydrate diet, as expected. However, the amount of propionate produced was very similar to the other two diets. This corroborates findings in Chapter 4 of this thesis.

The final cumulative amount of total SCFA (Fig. S1) was the highest in the fermentation with the normal diet $(169.4 \mathrm{mmol})$ followed by high carbohydrate and the high protein diet (137.3 and $82.3 \mathrm{mmol}$, respectively). 

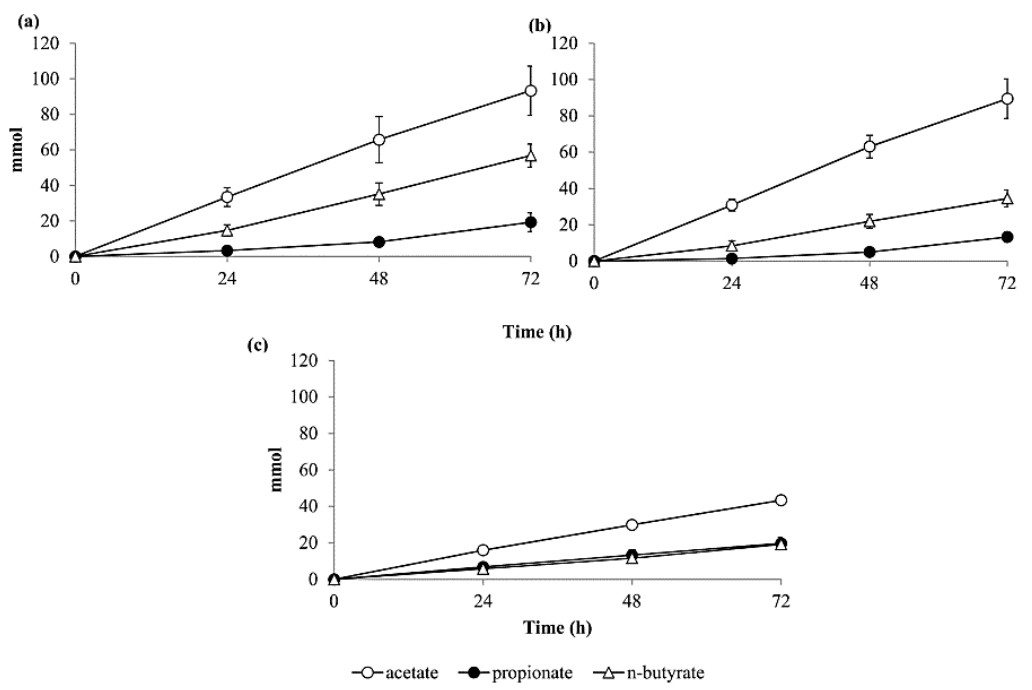

Fig. 2. Average kinetics of cumulative SCFA production (mmol) in the experiments with the (a) normal (control or basal SIEM medium), (b) high carbohydrate and (c) high protein diet. The concentration at t0 was artificially set to zero.

\section{BCFA production}

The amounts for iso-valerate and iso-butyrate started to rise after $48 \mathrm{~h}$ of the experiment with the normal diet whilst their production increased after $24 \mathrm{~h}$ in the high protein diet (Fig. 3). Production of BCFA was below the limit of detection in the experiments with the high carbohydrate diet emphasizing the TIM-2 system as a sensitive tool for the measurement of changes in the gut microbiota activity.

Cumulative iso-valerate production was remarkably higher when compared to isobutyrate in the high protein diet (Fig. S2).

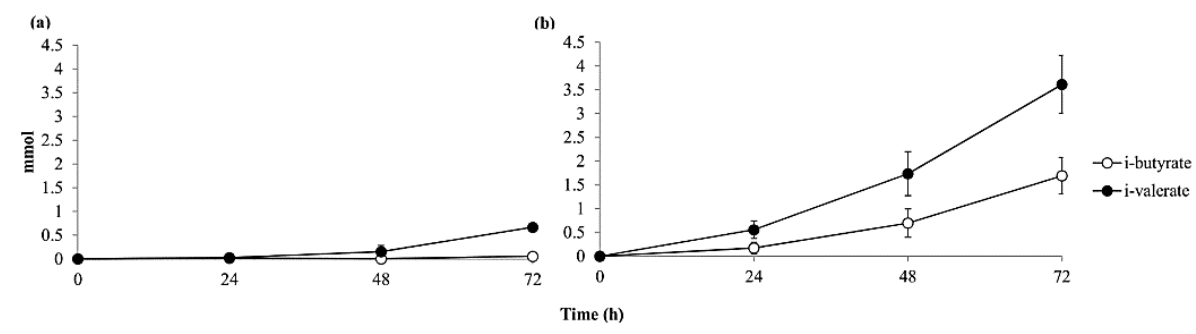

Fig. 3. Average kinetics of cumulative BCFA production (mmol) in the experiments with (a) normal diet, (b) high protein diet. Production of BCFA was below the limit of detection in the experiments with the high carbohydrate diet. The concentration at t0 was artificially set to zero. 


\section{Diet modulates microbial composition}

The analysis on the diversity change over time of each phylum group showed different responses of the communities depending on the diet tested (Fig. 4). In general, the high protein diet showed to be less favorable for FAFV species while it kept a constant diversity value in Proteobacteria species. An increase in diversity in this diet was observed in the Bacteroidetes group after the first $24 \mathrm{~h}$ of the study. However, such increase was less pronounced when compared to the normal and high carbohydrate diet which further increased (t48). Interestingly, when the microbiota was exposed to the normal and high carbohydrate diets, it exhibited a similar change in diversity in all phyla during the study. These diets showed a tendency to keep diversity values constant in FAFV species while for Bacteroidetes and Proteobacteria they tended to increase (t48) and after that stabilized ( $\mathrm{t} 72)$.
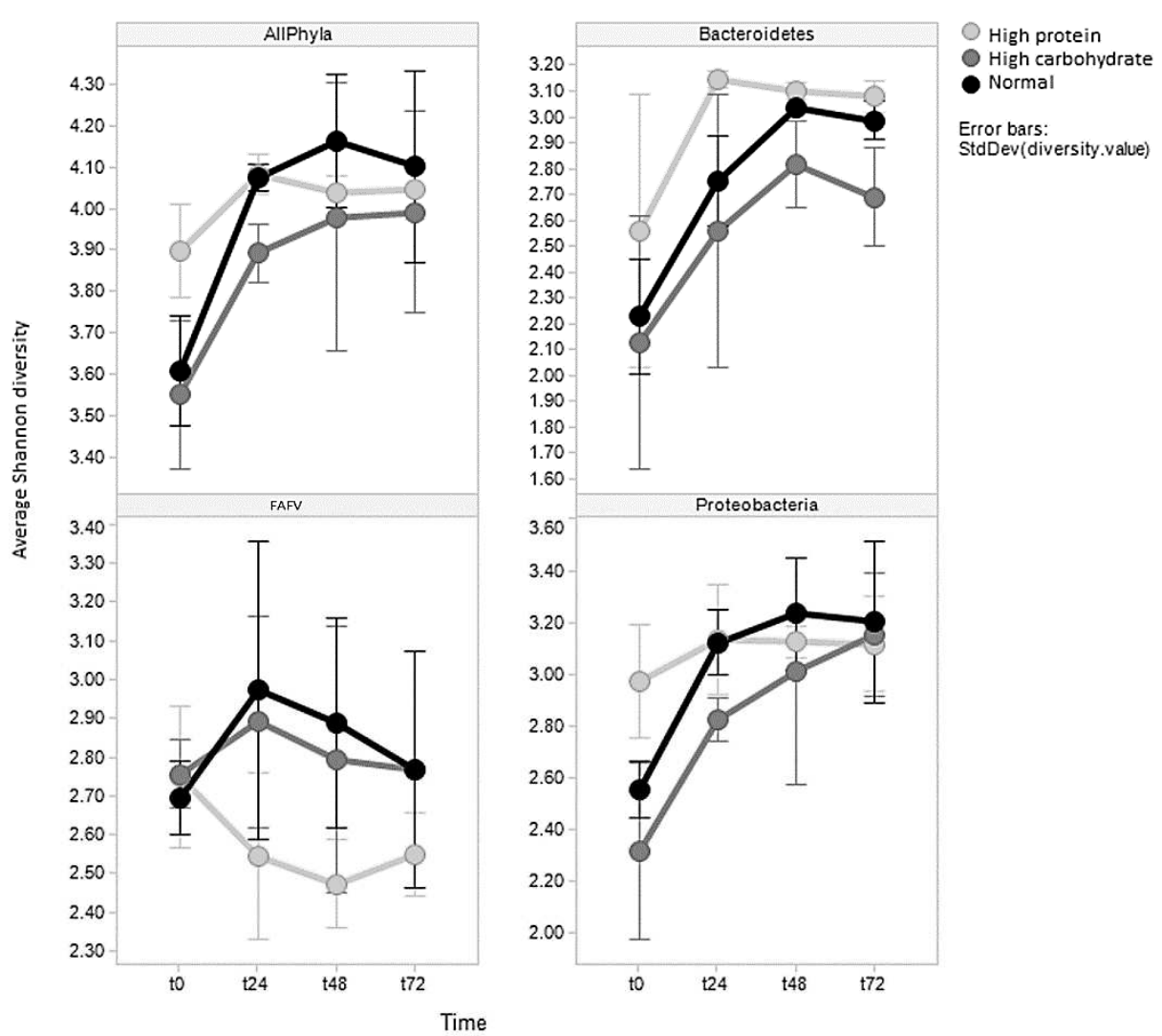

Fig. 4. Phylum specific effect of diet on microbial diversity. Average Shannon diversity index was calculated at each time point per sample and is presented per phylum and for species of all phyla combined. The high protein diet was less favorable for species of the 
FAFV phyla, while the high carbohydrate diet was less favorable for species of the Bacteroidetes and Proteobacteria phyla.

The PCoA in Fig. 5 shows the dissimilarity in microbiome composition in the experimental diets based on cosine distance measures. The analysis displays a clear effect of the diet. Most changes in the microbiome composition occurred during the first $24 \mathrm{~h}$ of the experiment (long distances). After this period, the community seemed to stabilize (short distances). Furthermore, all changes observed in the duplicates were similar which gives a nice indication of the reproducibility of the TIM-2 system.

Zooming in on the effects of the different diets on the composition of the microbiota, a longitudinal analysis that identifies differentially abundant OTUs was applied. When the high carbohydrate and the high protein diets were compared to the normal diet, the set of identified marker OTUs for each of these diets (Fig. 6) showed similar effects as observed for the complete microbiota (Fig 5). Species that were detected both in samples that were fed by the high protein diet and control samples were mostly of the Bacteroidetes phylum, including Bacteroides ovatus and Bacteroides fragilis, while species that were detected both in samples of the high carbohydrate diet and control samples were mostly of the FAFV group, including a Streptococcus species and Eubacterium ventriosum. In the case of Proteobacteria, Escherichia coli was observed to be shared between the high carbohydrate and the normal diet. Based on the set of marker OTUs, samples were clustered into three distinct groups, each representing one of the three diets (Fig. 6a). Within each of these clusters, microbial profiles at 24 clustered separately from profiles of samples taken at $\mathrm{t} 48$ and $\mathrm{t} 72$ indicating that the effect of the diet on these species occurs mostly during the first 24 hours and is later stabilized and confirming the observations from the PCoA analysis (Fig. 5). 


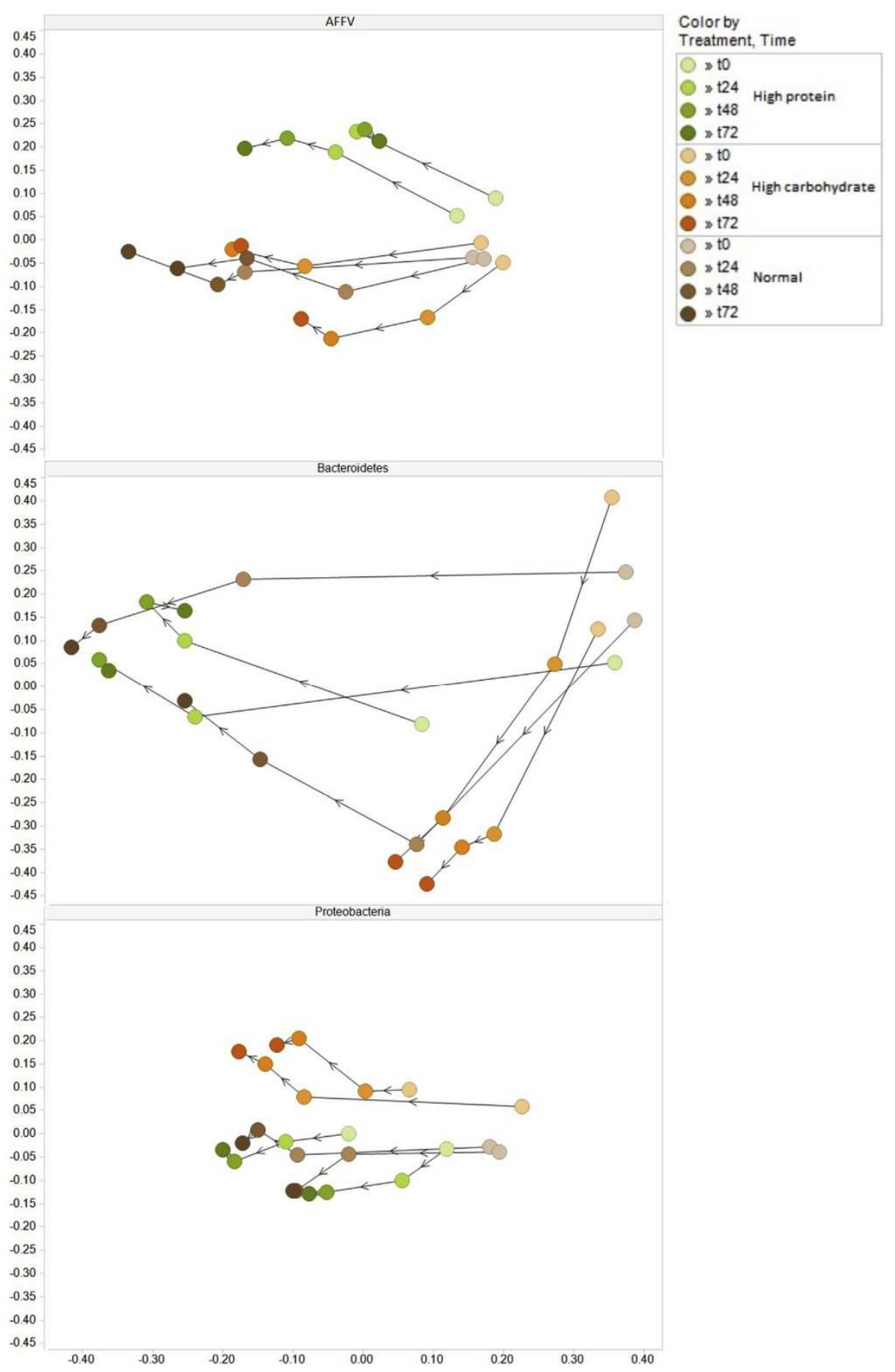

Fig. 5. Phylum specific effect of diet on microbial composition dynamics. Principle coordinate analysis (PCoA) reflects the between-sample diversity and is presented per phylum. A phylumspecific effect of the different diets is observed: Bacteroidetes and 
Proteobacteria species diverged in their response to the high carbohydrate diet compared to the normal diet, while FAFV species diverged in their response to the high protein diet compared to the normal diet. Most changes in the microbial composition occurred during the first $24 \mathrm{~h}$ of the experiment (indicated by longer distances).
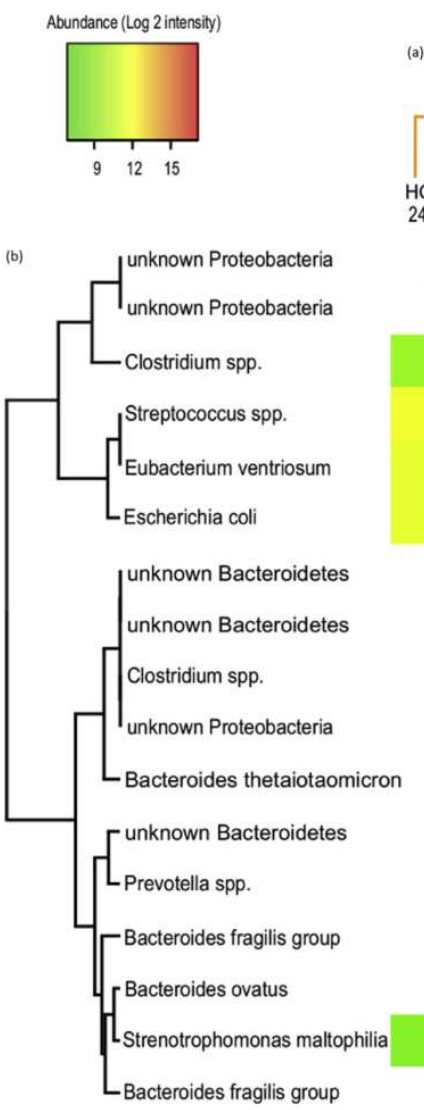
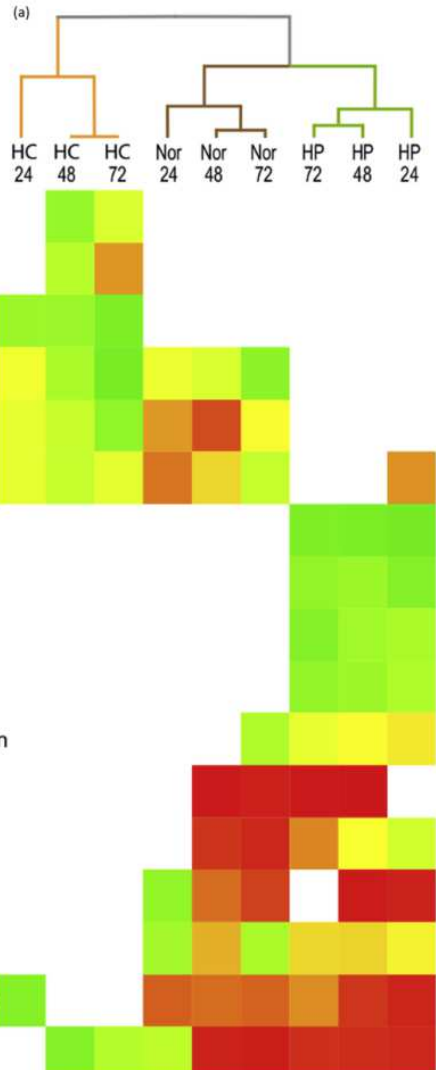

Fig. 6. Samples are clustered according to diet based on a subset of differentially abundant marker species. a) Columns correspond to diet at a certain time point; b) rows correspond to bacterial OTUs identified by IS-pro. Red and green denote relatively high and low averaged abundance of each OTU over the two duplicates, respectively. HC: High carbohydrate diet (orange); Nor: normal diet (brown); HP: high protein diet (green). Time points are indicated at the top of each column. Columns and rows are clustered by cosine correlation. 


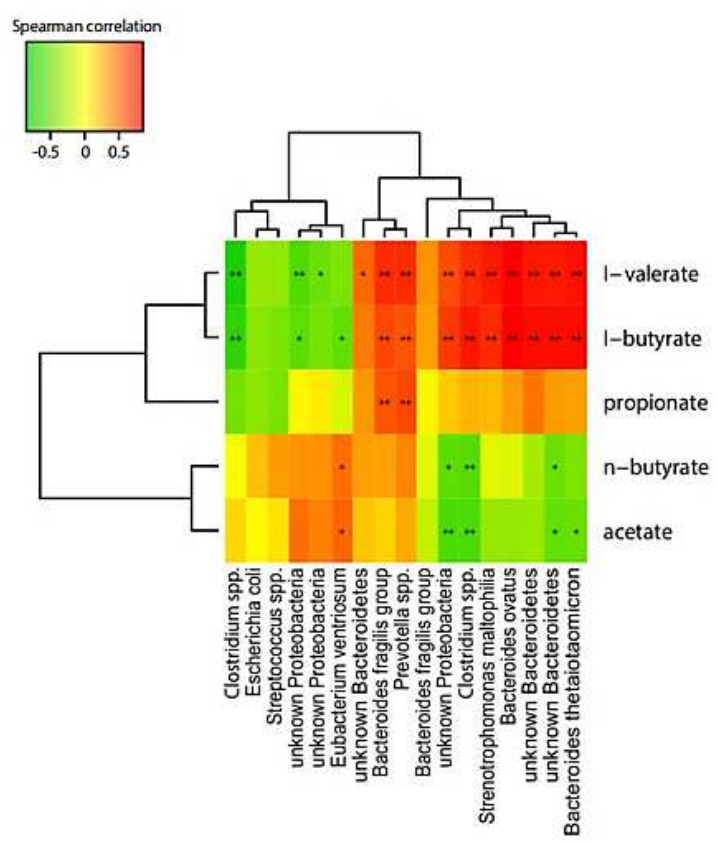

Fig. 7. Correlation of metabolites and a subset of marker bacterial species. Columns correspond to bacterial taxa quantified by IS-pro; rows correspond to measured metabolites. Red and green denote positive and negative correlation, respectively. The intensity of the colors represents the degree of association between the taxa abundances and metabolites as measured by the Spearman's correlations. The ' $*$ ' and ' $* *$ ' indicate the associations that are significant at an FDR of $10 \%$ and 5\%, respectively. Columns and rows are clustered by Euclidean distance.

\section{Diet modulates microbial activity through modulation of its composition}

In correspondence with our results above, Spearman correlations between the main OTUs that where modulated by the diet and the produced metabolites amounts corroborated our findings regarding the modulation of the microbiota activity by the diet (Fig. 7). OTUs in general were clustered into two distinct groups: OTUs that were negatively correlated with propionate and BCFA and OTUs that were positively correlated with BCFA and propionate. OTUs that belong to the former are found in higher abundance in samples of the high carbohydrate diet compared to samples of the high protein diet, in agreement with our findings presented in Fig. 6. OTUs that belong to the latter were found in higher abundance in samples of the high protein diet compared with samples of the high carbohydrate diet. 
More specifically, the correlation analysis performed showed a significant correlation of E. ventriosum, Clostridium spp., an unknown Bacteroidetes and an unknown Proteobacteria with acetate and $n$-butyrate production; propionate production was significantly correlated with Bacteroides and Prevotella species; BCFA were mostly correlated with Bacteroidetes and Proteobacteria OTUs (Fig. $7)$.

\section{Discussion}

Designing carefully controlled, large-scale human trials investigating the impact of diet on gut microbiota composition and activity have several limitations [26]. Ethical and social considerations [27] restrict the execution of invasive human studies. Besides, it is difficult to guarantee the strict compliance to diets of participants, and their imprecision in self-reporting data has to be considered [28]. Moreover, metabolite measurements in feces do not reflect the activity of the microbiota along the entire large intestine, and hence may give a skewed picture. Additionally, these types of studies fail to properly establish the mechanisms behind changes in the gut community and their promotion of disease. A solution to these limitations is performing carefully controlled in vitro studies in validated models. In such studies samples are assessed by innovative technological tools such as cell models, microarrays, sequencing and profiling platforms. This provides helpful insight to answer fundamental still unanswered questions regarding: i) the potential of certain dietary compounds in promoting a specific microbial composition and/or activity; ii) the role of certain bacteria in the treatment/prevention of diseases.

The aim of this study was to screen how fast changes in bacterial activity and composition can be induced when major modifications in carbohydrate and protein content were supplied in the diet provided to the microbiota.

The detectable differences when diet is changed in a human individual have not been fully explored and it is unclear how fast and reproducibly the microbiota can respond to these changes $[6,29]$. However, one of the few studies in this area was performed by David et al. [6], who nicely demonstrated that the consumption of a diet, mainly composed either from plant or animal products, modifies the composition and the activity of the gut microbiota of healthy subjects in only five days. The authors found that even only one day after initiating the study, there was an increase in $\beta$-diversity in the microbiota from the feces of the individuals following the animal diet and that their original structure recovered after two days of finishing with the study. Furthermore, a higher concentration of products 
associated with amino acid fermentation was found in the animal based diet consumers when compared to the plant based one.

Although high levels of fat are most of the time associated with unhealthy diets (e.g. Western diet [30]) and hence link it with different diseases, it is unclear how much fat makes it to the colon. Some initial studies have provided some insight in this and indicate that from a load of $70 \mathrm{~g} / \mathrm{day} 3.5 \mathrm{~g}$ of fat reaches the colon [31]. Nonetheless, healthy and unhealthy diets have been also associated with differences in fiber, protein and sugar content, providing nutritional choices (other than fat) to focus on with in vitro studies related to diets and microbiota [32]. In general, healthy diets have been associated with the consumption of foods with the adequate essential nutrition supporting energy needs, while unhealthy diets have been characterized by the consumption of high energy-dense foods rich in sugar or saturated fat with a poor nutritional quality [33]. The current study provided valuable insight into the ability of the gut microbiota to respond and adapt to different protein/carbohydrate ratios in the medium, which consequently suggests what could happen under in vivo conditions.

\section{Diet modulates microbial activity}

The activity of the microbiota reflected differences between the high protein and the high carbohydrate diet exhibiting a trade-off between saccharolytic and proteolytic fermentation when compared to the normal diet (Fig. 2 and 3). This is confirmed by the different cumulative total amounts and ratios of SCFA and BCFA (Fig. S1 and S2) observed in each diet. Furthermore, despite the fact that the experiments were maintained at a $\mathrm{pH}$ of 5.8 to mimic the conditions in the proximal colon [16], differences were also observed in the amount of $\mathrm{NaOH}$ used to titrate the production of acids in the lumen (Fig. 1). All these observations were found after the first $24 \mathrm{~h}$ of experimenting with the diets, though the differences were accentuated through time.

The $\mathrm{pH}$ in the proximal colon has been found to be slightly acidic due to the production of SCFA from the degradation of carbohydrates by bacterial exoenzymes and subsequent fermentation of liberated sugars and oligosaccharides $[34,35]$. As fermentable carbohydrates become depleted, protein fermentation and $\mathrm{pH}$ increase in the distal colon. Correspondingly, a high fecal $\mathrm{pH}$ has been found in subjects following a high animal protein diet [36]. Therefore, an adequate supply of fermentable carbohydrates has been suggested to help to maintain an optimal (acidic) $\mathrm{pH}$ in the colon [7]. Lowering the $\mathrm{pH}$ is a strategy to improve gut health in humans as it has been shown to inhibit the growth of groups of pathogenic bacteria including E. coli and other Enterobacteriaceae [36,37]. Moreover, an increase of carbohydrate consumption has been suggested as a means to limit the proteolytic 
fermentation [38] the products of which are considered detrimental to the host due to their toxicity [18].

In contrast to metabolites produced from protein fermentation, the physiological effects of acetate, propionate and butyrate, have been extensively reviewed due to their contribution to energy balance and their particular significance in maintaining gut health [39-41]. These SCFA are associated with carbohydrate fermentation although their production has been also shown from many amino acids [42]. Effects of butyrate as an anti-inflammatory and anti-carcinogenic molecule have been well documented $[34,43]$ whilst acetate and propionate have been found to be involved in supporting de novo lipid synthesis in the liver and as a substrate in gluconeogenesis, respectively [34]. As mentioned before, products from proteolytic fermentation include SCFA. However, BCFA, which are principally iso-butyrate, iso-valerate and 2-methylbutyrate, exclusively originate from proteolytic fermentation [35]. Therefore, their excretion is normally used as a marker to evaluate the level of fermentation of proteins in the colon [35]. In this study, as expected, the production of BCFA was inhibited by a diet high in fermentable carbohydrates. Furthermore, it was possible to detect the metabolic response from the microbiota already after the first $24 \mathrm{~h}$ of experimenting with the diets. Such a fast effect suggests, once again, that the manipulation of the metabolic activity of the microbiota via dietary means can be achieved in order to counteract the negative effects of certain dietary components characteristic from a western diet such as elevated amounts of protein.

It has been suggested that a prolonged exposure to BCFA and other products from fermentation of proteins, such as ammonia, phenol, $p$-cresol, biogenic amines and hydrogen-sulfide may principally affect the viability of colonic epithelial cells [44], promote genomic DNA damage [45] and increase colonic adenocarcinomas [46]. Also systemically, these metabolites have been reported to have detrimental effects. Still, proteolytic fermentation is less studied when compared to carbohydrates [34]. Therefore, further research is required that focuses on the production of metabolites from protein fermentation.

\section{Diet modulates microbial composition}

In this study we observed changes in the diversity of the microbiota (Fig. 4) dependent on the availability of the fermentable substrate. For instance, the high protein diet decreased the diversity of species of the Firmicutes phylum which have been found to metabolize dietary plant polysaccharides [6] and increased the diversity of Bacteroidetes species (Fig. 4; Fig. 6). Such changes were already found after the first $24 \mathrm{~h}$ of providing the diets to the microbiota. 
It has been observed that several proteolytic bacteria characterized from human feces belong to the Bacteroides genus, of which peptidase activity is considered to be high [38]. Furthermore, our findings indicating the elevated abundance of certain Bacteroidetes species in the high protein diet when compared to the high carbohydrate diet (Fig. 6) are also in agreement with Maier et al. [47] and Zimmer et al. [36]. These authors observed a high abundance of Bacteroides species in subjects who followed a high protein diet [47] but lower counts in subjects following a vegetarian and vegan diet [36].

Bacteroides species including B. thetaiotaomicron, B. fragilis and B.ovatus were found to be differentially abundant between the diets (Fig. 6b). Though many studies have focused on the growth of B. thetaiotaomicron from polysaccharide fermentation, this organism has been considered as metabolically flexible. It has been observed that $B$. thetaiotaomicron can direct its metabolism from host-derived (in particular mucus, a gel composed mainly of water and glycoproteins) to dietary polysaccharides according to nutrient availability [48]. With respect to B. fragilis, lower abundance was observed in obese subjects when compared to lean subjects [49] suggesting that this microorganism could be associated with an healthy diet. Interestingly, $B$. ovatus has been found to be involved in the production of phenylpropanoid-derived metabolites [50] suggesting its ability to ferment proteins, which is confirmed in this study.

Identifying the most discriminative species also provided evidence about the rapid response of the microbiota to diet. From the samples' clustering, based on a selected subset of differentially abundant species (Fig. 6a), we can infer that the effect of diet on the microbiota occurs in the first $24 \mathrm{~h}$, as samples at this time point cluster separately from samples taken at $48 \mathrm{~h}$ and $72 \mathrm{~h}$.

We acknowledge the limitations of working with an in vitro system for fermentation studies. Despite that in these experiments the operational conditions are rigorously controlled and standardized, the microbiota is often exposed to factors (for instance, redox potential, osmotic stress, temperature) that affect the development of the community after an adaptation period to the system (from human gut to in vitro model) [51]. Therefore, some OTUs were observed to be highly present at 0 , that is: before the intervention with the diets. This highlights the importance of including a control which in this case is the normal diet which is used as a reference for measuring the impact of diet on the microbiota.

\section{Diet modulates microbial activity through modulation of its composition}

The ratio for propionate production in the high protein diet was higher when compared to the other diets (Fig. S1). Furthermore, this metabolite showed a significant correlation with Bacteroides and Prevotella species (Fig. 7). This is in 
line with the fact that Bacteroidetes has been considered as the largest group in the gut microbiota producing propionate [52,53]. Another interesting finding from this study is the high ratio of $n$-butyrate produced in the normal diet (Fig. S1), which could be explained by the high abundance of E. ventriosum, a known butyrate producer [54] (Fig. 6b) and its positive correlation with this metabolite (Fig. 7).

As also observed by Durban et al. [55] the reduction of carbohydrate content in the diet caused an effect of instability in the diversity of the community as reflected by the larger variation in the diversity values. Furthermore, these authors also observed similarities in the production of propionate despite the differences in the nutrient load provided to the microbiota.

Importantly, and confirming our hypothesis, this study showed a very short timedependent response of the gut microbiota, both in composition and activity, after being exposed to different diets (Fig. 5). In general, substantial changes occurred during the first $24 \mathrm{~h}$ after providing the different diets. After this initial period, the community tended to stabilize.

We conclude that the intentional modulation of human gut microbiota is possible using a dietary approach. This study confirms a fast response (metabolically and compositionally) of the gut microbiota to diet. However, inter-individual differences have to be considered for a full assessment of the impact of certain dietary compounds on the microbiota. Therefore, future studies, including in vivo trials, are suggested to explore the similarities in the response of diet in different individuals.

\section{Conflict of interest}

The authors declare that there are no conflicts of interest.

\section{Acknowledgments}

The authors thank Malieka van der Lugt-Degen for assisting during the ABI runs and Carel Peeters for helpful statistical advice. This study was partly funded by the Top Institute Food \& Nutrition (TIFN, Wageningen, The Netherlands). 
Diet fermentation in TIM-2

\section{References}

1. Aguirre M, Venema K (2015) Does the Gut Microbiota Contribute to Obesity? Going beyond the Gut Feeling. Microorganisms 3: 213-235.

2. Vipperla K, O'Keefe SJ (2012) The microbiota and its metabolites in colonic mucosal health and cancer risk. Nutr Clin Pract 27: 624-635.

3. Koeth RA, Wang Z, Levison BS, Buffa JA, Org E, et al. (2013) Intestinal microbiota metabolism of 1-carnitine, a nutrient in red meat, promotes atherosclerosis. Nat Med 19: 576-585.

4. Cani PD, Amar J, Iglesias MA, Poggi M, Knauf C, et al. (2007) Metabolic Endotoxemia Initiates Obesity and Insulin Resistance. Diabetes 56: 17611772.

5. Wu GD, Chen J, Hoffmann C, Bittinger K, Chen Y-Y, et al. (2011) Linking LongTerm Dietary Patterns with Gut Microbial Enterotypes. Science 334: 105-108.

6. David LA, Maurice CF, Carmody RN, Gootenberg DB, Button JE, et al. (2014) Diet rapidly and reproducibly alters the human gut microbiome. Nature 505: 559563.

7. Duncan SH, Belenguer A, Holtrop G, Johnstone AM, Flint HJ, et al. (2007) Reduced Dietary Intake of Carbohydrates by Obese Subjects Results in Decreased Concentrations of Butyrate and Butyrate-Producing Bacteria in Feces. Appl Environ Microbiol 73: 1073-1078.

8. Aguirre M, Jonkers DM, Troost FJ, Roeselers G, Venema K (2014) In vitro characterization of the impact of different substrates on metabolite production, energy extraction and composition of gut microbiota from lean and obese subjects. PLoS One 9: e113864.

9. Bussolo de Souza C, Roeselers G, Troost F, Jonkers D, Koenen ME, et al. (2014) Prebiotic effects of cassava bagasse in TNO's in vitro model of the colon in lean versus obese microbiota. J Funct Foods 11: 210-220.

10. Dao MC, Everard A, Aron-Wisnewsky J, Sokolovska N, Prifti E, et al. (2015) Akkermansia muciniphila and improved metabolic health during a dietary intervention in obesity: relationship with gut microbiome richness and ecology. Gut 65: 426-436.

11. Seo DB, Jeong HW, Cho D, Lee BJ, Lee JH, et al. (2015) Fermented green tea extract alleviates obesity and related complications and alters gut microbiota composition in diet-induced obese mice. J Med Food 18: 549-556.

12. Minekus M, Smeets-Peeters M, Bernalier A, Marol-Bonnin S, Havenaar R, et al. (1999) A computer-controlled system to simulate conditions of the large intestine with peristaltic mixing, water absorption and absorption of fermentation products. Appl Microbiol Biotechnol 53: 108-114. 
13. Budding AE, Grasman ME, Lin F, Bogaards JA, Soeltan-Kaersenhout DJ, et al. (2010) IS-pro: high-throughput molecular fingerprinting of the intestinal microbiota. FASEB J 24: 4556-4564.

14. Venema K, Van Nuenen HMC, Smeets-Peeters M, Minekus M, Havenaar R (2000) TNO's in vitro large intestinal model: an excellent screening tool for functional food and pharmaceutical research. Ernährung/Nutrition 24: 558564.

15. Rajilic-Stojanovic M, Maathuis A, Heilig HG, Venema K, de Vos WM, et al. (2010) Evaluating the microbial diversity of an in vitro model of the human large intestine by phylogenetic microarray analysis. Microbiology 156: 32703281.

16. Maathuis A, Hoffman A, Evans A, Sanders L, Venema K (2009) The Effect of the Undigested Fraction of Maize Products on the Activity and Composition of the Microbiota Determined in a Dynamic in Vitro Model of the Human Proximal Large Intestine. J Am Coll Nutr 28: 657-666.

17. Maathuis AJ, van den Heuvel EG, Schoterman MH, Venema K (2012) Galactooligosaccharides have prebiotic activity in a dynamic in vitro colon model using a (13)C-labeling technique. J Nutr 142: 1205-1212.

18. Van Nuenen HMC, Meyer PD, Venema K (2003) The effect of various inulins and Clostridium difficile on the metabolic activity of the human colonic microbiota in vitro. Microb Ecol Health Dis 15: 137-144.

19. De Graaf AA, Maathuis A, De Waard P, Deutz NEP, Dijkema C, et al. (2010) Profiling human gut bacterial metabolism and its kinetics using [U-13C] glucose and NMR NMR in Biomedicine 23: 2-12.

20. Rose DJ, Venema K, Keshavarzian A, Hamaker BR (2010) Starch-entrapped microspheres show a beneficial fermentation profile and decrease in potentially harmful bacteria during in vitro fermentation in faecal microbiota obtained from patients with inflammatory bowel disease. Br J Nutr 103: 15141524.

21. Van den Abbeele P, Venema K, Van de Wiele T, Verstraete W, Possemiers S (2013) Different Human Gut Models Reveal the Distinct Fermentation Patterns of Arabinoxylan versus Inulin. J Agric Food Chem 61: 9819-9827.

22. Budding AE, Vandenbroucke-Grauls CM, Melles DC, van Duijkeren E, Kluytmans JA, et al. (2010) Binary IS typing for Staphylococcus aureus. PLoS One 5: e13671.

23. Haegeman B, Hamelin J, Moriarty J, Neal P, Dushoff J, et al. (2013) Robust estimation of microbial diversity in theory and in practice. ISME $J$ 7: 10921101.

24. Paulson JN, Stine OC, Bravo HC, Pop M (2013) Differential abundance analysis for microbial marker-gene surveys. Nat Methods 10: 1200-1202. 
25. Paulson JN, Pop M, Bravo HC metagenomeSeq: Statistical analysis for sparse high-throughput sequencing. Bioconductor package: 1.10.0.cbcb.umd.edu/software/metagenomeSeq.

26. Power SE, O'Toole PW, Stanton C, Ross RP, Fitzgerald GF (2014) Intestinal microbiota, diet and health. Br J Nutr 111: 387-402.

27. Payne AN, Zihler A, Chassard C, Lacroix C (2012) Advances and perspectives in in vitro human gut fermentation modeling. Trends Biotechnol 30: 17-25.

28. Poslusna K, Ruprich J, de Vries JH, Jakubikova M, van't Veer P (2009) Misreporting of energy and micronutrient intake estimated by food records and 24 hour recalls, control and adjustment methods in practice. $\mathrm{Br} J$ Nutr 101 Suppl 2: S73-85.

29. Muegge BD, Kuczynski J, Knights D, Clemente JC, Gonzalez A, et al. (2011) Diet drives convergence in gut microbiome functions across mammalian phylogeny and within humans. Science 332: 970-974.

30. Cordain L, Eaton SB, Sebastian A, Mann N, Lindeberg S, et al. (2005) Origins and evolution of the Western diet: health implications for the 21 st century. Am $J$ Clin Nutr 81: 341-354.

31. Priebe MG, Vonk RJ, Sun X, He T, Harmsen HJ, et al. (2002) The physiology of colonic metabolism. Possibilities for interventions with pre- and probiotics. Eur J Nutr 41 Suppl 1: I2-10.

32. Payne AN, Chassard C, Banz Y, Lacroix C (2012) The composition and metabolic activity of child gut microbiota demonstrate differential adaptation to varied nutrient loads in an in vitro model of colonic fermentation. FEMS Microbiol Ecol 80: 608-623.

33. De Vet E, Stok FM, De Wit JB, De Ridder DT (2015) The habitual nature of unhealthy snacking: How powerful are habits in adolescence? Appetite 95: $182-187$.

34. Salonen A, de Vos WM (2014) Impact of diet on human intestinal microbiota and health. Anпи Rev Food Sci Technol 5: 239-262.

35. Windey K, De Preter V, Verbeke K (2012) Relevance of protein fermentation to gut health. Mol Nutr Food Res 56: 184-196.

36. Zimmer J, Lange B, Frick JS, Sauer H, Zimmermann K, et al. (2012) A vegan or vegetarian diet substantially alters the human colonic faecal microbiota. Eur $J$ Clin Nutr 66: 53-60.

37. De Preter V, Vanhoutte T, Huys G, Swings J, De Vuyst L, et al. (2007) Effects of Lactobacillus casei Shirota, Bifidobacterium breve, and oligofructose-enriched inulin on colonic nitrogen-protein metabolism in healthy humans. Am $J$ Physiol Gastrointest Liver Physiol 292: G358-368.

38. Scott KP, Gratz SW, Sheridan PO, Flint HJ, Duncan SH (2013) The influence of diet on the gut microbiota. Pharmacol Res 69: 52-60. 
39. Lin HV, Frassetto A, Kowalik EJ, Jr., Nawrocki AR, Lu MM, et al. (2012) Butyrate and propionate protect against diet-induced obesity and regulate gut hormones via free fatty acid receptor 3-independent mechanisms. PLoS One 7: e35240.

40. Inoue D, Tsujimoto G, Kimura I (2014) Regulation of Energy Homeostasis by GPR41. Front Endocrinol (Lausanne) 5: 81.

41. Canfora EE, Jocken JW, Blaak EE (2015) Short-chain fatty acids in control of body weight and insulin sensitivity. Nat Rev Endocrinol.

42. Blachier F, Mariotti F, Huneau JF, Tomé D (2007) Effects of amino acid-derived luminal metabolites on the colonic epithelium and physiopathological consequences. Amino Acids 33: 547-562.

43. Hamer HM, Jonkers D, Venema K, Vanhoutvin S, Troost FJ, et al. (2008) Review article: the role of butyrate on colonic function. Aliment Pharmacol Ther 27: 104-119.

44. Hughes R, Kurth MJ, McGilligan V, McGlynn H, Rowland I (2008) Effect of colonic bacterial metabolites on Caco-2 cell paracellular permeability in vitro. Nutr Cancer 60: 259-266.

45. Cerini C, Dou L, Anfosso F, Sabatier F, Moal V, et al. (2004) P-cresol, a uremic retention solute, alters the endothelial barrier function in vitro. Thromb Haemost 92: 140-150.

46. Clinton SK, Bostwick DG, Olson LM, Mangian HJ, Visek WJ (1988) Effects of ammonium acetate and sodium cholate on N-methyl-N'-nitro-Nnitrosoguanidine-induced colon carcinogenesis of rats. Cancer Res 48: 30353039.

47. Maier BR, Flynn MA, Burton GC, Tsutakawa RK, Hentges DJ (1974) Effects of a high-beef diet on bowel flora: a preliminary report. Am J Clin Nutr 27: 14701474.

48. Wrzosek L, Miquel S, Noordine ML, Bouet S, Joncquel Chevalier-Curt M, et al. (2013) Bacteroides thetaiotaomicron and Faecalibacterium prausnitzii influence the production of mucus glycans and the development of goblet cells in the colonic epithelium of a gnotobiotic model rodent. BMC Biol 11: 61.

49. Turnbaugh PJ, Ley RE, Mahowald MA, Magrini V, Mardis ER, et al. (2006) An obesity-associated gut microbiome with increased capacity for energy harvest. Nature 444: 1027-1031.

50. Russell WR, Duncan SH, Scobbie L, Duncan G, Cantlay L, et al. (2013) Major phenylpropanoid-derived metabolites in the human gut can arise from microbial fermentation of protein. Mol Nutr Food Res 57: 523-535.

51. Aguirre M, Eck A, Koenen ME, Savelkoul PHM, Budding AE, et al. (2015) Evaluation of an optimal preparation of human standardized fecal inocula for in vitro fermentation studies. J Microbiol Meth 117: 78-84. 
52. Salonen A, Lahti L, Salojarvi J, Holtrop G, Korpela K, et al. (2014) Impact of diet and individual variation on intestinal microbiota composition and fermentation products in obese men. ISME J 8: 2218-2230.

53. Flint HJ, Scott KP, Louis P, Duncan SH (2012) The role of the gut microbiota in nutrition and health. Nat Rev Gastroenterol Hepatol 9: 577-589.

54. Barcenilla A, Pryde SE, Martin JC, Duncan SH, Stewart CS, et al. (2000) Phylogenetic relationships of butyrate-producing bacteria from the human gut. Appl Environ Microbiol 66: 1654-1661.

55. Durbán A, Abellán J, Latorre A, Moya1 A (2013) Effect of Dietary Carbohydrate Restriction on an Obesity-Related Prevotella-Dominated Human Fecal Microbiota. Metagenomics 2: 4. 


\section{Supplemental material}

Table S1. List of OTUs that were identified as differentially abundant between each of the varied diets (high protein diet and high carbohydrate diet) and the control diet, including P-values adjusted by false discovery rate (FDR) for each OTU.

\begin{tabular}{|c|c|c|c|c|c|c|}
\hline Diet & Phyla & Spp. & $\begin{array}{c}\text { Start } \\
\text { time } \\
\text { point }\end{array}$ & $\begin{array}{l}\text { End } \\
\text { time } \\
\text { Point }\end{array}$ & $\begin{array}{c}\text { Pvals } \\
\text { org }\end{array}$ & $\begin{array}{c}\text { Pvals } \\
\text { adjusted }\end{array}$ \\
\hline \multirow{10}{*}{ 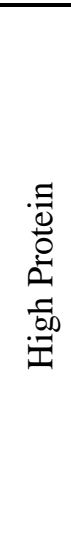 } & \multirow{3}{*}{ 疍 } & Clostridium spp. & 2 & 4 & 0 & 0 \\
\hline & & Streptococcus spp. & 2 & 4 & 0 & 0 \\
\hline & & Eubacterium ventriosum & 2 & 4 & 0 & 0 \\
\hline & \multirow{5}{*}{ 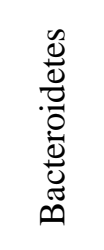 } & Bacteroides & & & & \\
\hline & & thetaiotaomicron & 2 & 4 & 0 & 0 \\
\hline & & unknown Bacteroidetes & 2 & 4 & 0.002 & 0.010 \\
\hline & & unknown Bacteroidetes & 2 & 4 & 0 & 0 \\
\hline & & Prevotella spp. & 2 & 4 & 0.002 & 0.010 \\
\hline & \multirow{2}{*}{$\begin{array}{l}\dot{8} \\
\stackrel{0}{0} \\
\dot{0}\end{array}$} & unknown Proteobacteria & 2 & 4 & 0.003 & 0.013 \\
\hline & & Escherichia coli & 3 & 4 & 0.005 & 0.019 \\
\hline \multirow{13}{*}{ 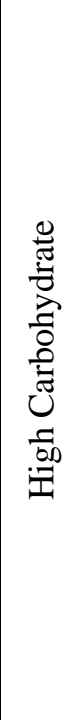 } & 空 & Clostridium spp. & 2 & 4 & 0 & 0 \\
\hline & \multirow{8}{*}{ 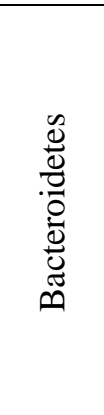 } & unknown Bacteroidetes & 3 & 4 & 0.01 & 0.045 \\
\hline & & Clostridium spp. & 2 & 4 & 0 & 0 \\
\hline & & Bacteroides fragilis group & 2 & 4 & 0.001 & 0.011 \\
\hline & & Prevotella spp. & 3 & 4 & 0.001 & 0.011 \\
\hline & & Prevotella spp. & 3 & 4 & 0.004 & 0.030 \\
\hline & & Bacteroides ovatus & 3 & 4 & 0.012 & 0.049 \\
\hline & & Bacteroides ovatus & 2 & 4 & 0.008 & 0.040 \\
\hline & & Bacteroides fragilis group & 2 & 4 & 0.006 & 0.034 \\
\hline & \multirow{4}{*}{$\begin{array}{l}\dot{0} \\
\stackrel{0}{0} \\
\dot{0}\end{array}$} & unknown Proteobacteria & 3 & 4 & 0.005 & 0.032 \\
\hline & & Strenotrophomonas & & & & \\
\hline & & maltophilia & 3 & 4 & 0.001 & 0.011 \\
\hline & & unknown Proteobacteria & 2 & 4 & 0.003 & 0.027 \\
\hline
\end{tabular}




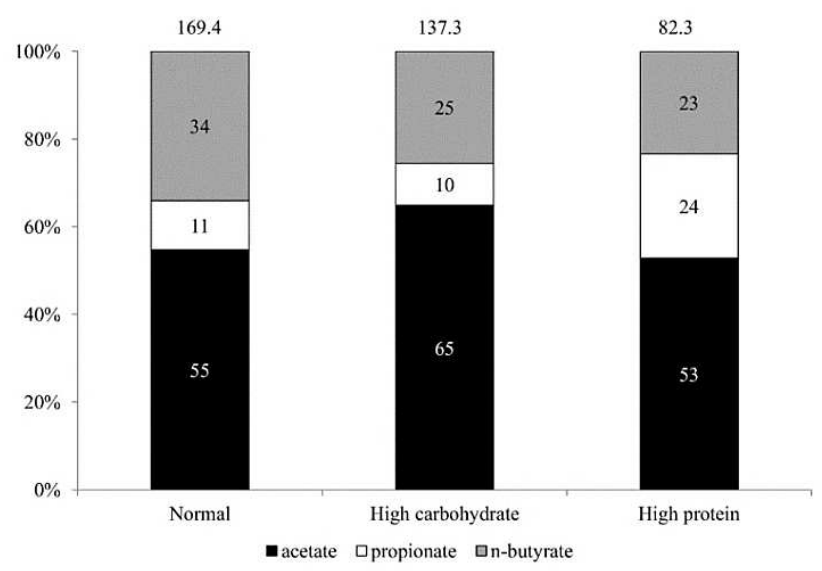

Fig. S1. SCFA ratios (\%) from the different diets at t72. Absolute total SCFA levels are indicated above each bar (mmol).

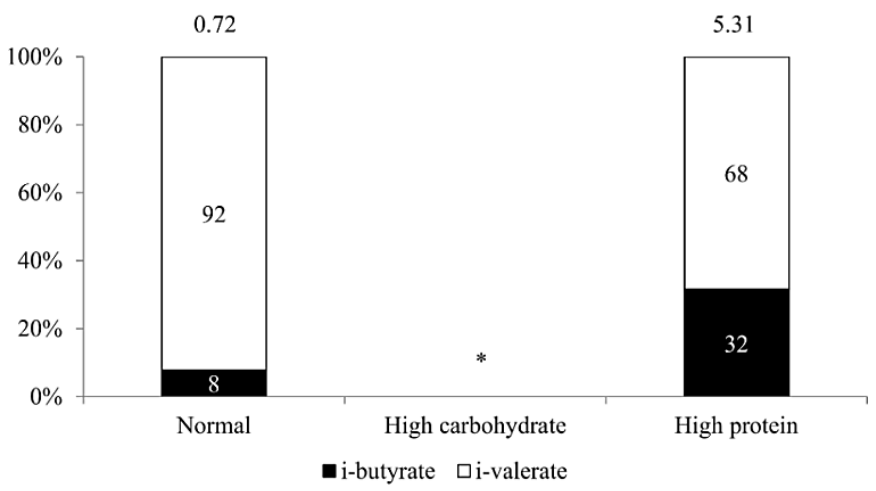

Fig. S2. BCFA ratios (\%) from the different diets at t72. *: the levels measured in the high carbohydrate experiments were below the level of detection. Absolute total BCFA levels are indicated above each bar (mmol). 
Chapter 5 


\title{
Chapter 6
}

\author{
In vitro characterization of the impact of \\ different substrates on metabolite \\ production, energy extraction and \\ composition of gut microbiota from lean and \\ obese subjects
}

Published as:

Aguirre M, Jonkers DMAE, Troost FJ, Roeselers G, Venema K (2014) In Vitro Characterization of the Impact of Different Substrates on Metabolite Production, Energy Extraction and Composition of Gut Microbiota from Lean and Obese Subjects. PLOS ONE 9(11): e113864. 


\section{Abstract}

The aim of this study was to investigate the effect of galacto-oligosaccharides, lactulose, apple fiber and sugar beet pectin on the composition and activity of human colonic microbiota of lean and obese healthy subjects using an in vitro model of the proximal colon: TIM-2

Substrate fermentation was assessed by measuring the production of short-chain and branched-chain fatty acids, lactate and ammonia and by studying the composition of the bacterial communities over time. The results suggest that energy harvest (in terms of metabolites) of lean and obese microbiotas is different and may depend on the fermentable substrate. For galacto-oligosaccharides and lactulose, the cumulative amount of short-chain fatty acids plus lactate produced in TIM-2 was lower in the fermentation experiments with the lean microbiota (-L) (123 and $155 \mathrm{mmol}$, respectively) compared to the obese (-O) (162 and $173 \mathrm{mmol}$, respectively). This was reversed for the pectin and the fiber. The absolute amount produced of short-chain fatty acids including lactate was higher after $72 \mathrm{~h}$ in the fermentation experiments with apple fiber-L $(108 \mathrm{mmol})$ than with apple fiber-O (92 mmol). Sugar beet-L was also higher $(130 \mathrm{mmol})$ compared to sugar beet-O (103 mmol).

Compositionally, Firmicutes were more predominant in the microbiota prepared from feces of obese subjects compared to lean subjects. The average abundance at time zero was $92 \pm 3.3 \%(n=10)$ and $74 \pm 1.0 \%(n=11)$, respectively. On the other hand, Bacteroidetes were more dominant in the microbiota prepared with homogenates from lean subjects with an average abundance of $22 \pm 0.9 \%$ compared with the microbiota prepared with homogenates from obese subjects (3.6 $\pm 1.9 \%$ ). Several groups of bacteria showed changes in their relative abundance. Bifidobacterium was increased in the lean and obese microbiota fermenting galacto-oligosaccharides (600 fold and 100 fold, respectively for lean and obese) and lactulose (4338 and 890 fold). The growth of Catenibacterium spp., Dorea spp., Clostridium cluster XIVb spp. and Faecalibacterium spp. were most stimulated when fermenting apple fiber-L ( 215 fold), apple fiber-O (46 fold), sugar beet-L ( 21 fold) and sugar beet-O ( 8 fold), respectively.

This study provides evidence that different fermentable carbohydrates are fermented differently by lean and obese microbiotas, which contributes to the understanding of the role of diet and the microbiota in tackling obesity. 
Lean vs Obese: apple fiber, sugar beet, GOS, lactulose fermentation

\section{Introduction}

Overweight and obesity constitute two of the main risk factors for a wide range of chronic disorders, including diabetes (with a 44\% increased risk in obese individuals), cardiovascular disease (23\%), high blood pressure and cancer (7$41 \%)[1,2]$.

A complex set of interactions have been unraveled between the colonic microbiota and humans, including a role in overweight and obesity. Studies with germ-free mice demonstrated that this group was leaner compared with mice with a normal gut microbiota, even when the latter were fed with a lower caloric diet. When microbiota was transplanted from normal into germ-free mice, an increase in body fat of $60 \%$ was observed [3]. In other studies, also mainly performed in rodents, obesity has been linked with increased levels of Firmicutes [4]. However, others have not been able to confirm this [5-7].

Given the importance of the intimate association between colonic microbiota and host, the microbiota has been acknowledged as a metabolic organ [8]. This metabolic function highly depends on the type of substrates available to be fermented by the microbiota in the gut [9]. The major fermentation processes in the colon are saccharolytic and proteolytic fermentation. The main metabolites formed during saccharolytic fermentation are short-chain fatty acids (SCFA: namely: acetate, propionate and butyrate) with some intermediate metabolites including succinate, acrylate, lactate, formate, ethanol, and gases such as $\mathrm{H}_{2}, \mathrm{CH}_{4}$ and $\mathrm{CO}_{2}$ [10]. On the other hand, proteolytic fermentation leads to the production of acetate, propionate, butyrate and branched-chain fatty acids (BCFA) including principally iso-butyric, iso-valeric and 2-methylbutyric acids [11]. Furthermore, it also results in the production of phenols, indoles, ammonia, and amines, which are recognized as potentially toxic metabolites to the host [9].

Acetate, propionate and butyrate have been of particular interest in many studies. Ferchaud-Roucher et al. [12] observed that acetate may interfere directly with lipid metabolism and compelling evidence has shown propionate to lower fatty acid content in liver and plasma. Furthermore, it has been found that propionate reduces food intake, exerts immunosuppressive actions and probably improves tissue insulin sensitivity $[13,14]$. On the other hand, butyrate is metabolized by gut epithelial cells. It is responsible for $70 \%$ of their energy needs $[15,16]$ and acts as a signaling metabolite, affecting epithelial cell proliferation and differentiation $[17,18]$. Although L- and D- lactate are found to be present in low concentrations in stools from healthy subjects, it has been observed that humans have the ability to metabolize both isomers to pyruvate via the L-lactate dehydrogenase (L-LDH) and D-2-hydroxy acid dehydrogenase [19]. Furthermore, significant amounts of lactate 
could be converted to SCFA, especially to butyrate, by some bacteria [20,21]. For these reasons, its analysis was included in the present study.

As mentioned above, the concentration and ratio of SCFA produced in the gut depends, among others, on substrate availability and the composition of the intestinal microbiota [22]. Substrates for colonic fermentation include prebiotics. Prebiotics are defined as "non-digestible food ingredients that beneficially affect the host by selectively stimulating the growth and/or activity of one or a limited number of bacteria in the colon, thereby improving host health" [23]. Prebiotics also impact on the acidity of the lumen via the metabolic end-products (SCFA). By lowering the $\mathrm{pH}$ of the luminal content, prebiotics may exert a negative effect on harmful bacteria since acidification of the gut has been proposed as a mechanism that prevents the growth of pathogens [24]. The prebiotic property of influencing SCFA production in the gut may play an important role in the prevention and/or treatment of obesity.

With the increase of overweight and obesity worldwide and their devastating consequences on human health there is a growing interest to unravel the complex relationship between diet, gut microbiota, and host health. However, there is a lack of in vitro studies addressing such interaction experimenting with human gut microbiota. To our knowledge, one of the few studies that have investigated this role was performed by Sarbini et al. [25]. In this study, the fermentation of $\alpha$ gluco-oligosaccharides and inulin produced similar effects on bacterial population and metabolic activity in both lean and obese microbiotas. Therefore, besides including microbiota from lean and obese subjects, this study incorporated the analysis of different fermentable prebiotics: galacto-oligosaccharides (GOS), lactulose, apple fiber and sugar beet pectin.

The reasons why the tested substrates were chosen are: i) the potential of GOS and lactulose to beneficially influence gut microbiota composition has not been widely studied [26]. Rowland and Tanaka [27] reported that GOS increased lactobacilli and bifidobacteria and decreased enterobacteria in rats colonized with human fecal microbiota. This was supported by Bouhnik et al. [28] and Maathuis et al. [29] who found that GOS administered to adults [28] and to the TIM-2 system [29] led to a significant increase in (fecal) amounts of bifidobacteria. Nevertheless some studies have not been able to replicate the bifidogenic impact of GOS [24]. Furthermore, little is known about the metabolic products from the fermentation of GOS and most of the time it has been studied in combination with fructooligosaccharides (FOS). ii) Lactulose has been found to selectively stimulate and decrease the growth of certain bacterial groups [23], contribute to colonization resistance [24], increase the absorption of calcium [30] and is fermentable by the gut microbiota [24]. The use of lactulose has been mainly in the pharmaceutical 
Lean vs Obese: apple fiber, sugar beet, GOS, lactulose fermentation

industry, and it has been commercialized in the last years as a drink additive or food [24]. Therefore, the impact of its consumption on the host microbiota is of particular interest. iii) Dietary fibers like pectins are important for nutritional companies because of their cheap production and their beneficial effects on reducing glucose absorption, hypocholesterolemic effects and delayed gastric emptying [31,32]. Moreover, pectins may reduce the generation of ammonia [33] and the adhesion of pathogens to intestinal epithelial cells [34], in addition to their contribution in maintaining intestinal integrity [35]. Therefore, the influence of pectins on the intestinal microbiota composition was included here.

In the present work, the validated dynamic in vitro proximal colon model (TIM-2) developed by The Netherlands Organization for Applied Scientific Research (TNO) [36] was used to evaluate potential differences between the microbiotas originating from lean and obese individuals, and elucidate how energy is extracted from non-digestible carbohydrates or prebiotics in the form of SCFA products. The work contributes to the understanding whether dietary intervention helps to reduce obesity by suggesting that different fermentable carbohydrates are fermented differently by lean and obese microbiotas.

\section{Materials and Methods}

\section{Test compounds and control}

The compounds used as the carbohydrate source for the microbial fermentation in the in vitro system were GOS (97\%, degree of polymerization (DP) 2-6, average DP 3, FrieslandCampina, Beilen, The Netherlands), lactulose (98\%, SigmaAldrich, Zwijndrecht, The Netherlands), apple fiber (with a 60\% content of total sugars $45 \%$ of them from glucose and $23 \%$ of uronic acid residues; CSM, Bingen, Germany) and sugar beet pectin (average degree of methylation of 53, and a 58\% $\mathrm{w} \mathrm{w}^{-1}$ content of uronic acids; GENU® BETA pectin, CPKelco, Nijmegen, The Netherlands). A standard ileal efflux medium (SIEM; Tritium Microbiology, Veldhoven, The Netherlands) was used as a control and was modified from [37]. This medium contained the major non-digestible carbohydrates found in a normal western diet [29]. The preparation of starch (amylopectin and amylose) and nonstarch polysaccharides (NSP; pectin, xylan and arabinogalactan) present in SIEM, have been used by other authors to simulate the dietary carbohydrate portion in fermentation studies [29,38,39]. In absence of mucin, Gibson et al. [37] proposes this composite to provide a source of carbon during fermentation. As described by these authors, starch is included as a major polysaccharide due to evidence showing that this carbohydrate is highly abundant in the large intestine when compared with NSP. Furthermore, it is considered that resistant starch provides the 
largest proportion of energy to the colon [38]. Feed preparations were made containing approximately $7.5 \mathrm{~g}$ of fermentable carbohydrate per day, which constitutes $12 \% \mathrm{w} \mathrm{w}^{-1}$ of the total medium.

\section{Microbiota}

Homogenates of human feces were made from subgroups of healthy volunteers who were selected depending on their body mass index (BMI). The groups of participants were non-smokers and had not used antibiotics, prebiotics, probiotics or laxatives for at least 3 months prior to the donation.

Lean participants $(n=4)$ were recruited from a pool of volunteers at TNO Healthy Living (Zeist, The Netherlands). This group had an average BMI of $23.5 \pm 1.3 \mathrm{~kg} \mathrm{~m}^{-}$ 2 .

Obese participants $(n=4)$ were recruited from a pool of volunteers at Maastricht University Medical Center (The Netherlands). The group had an average BMI of $33.5 \pm 2.6 \mathrm{~kg} \mathrm{~m}^{-2}$.

Fresh fecal samples were directly collected by all the donors in a closed box with an anaerobic strip (AnaeroGen, Oxoid, Cambridge, UK) inside. The collection of boxes for the study was carried out in a time frame of $5 \mathrm{~h}$. As soon as a box was ready, it was stored in an anaerobic chamber (Bactron Anaerobic IV, Shel Lab, Cornelius, USA) to guarantee the best conditions for pooling. Donations from each category (lean or obese), were homogenized and mixed under strict anaerobic conditions to create a standardized microbiota stock. This allowed the performance of all experiments to start with the same microbial composition. Mixing was done with a Turrax (IKA Ultra turrax T25 digital, Staufen, Germany) with a physiological saline preparation/dialysate (content per litre: $2.5 \mathrm{~g} \mathrm{~K}_{2} \mathrm{HPO}_{4} \cdot 3 \mathrm{H}_{2} \mathrm{O}$, $4.5 \mathrm{~g} \mathrm{NaCl}, 0.005 \mathrm{~g} \mathrm{FeSO}_{4} \bullet 7 \mathrm{H}_{2} \mathrm{O}, 0.5 \mathrm{~g} \mathrm{MgSO}_{4} \bullet \mathrm{H}_{2} \mathrm{O}, 0.45 \mathrm{~g} \mathrm{CaCl}_{2} \bullet 2 \mathrm{H}_{2} \mathrm{O}, 0.05 \mathrm{~g}$ ox bile and $0.4 \mathrm{~g}$ cysteine hydrochloride), and glycerol $\left(14 \% \mathrm{w} \mathrm{w}^{-1}\right)$ as cryoprotective agent. Aliquots of the fecal homogenates $\left( \pm 11 \% \mathrm{w} \mathrm{w}^{-1}\right)$ were snap-frozen in liquid nitrogen $\left(-196^{\circ} \mathrm{C}\right)$ and were stored in a freezer at $-80^{\circ} \mathrm{C}$ [40]. Before being introduced into the system, the inoculum was thawed by $1 \mathrm{~h}$ immersion in a $37^{\circ} \mathrm{C}$ water bath.

Since this study demanded high throughput experiments, it was decided to use a pool of microbiota as an inoculum for the different experiments. We have previously shown that pooling does not affect microbiota composition [41].

\section{TIM-2 Experiments}

The TIM-2 system (Fig. 1) has been described in detail before [42]. In brief, the units were flushed with $\mathrm{N}_{2}$ (Fig. 1f) prior to the introduction of the inoculum, and throughout the remainder of the experiment. The system was maintained under this 
condition at $37^{\circ} \mathrm{C}$ using a temperature sensor (Fig. 1j; Easytem R31, Endress+Hauser, Nesselwang, Germany) for $96 \mathrm{~h}$ with the $\mathrm{pH}$ kept at or above 5.8 by automatic titration with $2 \mathrm{M} \mathrm{NaOH}$ (Fig. 1c). In order to remove water and fermentation products from the lumen a dialysate system (Fig. 1d; described in the following section) consisting of a semi-permeable hollow membrane ran through the lumen. For all the experiments, the speed of the dialysis fluid was set at $1.5 \mathrm{ml}$ $\min ^{-1}$.

After 24 and $48 \mathrm{~h}$ of fermentation $25 \mathrm{ml}$ of lumen sample was removed from the system to mimic the transit of chyme from the proximal to the distal colon [42].

The SIEM preparation mentioned above was gradually introduced into the system through a feeding syringe (Fig. 1i) in a total volume of $40 \mathrm{ml}$ in the adaptation period, while $180 \mathrm{ml}$ of a modified SIEM-preparation (in which the standard carbohydrate source in the medium was replaced by the test compound) was used over the $72 \mathrm{~h}$ of the test period at a rate of $2.5 \mathrm{ml} \mathrm{h}^{-1}$. Luminal content was maintained at a level of approximately $120 \mathrm{ml}$ in each unit by a level sensor (Fig. 1e; liquiphant FTL20-0025, Endress+Hauser, Maulburg, Germany).

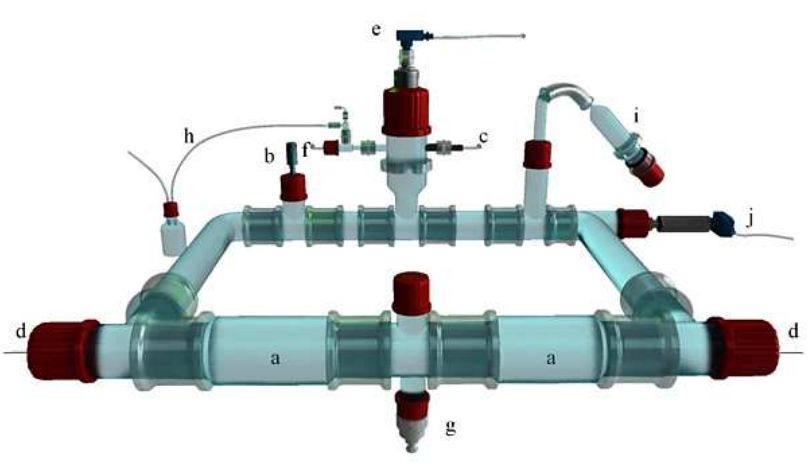

\section{Dialysate system}

The dialysate fluid used in the system contained per litre: in addition to the dial preparation described under "microbiota" section, $1 \mathrm{ml}$ of vitamin mixture containing per litre: $1 \mathrm{mg}$ menadione, $2 \mathrm{mg}$ biotin, $0.5 \mathrm{mg}$ vitamin $\mathrm{B}_{12}, 10 \mathrm{mg}$ pantothenic acid, 5mg nicotinamide, $5 \mathrm{mg} p$-aminobenzoic acid, and $4 \mathrm{mg}$ thiamin (all from Tritium Microbiology).

\section{Fermentation}

Each experimental unit of TIM-2 was filled to $120 \mathrm{ml}$ with approximately $70 \mathrm{ml}$ portion of fecal homogenate and $50 \mathrm{ml}$ of dialysate. After inoculation, the
Fig. 1. TIM-2 schematic, slightly modified from Maathuis et al. [42]. a) peristaltic compartments with a hollow membrane inside, b) $\mathrm{pH}$ sensor, c) $\mathrm{NaOH}$ secretion, d) dialysate system, e) level sensor, f) gaseous $\mathrm{N}_{2}$ inlet, g) sampling port, h) gas outlet, i) test compound + SIEM feeding syringe, $\mathrm{j}$ ) temperature sensor. 
microbiota was left to adapt (16h) to the new environment. After this adaptation period, the microbiota was deprived from any carbohydrate source (starvation) for $4 \mathrm{~h}$ in order to completely use the carbohydrates in SIEM [43], which provides the best condition to assess the effects of the desired test compound without bias of the SIEM component from the adaptation phase. Minekus et al. [36] explained that this starvation period was established when a lack of production of acids was observed in the system when the feeding line was turned off. This could be interpreted as the depletion of the fermentable carbohydrate.

After the starvation period, an initial lumen and dialysate portion were taken for later analysis $(\mathrm{t}=0)$ and the microbiota was immediately fed with preparations containing the substrates under study (Fig. 2). With samples from lumen and dialysate taken every $24 \mathrm{~h}$ of the experiment the production of SCFA, BCFA, lactate and ammonia was calculated. The concentration of metabolites at $\mathrm{t}=0$ was artificially set to zero. The microbial composition was measured at $\mathrm{t}=0$ and $\mathrm{t}=72$ in the luminal samples.

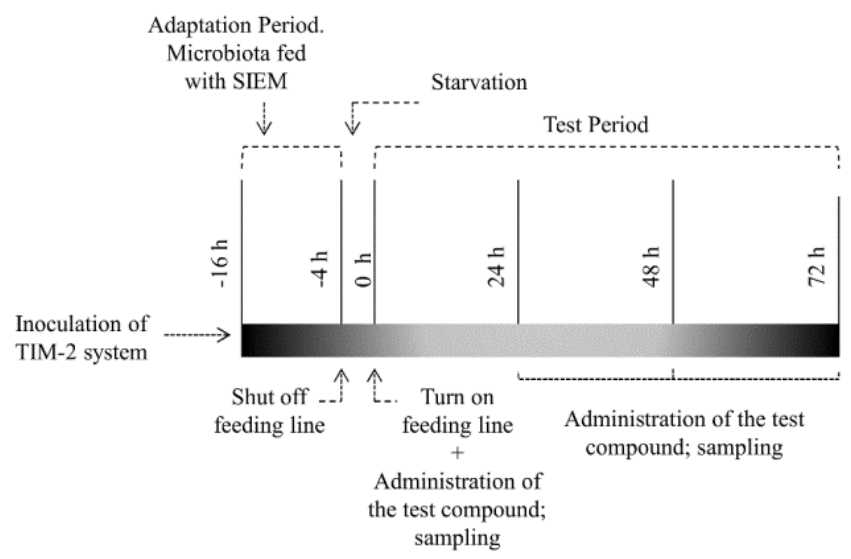

Fig.2. Study design.

\section{Analytical methods}

SCFA (acetate, propionate and butyrate), BCFA (iso-butyrate and iso-valerate), lactate and ammonia analyses were performed as described by Van Nuenen et al.[44]. Calibration lines were obtained by injecting known quantities of standard solutions, and amounts in the samples were calculated based on the calibration lines. Lactate and ammonia analyses were performed by Bio-aNAlytiX (Mook, The Netherlands). 
Lean vs Obese: apple fiber, sugar beet, GOS, lactulose fermentation

\section{Energy extraction}

Energy extraction in the form of SCFA was calculated using the following $\mathrm{kJ} \mathrm{mol}^{-1}$ values for acetate, propionate, butyrate and lactate respectively: 874, 1536, 2192 , and $1364[45,46]$.

\section{Molecular techniques}

DNA from the luminal samples was isolated using the AGOWA mag Mini kit (DNA Isolation Kit, AGOWA, Berlin, Germany), according to the manufacturer's instructions.

Generation of the PCR amplicon library was performed by amplification of V5-V7 hypervariable region of the small subunit ribosomal DNA gene (16S rDNA). Amplification was performed using the forward primer $785 \mathrm{~F}$ (5'GGATTAGATACCCBRGTAGTC-3') and reverse primer 1175R ('5ACGTCRTCCCCDCCTTCCTC-3). The primers were fitted with the Roche 454 (Branford, CT, USA) Adapter A (forward primer) and B (reverse primer), fused to the 5' end of the 16S rDNA bacterial primer sequences. The forward primer also included a unique nucleotide barcode. The amplification mix contained 2 units of Pfu Ultra II Fusion HS DNA polymerase (Stratagene, La Jolla, CA, USA) and 1x Pfu Ultra II reaction buffer (Stratagene), 200 $\mu$ M dNTP PurePeak DNA polymerase Mix (Pierce Nucleic Acid Technologies, Milwaukee, WI, USA), and $0.2 \mu \mathrm{M}$ of each primer.

After an initial denaturation $\left(94^{\circ} \mathrm{C} ; 2 \mathrm{~min}\right), 30$ cycles were performed that consisted of denaturation $\left(94^{\circ} \mathrm{C} ; 30 \mathrm{sec}\right)$, annealing $\left(50^{\circ} \mathrm{C} ; 40 \mathrm{sec}\right)$, and extension $\left(72^{\circ} \mathrm{C} ; 80\right.$ $\mathrm{sec})$. Samples with DNA recovery of equal or less than $10 \mathrm{pg}^{\mathrm{l}^{-1}}$ of DNA were cycled 35 times using the same protocol.

Amplicons were size checked and quantified by gel electrophoresis and Quant-iT Picogreen dsDNA Assay (Invitrogen, Carlsbad, CA, USA) on the Tecan Infinite M200 (Tecan Group Ltd, Männedorf, Switzerland). Amplicons of the individual samples were equimolar pooled and purified from agarose gel by means of QIAquick Gel Extraction Kit Protocol (Qiagen, Hilden, Germany).

The library was sequenced unidirectionally in the forward direction (A-adaptor) in one run in the 454 GS-FLX-Titanium Sequencer (Roche) by Keygene (Wageningen, The Netherlands).

\section{Sequence processing and analyses}

FASTA-formatted sequences and corresponding quality scores were extracted from the .sff data file generated by the GS-FLX-Titatium sequencer using the GS Amplicon software package (Roche). 
All data extraction, pre-processing, analysis of operational taxonomic units (OTUs), and classifications were performed using modules implemented in the Mothur v. 1.20.0. software platform [47] as in Roeselers et al. [48].

In brief, unique barcodes were used to sort sequences by sample of origin. Subsequently, barcodes, primer sequences and low quality data (containing ambiguous base calls $(\mathrm{N})$ in the sequence, more than 8 homopolymers anywhere in the sequence, shorter than $50 \mathrm{nt}$ after trimming with a window average below 35 , or a length $>500$ or $<200 \mathrm{bp}$ ) were removed. The data set was simplified by using the "unique.seqs" command to generate a non-redundant (unique) set of sequences. Sequences were 'denoised' using the "pre.cluster" command [49]. Unique sequences were aligned using the "align.seqs" command and an adaptation of the Bacterial SILVA SEED database as a template [50]. In order to ensure that comparable regions of the $16 \mathrm{~S}$ rDNA gene were analyzed across all reads, sequences that started before the 2.5-percentile or ended after the 97.5-percentile in the alignment were filtered.

A total of 149220 potentially chimeric sequences were detected and removed using the "chimera.uchime" command [51]. High quality aligned sequences were classified by using the RDP-II naïve Bayesian Classifier [52] using a 60\% confidence threshold. Aligned sequences were also clustered into operational taxonomic units (OTUs; defined by $97 \%$ similarity) and were calculated by the average linkage clustering method. Unclassified sequences were also grouped in OTUs and were represented with a random number to distinguish them from other unclassified OTUs found within the same phyla. Community profiles were compared using the weighted Unifrac metric [53].

\section{Data presentation}

The experiments were performed in duplicate $(n=2)$ per substrate, except for control $(n=3)$. To avoid unnecessary repetition, this is not indicated further in the text or graphs in the results section. Results are displayed as average of these duplicates/triplicates. Since the sample size is small no statistical analysis was performed. For simplicity of reading, substrates in the following sections are tagged with the letter L or O (e.g. substrate-L, substrate-O) in order to refer to the fermentation experiments using the inoculum from lean $(-\mathrm{L})$ or obese $(-\mathrm{O})$ subjects.

\section{Ethical approval}

Studies using fecal donations from healthy volunteers do not require medical ethical committee approval in The Netherlands since they are considered as noninvasive. Nevertheless, all participants provided informed consent. 


\section{Results}

\section{Metabolites}

The cumulative amount of SCFA including lactate produced in TIM-2 (Fig. 3) was lower after $72 \mathrm{~h}$ in the fermentation experiments with GOS-L $(123 \mathrm{mmol})$ compared to GOS-O (162 mmol). Also lactulose-L resulted in lower production than lactulose-O with values of $155 \mathrm{mmol}$ and $173 \mathrm{mmol}$, respectively. In contrast to the results obtained for GOS and lactulose, the fermentation of apple fiber and sugar beet pectin with microbiota from lean subjects exhibited a higher cumulative production of SCFA compared with microbiota from obese subjects. With respect to apple fiber-L, the amounts were higher $(108 \mathrm{mmol})$ than with apple fiber-O (92 $\mathrm{mmol})$. Sugar beet-O $(103 \mathrm{mmol})$ was lower than sugar beet-L $(130 \mathrm{mmol})$. The amount for the SIEM control-L $(125 \mathrm{mmol})$ and that for control-O $(124 \mathrm{mmol})$ were very similar. The averaged molar ratios (\% of total SCFA) are shown in Fig. S1. In general, acetate was the main product in the different fermentations followed by $n$-butyrate (with the exception of lactulose-O and sugar beet-L in which it was exceeded by lactate and propionate, respectively) and propionate. Specifically in the case of lactate, it can be observed that its production was higher in GOS-O and lactulose-O experiments when compared to the other substrates (Fig. 3 and Fig. S1).
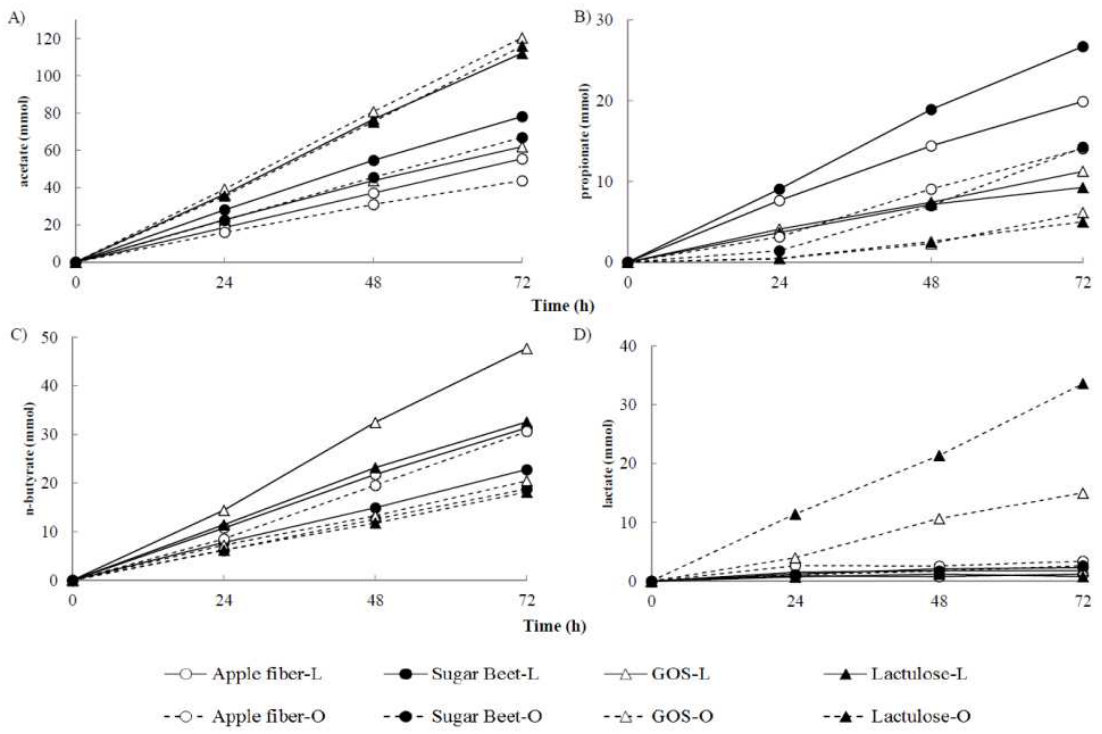

Fig. 3. Cumulative production of SCFA. A) acetate, B) propionate, C) $n$-butyrate and D) lactate produced during $72 \mathrm{~h}$ fermentation of 
apple fiber, sugar beet pectin, GOS and lactulose using microbiota from lean or obese subjects.

Fermentations of the different substrates using microbiota from lean subjects in TIM-2 resulted in higher amounts of BCFA and ammonia compared to fermentations using the obese inocula (Table 1). An approximately 4.5 and $\sim 10.5$ fold increase of BCFA were observed for GOS-L and lactulose-L compared to the O-microbiota, whilst both presented 1.1 fold increase in ammonia. Interestingly, for GOS and lactulose, the amounts of BCFA and ammonia were lower than their respective controls for both $\mathrm{L}$ and $\mathrm{O}$ inocula. The cumulative BCFA produced by the lean microbiota in the incubations with apple fiber and sugar beet pectin were also higher: $\sim 1.7$ fold for apple fiber and $\sim 2.9$ fold for sugar beet pectin. The ammonia increase in both substrates was $\sim 1.1$ and $\sim 1.3$ fold, respectively.

Table 1. Cumulative production of BCFA and ammonia after $72 \mathrm{~h}$ of addition of apple fiber, sugar beet pectin, GOS or lactulose.

\begin{tabular}{|lcc|}
\hline \multicolumn{3}{|c|}{ Total amount } \\
\hline Substrate & $\begin{array}{c}\text { i } \\
\text { i-butyrate }+ \\
\text { i-valerate }\end{array}$ & Ammonia \\
\hline Apple fiber-L & 4.5 & 65 \\
Sugar beet-L & 4.1 & 74 \\
GOS-L & 1.8 & 44 \\
Lactulose-L & 2.1 & 36 \\
Apple fiber-O & 2.6 & 59 \\
Sugar beet-O & 1.4 & 59 \\
GOS-O & 0.4 & 39 \\
Lactulose-O & 0.2 & 33 \\
Control-L & 3.9 & 74 \\
Control-O & 0.7 & 52 \\
\hline
\end{tabular}

\section{Energy extraction}

Three day fermentation of GOS provided an energy value in the form of SCFA and lactate of $180 \mathrm{~kJ}$ in experiments with both $\mathrm{L}$ and $\mathrm{O}$ microbiota, despite their total SCFA concentrations (and ratios) being different. The amount of energy extracted is higher as their respective controls (176 and $162 \mathrm{~kJ}$, respectively for control-L and $-\mathrm{O})$. Fermentation of lactulose-L extracted $185 \mathrm{~kJ}$ in the form of SCFA and lactate, whereas for lactulose-O this was 195kJ (Fig. 4). These results suggest that GOS and lactulose had a greater energy value per gram carbohydrate when compared to control. 


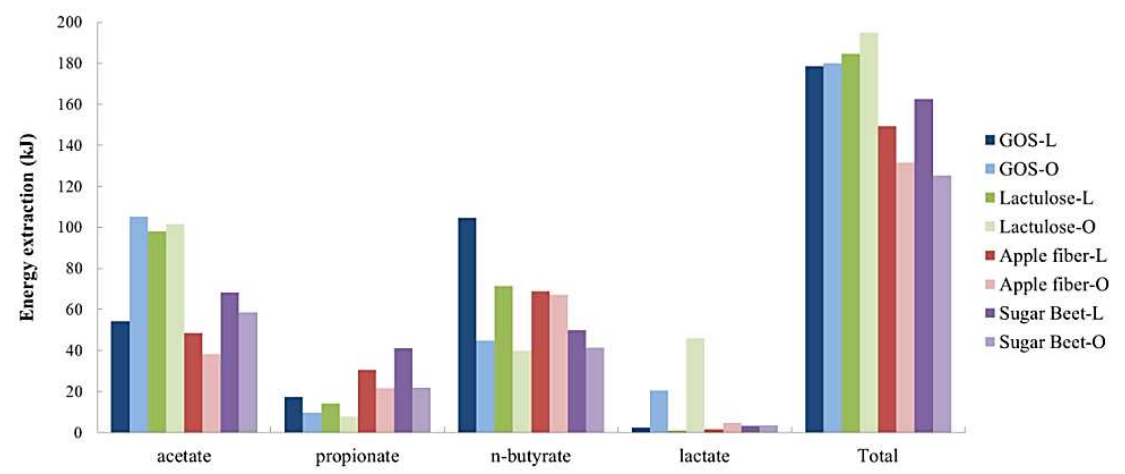

Fig. 4. Energy extraction. Values obtained after $72 \mathrm{~h}$ fermentation of GOS, lactulose, apple fiber and sugar beet using microbiota from lean or obese subjects. Total energy extraction for control-L and -O were 176 and $162 \mathrm{~kJ}$, respectively. The calculation was performed by multiplying the SCFA and lactate produced by their corresponding $\mathrm{kJ}$ $\mathrm{mol}^{-1}$ value as explained in the materials and methods section.

Apple fiber had an energy value of 149 and $132 \mathrm{~kJ}$ after $72 \mathrm{~h}$ of fermentation, for experiments with lean and obese inocula, respectively. That is less compared to their respective controls. Fermentation of sugar beet-L extracted $162 \mathrm{~kJ}$ of energy whereas for sugar beet-O this was $125 \mathrm{~kJ}$. These results suggest that apple fiber and sugar beet pectin in both types of fermentations had a lower energy value per gram when compared to control, and that contrary to expectations, the lean microbiota extracted more energy from these two substrates than the obese microbiota.

\section{Phylogenetic analysis of the microbiota at $t=0$}

After the adaptation period in the TIM-2 system, Firmicutes were more predominant in the microbiota prepared with the homogenates of obese subjects compared to that prepared with the homogenates from lean subjects (Fig. 5). The average abundance at time zero was $92 \pm 3.3 \%(\mathrm{n}=10)$ and $74 \pm 1.0 \% \quad(\mathrm{n}=11)$, respectively. On the other hand, Bacteroidetes were more dominant in the microbiota prepared with homogenates from lean subjects with an average abundance of $22 \pm 0.9 \%(n=11)$ compared with the microbiota prepared with homogenates from obese subjects $(3.6 \pm 1.9 \% ; n=10)$. 


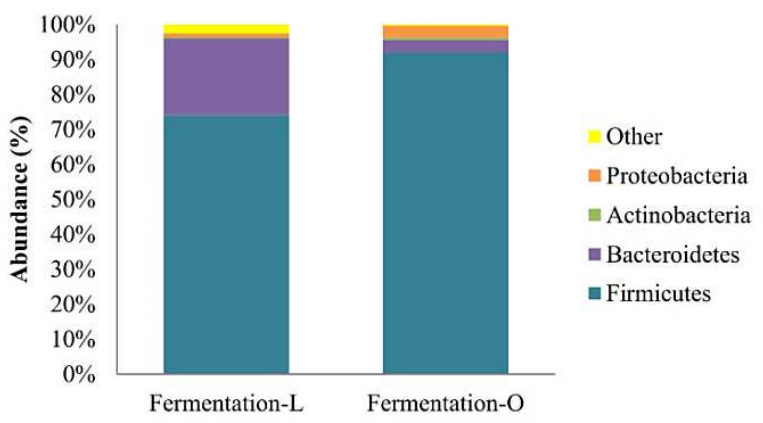

Fig. 5. Abundance (\%) of Firmicutes, Bacteroidetes, Actinobacteria, Proteobacteria and others. Phyla were analyzed in the fermentations with microbiota from lean $(\mathrm{L})$ or obese $(\mathrm{O})$ subjects at time zero. Data from all runs (fermentation- $\mathrm{L} n=11$; fermentation- $\mathrm{O} n=10$ ) were taken. The category presented as "Other" includes the sum of Verrucomicrobia, Lentisphaerae, Tenericutes and unclassified bacteria which constituted a low abundance in the samples.

A low proportion of Actinobacteria and Proteobacteria was observed in the microbiota from the obese $(0.7 \pm 0.3 \%$ and $3.3 \pm 1.1 \%)$ and lean individuals $(0.5 \pm 0.2 \%$ and $1.0 \pm 0.3 \%)$.

\section{Changes observed in the microbiota after the experiments in TIM-2}

A phyla and genus level analysis was performed using the sequencing data in which changes in bacterial abundance were measured by considering the changes in the two controls ( $-\mathrm{L}$ and $-\mathrm{O}$ ) as the baseline. The ratio between the shift in the composition of the microbiota in the experiments using different substrates and the shift of the microbiota fed with the control carbohydrate was calculated, presented as a "fold increase or decrease". Several groups of bacteria showed changes in their relative abundance (Table 2), as detailed below.

Table 2. Relative change of bacterial genera after $72 \mathrm{~h}$ of fermentation experiments in TIM- $2^{1}$. The ratio between a sampling time point and $t_{-16}$ was calculated (e.g., $t_{72} / t_{-16}$ ). The ratio for this value and the control was then determined to obtain fold changes. A value equal to 1 indicates no change; a value of $>1$ indicates an increase; and a value of $<1$ indicates a decrease of the respective microbial genera. Sequences of the $16 \mathrm{~S}$ rDNA gene that are not fully available in the database but represent a high similarity in the genus tagged are presented as unclassified groups and are listed in the table as OTU\#\#, with \#\#\# being a number. 


\begin{tabular}{|c|c|c|c|c|c|c|c|c|c|}
\hline Phyla & Genus & $\begin{array}{l}\text { Apple } \\
\text { fiber-L }\end{array}$ & $\begin{array}{r}\text { Sugar } \\
\text { beet-L }\end{array}$ & $\begin{array}{c}\text { GOS- } \\
\text { L }\end{array}$ & $\begin{array}{c}\text { Lactulose- } \\
\text { L }\end{array}$ & $\begin{array}{c}\text { Apple } \\
\text { fiber-O }\end{array}$ & $\begin{array}{c}\text { Sugar } \\
\text { Beet-0 }\end{array}$ & $\begin{array}{c}\text { GOS- } \\
\mathbf{0}\end{array}$ & $\begin{array}{c}\text { Lactulose- } \\
\text { o }\end{array}$ \\
\hline \multirow{32}{*}{ Firmicutes } & Catenibacterium & 215.0 & & 0.5 & 15.0 & & & & \\
\hline & Anaerostipes & 46.4 & & 0.6 & 22.7 & 27.2 & & 6.2 & 29.7 \\
\hline & Enterococcus & 28.7 & & & & 2.1 & 0.4 & 5.0 & 7.6 \\
\hline & Clostridium $I V$ & 19.3 & 1.4 & 0.1 & 0.2 & & & & \\
\hline & Clostridium XlVb & 17.80 & 20.6 & 0.03 & 11.9 & & & & \\
\hline & OTU 5111 & 6.1 & 1.1 & 0.04 & & & & & \\
\hline & Blautia & 5.7 & 1.5 & 2.0 & 4.8 & 2.7 & 0.4 & 29.7 & 50.8 \\
\hline & Acetivibrio & 5.2 & 3.6 & & & & & & \\
\hline & Coprococcus & 3.0 & 1.1 & 0.1 & 1.8 & 16.0 & 2.3 & 1.1 & 6.6 \\
\hline & Roseburia & 1.8 & 0.1 & & 1.5 & & 0.2 & & \\
\hline & Erysipelotrichaceae incertae sedis & 1.60 & 0.4 & 0.0 & 16.8 & & & & \\
\hline & OTU 2128 & 1.6 & 0.3 & 0.1 & 0.3 & 1.6 & 0.6 & 3.8 & 0.6 \\
\hline & OTU 1291 & 1.5 & 1.0 & 0.2 & 0.5 & 6.4 & 6.9 & 27.1 & 2.0 \\
\hline & Clostridium XlVa & 1.4 & 0.4 & 4.9 & 0.3 & & & & \\
\hline & Faecalibacterium & 1.2 & 6.0 & 0.5 & 0.6 & 1.4 & 8.1 & 22.2 & 13.5 \\
\hline & Dorea & 1.1 & 0.2 & 0.1 & 0.7 & 46.1 & 6.2 & 38.5 & 48.2 \\
\hline & OTU 2620 & 0.84 & 0.8 & 0.01 & 0.1 & & 9.9 & 6.4 & 8.8 \\
\hline & Ruminococcus & 0.56 & & & & & & & \\
\hline & Subdoligramulum & 0.47 & 0.6 & 0.01 & 0.1 & 0.03 & 0.8 & & 0.4 \\
\hline & OTU 2832 & 0.31 & & 0.04 & 0.1 & & & & \\
\hline & Oscillibacter & 0.3 & 0.7 & 0.02 & 0.3 & & & & \\
\hline & Lachospiracea incertae sedis & 0.10 & 0.1 & 1.4 & 0.2 & 22.96 & 4.0 & 0.4 & 0.3 \\
\hline & Dialister & 0.04 & 0.4 & 0.04 & 0.1 & & & & \\
\hline & Lactobacillus & & & & & 8.5 & 0.2 & 0.9 & 4.9 \\
\hline & Lactococcus & & & & & & 0.2 & 13.4 & \\
\hline & Streptococcus & & & 33.1 & & 4.8 & 0.2 & 2.7 & 7.6 \\
\hline & Clostridium sensu stricto & & & & & 1.2 & 0.3 & 1.1 & 1.4 \\
\hline & Anaerococcus & & & & & & & & 0.8 \\
\hline & Anaerororax & & 0.6 & & 0.2 & & & & \\
\hline & Clostridium XI & & & & & & & & 5.7 \\
\hline & Butyricicoccus & & & & & 0.1 & 0.2 & 1.0 & 0.2 \\
\hline & Allisonella & & & & & 3.6 & 4.6 & 2.8 & 9.3 \\
\hline \multirow{8}{*}{ Bacteroidetes } & Parabacteroides & 6.5 & 2.8 & 2.1 & 2.0 & 0.3 & 6.1 & 0.4 & 0.2 \\
\hline & Bacteroides & 4.3 & 21.6 & 9.1 & 1.2 & 1.9 & 2.5 & 0.2 & 0.1 \\
\hline & OTU 2171 & 3.81 & 0.6 & 7.2 & 0.3 & & & & \\
\hline & OTU 2145 & 1.8 & 1.3 & 0.3 & 0.6 & & & & \\
\hline & Alistipes & 1.45 & 0.6 & 0.05 & 3.8 & & & & \\
\hline & Xylanibacter & 1.1 & 0.9 & 0.1 & & & & & \\
\hline & Prevotella & 0.6 & 0.8 & 0.01 & 0.01 & & & & \\
\hline & Barnesiella & 0.1 & & & 0.2 & & & & \\
\hline \multirow[t]{3}{*}{ Actinobacteria } & Bifidobacteritum & & 12.5 & 600.1 & 4338.1 & 0.04 & 0.3 & 99.5 & 890.1 \\
\hline & Collinsella & & & 0.2 & 0.3 & & & & \\
\hline & OTU 4114 & & & & & & 2.0 & 2.8 & 5.5 \\
\hline \multirow[t]{4}{*}{ Proteobacteria } & Parasutterella & 10.7 & 9.9 & 0.5 & 27.6 & & & & \\
\hline & Gemmiger & 0.9 & 1.4 & & 0.2 & & & & \\
\hline & Sutterella & & & 0.2 & & & & & \\
\hline & OTU 1144 & & & & & 1.1 & 0.32 & 21 & 8.9 \\
\hline Unclassified & OTU 1111 & 0.5 & 0.8 & 0.0 & 0.1 & 0.04 & 0.02 & 0.01 & 0.01 \\
\hline
\end{tabular}

GOS and lactulose

The dynamics for Firmicutes and Bacteroidetes was different in all the fermentations with GOS and lactulose. GOS-L presented an increase in Firmicutes with the biggest raise in Streptococcus with a fold increase of 33, whereas GOS-O exhibited the biggest increase in Dorea spp. (39 fold). The fermentation with lactulose-L stimulated the growth of Anaerostipes spp. (23 fold) whilst lactulose-O exhibited a remarkable increase of Blautia spp. (51 fold).

A modest increase in Bacteroidetes was observed in the experiments with the inoculum prepared from lean subjects. GOS-L fermentation led to an increase in Bacteroides spp. ( 9 fold) whereas lactulose-L exhibited a moderate increase in Alistipes spp. (4 fold). Both GOS-L and lactulose-L presented a 2 fold increase in 
Parabacteroides spp. In the case of the experiments with the inoculum from obese subjects a general decrease of Bacteroidetes with respect to control was observed for both GOS and lactulose. The bacterial group corresponding to Parabacteroides spp. presented the lowest values: 0.38 fold (or 2.6 fold decrease) and 0.21 fold (4.8 fold decrease) for GOS and lactulose, respectively.

An outstanding growth in the group of Actinobacteria was found after 3 days of experiments with both GOS and lactulose as shown in Table 2. Lactulose-L presented a robust increase (4300 fold) compared with control followed by lactulose-O (890 fold), GOS-L (600 fold) and GOS-O (100 fold).

In the case of Proteobacteria there was a substantial increase in Parasutterella spp. in lactulose-L (28 fold). Although reported as an unclassified OTU (1144) by the sequencing analysis, a large increase was also observed for GOS-O (21 fold) and lactulose-O (9 fold) in this genus. A decrease of 0.52 fold of Proteobacteria was found in GOS-L in the specific case of Parasutterella spp.

Apple fiber and sugar beet pectin

OTUs from Firmicutes which were observed to increase the most for each fermentation that was carried out with apple fiber and sugar beet pectin corresponded to Catenibacterium spp. (215 fold; apple fiber-L), Dorea spp. (46 fold; apple fiber-O), Clostridium XIVb (21 fold; sugar beet pectin-L) and Faecalibacterium ( 8 fold; sugar beet pectin-O).

For Bacteroidetes, the fermentations with apple fiber-L and sugar beet pectin-O presented the same increase of Parabacteroides spp. (each 6 fold). Bacteroides spp. was found to increase ( 22 fold) in sugar beet pectin-L.

The proliferation of microorganisms from the group of Actinobacteria was not stimulated in both fermentations (-L and $-\mathrm{O}$ ) with apple fiber. On the other hand, experiments with sugar beet pectin-L were found to stimulate the growth of Bifidobacterium (12 fold) whilst a moderate increase ( 2 fold) of unclassified Actinobacteria (OTU 4114) was observed in sugar beet pectin-O.

On the other hand, increases in some Proteobacteria groups were observed in experiments with the lean microbiota such as the case apple fiber-L and sugar beet pectin-L which showed a similar increase in Parasutterella spp. (11 and 10 fold, respectively).

\section{Discussion}

The differences between human microbiotas from lean and obese individuals in terms of composition and activity, and their contribution to obesity through energy extraction in the form of SCFA and lactate was studied in vitro by fermenting 
Lean vs Obese: apple fiber, sugar beet, GOS, lactulose fermentation

several non-digestible, but fermentable carbohydrates. The golden standard to test this would be in a clinical trial. However, such trials are highly expensive and present certain limitations in design due to throughput and ethical constrains. Therefore, before going to sometimes rather invasive human intervention studies, in vitro models closely mimicking the microbial metabolism in the human intestine can be used to get further insight in the complex fermentation processes mediated by the gut microbiota.

TIM-2 is such an in vitro model that closely mimics the fermentation by the microbiota in the human large intestine by allowing the growth of a highly complex, stable and dense $\left(\sim 10^{11}\right.$ colony forming units $\left./ \mathrm{ml}\right)$ active microbiota $[43,54]$. The variations in the system are small, due to the fact that the experiments are computer-controlled and a standardized microbiota is used for each set of experiments which is derived from host subjects of interest [6]. In addition, the system has been validated analyzing i) the composition, ii) the enzymatic activity and iii) the production and concentration of SCFA of the microbiota used for the experiments [36], based on data from sudden-death individuals. Overall, the model provides the opportunity to study the human microbial ecosystem and the metabolism of test substrates under standardized conditions [40,42]. However, a limitation of the study set up may be the fact that a pool of microbiota rather than the microbiota from a single individual was used in the experiments. This is criticized mainly due to lack of stability of the mixed inoculum due to bacterial interactions. Nevertheless, this was done with the aim of working with i) a standardized microbiota that can be used for multiple (up to 100) different experiments, and ii) a more diverse population of bacteria and thus the use of a more representative microbiota for the whole population.

To our knowledge no comparative experiments have been performed using a single or mixed fecal inoculum. However, in a recent study performed by us in TIM-2, it was possible to demonstrate that the use of a pool of microbiota for in vitro studies does not result in a bacterial community with an aberrant profile and activity compared to that normally obtained from single donors [41]. In other studies using TIM-2 (pooled microbiota) as well as by the Ghent University in their Simulator of the Human Microbial Ecosystem (SHIME) (single fecal inoculum) with arabinoxylan very similar metabolic activity of the microbiota was observed [55]. In addition, previous data using the individual inocula from 10 donors has shown that the microbial activity of the individual donors was extremely similar, despite differences in microbiota composition [56], corroborating the hypothesis that there is substantial functional overlap in the microbiota of different individuals and that a standardized mixture of gut microbiota can be used for these types of experiments. 


\section{Metabolites produced}

Based on experiments with ${ }^{13} \mathrm{C}$-labeled substrates, it was shown that SCFA make up $>95 \%$ of the metabolites produced by the microbiota when fermenting a carbohydrate substrate [43]. Therefore, we focused on these metabolites when determining energy extraction. The fermentation of complex carbohydrates by bacteria has been considered as the major quantitative source of SCFA in the colon. Nevertheless, it is important to consider that breakdown of peptides and proteins do also contribute to the formation of SCFA as seen in vivo and in vitro [57]. Therefore, the possibility exists that some of the observed SCFA produced in the different experiments could also come from the fermentation of proteins. However, only the carbohydrate part of the medium was changed between the different variables tested. Consequently, the levels of protein in the diet used in the experiment and their conversion to SCFA are expected to be fairly similar for every experiment as well (including controls). Hence, the contribution of proteins to the energy extracted by the microbiota in terms of SCFA is also believed to be fairly similar regardless of the type of the test compound studied.

In the current experiments, low levels of lactate were found for both GOS-L and lactulose-L. On the other hand, high proportions of butyrate found in both fermentations were observed (Supplemental Online Material; Fig. S1) likely as a result of conversion of lactate into butyrate [58]. Butyrate is considered a healthy microbial metabolite [59]. From this, it is proposed that GOS and lactulose in lean people could boost a healthier colonic environment through conversion of lactate into butyrate.

In fermentations with GOS-O and lactulose-O there is a slight increase in total SCFA produced from both test compounds (1.3 fold for GOS-O and 1.1 fold for lactulose-O) compared to lean. It is important to highlight the fact that an increase in lactate was observed in these experiments as well which can be interpreted that GOS and lactulose are fermented faster in obese than in lean subjects, since lactate normally only accumulates when fermentation is fast [44].

Apple fiber and sugar beet pectin were more poorly fermented in all the experiments when compared with control, GOS and lactulose, as observed by the total production of SCFA and energy extraction. It can be observed that these substrates were not equally used by the microbiota from lean and obese subjects as evidenced by the nature of SCFA produced from the fermentation of these compounds. In contrast to the results obtained for GOS and lactulose, the fermentation of pectins with microbiota from lean subjects exhibited a higher cumulative production of SCFA compared with microbiota from obese subjects (1.2 fold for apple fiber-L and 1.3 fold for sugar beet pectin-L), suggesting a 
potential relative reduction of the presence of pectate lyases and other enzymes involved in pectin fermentation in the obese microbiota.

It is proposed that the sugar composition from the different test compounds could influence the type of the SCFA produced. Salvador et al. [60] found a correlation between high concentrations of acetate and uronic acid content from sugar beet in fermentations with fresh feces from healthy volunteers in an in vitro batch system. The results from the present study are in agreement with Salvador et al. Fermentations with sugar beet pectin in our experiments exhibited a higher amount of acetate when compared to apple fiber ( 1.4 fold in fermentation-L and 1.5 fold in fermentation-O) (Fig. 3). The sugar beet pectin contained $80 \mathrm{~mol} \%$ of uronic acid whereas apple fiber only had $23 \mathrm{~mol} \%$. Furthermore, Salvador et al. also demonstrated that butyrate production from fiber fermentation could be positively associated with the content of xylose. Taking into account that apple fiber contained $6 \mathrm{~mol} \%$ of xylose whilst sugar beet lacked xylose, the present study is in accordance with the findings from these authors, as we show here that apple fiber exhibited a higher butyrate production when compared to sugar beet pectin (1.3 fold in fermentation- $\mathrm{L}$ and 1.6 in fermentation-O).

In the experiments with microbiota-O, the addition of GOS and lactulose suppressed the formation of putrefactive metabolites compared to control. The results obtained demonstrate the potential of GOS and lactulose to reduce toxic metabolic products by repressing protein fermentation in obese subjects, providing good indication of their prebiotic effect.

\section{Energy extraction}

A higher production of SCFA by the microbiota will consequently lead to a higher energy input to the host, as $95 \%$ of the SCFA produced by the microbiota are absorbed, and only 5\% is excreted in feces [61]. Yet, it is important to realize that each SCFA contributes differently to the energy extracted from fermentable carbohydrates. Butyrate contains more energy than acetate and propionate (2192, 874 and $1536 \mathrm{~kJ} \mathrm{~mol}^{-1}$, respectively) [6]. In this study, the energy extracted from GOS and lactulose for both types of fermentation was similar. However, it was observed that $n$-butyrate was higher in the fermentations with the lean inocula and therefore, the caloric input through $n$-butyrate was increased. Both apple fiber and sugar beet pectin exhibited more energy input in experiments with the lean inocula. The obese microbiota fermenting the fiber and the pectin produced less energy from acetate and propionate compared with the lean one. The energy input from propionate was higher in sugar beet-L when compared to the other fermentations (Fig. 4). It could be that, as obese subjects have a different type of diet (low in complex carbohydrates), this results in a non-adapted microbiota, not fully capable 
of degrading pectins. Important to consider as well is the $\mathrm{pH}$ of the system ( $\mathrm{pH} 5.8$, simulating the proximal colon) which might also have inhibited the activity of pectate lyases. Further experiments are carried out in order to confirm these hypotheses.

It is believed that the additional energy input to the host from its microbiota could be used for glucose and de novo hepatic triglyceride synthesis [62]. A slight increase of just $1 \%$ in the microbial metabolic activity may increase the input of 20 kcal day ${ }^{-1}$ to the host (based on a diet of $2000 \mathrm{kcal} \mathrm{day}^{-1}$ ) which could lead to a weight gain of approximately $1 \mathrm{~kg}$ per year [62]. For this reason, the impact of the activity of gut bacteria on obesity in humans should not be underestimated.

\section{Phylogenetic analysis of the microbiota at $\mathrm{t}=\mathbf{0}$}

The phylogenetic analysis of the inoculum prepared with microbiota- $\mathrm{L}$ and microbiota-O at time zero (after the adaptation period) demonstrates that both individual groups cluster closely together depending on the source of the inoculum (Supplemental Online Material; Fig. S2). This demonstrates the difference between these two types of inocula at the starting point of the experiments. Furthermore, this shows the high reproducibility of the in vitro model.

When calculated, the ratio of Firmicutes/Bacteroidetes in the starting microbiota prepared with donations from obese subjects at time zero was found to be higher $(87 \pm 168)$ compared to the starting microbiota prepared with donations from lean subjects (4.5 \pm 4.2$)$. In studies, mainly performed in rodents, obesity has been linked with reduced levels of Bacteroidetes [5-7]. In fact, in a study conducted in obese mice resulting in more efficient energy harvesting compared to lean counterparts, this was associated with a higher ratio of Firmicutes/Bacteroidetes in these animals [4], which corroborates our observations. Although it is hypothesized that this increased ratio might either promote fat storage or lead to a different energy uptake/storage in the body, the exact mechanisms are not known. In contrast, other studies such as the one conducted by Duncan et al. [5] did not find any evidence about the function of proportions of Bacteroidetes and Firmicutes in human obesity.

\section{Changes observed in the microbiota after the experiments in TIM-2}

Bifidobacterium from both lean and obese subjects were clearly stimulated by GOS and lactulose during the 3 day fermentation period, as the increase in this group of microorganisms was much higher compared with the other genera. The metabolic activity of bifidobacteria has been found to be beneficial to the host, e.g., it has shown to inhibit the colonisation of pathogenic microorganisms, and have anticarcinogenic effects [63]. Important to realize is that not all strains of 
Lean vs Obese: apple fiber, sugar beet, GOS, lactulose fermentation

bifidobacteria have probiotic effects. It has been found that some bacteria from this genus are implicated in mucin degradation, whilst others are recognized as $\beta$ glucosidase producers hypothesized to be involved in colon cancer promotion [64]. A robust increase of Bifidobacterium spp. likely causes a higher production of acetate and lactate [29], which is indeed evidenced by the higher ratios of these SCFA in those experiments where bifidobacteria are increased.

The markedly increased abundance of Catenibacterium spp in apple fiber-L could explain why the cumulative SCFA ratios were higher compared with sugar beet-L. According to Kageyama and Yoshimi [65], the major end products from the fermentation of carbohydrates by Catenibacterium spp. are acetate, butyrate and lactate. Thus, this demonstrates the high yield in the energy extraction from the fermented apple fiber in lean subjects.

The results also evidence the potential benefit of apple fiber and sugar beet pectin for obese and lean individuals, respectively. On the one hand, it is presumed that Dorea spp. (46 fold increase in apple fiber-O) are related to blunting inflammatory processes [66,67]. On the other hand, Clostridium cluster XIV spp. (21 fold increase in sugar beet-L) may lead to a reduced circulation of inflammatory markers and body weight [68].

The prebiotic effect of sugar beet pectin is also demonstrated by the stimulation of the growth of Faecalibacterium spp. F. prausnitzii is considered as an antiinflammatory species which stimulates the production of butyrate [69]. As Furet et al. [70] found low abundance of this bacteria in obese subjects, the potential beneficial effect of sugar beet pectin for these subjects is highlighted by the observed increase in Faecalibacterium. More studies at the level of bacterial species and the measurement of other markers including especially inflammatory ones are recommended in order to assess the effect of these two substrates in the colonic homeostasis in humans.

The results from the present study suggest differences in the extraction of energy in the form of SCFA as well as dynamic changes in the composition of the gut bacteria which may be strongly associated with the substrate provided to the microbiota and its origin (i.e., lean or obese individuals).

\section{Acknowledgments}

The authors thank Wendy Borst for assistance in getting acquainted with TIM-2 as well as Tom Gorissen and Hans Kooijman for helping with SCFA and BCFA analyses. 


\section{References}

1. Bray GA (2004) Medical consequences of obesity. J Clin Endocrinol Metab 89: 2583-2589.

2. World Health Organization (WHO) (2012) Obesity and overweight. Fact sheet No311. http://www.who.int/mediacentre/factsheets/fs311/en/index.html

3. Bäckhed F, Manchester JK, Semenkovich CF, Gordon JI (2007) Mechanisms underlying the resistance to diet-induced obesity in germ-free mice. Proc Natl Acad Sci U S A 104: 979-984.

4. Ley RE, Backhed F, Turnbaugh P, Lozupone C.A., Knight R.D., et al. (2005) Obesity alters gut microbial ecology. Proc Natl Acad Sci U S A 102: 1107011075 .

5. Duncan SH, Lobley G.E, Holtrop G, Ince J, Johnstone A.M, et al. (2008) Human colonic microbiota associated with diet, obesity and weight loss. Int J Obes 32: 1720-1724.

6. Venema K (2010) Role of gut microbiota in the control of energy and carbohydrate metabolism. Curr Opin Clin Nutr Metab Care 13: 432-438.

7. Schwiertz A, Taras D, Schäfer K, Beijer S, Bos NA, et al. (2010) Microbiota and SCFA in lean and overweight healthy subjects. Obesity (Silver Spring) 18: 190-195.

8. Bäckhed F, Hao D, Wang T, V. HL, Koh GY, et al. (2004) The gut microbiota as an environmental factor that regulates fat storage. Proc Natl Acad Sci U S A 101: 15718-15723.

9. Bernalier-Donadille A (2010) Fermentative metabolism by the human gut microbiota. Gastroenterol Clin Biol 34, Supplement 1: S16-S22.

10. Flint HJ, Duncan SH, Scott KP, Louis P (2007) Interactions and competition within the microbial community of the human colon: links between diet and health. Environ Microbiol 9: 1101-1111.

11. Bergman EN (1990) Energy contributions of volatile fatty acids from the gastrointestinal tract in various species. Physiol Rev 70: 567-590.

12. Ferchaud-Roucher V, Pouteau E, Piloquet H, Zair Y, Krempf M (2005) Colonic fermentation from lactulose inhibits lipolysis in overweight subjects. Am $J$ Physiol Endocrinol Metab 289: E716-E720.

13. Al-Lahham SH, Roelofsen H, Priebe M, Weening D, Dijkstra M, et al. (2010) Regulation of adipokine production in human adipose tissue by propionic acid. Eur J Clin Invest 40: 401-407.

14. Al-Lahham S, Roelofsen H, Rezaee F, Weening D, Hoek A, et al. (2011) Propionic acid affects immune status and metabolism in adipose tissue from overweight subjects. Eur J Clin Invest 42: 357-364.

15. Roediger WE (1982) Utilization of nutrients by isolated epithelial cells of the rat colon. Gastroenterology 83: 424-429. 
Lean vs Obese: apple fiber, sugar beet, GOS, lactulose fermentation

16. Scheppach W (1994) Effects of short chain fatty acids on gut morphology and function. Gut 35: S35-S38.

17. Gamet L, Daviaud D, Denis-Pouxviel C, Remesy C, Murat J-C (1992) Effects of short-chain fatty acids on growth and differentiation of the human coloncancer cell line HT29. Int J Cancer 52: 286-289.

18. Gibson PR, Moeller I, Kagelari O, Folino M, Young GP (1992) Contrasting effects of butyrate on the expression of phenotypic markers of differentiation in neoplastic and non-neoplastic colonic epithelial cells in vitro. J Gastroenterol Hepatol 7: 165-172.

19. Petersen C (2005) D-lactic acidosis. Nutr Clin Pract 20: 634-645.

20. Duncan SH, Louis P, Flint HJ (2004) Lactate-Utilizing Bacteria, Isolated from Human Feces, That Produce Butyrate as a Major Fermentation Product. Appl Environ Microbiol 70: 5810-5817.

21. Scott KP, Gratz SW, Sheridan PO, Flint HJ, Duncan SH (2013) The influence of diet on the gut microbiota. Pharmacol Res 69: 52-60.

22. Fredstrom SB, Lampe JW, Jung H-JG, Slavin JL (1994) Apparent Fiber Digestibility and Fecal Short-Chain Fatty Acid Concentrations With Ingestion of Two Types of Dietary Fiber. JPEN J Parenter Enteral Nutr 18: 14-19.

23. Gibson GR, Roberfroid MB (1995) Dietary Modulation of the Human Colonic Microbiota: Introducing the Concept of Prebiotics. J Nutr 125: 1401-1412.

24. Playne MJ, Crittenden RG, McSweeney P, Fox PF (2009) Galacto-oligosaccharides and Other Products Derived from Lactose. In: McSweeney PLHF, Patrick F., editor. Advanced Dairy Chemistry. 3rd ed. ed. New York: Springer: Springer New York. pp. 121-201.

25. Sarbini SR, Kolida S, Gibson GR, Rastall RA (2013) In vitro fermentation of commercial alpha-gluco-oligosaccharide by faecal microbiota from lean and obese human subjects. Br J Nutr 109: 1980-1989.

26. Beards E, Tuohy K, Gibson G (2010) Bacterial, SCFA and gas profiles of a range of food ingredients following in vitro fermentation by human colonic microbiota. Anaerobe 16: 420-425.

27. Rowland IR, Tanaka R (1993) The effects of transgalactosylated oligosaccharides on gut flora metabolism in rats associated with a human faecal microflora. $J$ Appl Bacteriol 74: 667-674.

28. Bouhnik Y, Flourié B, D'Agay-Abensour L, Pochart P, Gramet G, et al. (1997) Administration of Transgalacto-Oligosaccharides Increases Fecal Bifidobacteria and Modifies Colonic Fermentation Metabolism in Healthy Humans. J Nutr 127: 444-448.

29. Maathuis AJH, van den Heuvel EG, Schoterman MHC, Venema K (2012) GalactoOligosaccharides Have Prebiotic Activity in a Dynamic in vitro Colon Model Using a C-Labeling Technique. J Nutr 142: 1205-1212. 
30. Van den Heuvel EG, Muijs T, Van Dokkum W, Schaafsma G (1999) Lactulose stimulates calcium absorption in postmenopausal women. $J$ Bone Miner Res 14: 1211-1216.

31. Schwartz SE, Levine RA, Singh A, Scheidecker JR, Track NS (1982) Sustained pectin ingestion delays gastric emptying. Gastroenterology 83: 812-817.

32. Flourie B, Vidon N, Chayvialle JA, Palma R, Franchisseur C, et al. (1985) Effect of increased amounts of pectin on a solid-liquid meal digestion in healthy man. Am J Clin Nutr 42: 495-503.

33. Shinohara K, Ohashi Y, Kawasumi K, Terada A, Fujisawa T (2010) Effect of apple intake on fecal microbiota and metabolites in humans. Anaerobe 16: 510-515.

34. Parkar SG, Redgate EL, Wibisono R, Luo X, Koh ETH, et al. (2010) Gut health benefits of kiwifruit pectins: Comparison with commercial functional polysaccharides. J Funct Foods 2: 210-218.

35. Fukunaga T, Sasaki M, Araki Y, Okamoto T, Yasuoka T, et al. (2003) Effects of the soluble fibre pectin on intestinal cell proliferation, fecal short chain fatty acid production and microbial population. Digestion 67: 42-49.

36. Minekus M, Smeets-Peeters M, Bernalier A, Marol-Bonnin S, Havenaar R, et al. (1999) A computer-controlled system to simulate conditions of the large intestine with peristaltic mixing, water absorption and absorption of fermentation products. Appl Microbiol Biotechnol 53: 108-114.

37. Gibson GR, Cummings J.H., Macfarlane G.T. (1988) Use of a three-stage continuous culture system to study the effect of mucin on dissimilatory sulfate reduction and methanogenesis by mixed populations of human gut bacteria. Appl Environ Microbiol 54: 2750-2755.

38. Duncan SH, Scott KP, Ramsay AG, Harmsen HJM, Welling GW, et al. (2003) Effects of Alternative Dietary Substrates on Competition between Human Colonic Bacteria in an Anaerobic Fermentor System. Appl Environ Microbiol 69: 1136-1142.

39. Lawson DJ, Holtrop G, Flint H (2011) Bayesian analysis of non-linear differential equation models with application to a gut microbial ecosystem. Biom $J$ 53: 543-556.

40. Venema K, Van Nuenen HMC, Smeets-Peeters M, Minekus M, Havenaar R (2000) TNO's in vitro large intestinal model: an excellent screening tool for functional food and pharmaceutical research. Ernährung/Nutrition 24: 558564.

41. Aguirre M, Ramiro-Garcia J, Koenen ME, Venema K (2014) To pool or not to pool? Impact of the use of individual and pooled fecal samples for in vitro fermentation studies. J Microbiol Methods 107C: 1-7.

42. Maathuis A, Hoffman A, Evans A, Sanders L, Venema K (2009) The Effect of the Undigested Fraction of Maize Products on the Activity and Composition of the 
Lean vs Obese: apple fiber, sugar beet, GOS, lactulose fermentation

Microbiota Determined in a Dynamic in vitro Model of the Human Proximal Large Intestine. J Am Coll Nutr 28: 657-666.

43. De Graaf AA, Maathuis A, De Waard P, Deutz NEP, Dijkema C, et al. (2010) Profiling human gut bacterial metabolism and its kinetics using $\left[\mathrm{U}^{13} \mathrm{C}\right]$ glucose and NMR NMR Biomed 23: 2-12.

44. Van Nuenen HMC, Meyer P.D., Venema K (2003) The effect of various inulins and Clostridium difficile on the metabolic activity of the human colonic microbiota in vitro. Microb Ecol Health Dis 15: 137-144.

45. Van der Kamp JW, Jones J, Mccleary B, Topping DJ (2010) Dietary Fibre: New Frontiers for Food and Health: Wageningen Academic Publishers.

46. Marino PL, Sutin KM (2012) The ICU Book: Wolters Kluwer Health, Philadelphia, USA.

47. Schloss PD, Westcott SL, Ryabin T, Hall JR, Hartmann M, et al. (2009) Introducing mothur: open-source, platform-independent, communitysupported software for describing and comparing microbial communities. Appl Environ Microbiol 75: 7537-7541.

48. Roeselers G, Mittge EK, Stephens WZ, Parichy DM, Cavanaugh CM, et al. (2011) Evidence for a core gut microbiota in the zebrafish. Isme J 5: 1595-1608.

49. Huse SM, Welch DM, Morrison HG, Sogin ML (2010) Ironing out the wrinkles in the rare biosphere through improved OTU clustering. Environ Microbiol 12: 1889-1898.

50. Pruesse E, Quast C, Knittel K, Fuchs BM, Ludwig W, et al. (2007) SILVA: a comprehensive online resource for quality checked and aligned ribosomal RNA sequence data compatible with ARB. Nucleic Acids Res 35: 7188-7196.

51. Edgar RC, Haas BJ, Clemente JC, Quince C, Knight R (2011) UCHIME improves sensitivity and speed of chimera detection. Bioinformatics 27: 2194-2200.

52. Wang Q, Garrity GM, Tiedje JM, Cole JR (2007) Naive Bayesian Classifier for Rapid Assignment of rRNA Sequences into the New Bacterial Taxonomy. Appl Environ Microbiol 73: 5261-5267.

53. Hamady M, Lozupone C, Knight R (2010) Fast UniFrac: facilitating highthroughput phylogenetic analyses of microbial communities including analysis of pyrosequencing and PhyloChip data. ISME J 4: 17-27.

54. Kovatcheva-Datchary P, Egert M, Maathuis A, Rajilic -Stojanovic M, De Graaf AA, et al. (2009) Linking phylogenetic identities of bacteria to starch fermentation in an in vitro model of the large intestine by RNA-based stable isotope probing. Environ Microbiol 11: 914-992.

55. Van den Abbeele P, Venema K, Van de Wiele T, Verstraete W, Possemiers S (2013) Different Human Gut Models Reveal the Distinct Fermentation Patterns of Arabinoxylan versus Inulin. J Agric Food Chem 61: 9819-9827.

56. Venema K (2012) Intestinal fermentation of lactose and prebiotic lactose derivatives, including human milk oligosaccharides. Int Dairy J 22: 123-140. 
57. Macfarlane S, Macfarlane GT (2003) Regulation of short-chain fatty acid production. Proc Nutr Soc 62: 67-72.

58. Morrison DJ, Mackay WG, Edwards CA, Preston T, Dodson B, et al. (2006) Butyrate production from oligofructose fermentation by the human faecal flora: what is the contribution of extracellular acetate and lactate? $\mathrm{Br} J$ Nutr 96: $570-577$.

59. Hamer HM, Jonkers D, Venema K, Vanhoutvin S, Troost FJ, et al. (2008) Review article: the role of butyrate on colonic function. Aliment Pharmacol Ther 27: 104-119.

60. Salvador V, Cherbut C, Barry JL, Bertrand D, Bonnet C, et al. (1993) Sugar composition of dietary fibre and short-chain fatty acid production during in vitro fermentation by human bacteria. Br J Nutr 70: 189-197.

61. Cummings JH, Beatty ER, Kingman SM, Bingham SA, Englyst HN (1996) Digestion and physiological properties of resistant starch in the human large bowel. Br J Nutr 75: 733-747.

62. Payne AN, Chassard C, Banz Y, Lacroix C (2012) The composition and metabolic activity of child gut microbiota demonstrate differential adaptation to varied nutrient loads in an in vitro model of colonic fermentation. FEMS Microbiol Ecol 80: 608-623.

63. Russell DA, Ross RP, Fitzgerald GF, Stanton C (2011) Metabolic activities and probiotic potential of bifidobacteria. Int J Food Microbiol 149: 88-105.

64. McBain AJ, Macfarlane GT (1998) Ecological and physiological studies on large intestinal bacteria in relation to production of hydrolytic and reductive enzymes involved in formation of genotoxic metabolites. J Med Microbiol 47: 407-416.

65. Kageyama A, Benno Y (2000) Catenibacterium mitsuokai gen. nov., sp. nov., a gram-positive anaerobic bacterium isolated from human faeces. Int $J$ Syst Evol Microbiol 50: 1595-1599.

66. Brook I (2008) Microbiology and management of abdominal infections. Dig Dis Sci 53: 2585-2591.

67. Libby DB, Bearman G (2009) Bacteremia due to Clostridium difficille-review of the literature. Int J Infect Dis 13: e305-e309.

68. Neyrinck AM, Possemiers S, Druart C, Van de Wiele T, De Backer F, et al. (2011) Prebiotic effects of wheat arabinoxylan related to the increase in bifidobacteria, Roseburia and Bacteroides/Prevotella in diet-induced obese mice. PLoS One 6: 9.

69. Sokol H, Pigneur B, Watterlot L, Lakhdari O, G. B-HL, et al. (2008) Faecalibacterium prausnitzii is an anti-inflammatory commensal bacterium identified by gut microbiota analysis of Crohn disease patients. Proc Natl Acad Sci U S A 105: 16731-16736. 
Lean vs Obese: apple fiber, sugar beet, GOS, lactulose fermentation

70. Furet J-P, Kong L-C, Tap J, Poitou C, Basdevant A, et al. (2010) Differential adaptation of human gut microbiota to bariatric surgery-induced weight loss: links with metabolic and low-grade inflammation markers. Diabetes 59: 30493057. 


\section{Supplemental material}

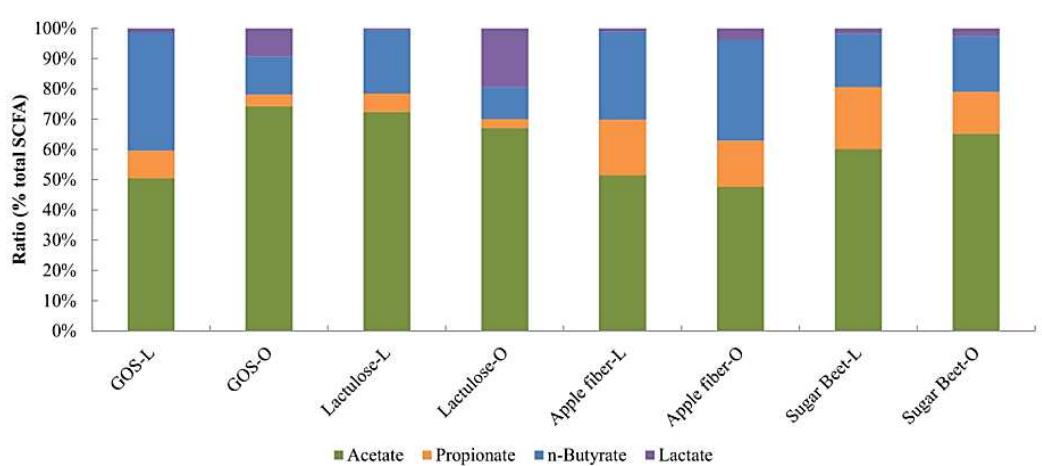

Fig. S1. Averaged molar ratios for acetate, propionate, $n$-butyrate and lactate (\% of total SCFA).

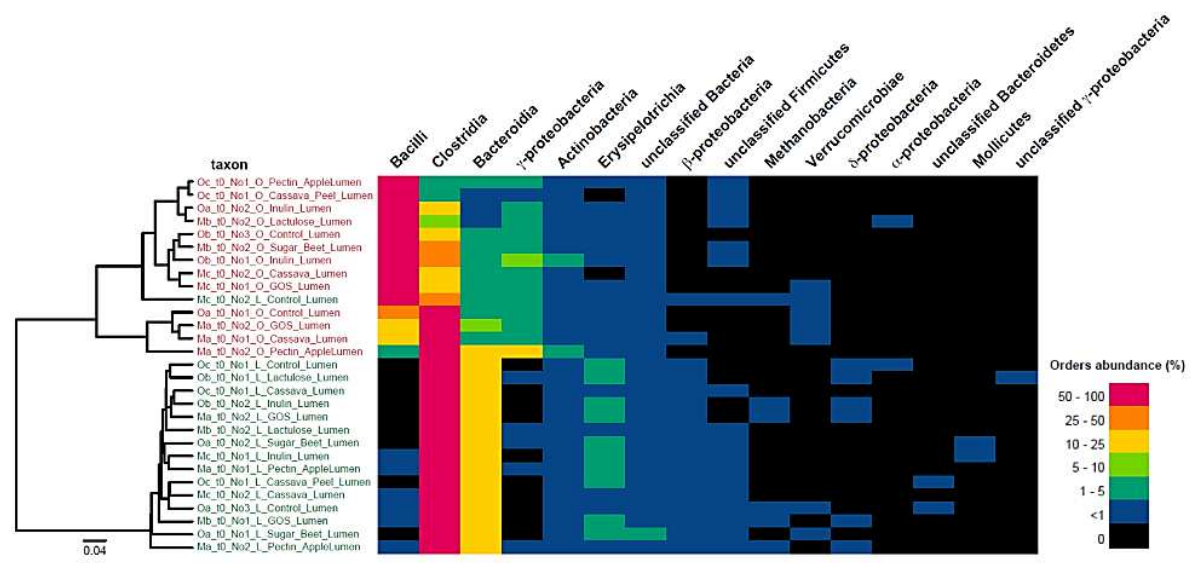

Fig. S2. (A) Weighted UniFrac tree of 16S rDNA pyrosequences. Spanning of the V5-V7 hypervariable regions derived from the different TIM microbiotas (green for L; red for O). Data shown corresponds to a single run after the adaptation period without the addition of the test compound. Scale bars indicate distance between the samples in UniFrac units. (B) The relative abundance of bacterial orders observed in these data sets is represented in a heatmap, showing those bacterial groups that contribute largely to the difference between the two clusters. 


\section{Chapter 7}

The gut microbiota from lean and obese subjects contribute differently to the fermentation of arabinogalactan and inulin

Aguirre M, Bussolo de Souza C, Venema K Submitted 


\section{Abstract}

An aberrant metabolic activity or a compositional alteration of the gut microbiota has been proposed as a factor that makes us more prone to disease. Therefore, we explored the effect of two dietary fibers (arabinogalactan and inulin) on the microbiota from lean and obese subjects during $72 \mathrm{~h}$ in vitro fermentation experiments using the validated TNO dynamic in vitro model of the proximal colon: TIM-2.

Metabolically, arabinogalactan fermentation showed a higher production of propionate when compared to $n$-butyrate in the obese microbiota fermentations. In general, lean microbiota produced more $n$-butyrate from the fermentation of both substrates when compared to the obese microbiota. Furthermore, the obese microbiota extracted more energy from the fermentation of both fibers.

Compositionally, bacteria belonging to Gemmiger, Dorea, Roseburia, Alistipes, Lactobacillus and Bifidobacterium genera were found to be highly abundant or stimulated by the prebiotics in the lean microbiota suggesting a potential role in leanness. Furthermore, a significant correlation between known butyrogenic strains including $B$. adolescentis, an unclassified Bifidobacterium and $F$. prausnitzii with this metabolite in the fermentation of inulin in both microbiotas was found.

Although supplementary in vivo studies are needed, the current study provides more evidence for the consumption of specific ingredients with the aim of modulating the gut microbiota in the context of obesity. 
Lean vs Obese: arabinogalactan, inulin fermentation

\section{Introduction}

The discovery of the potential impact of the gut microbiota on human health and disease has fuelled research on characterizing the role that this community plays in the causality or prevention of many diseases elicited by dangerous lifestyles such as sedentary and bad eating habits, among others [1,2].

Part of the efforts have been focused on identifying a balanced and thus, healthy community [3]. Though provocative, it is difficult to define a "most desirable" composition for the human gut microbiota. Reports providing contradictory findings, due to either i) a large inter-individual variation or ii) the application of different analytic methods, are at the order of the day. However, another factor which seems to play an important role in influencing health and disease, besides the community composition, is the interaction of the microbial metabolites with the host.

The fermentation of dietary fiber by the gut microbiota leads primarily to the production of short-chain fatty acids (SCFA; mainly acetate, propionate and butyrate) and the gases hydrogen, methane and carbon dioxide [4]. Furthermore, branched-chain fatty acids (BCFA; mainly iso-butyrate and iso-valerate often accompanied by phenol and ammonia production) are also produced to a lesser extent but these originate from protein fermentation [5]. A proposed mechanism by which fiber may protect us against obesity is based on the beneficial effects that such metabolites have on host energy balance, e.g. by mediating the secretion of gut hormones involved in the regulation of energy metabolism and food intake (including leptin, peptide YY and glucagon-like peptide-1) [6,7]. Thus, it may be tempting to say that high intake of fiber would be a way to reduce the risk of obesity [8,9]. After all, it is estimated that the production of SCFA by the microbiota accounts for 5 to $10 \%$ of total dietary energy requirements in humans [10]. However, recent research has questioned such risk-reduction role. There is growing evidence indicating that the production of SCFA differs between the microbiota originating from obese and lean individuals (hereafter referred to as obese and lean microbiota). Such difference lies in the fact that the obese microbiota may produce more SCFA which could be translated into more energy extraction from diet $[11,12]$. As a consequence, more energy extracted from diet may be stored as fat, promoting host's weight gain. Such mechanisms place fiber fermentation by the gut microbiota as a causative factor in obesity. Still, as previously remarked, there is a lack of consistency, different studies show contrasting results by finding either no correlation between fiber and weight gain/obesity, a reverse trend or effects to be substrate dependent [12-14]. 
Vast amounts of research are needed to answer the chicken or the egg causality dilemma before any strategy can be designed with the aim of manipulating the gut microbiota in the context of obesity. Currently, there is a limited number of in vitro fermentation experiments mimicking the fermentation of different substrates by human obese or lean microbiota. So far, these studies have provided evidence about the metabolic adaptation of the microbiota in relation to different nutrient loads or single testing of specific prebiotics, as well as the plasticity of the microbiota in configuring the structure of the community in response to this kind of interventions [14-19]. Importantly, these studies have also endorsed in vitro systems as tools facilitating the medium to high-throughput validation of multiple hypotheses at lower costs with no ethical constraints when compared to human or animal studies.

The purpose of the current study was to compare the profiles of fermentation of arabinogalactan (AG) and the well-studied prebiotic inulin (IN) by obese or lean microbiota. Both AG and IN are natural polysaccharides commonly found in foods. They have been found to be fermented by human intestinal bacteria and stimulate the production of SCFA and the growth of specific bacteria generally believed to be beneficial to the host [20-22]. AG is an interesting compound to evaluate not only because its potential to improve gut barrier function [23], but also because it has been observed that it may induce production of (both pro and antiinflammatory) cytokines [24,25], factors which both may play an important role in inflammation. In the context of obesity, low grade inflammation has been suggested to contribute to the development of insulin and leptin resistance [26].

In order to compare the two prebiotics, we performed $72 \mathrm{~h}$ fermentation experiments in the validated TNO dynamic in vitro model of the proximal colon (TIM-2), which was inoculated with either obese or lean microbiota. The present work provides evidence about how fermentable carbohydrates are differently used by the microbiota from lean and obese subjects which contributes to the understanding on how dietary compounds could be used as therapeutic tools in obesity.

\section{Materials and Methods}

\section{Gut microbiota}

The inocula used for the TIM-2 experiments consisted of an active, pooled fecal microbiota prepared from: i) 8 healthy lean volunteers (male: $n=4$, female: $n=4$, average age $=31$ y (range: $25-42$ ), $\mathrm{BMI}=20 \pm 1.48 \mathrm{~kg} / \mathrm{m}^{2}$ ); ii) 7 healthy obese volunteers (male: $\mathrm{n}=3$, female: $\mathrm{n}=4$, average age $=51$ y (range: $29-68$ ), $\mathrm{BMI}=32 \pm$ $\left.1.17 \mathrm{~kg} / \mathrm{m}^{2}\right)$. We have previously shown that pooling does not result in an aberrant 
microbiota composition or activity [27]. The exclusion criteria for lean and obese volunteers included the use of antibiotics during the preceding 3 months, gastrointestinal disease, severe chronic disease or food allergy and intake of probiotics and prebiotics.

Whole fecal samples were self-collected in a container kit which was maintained under anaerobiosis by using anaerobic packs (AnaeroGen ${ }^{\mathrm{TM}}$, Oxoid, Cambridge, UK). A sample aliquot $(100 \mathrm{mg}$ ) from each individual donation was collected in an anaerobic cabinet $\left(80 \% \mathrm{~N}_{2}, 10 \% \mathrm{CO}_{2}, 10 \% \mathrm{H}_{2}\right)$, snap-frozen in liquid nitrogen ($196{ }^{\circ} \mathrm{C}$ ) and stored at $-80{ }^{\circ} \mathrm{C}$ for measurement of metabolites (SCFA and BCFA). Feces were homogenized under anaerobic conditions as described by Aguirre et al. [28]. The resulting culture homogenate was aliquoted and snap-frozen in liquid nitrogen. This microbiota was stored at $-80{ }^{\circ} \mathrm{C}$ before inoculation in TIM-2.

\section{Gut fermentation experiments}

The TIM-2 system was flushed with $\mathrm{N}_{2}$ prior to the introduction of the inoculum for $3 \mathrm{~h}$ and it was maintained under this condition at $37{ }^{\circ} \mathrm{C}$ for $96 \mathrm{~h}$ with the $\mathrm{pH}$ kept at or above 5.8 by automatic titration with $2 \mathrm{M} \mathrm{NaOH}$. A $30 \mathrm{ml}$ portion of culture homogenate was used to inoculate the units for each experiment. The microbiota was left to adapt $(16 \mathrm{~h})$ to the new environment after inoculation and during this period the basal simulated ileal efflux medium (SIEM) was gradually introduced into the system in a total volume of $40 \mathrm{ml}$. After the adaptation, the culture was deprived from any medium for $2 \mathrm{~h}$ (starvation). A volume of $180 \mathrm{ml}$ of the different diets and control was administrated over the $72 \mathrm{~h}$ of the test period at a rate of $2.5 \mathrm{ml} / \mathrm{h}$.

In order to remove water and fermentation products from the lumen, a dialysate system (described in detail by van Nuenen et al. [29]), consisting of a semipermeable hollow membrane, ran through the lumen. For all the experiments, the speed of the dialysis fluid was set at $1.5 \mathrm{ml} / \mathrm{min}$.

After 24 and $48 \mathrm{~h}$ of fermentation $25 \mathrm{ml}$ of lumen sample was removed from the system to mimic the transit of material from the proximal and reaching the distal colon [30]. Luminal and dialysate samples were taken after $\mathrm{t}=0,24,48$ and $72 \mathrm{~h}$. In all cases samples were snap-frozen in liquid nitrogen and stored $\left(-80{ }^{\circ} \mathrm{C}\right)$ until analysis.

Fermentation media

During the adaptation period ( $16 \mathrm{~h})$ all TIM-2 units were fed with SIEM as described by Maathuis et al.[31]. After this adaptation period and the $2 \mathrm{~h}$ starvation period, the units were fed with preparations which were made containing approximately $7.5 \mathrm{~g}$ of AG or IN instead of the standard carbohydrates in SIEM. 
The specific AG used in this study was (+)-Arabinogalactan- from larch wood (Sigma-Aldrich, St Louis, USA) with a molecular weight ranging from 72-92 kDa and $\geq 84.8 \%$ purity. The IN tested had degree of polymerization (DP) of 9; 84.9\% $>$ DP5 (Sensus, Frutafit ${ }^{\circledR}$ IQ, Roosendaal, The Netherlands). Control units were fed with SIEM.

Analysis of SCFA (acetate, propionate, and $n$-butyrate) and BCFA (iso-butyrate and iso-valerate)

Samples were prepared and analyzed as described previously [14]. Before centrifuging, the fecal aliquots from the individuals were suspended in PBS (1:1; w:w). Briefly, both suspended aliquots and TIM-2 luminal samples were centrifuged (12000 r.p.m. at $4{ }^{\circ} \mathrm{C}$ for $10 \mathrm{~min}$ ). To the clear supernatant a mixture of formic acid (20\%), methanol and 2-ethyl butyric acid (internal standard, $2 \mathrm{mg} / \mathrm{ml}$ in methanol) was added. A $3 \mu \mathrm{l}$ sample with a split ratio of 75.0 was injected on a GC-column (ZB-5HT inferno, ID $0.52 \mathrm{~mm}$, film thickness $0.10 \mathrm{um}$; Zebron; phenomenex, USA) in a Shimadzu GC-2014 gas chromatograph. Standard curves were obtained by injecting known quantities of a blend of volatile fatty acids and amounts were calculated from the graph obtained correlating peak height and time measured (all reagents from Sigma-Aldrich with the exception of formic acid which was from Merck).

Energy extraction

Energy extraction in the form of SCFA was calculated using the following $\mathrm{kJ} \mathrm{mol}^{-1}$ values for acetate, propionate and $n$-butyrate respectively: 874, 1536 and 2192 $[32,33]$.

Characterization of bacterial populations

RNA was isolated from luminal samples using standard molecular biology kits from ZYMO Research (Zymo Research Co., CA, USA) following manufacturer's instructions. Reverse-transcriptase amplification of the 16S rRNA gene (V3-V4), barcoding and library preparation (1st step PCR and 2nd PCR) were performed by BaseClear, Leiden, The Netherlands.

Short paired-end sequence reads were generated using the Illumina MiSeq system and converted into FASTQ files using the BCL2FASTQ pipeline version 1.8.3. Quality trimming was applied based on Phred quality scores. Subsequently, the Illumina paired reads were merged into single reads (so-called pseudoreads) through sequence overlap (16S rRNA V3-V4 region of about 500bp). Chimeric pseudoreads were removed and the remaining reads were aligned to a combination of the GreenGenes and RDP 16S gene databases [34,35]. Based on the alignment 
scores of the pseudoreads, the taxonomic classes were assigned by associating each pseudoread to the best matching Operational Taxonomic Unit (OTU). The taxonomic depth of the lineage is based on the identity threshold of the rank; Species 99\%, Genus 97\%, Family 95\%, Order 90\%, Class 85\%, Phylum 80\%.

Data analysis

The experiments were performed in series of two per tested substrate $(n=2)$. To avoid unnecessary repetition, this is not indicated further in the text or graphs in the results section. Results are displayed as average of these duplicates. For simplicity of reading, substrates in the following sections are tagged with the letter $\mathrm{L}$ or $\mathrm{O}$ (e.g. substrate-L, substrate-O) in order to refer to the fermentation experiments using the inoculum from lean (-L) or obese (-O) subjects.

Statistics analyses for determining the differences in metabolite production and energy extraction in the fecal samples from each individual were performed using Microsoft Excel (2010). Comparison between the two groups (lean and obese) was performed using t-test with significance $\mathrm{p}<0.05$.

For the calculation of fold compositional changes the ratio between a sampling time point and $t 0$ was calculated (i.e., $\mathrm{t} 72 / \mathrm{t} 0$ ). The ratio for this value and the control was then determined to obtain fold changes. A value equal to 1 indicates no change; a value of $>1$ indicates an increase; and a value of $<1$ indicates a decrease of the respective microbial taxa.

Differentially abundant bacterial species growing on the different tested substrates were calculated from a ratio based from the specific growth of the species found on each substrate and the control. Spearman correlations were calculated between the ratio of the species identified as being differentially abundant and the measured amounts of metabolites produced (SPSS for Windows, version 21, SPSS, Chicago, US). Equal variance was assumed and calculations were performed from two-tailed distribution. Correlations were considered significant at the 0.01 level (2-tailed).

\section{Ethics Statement}

Studies using fecal donations from healthy volunteers do not require medical ethical committee approval in The Netherlands since they are considered as noninvasive. However, volunteers who donated the inoculum were informed prior to initiating the study and their participation was considered after providing a signed informed consent. The group of obese donors was recruited at Maastricht University Medical Center (The Netherlands). These were patients from the university medical center who voluntarily responded to a recruiting call for inviting to donate their feces. The group of lean donors was recruited at TNO (The Netherlands). These participants responded to an advertisement inviting subjects to 
voluntarily collect their feces. Bouke Salden and Carlota Bussolo de Souza personally collected the fecal samples from the participants who exclusively donated their feces for the present study. Bouke Salden received the fecal samples directly from the obese participants and none of the authors was involved in the direct collection of these samples. Carlota Bussolo de Souza received the fecal samples directly from the lean participants. The origins of the both lean and obese fecal donations were blinded using a code whose identity was known only by the responsible scientist (Carlota Bussolo de Souza, co-author of the present study). Results in this manuscript are referred to an individual sample or a pooled fecal inoculum and do not directly refer to a particular person.

\section{Results}

Screening of metabolites and energy extraction in feces from the volunteers

The average amount of SCFA found in the feces from lean and obese subjects were not statistically different $(259.6 \pm 100.2 \mathrm{mmol}$ and $215.1 \pm 66.9 \mathrm{mmol}$, respectively). However, it was clear that there was a great inter-individual difference regarding the amount of each SCFA in both groups (Table S1). When comparing the amount of each SCFA and the amount of energy content, no statistically significant differences were found.

BCFA were higher in the feces from lean volunteers $(p<0.05)$. On average, feces from lean subjects contained $7.88 \pm 2.54 \mathrm{mmol}$ of iso-butyrate and $11.45 \pm 3.64$ mmol of $i$-valerate, while feces from obese subjects contained on average $3.11 \pm$ $1.98 \mathrm{mmol}$ of $i$-butyrate and $4.85 \pm 2.92 \mathrm{mmol}$ of $i$-valerate. BCFA amounts also presented a great inter-individual difference among subjects.

Fermentation experiments with lean and obese microbiota

Microbial activity

Total SCFA production was higher in fermentations using the obese microbiota when compared to the lean (Fig 1). Fermentation kinetics in terms of SCFA production observed from both inocula differed in AG, IN and control experiments. Fermentation of AG showed major differences in propionate and $n$-butyrate production compared to the other fermentations, with propionate even higher than $n$-butyrate in the obese microbiota fermentations. In general, $n$-butyrate production is higher in the lean fermentations for all substrates when compared to obese, while propionate is observed to be higher in the fermentations with the obese microbiota when compared to lean. 

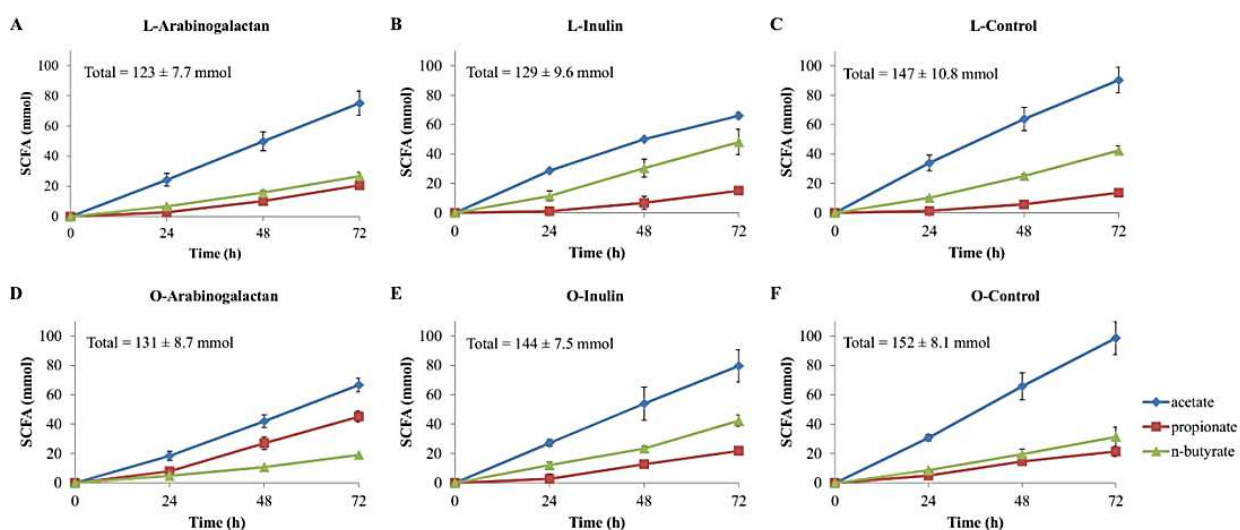

Fig. 1. Cumulative production of SCFA (mmol) during the $72 \mathrm{~h}$ of fermentation of the tested substrates.

Table 1 shows the cumulative production of BCFA after $72 \mathrm{~h}$ of fermentation of AG, IN and control. Lean microbiota produced more BCFA from AG fermentation and less from IN than control. For AG this was also observed in the obese microbiota. The values from the obese fermentations were lower when compared to lean for control and AG, but not for IN.

Table 1. Cumulative production of BCFA after $72 \mathrm{~h}$ of fermentation of AG, IN and control.

\begin{tabular}{l|ccc|ccc}
\multirow{2}{*}{$\begin{array}{l}\text { Test } \\
\text { Compound }\end{array}$} & \multicolumn{3}{|c}{ Total cumulative production of BCFA (mmol) by lean and obese microbiota } \\
\cline { 2 - 7 } & $i$-butyrate & $i$-valerate & Total & $i$-butyrate & $i$-valerate & Total \\
\hline Arabinogalactan & $1.62 \pm 0.66$ & $2.32 \pm 0.08$ & $3.94 \pm 0.75$ & $0.55 \pm 0.36$ & $1.59 \pm 0.38$ & $2.13 \pm 0.74$ \\
Inulin & $0.22 \pm 0.2$ & $1.23 \pm 0.15$ & $1.45 \pm 0.35$ & $0.45 \pm 0.07$ & $1.49 \pm 0.08$ & $1.94 \pm 0.15$ \\
Control & $0.74 \pm 0.14$ & $1.99 \pm 0.4$ & $2.73 \pm 0.27$ & $0.44 \pm 0.28$ & $1.22 \pm 0.06$ & $1.66 \pm 0.22$
\end{tabular}

\section{Energy extraction}

The microbiota from obese volunteers fermenting AG and IN extracted (slightly) more energy when compared to the lean fermentations, in accordance with the higher SCFA production. Controls remained quite similar with respect to energy extraction (Fig 2). 


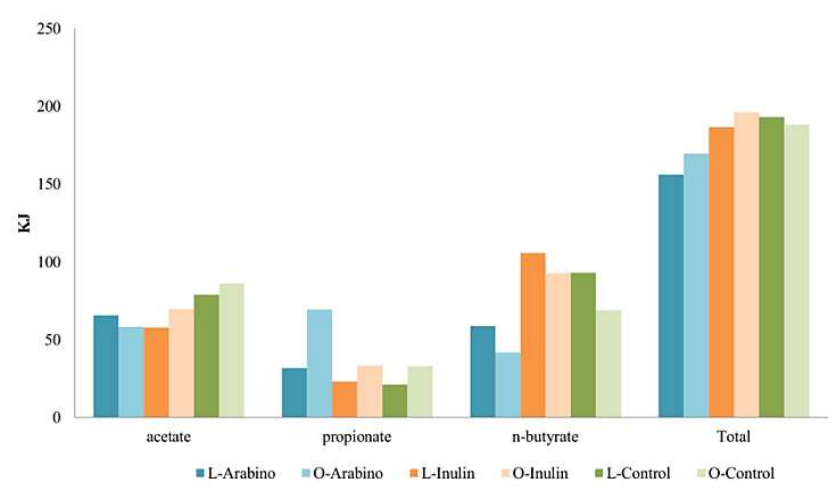

Fig. 2. Energy extraction. Values for the individual SCFA and the sum (total) obtained after $72 \mathrm{~h}$ fermentation experiments of AG, IN and control using lean or obese microbiota.

\section{Compositional changes}

At genus level, it was observed that differences among the inocula are driven by the abundances within the same groups of bacteria (Fig S1): the major groups in the lean microbiota were Bifidobacterium, Clostridium, Lactobacillus and Enterococcus (23, 34, 14 and 13\%, respectively) and in the obese microbiota were also Clostridium, Lactobacillus, Enterococcus, Bifidobacterium but at different abundances (46, 17, 15 and 10\%).

Analysis of the species found in the inoculum ( $\mathrm{t} 0$ ) suggests that the lean microbiota has a more diverse population of Bifidobacterium which included B. adolescentis, B. longum and an unclassified group (7, 4 and $8 \%$, respectively) whilst the obese microbiota mainly contains B. longum and an unclassified group (6 and 3\%, respectively). Both microbiotas share a high abundance of $C$. butyricum (lean: 23\%; obese: $30 \%$ ).

By calculating L/O ratios (at the genus level), it was found that the lean microbiota had an increased relative abundance of Faecalibacterium (75 fold), Dorea (30 fold), Roseburia (6 fold), Blautia ( 3 fold) when compared to the obese microbiota at t0 (Table S2; for more information about the starting inocula refer to supplemental material Fig. S1).

The fermentation of AG and IN resulted in the growth or decrease of specific genera. Tables 2 (AG) and 3 (IN) show the effects observed in the different microbiotas per substrate tested, compared to the control. 
Table 2. Relative change of bacterial genera after $72 \mathrm{~h}$ of fermentation experiments of arabinogalactan in TIM-2 compared to control.

\begin{tabular}{|l|c|c|} 
& \multicolumn{2}{|c|}{ Arabinogalactan } \\
Genus & Lean & Obese \\
\hline Lactobacillus & 0.49 & 2.56 \\
\hline Enterococcus & 0.51 & 1.65 \\
\hline Unclassified & 0.99 & 2.24 \\
\hline Escherichia & 1.11 & 0.98 \\
\hline unclassified Clostridiaceae & 0.37 & 1.29 \\
\hline unclassified Lactobacillaceae & 0.25 & 3.70 \\
\hline unclassified Enterococcaceae & 0.55 & 1.39 \\
\hline Shigella & 0.57 & 1.57 \\
\hline Faecalibacterium & 0.35 & 1.53 \\
\hline Bacteroides & 1.33 & 20.47 \\
\hline Eubacterium & 0.54 & 2.95 \\
\hline unclassified Enterobacteriaceae & 2.78 & 1.18 \\
\hline unclassified Eubacteriaceae & 0.86 & 137.78 \\
\hline Weissella & 74.12 & 0.00 \\
\hline Collinsella & 1.63 & 0.01 \\
\hline Pseudomonas & 0.88 & 1.34 \\
\hline Blautia & 0.84 & 1.95 \\
\hline Turicibacter & 0.21 & 1.58 \\
\hline Dorea & 0.95 & 14.16 \\
\hline unclassified Bacteroidaceae & 1.19 & 13.97 \\
\hline Fusicatenibacter & 0.03 & 16.98 \\
\hline Parabacteroides & 0.31 & 10.11 \\
\hline unclassified Peptostreptococcaceae & 0.65 & 3.22 \\
\hline & & \\
\hline
\end{tabular}

Table 3. Relative change of bacterial genera after $72 \mathrm{~h}$ of fermentation experiments of inulin in TIM-2 compared to control.

\begin{tabular}{|l|c|c|} 
& \multicolumn{2}{|c|}{ Inulin } \\
Genus & Obese \\
\hline Bifidobacterium & 0.77 & 1.96 \\
\hline Lactobacillus & 0.15 & 1.08 \\
\hline Unclassified & 0.83 & 2.57 \\
\hline $\begin{array}{l}\text { unclassified } \\
\text { Bifidobacteriaceae }\end{array}$ & 1.09 & 1.83 \\
\hline unclassified Lactobacillaceae & 0.15 & 1.57 \\
\hline Faecalibacterium & 0.97 & 2.70 \\
\hline Bacteroides & 2.84 & 0.89 \\
\hline Collinsella & 1.10 & 0.36 \\
\hline unclassified Ruminococcaceae & 0.98 & 3.27 \\
\hline $\begin{array}{l}\text { unclassified } \\
\text { Coriobacteriaceae }\end{array}$ & 9.06 & 0.69 \\
\hline Blautia & 0.49 & 4.53 \\
\hline unclassified Bacteroidaceae & 2.62 & 0.88 \\
\hline Fusicatenibacter & 0.21 & 27.99 \\
\hline unclassified Lachnospiraceae & 0.73 & 2.50 \\
\hline
\end{tabular}


$\underline{\text { Arabinogalactan }}$

Genera that increased after the fermentation of AG by the obese microbiota but decreased in the experiments with the lean microbiota are the well-studied: Lactobacillus (3 vs 0.49 fold), Dorea (14 vs 0.95 fold), Fusinibacter (17 vs 0.03 fold), Parabacteroides (10 vs 0.31 fold), Faecalibacterium ( 2 vs 0.35 fold), and Blautia (2 vs 0.84 fold). At the species level the growth of B. longum was stimulated in the fermentation with the lean microbiota while it decreased in the obese ( 2 vs 0.21 fold, respectively; Table S3) this effect was opposite for $L$. mисоsae ( 0.50 vs 2 fold, respectively). When compared to the control, B. caccae and $B$. thetaiotaomicron notably increased in the lean microbiota (44 and 7 fold, respectively) whilst the growth of $L$. gasseri (12 fold) was stimulated in the fermentations with the obese microbiota.

Inulin

After the fermentation of IN by the obese microbiota an increase in Bifidobacterium (2 vs 0.8 fold), Faecalibacterium (3 vs 0.97 fold), Blautia (5 vs 0.5 fold) and Fusicatenibacter (28 vs 0.2 fold) was observed with respect to the lean microbiota. The fermentation of IN promoted the growth of $B$. adolescentis and an unclassified Bifidobacterium in the obese microbiota when compared to lean (5 vs 0.66 fold; 2 vs 0.76 fold, respectively; Table S3). Enterococcus faecalis was increased in the fermentations with the lean microbiota when compared to control (6 fold).

Modulatory effect of the substrates on activity and composition of the microbiota Species in general were found to be divided in two groups: species that were positively correlated with $n$-butyrate (found in O-inulin and L-inulin experiments) and species that were negatively correlated to acetate, propionate and BCFA production (found in L-Inulin and L-arabino).

More specifically, correlation analysis performed showed a significant positive correlation of $B$. adolescentis, an unclassified Bifidobacterium, $F$. prausnitzii, an unclassified Faecalibacterium and an unclassified Eubacterium with $n$-butyrate production; acetate, propionate and BCFA production were significantly negatively correlated with $F$. prausnitzii and unclassified Faecalibacterium but with an unclassified Bacteroides as well (Fig 3). 

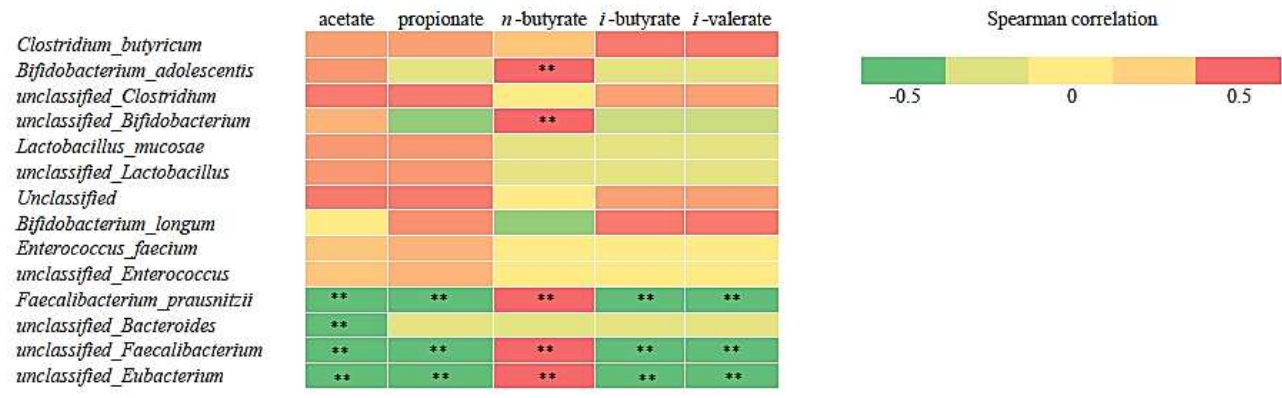

Fig. 3. Correlation of metabolites and a subset of marker bacterial species. Rows correspond to bacterial species; columns correspond to measured metabolites. Red and green denote positive and negative correlation, respectively. The intensity of the colors represents the degree of association between taxa abundances and metabolites as measured by Spearman's correlations. ** indicate associations significant at the 0.01 level (2-tailed).

\section{Discussion}

The addition of fiber to food products has been proposed to reduce the caloric density and glycemic impact of meals [36]. Therefore, there is growing interest in the use of functional fibers in the form of food ingredients, additives or supplements in order to fortify the Western diet without compromising the palatability of the food, especially in long-term weight management programs [36,37].

Other properties of dietary fiber have been well documented and it has been observed that it exerts a wide array of biochemical, neurohormonal and microbiological effects in the human body [36]. Here the gut microbiota, as a metabolic organ, has been found to be influenced by fiber consumption.

The amount and type of dietary fibers consumed have a direct impact on the microbial fermentation capacity [38]. In this respect, studies have found that the gut microbiota from obese subjects could be more efficient in extracting energy from diet than lean subjects $[11,39,40]$. However, findings are controversial and it has been proposed that fermentation of fibers, and consequently their health effects, may be substrate dependent. Substrate dependency could be explained by the fact that dietary fibre reflects a heterogeneous group of compounds that differ in their chemical structure and physico-chemical properties, therefore reflecting on different physiological functions or health benefits [41,42].

In this study, AG and IN were provided to the microbiota from lean or obese subjects and $72 \mathrm{~h}$ fermentation experiments were performed. The aim was to 
compare the differences in the fermentation profiles of these two fibers with respect to microbiota composition, but particularly with respect to production of the microbial metabolites SCFA, which are an energy source for the host.

Determination of fiber fermentation in humans and rats is a common approach to characterize the capacity of the gut microbiota to ferment a specific substrate. However, these studies are expensive, time consuming and in the case of rats the yield of metabolic products from the fermentation of several types of fiber have been found to be significantly lower when compared to humans [43-45]. Moreover, studies in humans are limited because of the limited sampling capacity (noninvasively only feces can be collected), while most of the SCFA produced are taken up during transit of the chyme through the colon (estimated at $95 \%$ of produced SCFA) [46]. Here, we used the TIM-2 system as an alternative tool to animal and human studies. Despite the limitations that in vitro systems carry, such as lack of host interaction, this systems has been validated and found to accurately predict the fermentation of fibers in human subjects by presenting the same magnitude of the differences in SCFA production [43]. The current study provides more evidence for the consumption of specific ingredients with the aim of modulating the gut microbiota in the context of obesity.

Impact of the substrates on metabolic activity

When compared against each other and against the control, both fibers presented different fermentation kinetics (in terms of SCFA production) (Fig 1). The difference between the proportion of propionate and $n$-butyrate in AG experiments using both microbiotas (Fig S2) is interesting in the light of the discussion of the impact of microbial metabolites on obesity. The increase in propionate production in the experiments with the obese microbiota suggests that via this metabolite AG could be protective against inflammation and promote satiety in obese subjects.

Despite that most studies about the anti-inflammatory role of SCFA have been focused on the effects of $n$-butyrate and acetate [47,48], there is evidence pointing to propionate as a metabolite with a strong role against inflammation [49]. On the one hand, propionate acts as a ligand of G-protein-couple receptors (GPCR) 41 and 43 [50]. These receptors when activated, induce an increase of GLP-1 (which slows down gastric emptying and promotes satiety) and PYY (which up-regulates food digestion and absorption). Besides this, it has also been shown that when they are absent (at least for GPCR43 in knockout mice) there is an exacerbated inflammation in inflammatory-disease models [51]. Moreover, propionate has been found to decrease fatty acid levels in plasma [47]. As there is also evidence indicating that high plasma levels of fatty acids cause inflammation and 
consequently insulin resistance, the postulation of propionate as a molecule with anti-obesity properties is reinforced.

On the other hand, propionate has also been linked to autism [52] and hence, an increase in this metabolite may not be desirable, although the mechanism is not entirely clear.

In this study, IN was found to increase the production of $n$-butyrate in the fermentations with the lean microbiota (Fig S2) when compared to the obese. The butyrogenic effect of IN has been previously observed in vivo and in vitro [16,18,53-57]. $n$-Butyrate has been postulated as a molecule with health benefits for the human host since it has been found to be an important source of energy for colonocytes, with a potential protective role against colon cancer. In addition, it has been inversely correlated with inflammatory bowel diseases such as Crohn's disease [58-61]. To our knowledge only two studies have tested in vitro the fermentation of IN in both microbiotas (lean and obese). Both Sarbini et al [16] and Bussolo de Souza et al [18] found that the obese fermentation of IN produced higher concentrations of $n$-butyrate when compared to the lean fermentation. The inocula composition is not the same all the time (Table S2) and that may have contributed to our results. Nevertheless, we have observed that there are some compositional similarities in both inocula despite being prepared in different years (Table S2).

The fermentation of AG showed decreased BCFA concentrations in the fermentations with the obese microbiota when compared the lean. This supports the work from Vince and colleagues [62] and Robinson \& Slavin [20] who observed a significant decreased in products from proteolytic fermentation (specifically ammonia) by intestinal bacteria after the supplementation of AG. However, our study brings evidence, for the first time, about this beneficial effect of AG in microbiota from obese subjects.

Though production of BCFA was not lower in the obese microbiota fermenting IN when compared to lean, at least, when it is compared to control, the fermentation of IN with the lean microbiota is lower, giving also a good indication of the prebiotic effect of IN in ameliorating proteolytic fermentation. This effect has been previously observed in vitro as well as in vivo $[29,63,64]$.

\section{Energy extraction}

The hypothesis that the gut microbiota in obese individuals facilitates the additional extraction of calories from diet has been previously reviewed $[38,65,66]$. In the current study we were not able to confirm that the energy yield (in terms of SCFA produced) was higher in the feces from the obese donors in comparison with the lean donors (Table S1). However, the absorption of SCFA has been described 
as a very efficient process since only $5-10 \%$ is excreted in the feces [67]. Furthermore, in a study performed by Jumpertz and colleagues [68] overfeeding in lean subjects was associated with a greater decrease in stool energy loss showing a relation between loss of energy in feces and energy load. Therefore, based on our results we could hypothesize that obese individuals may have higher capacity of absorbing SCFA in their gut and, therefore, may have lower amounts of these metabolites in their feces compared with lean subjects.

However, we found a substrate dependent effect on the metabolic activity and consequently energy extraction when fermentations with both AG and IN were performed. These findings confirm our previous observations where a higher amount of energy extracted from the fermentation of IN was also found after fermentation by obese microbiota when compared to lean [18]. Although the difference is small, over a prolonged period this may add up to several kilos of body weight, since, as previously mentioned, an elevated production of SCFA contributes to a higher energy input to the host. But at the same time these metabolites have also been found to present satiety-enhancing properties via the activation of GPCRs. Thus, it could be that the enhancement of the production of these metabolites could be protective against obesity in this population, despite their energy content. Therefore, more research is needed in this area.

\section{Compositional changes}

When the inocula prepared from both lean and obese subjects (previous study; 2012) is compared to the inocula from the present study (2014), we observed some compositional differences in the shared bacterial groups. This shows that not all inocula are the same. However, there are some similarities in some increased groups observed from the $\mathrm{L} / \mathrm{O}$ ratios (Table S2) making it possible to distinguish certain bacteria belonging for example to Gemmiger, Dorea, Roseburia, Alistipes genera which both times were highly abundant in the lean microbiota and deserve being more investigated about their potential role in leanness.

After fermenting AG by the different microbiotas the growth of some groups of bacteria was highly stimulated in one microbiota whilst they decreased in the other. Between the groups that were benefited from the fermentation of AG in the obese microbiota we found Faecalibacterium, Dorea and Blautia. Judging from the ratios calculated in order to compare both inocula (L/O 2014; Table S2), it seems that the community from obese donors tend to re-structure towards the microbiota from lean donors after the fermentation of AG (Table 2). This effect was also previously observed by Bussolo de Souza et al [18] and Condezo-Hoyos et al [19] when testing the prebiotic effects of cassava bagasse and different apple cultivars on the composition from lean and obese microbiota. 
Lactobacillus was observed to increase in the obese microbiota fermenting AG (Table 2). Furthermore, we also described an increase of the species L. mucosae and L.gasseri in the obese microbiota after fermenting AG. Our results are in agreement with Robinson \& Slavin [20] when showing that a diet supplemented with AG increased the concentration of Lactobacillus in the feces from healthy participants. In addition, a study conducted by Santacruz et al [69] showed a parallel reduction of body mass index (BMI) and increase of Lactobacillus spp. concentrations in obese adolescents suggesting a potential role of this species in obesity and body weight control. Based on these findings we suggest that our study brings more evidence about how a prebiotic such as AG can beneficially influence the composition of the microbiota from obese subjects in weight management.

The lean microbiota fermenting AG presented an increase in B. thetaiotaomicron. The growth of this species on AG has also been found by others [70]. $B$. thetaiotaomicron colonization has been observed to elicit gene expression involved with the fortification of the intestinal barrier function and the maintenance of mucosal integrity which may suggest to be specially beneficial in obese subjects [71].

The obese microbiota composition on IN shifted the simulated gut environment into a more healthy milieu with increase of beneficial bacteria belonging to the Faecalibacterium, Blautia, Fusicatenibacterium and Bifidobacterium genera. The bifidogenic effect of IN was demonstrated to be more pronounced in the obese microbiota when compared to lean (Table 3). Interestingly, this effect was shown to selectively stimulate the growth of B. adolescentis (Table S4). This is in agreement with the selective upregulation of $B$. adolescentis by IN also found by Ramirez-Farias et al [22] and the selective upregulation of B.animalis by Venema and Maathuis [72]. In fact the latter authors hypothesized that within the Bifidobacterium genus, the diversity was diminished by inulin. This was also observed to some degree in our experiments (Table S4).

The difference in the bifidogenic effect of IN in the obese microbiota, especially in the specific case of $B$. adolescentis growth, can be explained by i) a long-known inverse relation between initial amounts present and the observed increase [73]. As observed e.g. by Korpela et al [74] and other authors, the lower the abundance of Bifidobacterium spp. at the starting point of an intervention, the more the increase observed after the administration of an specific prebiotic, and vice versa [74-76]; or ii) the high specificity of IN for stimulating the growth of certain bifidobacterial species as observed by Venema \& Maathuis [72]. A high abundance of bifidobacteria could be protective in obesity since it is speculated that this bacteria may decrease pro-inflammatory cytokines and decrease endotoxaemia which can improve glucose-induced insulin and glucose tolerance [16,77]. 


\section{Conclusion}

First line strategies to combat obesity include exercise and/or a balanced dietary regime. Though apparently simple, such changes in people's life are difficult to maintain and in most of the cases subjects struggle to follow these recommendations. Due to the complexity of this condition, it is vital to identify weight loss methods by which subjects can successfully achieve long-term results. In this respect, using fibers is a potential tool to supplement diet in weight management due to their satiety aspects, as well as in modulating the gut microbiota. Here in this study we have identified the potential of arabinogalactan and inulin in stimulating a gut community more related to a lean profile. However, these effects should be studied further in humans focusing especially on the role of these fibers in satiety.

\section{Acknowledgments}

The authors thank Freddy J. Troost and Bouke Salden for facilitating the recruitment of the donors, and declare that there is no conflict of interest. 
Lean vs Obese: arabinogalactan, inulin fermentation

\section{References}

1. Yang J, Keshavarzian A, Rose DJ (2013) Impact of dietary fiber fermentation from cereal grains on metabolite production by the fecal microbiota from normal weight and obese individuals. J Med Food 16: 862-867.

2. Elli M, Colombo O, Tagliabue A (2010) A common core microbiota between obese individuals and their lean relatives? Evaluation of the predisposition to obesity on the basis of the fecal microflora profile. Med Hypotheses 75: 350-352.

3. Lozupone CA, Stombaugh JI, Gordon JI, Jansson JK, Knight R (2012) Diversity, stability and resilience of the human gut microbiota. Nature 489: 220-230.

4. Topping DL, Clifton PM (2001) Short-chain fatty acids and human colonic function: roles of resistant starch and nonstarch polysaccharides. Physiol Rev 81: 10311064.

5. Russell WR, Gratz SW, Duncan SH, Holtrop G, Ince J, et al. (2011) High-protein, reduced-carbohydrate weight-loss diets promote metabolite profiles likely to be detrimental to colonic health. Am J Clin Nutr 93: 1062-1072.

6. Freeland KR, Wolever TM (2010) Acute effects of intravenous and rectal acetate on glucagon-like peptide-1, peptide YY, ghrelin, adiponectin and tumour necrosis factor-alpha. Br J Nutr 103: 460-466.

7. Tarini J, Wolever TM (2010) The fermentable fibre inulin increases postprandial serum short-chain fatty acids and reduces free-fatty acids and ghrelin in healthy subjects. Appl Physiol Nutr Metab 35: 9-16.

8. Anderson JW, Baird P, Davis RH, Ferreri S, Knudtson M, et al. (2009) Health benefits of dietary fiber. Nutr Rev 67: 188-205.

9. Liu S, Willett WC, Manson JE, Hu FB, Rosner B, et al. (2003) Relation between changes in intakes of dietary fiber and grain products and changes in weight and development of obesity among middle-aged women. Am J Clin Nutr 78: 920-927.

10. Royall D, Wolever TM, Jeejeebhoy KN (1990) Clinical significance of colonic fermentation. Am J Gastroenterol 85: 1307-1312.

11. Turnbaugh PJ, Ley RE, Mahowald MA, Magrini V, Mardis ER, et al. (2006) An obesity-associated gut microbiome with increased capacity for energy harvest. Nature 444: 1027-1131.

12. Schwiertz A, Taras D, Schafer K, Beijer S, Bos NA, et al. (2010) Microbiota and SCFA in lean and overweight healthy subjects. Obesity (Silver Spring) 18: 190-195.

13. Turnbaugh PJ, Hamady M, Yatsunenko T, Cantarel BL, Duncan A, et al. (2009) A core gut microbiome in obese and lean twins. Nature 457: 480-484.

14. Aguirre M, Jonkers DM, Troost FJ, Roeselers G, Venema K (2014) In vitro characterization of the impact of different substrates on metabolite production, 
energy extraction and composition of gut microbiota from lean and obese subjects. PLoS One 9: e113864.

15. Payne AN, Chassard C, Zimmermann M, Muller P, Stinca S, et al. (2011) The metabolic activity of gut microbiota in obese children is increased compared with normal-weight children and exhibits more exhaustive substrate utilization. Nutr Diabetes 1: e12.

16. Sarbini SR, Kolida S, Gibson GR, Rastall RA (2013) In vitro fermentation of commercial alpha-gluco-oligosaccharide by faecal microbiota from lean and obese human subjects. Br J Nutr 109: 1980-1989.

17. Sarbini SR, Kolida S, Deaville ER, Gibson GR, Rastall RA (2014) Potential of novel dextran oligosaccharides as prebiotics for obesity management through in vitro experimentation. Br J Nutr 112: 1303-1314.

18. Bussolo de Souza C, Roeselers G, Troost F, Jonkers D, Koenen ME, et al. (2014) Prebiotic effects of cassava bagasse in TNO's in vitro model of the colon in lean versus obese microbiota. J Funct Foods 11: 210-220.

19. Condezo-Hoyos L, Mohanty IP, Noratto GD (2014) Assessing non-digestible compounds in apple cultivars and their potential as modulators of obese faecal microbiota in vitro. Food Chem 161: 208-215.

20. Robinson RR, Feirtag J, Slavin JL (2001) Effects of dietary arabinogalactan on gastrointestinal and blood parameters in healthy human subjects. $J$ Am Coll Nutr 20: 279-285.

21. Crociani F, Alessandrini A, Mucci MM, Biavati B (1994) Degradation of complex carbohydrates by Bifidobacterium spp. Int J Food Microbiol 24: 199-210.

22. Ramirez-Farias C, Slezak K, Fuller Z, Duncan A, Holtrop G, et al. (2009) Effect of inulin on the human gut microbiota: stimulation of Bifidobacterium adolescentis and Faecalibacterium prausnitzii. Br J Nutr 101: 541-550.

23. Daguet D, Pinheiro I, Verhelst A, Possemiers S, Marzorati M (2016) Arabinogalactan and fructooligosaccharides improve the gut barrier function in distinct areas of the colon in the Simulator of the Human Intestinal Microbial Ecosystem. J Funct Foods 20: 369-379.

24. Kim LS, Waters RF, Burkholder PM (2002) Immunological activity of larch arabinogalactan and Echinacea: a preliminary, randomized, double-blind, placebo-controlled trial. Altern Med Rev 7: 138-149.

25. Hauer J, Anderer FA (1993) Mechanism of stimulation of human natural killer cytotoxicity by arabinogalactan from Larix occidentalis. Cancer Immunol Immunother 36: 237-244.

26. Hotamisligil GS (2006) Inflammation and metabolic disorders. Nature 444: 860867.

27. Aguirre M, Ramiro-Garcia J, Koenen ME, Venema K (2014) To pool or not to pool? Impact of the use of individual and pooled fecal samples for in vitro fermentation studies. J Microbiol Methods 107C: 1-7. 
28. Aguirre M, Eck A, Koenen ME, Savelkoul PHM, Budding AE, et al. (2015) Evaluation of an optimal preparation of human standardized fecal inocula for in vitro fermentation studies. J Microbiol Methods 117: 78-84.

29. van Nuenen MH, Diederick Meyer P, Venema K (2003) The effect of various inulins and Clostridium difficile on the metabolic activity of the human colonic microbiota in vitro. Microb Ecol Health Dis 15: 137-144.

30. de Graaf AA, Maathuis A, de Waard P, Deutz NE, Dijkema C, et al. (2010) Profiling human gut bacterial metabolism and its kinetics using [U13C]glucose and NMR. NMR Biomed 23: 2-12.

31. Maathuis A, Hoffman A, Evans A, Sanders L, Venema K (2009) The effect of the undigested fraction of maize products on the activity and composition of the microbiota determined in a dynamic in vitro model of the human proximal large intestine. J Am Coll Nutr 28: 657-666.

32. Van der Kamp JW, Jones J, Mccleary B, Topping DJ (2010) Dietary Fibre: New Frontiers for Food and Health: Wageningen Academic Publishers.

33. Marino PL, Sutin KM (2012) The ICU Book: Wolters Kluwer Health, Philadelphia, USA.

34. DeSantis TZ, Hugenholtz P, Larsen N, Rojas M, Brodie EL, et al. (2006) Greengenes, a chimera-checked 16S rRNA gene database and workbench compatible with ARB. Appl Environ Microbiol 72: 5069-5072.

35. Cole JR, Wang Q, Fish JA, Chai B, McGarrell DM, et al. (2014) Ribosomal Database Project: data and tools for high throughput rRNA analysis. Nucleic Acids Res 42: D633-642.

36. Lyon MR, Kacinik V (2012) Is There a Place for Dietary Fiber Supplements in Weight Management? Curr Obes Rep 1: 59-67.

37. Spill MK, Birch LL, Roe LS, Rolls BJ (2011) Hiding vegetables to reduce energy density: an effective strategy to increase children's vegetable intake and reduce energy intake. Am J Clin Nutr 94: 735-741.

38. Aguirre M, Venema K (2015) Does the Gut Microbiota Contribute to Obesity? Going beyond the Gut Feeling. Microorganisms 3: 213-235.

39. Backhed F, Ding H, Wang T, Hooper LV, Koh GY, et al. (2004) The gut microbiota as an environmental factor that regulates fat storage. Proc Natl Acad Sci U S A 101: 15718-15723.

40. Turnbaugh PJ, Backhed F, Fulton L, Gordon JI (2008) Diet-induced obesity is linked to marked but reversible alterations in the mouse distal gut microbiome. Cell Host Microbe 3: 213-223.

41. Wanders AJ, Jonathan MC, van den Borne JJ, Mars M, Schols HA, et al. (2013) The effects of bulking, viscous and gel-forming dietary fibres on satiation. $\mathrm{Br}$ J Nutr 109: 1330-1337.

42. Cummings JH, Edmond LM, Magee EA (2004) Dietary carbohydrates and health: do we still need the fibre concept? Clin Nutr Suppl 1: 5-17. 
43. Daniel M, Wisker E, Rave G, Feldheim W (1997) Fermentation in human subjects of nonstarch polysaccharides in mixed diets, but not in a barley fiber concentrate, could be predicted by in vitro fermentation using human fecal inocula. J Nutr 127: 1981-1988.

44. Bach Knudsen KE, Wisker E, Daniel M, Feldheim W, Eggum BO (1994) Digestibility of energy, protein, fat and non-starch polysaccharides in mixed diets: comparative studies between man and the rat. Br J Nutr 71: 471-487.

45. Wisker E, Knudsen KEB, Daniel M, Eggum BrO, Feldheim W (1997) Energy Values of Non-Starch Polysaccharides: Comparative Studies in Humans and Rats. J Nutr 127: 108-116.

46. Cummings JH, Beatty ER, Kingman SM, Bingham SA, Englyst HN (1996) Digestion and physiological properties of resistant starch in the human large bowel. Br J Nutr 75: 733-747.

47. Al-Lahham SH, Peppelenbosch MP, Roelofsen H, Vonk RJ, Venema K (2010) Biological effects of propionic acid in humans; metabolism, potential applications and underlying mechanisms. Biochim Biophys Acta 1801: 11751183.

48. Roelofsen H, Priebe MG, Vonk RJ (2010) The interaction of short-chain fatty acids with adipose tissue: relevance for prevention of type 2 diabetes. Benef Microbes 1: 433-437.

49. Al-Lahham S, Roelofsen H, Rezaee F, Weening D, Hoek A, et al. (2012) Propionic acid affects immune status and metabolism in adipose tissue from overweight subjects. Eur J Clin Invest 42: 357-364.

50. Brown AJ, Goldsworthy SM, Barnes AA, Eilert MM, Tcheang L, et al. (2003) The Orphan G protein-coupled receptors GPR41 and GPR43 are activated by propionate and other short chain carboxylic acids. J Biol Chem 278: 1131211319.

51. Maslowski KM, Vieira AT, Ng A, Kranich J, Sierro F, et al. (2009) Regulation of inflammatory responses by gut microbiota and chemoattractant receptor GPR43. Nature 461: 1282-1286.

52. MacFabe DF, Cain NE, Boon F, Ossenkopp KP, Cain DP (2011) Effects of the enteric bacterial metabolic product propionic acid on object-directed behavior, social behavior, cognition, and neuroinflammation in adolescent rats: Relevance to autism spectrum disorder. Behav Brain Res 217: 47-54.

53. van de Wiele T, Boon N, Possemiers S, Jacobs H, Verstraete W (2007) Inulin-type fructans of longer degree of polymerization exert more pronounced in vitro prebiotic effects. J Appl Microbiol 102: 452-460.

54. Grootaert C, Van den Abbeele P, Marzorati M, Broekaert WF, Courtin CM, et al. (2009) Comparison of prebiotic effects of arabinoxylan oligosaccharides and inulin in a simulator of the human intestinal microbial ecosystem. FEMS Microbiol Ecol 69: 231-242. 
55. Hughes SA, Shewry PR, Li L, Gibson GR, Sanz ML, et al. (2007) In vitro fermentation by human fecal microflora of wheat arabinoxylans. J Agric Food Chem 55: 4589-4595.

56. Sakaguchi E, Sakoda C, Toramaru Y (1998) Caecal fermentation and energy accumulation in the rat fed on indigestible oligosaccharides. Br J Nutr 80: 469-476.

57. Juskiewicz J, Zdunczyk Z, Frejnagel S (2007) Caecal parameters of rats fed diets supplemented with inulin in exchange for sucrose. Arch Anim Nutr 61: 201210.

58. Sokol H, Pigneur B, Watterlot L, Lakhdari O, Bermudez-Humaran LG, et al. (2008) Faecalibacterium prausnitzii is an anti-inflammatory commensal bacterium identified by gut microbiota analysis of Crohn disease patients. Proc Natl Acad Sci U S A 105: 16731-16736.

59. Velazquez OC, Lederer HM, Rombeau JL (1997) Butyrate and the colonocyte. Production, absorption, metabolism, and therapeutic implications. Adv Exp Med Biol 427: 123-134.

60. Willing B, Halfvarson J, Dicksved J, Rosenquist M, Jarnerot G, et al. (2009) Twin studies reveal specific imbalances in the mucosa-associated microbiota of patients with ileal Crohn's disease. Inflamm Bowel Dis 15: 653-660.

61. Basson MD, Turowski GA, Rashid Z, Hong F, Madri JA (1996) Regulation of human colonic cell line proliferation and phenotype by sodium butyrate. Dig Dis Sci 41: 1989-1993.

62. Vince AJ, McNeil NI, Wager JD, Wrong OM (1990) The effect of lactulose, pectin, arabinogalactan and cellulose on the production of organic acids and metabolism of ammonia by intestinal bacteria in a faecal incubation system. $\mathrm{Br}$ J Nutr 63: 17-26.

63. De Preter V, Falony G, Windey K, Hamer HM, De Vuyst L, et al. (2010) The prebiotic, oligofructose-enriched inulin modulates the faecal metabolite profile: an in vitro analysis. Mol Nutr Food Res 54: 1791-1801.

64. De Preter V, Vanhoutte T, Huys G, Swings J, De Vuyst L, et al. (2007) Effects of Lactobacillus casei Shirota, Bifidobacterium breve, and oligofructose-enriched inulin on colonic nitrogen-protein metabolism in healthy humans. Am $J$ Physiol Gastrointest Liver Physiol 292: G358-368.

65. DiBaise JK, Zhang H, Crowell MD, Krajmalnik-Brown R, Decker GA, et al. (2008) Gut microbiota and its possible relationship with obesity. Mayo Clin Proc 83: 460-469.

66. Tilg H (2010) Obesity, metabolic syndrome, and microbiota: multiple interactions. J Clin Gastroenterol 44 Suppl 1: S16-18.

67. de Graaf AA, Venema K (2008) Gaining insight into microbial physiology in the large intestine: a special role for stable isotopes. Adv Microb Physiol 53: 73168. 
68. Jumpertz R, Le DS, Turnbaugh PJ, Trinidad C, Bogardus C, et al. (2011) Energybalance studies reveal associations between gut microbes, caloric load, and nutrient absorption in humans. Am J Clin Nutr 94: 58-65.

69. Santacruz A, Marcos A, Warnberg J, Marti A, Martin-Matillas M, et al. (2009) Interplay between weight loss and gut microbiota composition in overweight adolescents. Obesity (Silver Spring) 17: 1906-1915.

70. Macfarlane GT, Macfarlane S, Gibson GR (1995) Co-culture of Bifidobacterium adolescentis and Bacteroides thetaiotaomicron in arabinogalactan-limited chemostats: effects of dilution rate and pH. Anaerobe 1: 275-281.

71. Zocco MA, Ainora ME, Gasbarrini G, Gasbarrini A (2007) Bacteroides thetaiotaomicron in the gut: molecular aspects of their interaction. Dig Liver Dis 39: 707-712.

72. Venema K, Maathuis AJ (2003) A PCR-based method for identification of bifidobacteria from the human alimentary tract at the species level. FEMS Microbiol Lett 224: 143-149.

73. Roberfroid MB (2001) Prebiotics: preferential substrates for specific germs? Am J Clin Nutr 73: 406S-409S.

74. Korpela K, Flint HJ, Johnstone AM, Lappi J, Poutanen K, et al. (2014) Gut microbiota signatures predict host and microbiota responses to dietary interventions in obese individuals. PLoS One 9: e90702.

75. Davis LM, Martinez I, Walter J, Hutkins R (2010) A dose dependent impact of prebiotic galactooligosaccharides on the intestinal microbiota of healthy adults. Int J Food Microbiol 144: 285-292.

76. Kolida S, Meyer D, Gibson GR (2007) A double-blind placebo-controlled study to establish the bifidogenic dose of inulin in healthy humans. Eur J Clin Nutr 61: 1189-1195.

77. Cani PD, Neyrinck AM, Fava F, Knauf C, Burcelin RG, et al. (2007) Selective increases of bifidobacteria in gut microflora improve high-fat-diet-induced diabetes in mice through a mechanism associated with endotoxaemia. Diabetologia 50: 2374-2383. 


\section{Supplemental material}

Table S1. Metabolites and energy content (in terms of SCFA) measured in the feces from the volunteers.

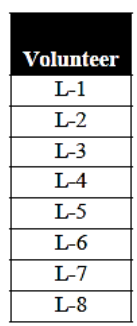

\begin{tabular}{|c|c|c|c|}
\hline \multicolumn{4}{|c|}{ SCFA (mmol) } \\
\hline acetate & propionate & $n$-butyrate & Total \\
\hline 233.3 & 74.9 & 49.50 & 377.7 \\
\hline 219.7 & 58.1 & 64.80 & 342.6 \\
\hline 232.3 & 44.6 & 36.40 & 313.3 \\
\hline 83.6 & 34.1 & 26.50 & 144.2 \\
\hline 85.9 & 15.9 & 18.70 & 120.6 \\
\hline 89.5 & 33.0 & 37.80 & 160.2 \\
\hline 168.3 & 66.2 & 58.70 & 293.2 \\
\hline 205.8 & 69.3 & 69.60 & 344.8 \\
\hline \multicolumn{4}{|c}{} \\
\hline
\end{tabular}

\begin{tabular}{|c|c|c|}
\hline \multicolumn{3}{|c|}{ BCFA (mmol) } \\
\hline$i$-butyrate & $i$-valerate & Total \\
\hline 11.4 & 16.3 & 27.7 \\
\hline 7.89 & 10.0 & 17.9 \\
\hline 8.00 & 11.1 & 19.1 \\
\hline 4.57 & 6.91 & 11.5 \\
\hline 3.84 & 6.05 & 9.89 \\
\hline 8.30 & 12.7 & 21.0 \\
\hline 9.60 & 14.2 & 23.8 \\
\hline 9.42 & 14.3 & 23.7 \\
\hline
\end{tabular}

\begin{tabular}{|c|c|c|c|}
\hline \multicolumn{4}{|c|}{ Energy extraction (KJ) } \\
\hline acetate & propionate & $n$-butyrate & Total \\
\hline 204.2 & 115.1 & 108.4 & 427.6 \\
\hline 192.0 & 89.1 & 139.7 & 423.4 \\
\hline 203.3 & 68.6 & 79.9 & 351.5 \\
\hline 73.2 & 52.3 & 58.2 & 183.7 \\
\hline 75.3 & 24.5 & 41.1 & 140.6 \\
\hline 78.2 & 50.6 & 82.8 & 211.7 \\
\hline 147.3 & 101.7 & 128.9 & 377.4 \\
\hline 179.9 & 106.7 & 152.7 & 438.9 \\
\hline
\end{tabular}

\begin{tabular}{|l|}
\hline O-1 \\
\hline O-2 \\
\hline O-3 \\
\hline O-4 \\
\hline O-5 \\
\hline O-6 \\
\hline O-7 \\
\hline
\end{tabular}

\begin{tabular}{|c|c|c|c|}
\hline 138.6 & 43.1 & 35.1 & 219.9 \\
\hline 176.8 & 92.1 & 54.2 & 323.0 \\
\hline 112.2 & 32.8 & 34.3 & 179.3 \\
\hline 83.0 & 22.7 & 20.4 & 126.1 \\
\hline 157.2 & 37.8 & 39.3 & 234.3 \\
\hline 180.9 & 44.4 & 40.6 & 265.9 \\
\hline 109.5 & 30.0 & 20.4 & 159.9 \\
\hline
\end{tabular}

\begin{tabular}{|c|c|c|}
\hline 4.17 & 6.11 & 10.3 \\
\hline 0.00 & 1.62 & 1.62 \\
\hline 2.4 & 2.87 & 5.27 \\
\hline 2.78 & 4.17 & 6.96 \\
\hline 5.63 & 9.31 & 14.9 \\
\hline 5.07 & 7.64 & 12.7 \\
\hline 1.73 & 2.19 & 3.92 \\
\hline
\end{tabular}

\begin{tabular}{|c|c|c|c|}
\hline 121.3 & 66.1 & 77.0 & 264.4 \\
\hline 154.4 & 141.4 & 118.8 & 414.6 \\
\hline 98.3 & 50.2 & 75.3 & 223.8 \\
\hline 72.8 & 34.9 & 44.8 & 152.3 \\
\hline 137.7 & 58.2 & 86.2 & 281.6 \\
\hline 158.2 & 68.2 & 89.1 & 315.5 \\
\hline 95.8 & 46.0 & 44.8 & 186.6 \\
\hline
\end{tabular}

Table S2. Ratio L/O. Relative ratio of bacterial genera different between lean and obese at t0 in TIM-2 in the current study and in a study by Bussolo de Souza et al [18] in 2012.

\begin{tabular}{|l|c|c|}
\cline { 2 - 3 } \multicolumn{1}{l|}{} & $\mathbf{2 0 1 4}$ & $\mathbf{2 0 1 2}$ \\
\hline Bifidobacterium & 2.33 & 1.05 \\
\hline Lactobacillus & 0.78 & 0.07 \\
\hline Enterococcus & 0.85 & 0.31 \\
\hline Lactococcus & 53.63 & 0.24 \\
\hline Shigella & 0.21 & 0.34 \\
\hline Streptococcus & 20.01 & 0.35 \\
\hline Faecalibacterium & 74.82 & 680.58 \\
\hline Bacteroides & 2.81 & 3.99 \\
\hline Weissella & 2.09 & 0.99 \\
\hline Collinsella & 0.76 & 6.60 \\
\hline Gemmiger & 23.77 & 11.87 \\
\hline Blautia & 2.93 & 58.96 \\
\hline Roseburia & 5.64 & 205.17 \\
\hline Dorea & 30.39 & 17.83 \\
\hline Coprobacillus & 1.12 & 7.92 \\
\hline unclassified Erysipelotrichaceae & 0.16 & 14.14 \\
\hline Parabacteroides & 0.55 & 19.13 \\
\hline Anaerostipes & 1.30 & 131.47 \\
\hline Alistipes & 3.91 & 59.01 \\
\hline
\end{tabular}


Table S3. Relative change of bacterial species after $72 \mathrm{~h}$ of fermentation experiments of arabinogalactan and inulin in TIM-2 using microbiota from lean and obese individuals.

\begin{tabular}{|l|}
\hline Species \\
\hline Clostridium butyricum \\
\hline Bifidobacterium adolescentis \\
\hline unclassified Clostridium \\
\hline unclassified Bifidobacterium \\
\hline Lactobacillus mucosae \\
\hline unclassified Lactobacillus \\
\hline Unclassified \\
\hline Bifidobacterium longum \\
\hline Enterococcus faecium \\
\hline unclassified Enterococcus \\
\hline Faecalibacterium prausnitzii \\
\hline unclassified Bacteroides \\
\hline unclassified Faecalibacterium \\
\hline Bacteroides vulgatus \\
\hline Bacteroides caccae \\
\hline unclassified Eubacterium \\
\hline unclassified Lactococcus \\
\hline Bacteroides thetaiotaomicron \\
\hline $\begin{array}{l}\text { Bifidobacterium } \\
\text { pseudocatenulatum }\end{array}$ \\
\hline Lactobacillus gasseri \\
\hline Enterococcus faecalis \\
\hline Lactococcus lactis \\
\hline Enterococcus gallinarum \\
\hline
\end{tabular}

\begin{tabular}{|c|c|c|c|}
$\begin{array}{c}\text { L- } \\
\text { Arabino }\end{array}$ & $\begin{array}{c}\text { O- } \\
\text { Arabino }\end{array}$ & L-Inulin & O-Inulin \\
\hline 0.40 & 0.24 & 0.19 & 0.30 \\
\hline 0.01 & & 0.66 & 4.68 \\
\hline 0.41 & 0.80 & 0.28 & 0.62 \\
\hline 0.17 & 0.07 & 0.76 & 1.90 \\
\hline 0.50 & 2.06 & 1.08 & \\
\hline 0.58 & 3.14 & 0.71 & \\
\hline 1.03 & 2.29 & 0.89 & 2.02 \\
\hline 2.00 & 0.21 & 0.15 & 0.04 \\
\hline 0.50 & 1.48 & 0.90 & 0.58 \\
\hline 0.58 & 2.08 & 1.11 & 0.73 \\
\hline 0.40 & & 1.20 & \\
\hline 1.12 & & 3.91 & 0.59 \\
\hline 0.33 & & 0.84 & \\
\hline 0.07 & & & \\
\hline 44.35 & & & \\
\hline 0.51 & & 6.39 & \\
\hline 0.00 & & & \\
\hline 6.80 & & & \\
\hline & & & \\
\hline & 0.03 & 0.19 & \\
\hline & & 5.78 & \\
\hline & & 0.94 & \\
\hline & & 0.81 & \\
\hline & & & \\
\hline
\end{tabular}

Table S4. Bifidobacterium species abundance from the fermentation of inulin.

In both cases (L and O-fermentations) B.adolescentis dominated the Bifido species after $72 \mathrm{~h}$. In lean microbiota a complete domination is observed while in the obese microbiota it is about 25 fold higher (whereas at the start it was only $\sim 8 \%$ ). 
Lean vs Obese: arabinogalactan, inulin fermentation

\begin{tabular}{|l|c|c|}
\hline \multirow{2}{*}{ Species } & \multicolumn{2}{c|}{$\begin{array}{c}\text { Abundance (\% from total } \\
\text { reads) }\end{array}$} \\
\cline { 2 - 3 } & L-Inulin-t0 & L-Inulin-t72 \\
\hline Bifidobacterium adolescentis & 5.3 & 3.6 \\
\hline Bifidobacterium animalis & 0.3 & 0 \\
\hline $\begin{array}{l}\text { Bifidobacterium } \\
\text { pseudocatenulatum }\end{array}$ & 0.2 & 0 \\
\hline
\end{tabular}

\begin{tabular}{|l|c|c|}
\hline \multirow{2}{*}{\multicolumn{1}{|c|}{ Species }} & \multicolumn{2}{c|}{$\begin{array}{c}\text { Abundance (\% from total } \\
\text { reads) }\end{array}$} \\
\cline { 2 - 3 } & O-Inulin-t0 & O-Inulin-t72 \\
\hline Bifidobacterium adolescentis & 0.4 & 24.8 \\
\hline Bifidobacterium longum & 4.6 & 0.2 \\
\hline $\begin{array}{l}\text { Bifidobacterium } \\
\text { pseudocatenulatum }\end{array}$ & 0.2 & 1 \\
\hline Bifidobacterium bifidum & 0 & 0.1 \\
\hline
\end{tabular}

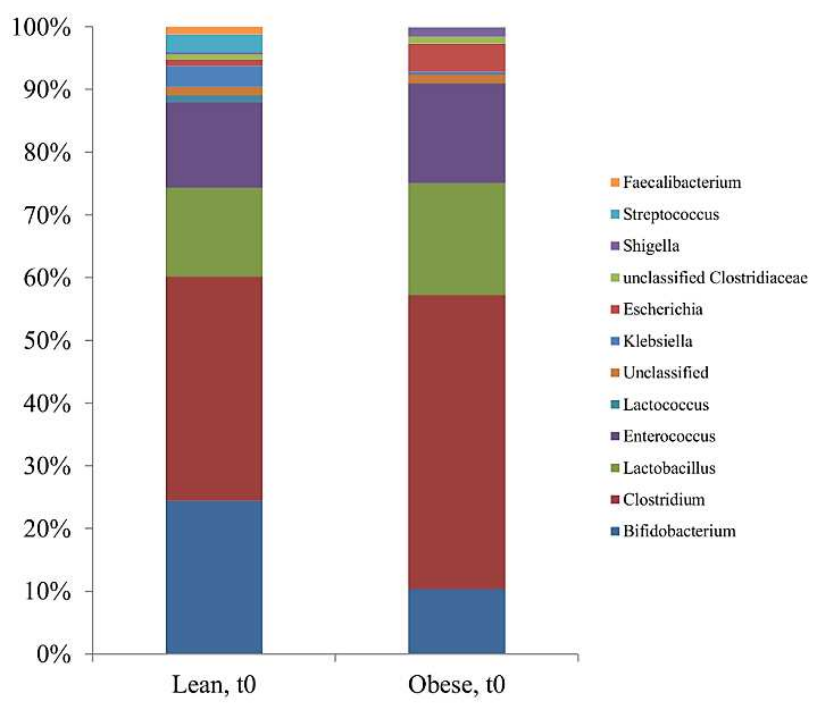

Fig. S1. Abundance $(\%)$ of the major groups of genera in the microbiota in feces of lean and obese subjects. 


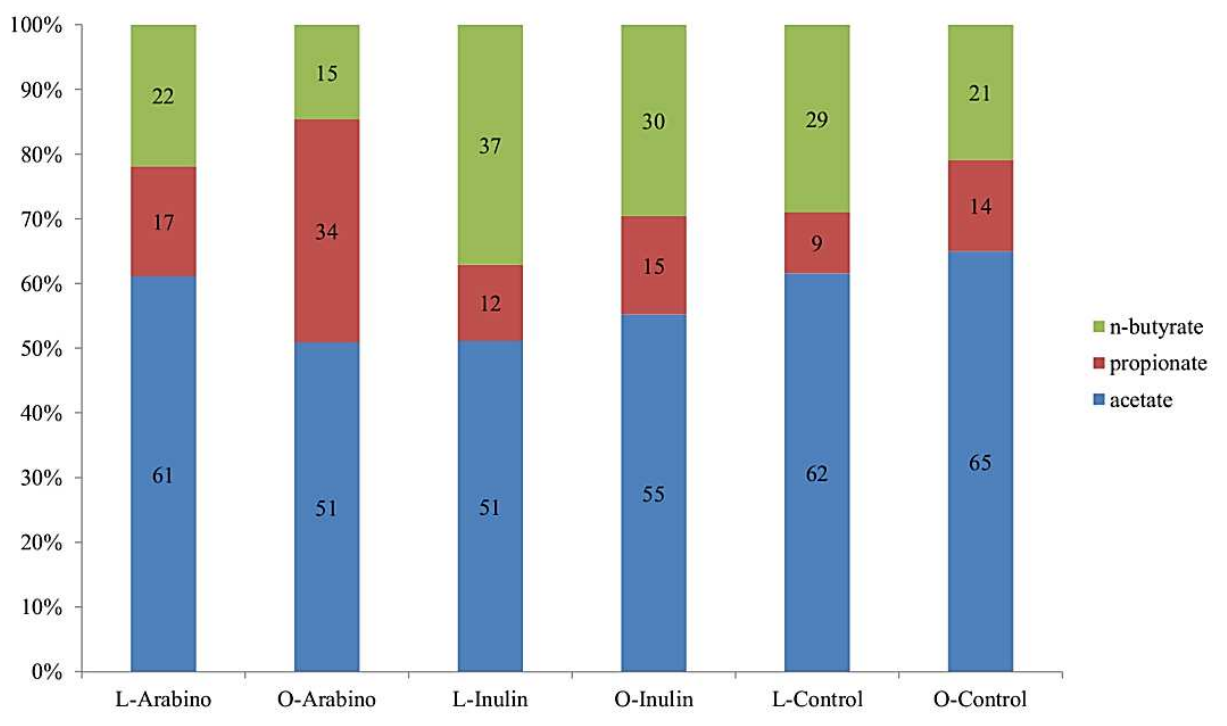

Fig. S2. SCFA ratios (\%) from the different diets at $\mathrm{t} 72$. 


\section{Chapter 8}

\section{Dynamics of defined and complex microbial communities fermenting mucin in an in vitro simulation of the human gut}




\section{Abstract}

The successful manipulation of the human gut bioreactor (also known as gut microbiota) requires to fully understand how it operates. This study is an attempt to evaluate the impact of mucin in the development of a defined (14 strains) and complex (lean and obese) fecal derived microbial communities colonizing an in vitro model of the large intestine (TIM-2 system). All communities were evaluated during $72 \mathrm{~h}$ fermentation of a diet containing mucin. The production of SCFA, BCFA and the extraction of energy (in terms of SCFA) was estimated. Furthermore, an array was used to determine the transcriptome of the bacteria before and after mucin addition.

Both metabolic and compositional data indicate that microbial communities present mucin specificity at a different degree. Complex communities were more efficient in metabolizing and extracting energy from mucin when compared to the defined community. The fermentation of mucin lead to a higher production of metabolites from proteolytic fermentation in all cases. Remarkably, the defined community presented the highest amount.

The addition of mucin facilitated the growth of species known by their mucolytic activity (e.g. Bifidobacterium bifidum, Lactobacillus mucosae, Bacteroides thetaiotaomicron). Furthermore, we also observed the growth of $F$. prausnitzii from mucin contributing to the scarce evidence currently available about the ability of this bacterium to metabolize (in vitro) this substrate.

The fermentation of mucin stimulated the expression of genes encoding enzymes involved in glycosaminoglycan degradation; valine, leucine and isoleucine biosynthesis; pyruvate metabolism; propanoate metabolism; butanoate metabolism; and pentose phosphate pathway. However, conclusions about this data need to be carefully done since due differences in the hybridization of the samples in the array were observed. Therefore, a normalization of the data needs to be performed. Overall, our study highlights the relevance of including mucin as a dietary substrate in an in vitro simulation of the human gut in order to increase the physiological relevance of the model as well as it sets a precedent for encouraging the exploration of distinctive health markers (metabolites and species). 
Defined vs Complex communities

\section{Introduction}

Due to the many roles in human health that the gut microbiota has been proposed to be involved in, there is growing interest in the identification and analysis of gut microbial communities in order to unfold specific characteristics from healthy and unhealthy individuals [1-3].

The human gut microbiota is dominated by two phyla: Bacteroidetes and the large phylum Firmicutes. However, Actinobacteria, Verrucomicrobia and Proteobacteria are also found in many people but in lower proportions [4]. Specific studies testing the effect of changes in diet on the gut microbiota evidence a major influence of nutrients on the composition and activity of this complex community. It has been proposed that the dominance in the composition of the human gut microbiota by the Bacteroidetes and Firmicutes phyla is an adaptive response to a continuous changing nutrient environment characteristic for the dynamic conditions in the human gut [5]. Despite this, our understanding of the diet-microbiota interactions is still in its infancy [5]. The particular response by different taxa in terms of utilization of specific nutrients and their interactions is not well understood.

Different models have been successfully used to follow up the effect of diet on the gut microbiota as a determinant in host health. In this regard, findings from studies using gnotobiotic mice are particularly interesting. Mouse models colonized with a defined community ranging from 2 to 12 bacterial species have been tremendously informative since the complexity of the gut microbiota is reduced and it has been shown that there is niche specialization and functional redundancy within members [5-8]. Some of these studies highlight the importance of host glycans for guaranteeing the stability in this ecosystem $[8,9]$. In this context, it would be tempting to suggest that the incorporation of a mucosal environment in a gut model may play an important role when studying the dynamics of the microbial community in response to different stimuli (e.g. prebiotic or probiotic consumption) due to the fact that mucin could increase the ecological significance of the model.

Physiologically relevant gut models are urgently needed to continue the effort of elucidating diet-microbiota interactions. Despite the substantial value of human studies as a golden model, these interventions are mostly limited to end-point measurements (fecal samples) which provide restricted information about mucosal microbes $[10,11]$. The latter also applies to in vitro models simulating the human gut. Although the integration of modules incorporating mucus is limited in in vitro models, some studies have included the use of microcosms covered in mucin (in SHIME [10]), mucin beads encased within a dialysis membrane [12] and mucin 
gels inserted in glass tubes [13] showing that via these models the dynamics between the mucosal and the luminal bacteria could be simulated. Moreover, in vitro models offer the great opportunity of studying microbe-microbe and microbesubstrate interactions in depth by carefully controlling all variables and avoiding host derived interactions which is, in this case, an advantage compared to animal and human models.

Here, we inoculated an in vitro system simulating the proximal colon (TIM-2; [14]) with i) a defined community harboring 14-members which represents the 5 most dominant phyla in the healthy human gut, ii) a pooled microbiota prepared from the feces from obese, or iii) lean volunteers.

The selection of the members of this defined consortium was based on phylogenetic diversity. Furthermore, these strains represent important gut commensals and have been identified as fermenters of amino acids and undigestible carbohydrates [6], shown to influence gut physiology [9,15-19], provide energy to the colonocytes [20,21], encode ABC-transporter associated substrate-binding proteins [22] and protect against pathogenic bacteria [15,23-25]. Interestingly, some of these species have been used as indicators of gut health since they are presumed to play a role in many disorders including cancer $[16,26]$, obesity [16], irritable bowel syndrome [16], inflammatory bowel diseases [16,27] and glucose intolerance [28].

The aim of this study was to gain insight in how the presence of mucin plays a role in the development of bacteria in an in vitro model closely simulating the human gut. For this reason, fermentation experiments using three different types of communities (i.e. defined community, and lean and obese fecal microbiota) were performed in the presence or absence of mucin in order to compare metabolic fermentation profiles as well as changes in the composition and gene expression during the fermentation experiments.

\section{Materials and methods}

\section{Bacterial strains and culture conditions}

Anaerobic culture methods were used for cultivation of the consortium. Table S1 shows the information about the strains used, which were obtained from public strain repositories (ATCC, Manassas, VA, USA or DSMZ, Braunschwei, Germany) and kept as frozen stocks with glycerol at $-80{ }^{\circ} \mathrm{C}$. Strains from frozen stocks were grown as monocultures to early $\log$ phase at $37^{\circ} \mathrm{C}$ (atmosphere $80 \%$ $\mathrm{N}_{2}, 10 \% \mathrm{CO}_{2}$ and $10 \% \mathrm{H}_{2}$; Whitley A45 anaerobic workstation, West Yorkshire, England) in RCM, TS, M17, MRS or Chopped Meat Medium for 2 to 3 days in 15 $\mathrm{ml}$ tubes. After autoclaving, different media were supplemented with filter 
sterilized: glucose, hemin and vitamin K (RCM and Chopped Meat Medium), just glucose (M17) or cysteine (added as a redox buffer; RCM, MRS and Chopped Meat Medium). For the specific case of $F$. prausnitzii, the strain was grown in YCFAG medium as described by Lopez-Siles et al. [29] and Duncan [30].

The cultures were collected at different $\mathrm{OD}_{600}$ values prior the pooling and inoculation in the in vitro system in an effort to ensure that none of the species had an early advantage during establishment and that $E$. coli would not overgrow the community as previously experienced in a pilot study (not shown).

\section{Complex microbiota}

The human fecal inocula used for the TIM-2 experiments consisted of an active, pooled fecal microbiota prepared from: i) 8 healthy lean volunteers (male: $n=4$, female: $\mathrm{n}=4$, average age $=31$ y (range: $25-42$ ), $\left.\mathrm{BMI}=20 \pm 1.48 \mathrm{~kg} / \mathrm{m}^{2}\right)$; ii) 7 healthy obese volunteers (male: $n=3$, female: $n=4$, average age $=51$ y (range: 29 $68)$, BMI $=32 \pm 1.17 \mathrm{~kg} / \mathrm{m}^{2}$ ). The exclusion criteria for lean and obese volunteers included the use of antibiotics during the preceding 3 months, gastrointestinal disease, severe chronic disease or food allergy and intake of probiotics and prebiotics. We have previously shown that pooling does not result in an aberrant microbiota composition or activity [31].

Fecal samples were collected using a container kit which was maintained under anaerobiosis by using anaerobic packs (AnaeroGen ${ }^{\mathrm{TM}}$, Oxoid, Cambridge, UK). Samples were homogenized in an anaerobic cabinet $\left(80 \% \mathrm{~N}_{2}, 10 \% \mathrm{CO}_{2}, 10 \% \mathrm{H}_{2}\right)$ as described by Aguirre et al. [32]. The resulting slurry was aliquoted and snapfrozen in liquid nitrogen $\left(-196^{\circ} \mathrm{C}\right)$. This microbiota was stored at $-80^{\circ} \mathrm{C}$ before inoculation in TIM-2.

\section{Gut fermentation experiments}

The TIM-2 system [14] was flushed with $\mathrm{N}_{2}$ for $9 \mathrm{~h}$ prior to the introduction of the inoculum and it was maintained under this condition at $37^{\circ} \mathrm{C}$ for $96 \mathrm{~h}$ with the $\mathrm{pH}$ kept at or above 5.8 by automatic titration with $2 \mathrm{M} \mathrm{NaOH}$. A $30 \mathrm{ml}$ portion of culture homogenate was used to inoculate the units for each experiment. The microbiota was left to adapt $(16 \mathrm{~h})$ to the new environment after inoculation and during this period the basal medium preparation (SIEM) was gradually introduced into the system in a total volume of $40 \mathrm{ml}$. Subsequently, a volume of $180 \mathrm{ml}$ of the different diets and control was administrated over the $72 \mathrm{~h}$ of the test period at a rate of $2.5 \mathrm{ml} / \mathrm{h}$.

In order to remove water and fermentation products from the lumen, a dialysate system, consisting of a semi-permeable hollow membrane (described in detail by 
van Nuenen et al.[33]), ran through the lumen. For all the experiments, the speed of the dialysis fluid was set at $1.5 \mathrm{ml} / \mathrm{min}$.

After 24 and $48 \mathrm{~h}$ of fermentation $25 \mathrm{ml}$ of lumen sample was removed from the system to mimic the transit of material coming from the proximal and reaching the distal colon [34]. Luminal and dialysate samples were taken after $\mathrm{t}=0,24,48$ and $72 \mathrm{~h}$. In all cases samples were snap-frozen in liquid nitrogen and stored $\left(-80{ }^{\circ} \mathrm{C}\right)$ until analysis (short-chain -SCFA- and branched-chain -BCFA- fatty acids performed in both luminal and dialysate samples from t0, 24, 48 and 72; composition and gene expression performed in luminal samples at t0 and t72).

The experiments were performed in duplicate per tested compound $(n=2)$. To avoid unnecessary repetition, this is not indicated further in the text or graphs in the results section. Results are displayed as average of these duplicates. In order to refer to the fermentation experiments using the inoculum from lean (L), obese (O) subjects or defined community (DC), in the following sections the letters $\mathrm{L}, \mathrm{O}$ or DC are tagged with -muc (for mucin) or -control (e.g. L-muc, O-muc, DC-muc).

\section{Fermentation media}

During the adaptation period ( $16 \mathrm{~h}$ ) all TIM-2 units were fed with simulated ileal efflux medium (SIEM) as described by Maathuis et al. [34]. After this adaptation period the units were fed with the same medium, without the $7.5 \mathrm{~g}$ standard carbohydrates, which were replaced by approximately $7.5 \mathrm{~g}$ of mucin from porcine stomach (Type II, Sigma) which constituted $12 \% \mathrm{w} \mathrm{w}^{-1}$ of the total medium. SIEM was used as a control.

Analysis of SCFA (acetate, propionate, and $n$-butyrate) and BCFA (iso-butyrate and iso-valerate)

Samples from the complex communities were prepared and analyzed as described previously [34]. Briefly, samples were centrifuged (12000 rpm at $4{ }^{\circ} \mathrm{C}$ for $10 \mathrm{~min}$ ). To the clear supernatant a mixture of formic acid (20\%), methanol and 2-ethyl butyric acid (internal standard, $2 \mathrm{mg} / \mathrm{ml}$ in methanol) was added. A $3 \mu \mathrm{l}$ sample with a split ratio of 75.0 was injected on a GC-column (ZB-5HT inferno, ID 0.52 $\mathrm{mm}$, film thickness $0.10 \mathrm{um}$; Zebron; Phenomenex, USA) in a Shimadzu GC-2014 gas chromatograph. Standard curves were obtained by injecting calibrated quantities of a blend of volatile fatty acids and amounts were calculated from the graph obtained correlating peak height and time measured (all reagents from Sigma-Aldrich with the exception of formic acid which was from Merck). Samples from the DC-experiments were analyzed as described by Ladirat et al. [35]. 
Defined vs Complex communities

\section{Energy extraction}

Energy extraction in the form of SCFA was calculated using the following Kcal $\mathrm{mol}^{-1}$ values for acetate, propionate and $n$-butyrate respectively: 209, 367 and 524 $[36,37]$.

\section{Characterization of bacterial population}

RNA was isolated from luminal samples using standard molecular biology kits from Invitrogen (ChargeSwitch ${ }^{\circledR}$ total RNA cell kit, CA, US) with some modifications.

RNA quantity and quality were assessed spectrophotometrically (ND-1000, NanoDrop Technologies, Wilmington, USA). Furthermore, gel electrophoresis was performed to study integrity of the RNA.

Reverse-transcriptase amplification of the 16S rRNA gene (V3-V4), barcoding and library preparation (1st step PCR and 2nd PCR) were performed by BaseClear, Leiden, The Netherlands.

Short paired-end sequence reads were generated using the Illumina MiSeq system and converted into FASTQ files using the BCL2FASTQ pipeline version 1.8.3. Quality trimming was applied based on Phred quality scores. Subsequently, the Illumina paired reads were merged into single reads (so-called pseudoreads) through sequence overlap (16S rRNA V3V4 region of about 500bp). Chimeric pseudoreads were removed and the remaining reads were aligned to a combination of the GreenGenes and RDP 16S gene databases [38,39]. Based on the alignment scores of the pseudoreads, the taxonomic classes were assigned by associating each pseudoread to the best matching Operational Taxonomic Unit (OTU). The taxonomic depth of the lineage was based on the identity threshold of the rank; Species 99\%, Genus 97\%, Family 95\%, Order 90\%, Class 85\%, Phylum 80\%.

Fold changes were calculated in order to discriminate the differential groups that were affected after the intervention when compared to control. The calculation was based on the ratio between a sampling time point and t0 (e.g., t72/t0). Then the ratio for this value and the control was determined to obtain fold changes compared to the control. A value within the range 0.95-1.05 indicates no change; a value of $>1.05$ indicates an increase; and a value of $<0.95$ indicates a decrease of the respective microbial genera/species (depicted in the manuscript as $\rightarrow$, $\uparrow$ and $\downarrow$, respectively) .

\section{Microarray procedures}

An RNA-based multi-species array was designed using a SurePrint ${ }^{\mathrm{TM}}$ G3 Custom Gene Expression Microarray (one glass slide formatted with four high-definition $180 \mathrm{~K}$ arrays; 4x180K; Agilent Technologies, US). This microarray contained 
175.000 probe sets and covered the complete genomes of the members of the DC and it allowed the analysis of the different transcription profiles. In silico, the full genome of each of the 14 strains was chopped in pieces of 500 base pairs for the preparation of the array. Then all those fragments were studied in silico and fragments that were in more than one species were discarded as a suitable probe. DNA fragments that were unique to one of the 14 strains were used as a probe target. Since all the homologous parts from genes were removed as described above, very homologous genes are underrepresented in this data set compared to the ones that are more divergent.

The obtained RNA from TIM-2 samples was transcribed into cDNA and then subjected to microarray hybridization. Targets for a one-color microarray experiment were generated. Cy3-labeled samples were produced and hybridized following the manufacturer's protocol (Stratagene Fairplay III Microarray Labeling Kit; Agilent Technologies). The hybridization procedure was performed according to the Agilent 60-mer oligo microarray processing protocol using the Agilent Gene Expression Hybridization Kit (Agilent Technologies). Subsequently, 600 ng Cy3labeled fragmented cDNA in hybridization buffer was hybridized $17 \mathrm{~h}$ at $65{ }^{\circ} \mathrm{C}$ to the microarray using Agilent's recommended hybridization chamber and oven. Finally, the microarrays were washed once with the Agilent Gene Expression wash buffer 1 for $1 \mathrm{~min}$ at room temperature followed by a second wash with preheated Agilent Gene Expression wash buffer $2\left(37^{\circ} \mathrm{C}\right)$ for $1 \mathrm{~min}$. The last washing step was performed in the presence of acetonitrile. Scanning of the fluorescence signals of the hybridized Agilent microarrays was performed with $5 \mu \mathrm{m}$ resolution using Agilent's Microarray Scanner System (Agilent Technologies, Palo Alto, USA) with XDR extended range. The Agilent Feature Extraction Software (v11.5) was used to obtain the microarray data.

Data were collected by using microarrays spots whose intensities were reproducibly higher than the background level. All the expression values (Expr) that were in the dynamic range were background subtracted and averaged for each array yielding an array-specific normalization factor $(\mathrm{N})$ that was used for calculating a normalized gene-expression values (Nexpr) per array. At the end, the set of data was $2 \log$ transformed.

Normalized expression was made per array as specified in equation 1:

Where:

$$
\text { Nexpr }=\left[(\text { Expr-avgBKGR }) / \mathrm{N}^{*} \operatorname{avgN} \mathrm{N}^{\text {AllArrays }}\right]+\operatorname{avgBKGR} \mathrm{R}^{\text {AllArray }}
$$

Expr $=$ raw expression value per gene per array (including background).

Nexpr $=$ normalized expression per gene of each array 
$\mathrm{N}=$ average (signal-background) per array, filtered for probes with significant \& not saturated signals (Expr $>2 * a v g B K G R ~ \& ~ E x p r<50000)$.

avgBKGR = average background per analyzed array

avgN ${ }^{\text {AllArrays }}=$ average normalization factor for all the arrays.

$\operatorname{avgBKGR}^{\text {AllArray }}=$ average standard background of all arrays. This background value reduced noise in expression values around background level, leading to false differentials.

A bias control was performed in order to find if the arrays presented certain grouping, beyond the bias expected for the tested conditions. Hierarchical clustering, correlation and PCA analyses were performed (TIGR MeV, MA, US).

Comparative measurements of transcript abundance was performed on time-course samples by directly comparing the abundance of each gene's transcripts relative to the t0 sample $(\mathrm{R}=\mathrm{Nexpr}[\mathrm{t}=72 \mathrm{~h}] / \mathrm{Nexpr}[\mathrm{t}=0 \mathrm{~h}])$. All probes were split in nonresponsive $(\mathrm{Abs}[2 \log \mathrm{R}]<2)$ from those that were responsive $(\mathrm{Abs}[2 \log R]>2)$. Over 175.000 probes, 22.000 showed significant differential expression. We used t-test $(\mathrm{P}<0.01)$ to select the most significant differentially expressed genes (DEGs) for either of 3 comparisons (Muc-Control at $72 \mathrm{~h}$ for DC, obese or lean fecal groups). Comparisons were made in time $(\mathrm{t}=72 \mathrm{~h} v s \mathrm{t}=0 \mathrm{~h})$ or between treatments (e.g. mucin $v s$ control at $\mathrm{t}=72 \mathrm{~h}$ )

A heat map was made of the data from the genes differentially expressed in each experiment (Mucin DC, Obese, Lean; t72) and that were present in the metabolic gene sets derived from the Kyoto Encyclopedia and Genes and Genomes (KEGG).

Quantitative PCR of selected expressed genes

To test the validity of the microarray results, we performed reverse transcriptionquantitative PCR (RT-q-PCR) to compare the abundance of Amu_0666, BAD_0688 and BT_0618 from mucin-treated and untreated communities. As internal control we analyzed the relative abundance of $16 \mathrm{~S}$ gene. The three genes were selected because they were differentially expressed with respect to their own control (i.e., Amuc_0666 was differentially expressed in DC; BAD_0688 in lean and obese; BT_0618 in lean and DC).

The primers used in the amplifications are listed in Table 1. All primers were designed by using Primer Express ${ }^{\circledR} 2.0$ software (Applied Biosystems, US). The $\mathrm{GC}$ content of the primers was in the range of 48 to $60 \%$ and $\mathrm{T}_{\mathrm{m}}$ from 79 to $84{ }^{\circ} \mathrm{C}$. 
Table 1. Primer sequences of target and reference genes used for RT-q-PCR.

\begin{tabular}{|c|l|c|c|c|}
\hline Name & \multicolumn{1}{|c|}{ Definition } & Forward primer (5'-3') & Reverse primer (5'-3') & $\left.\mathbf{T a}^{*} \mathbf{(}^{\mathbf{}} \mathbf{C}\right)$ \\
\hline Amuc_0666 & $\begin{array}{l}\text { C5-Branched dibasic } \\
\text { acid metabolism }\end{array}$ & GAACGCACCTTTGCCACTGT & CGGTTCCGCCTGGTTGT & 61 \\
\hline BAD_0688 & $\begin{array}{l}\text { phosphate } \\
\text { acetyltransferase }\end{array}$ & GCA GGCGCGCAAACA & TGCCCATCACCACAAGCAT & 62 \\
\hline $\begin{array}{l}\text { Nat-transporting } \\
\text { NADH:ubiquinone } \\
\text { oxidoreductase } \\
\text { electron transport } \\
\text { complex protein } \\
\text { RnfC }\end{array}$ & GTCGATGCGAGCGGTTATG & CCCATTCGTCGCCTTCTACA & 58 \\
\hline
\end{tabular}

$* \mathrm{Ta}=$ temperature of annealing

RT-q-PCR was performed for gene expression in a 7500 Real-Time PCR system (Applied Biosystem, US) with Relative Quantification Software (Applied Biosystem V.1.4) with fluorescence signal detection after each amplification cycle. Probes used for the targeted genes (Table 1) were labeled with VIC, NED or Cy5, and for the control gene (16S) with FAM.

Each reaction was performed in a $26 \mu \mathrm{l}$ volume containing $12.5 \mu \mathrm{l}$ of $1 \mathrm{X}$ QuantiTec ${ }^{\circledR}$ Multiplex PCR Mix (Qiagen), $5 \mu$ l RNA template, $1.0 \mu 1$ of reverse primer at $0.4 \mu \mathrm{M}, 0.5 \mu \mathrm{l}$ of forward primer at $0.2 \mu \mathrm{M}, 0.5 \mu \mathrm{l}$ of fluorophore at 0.1 $\mu \mathrm{M}$ and $0.25 \mu \mathrm{l}$ reverse transcriptase enzyme. Negative controls (with no RNA template) for each primer set were included in each run. To assess the contribution of contaminating DNA templates in the RNA samples, RNA controls without the RT-step were performed in parallel. Serial dilutions of DNA isolated from single strains were performed to generate a standard curve for each primer-probe combination in order to define the efficiency of the RT-q-PCR reaction. The thermal cycle conditions included reverse transcriptase reaction $\left(50{ }^{\circ} \mathrm{C}\right.$ for $\left.20 \mathrm{~min}\right)$, HotStarTaq DNA Polymerase activation $\left(95{ }^{\circ} \mathrm{C}\right.$ for $15 \mathrm{~min}$ ), followed by 2-step program for amplification and quantification of 45 cycles $\left(15 \mathrm{~s}\right.$ at $95{ }^{\circ} \mathrm{C}, 60{ }^{\circ} \mathrm{C}$ for 1 min with fluorescence measurement).

\section{Ethics Statement}

Studies using fecal donations from healthy volunteers do not require medical ethical committee approval in The Netherlands since they are considered as noninvasive. However, volunteers that donated a sample were informed prior to initiating the study and their participation was considered after providing a signed informed consent. The obese donors were recruited at Maastricht University Medical Center+ (The Netherlands). The lean donors were recruited at TNO (The Netherlands). The origins of the fecal donations were blinded using a code whose 
identity was known only by the responsible scientist. All results in the present study are referred to the pooled fecal inoculum and do not directly refer to a particular person.

\section{Results}

\section{Metabolic activity}

In the fermentation experiments with the complex microbiota mucin led to as high SCFA production as the control medium, SIEM. This was also the case for the DC, however, with the DC the production of SCFA was decreased notably in both control and the mucin intervention compared to the complex microbiotas (Fig. 1). The fermentation of mucin with the lean microbiota produced a higher amount of total SCFA (152.86 $\pm 1.30 \mathrm{mmol})$ when compared to the fermentation with the obese microbiota and DC $(142.01 \pm 3.60 \mathrm{mmol}$ and $112.11 \pm 15.09 \mathrm{mmol}$, respectively).

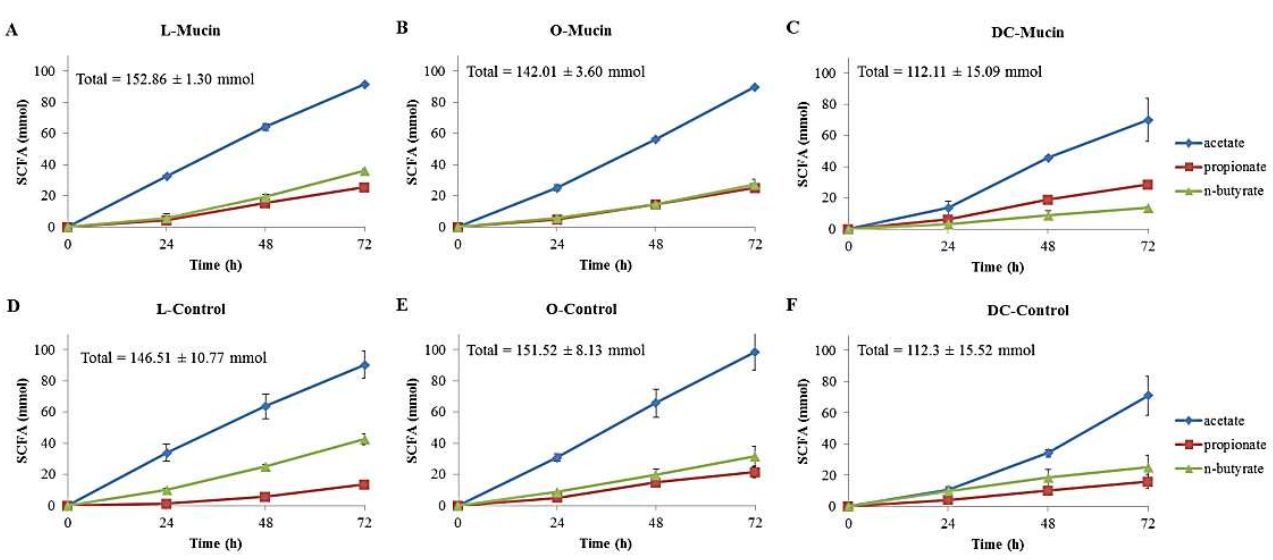

Fig. 1. Cumulative production of SCFA (mmol) during the $72 \mathrm{~h}$ fermentation.

Fermentation kinetics of mucin with both complex microbiotas was very similar. There was a clear dominance of acetate production through time, while propionate and $n$-butyrate values resembled each other quite closely throughout the fermentation. Kinetics of fermentation with the DC were different when compared to the complex microbiota. Production started slow. The production of propionate through time was higher than $n$-butyrate. Still, acetate was the metabolite produced in highest quantities (Fig. 1). Fermentation of the control medium (SIEM) showed similar characteristics as that of mucin, with butyrate higher than propionate with all three communities, with highest proportions for the lean microbiota. 


\section{$B C F A$}

The fermentation of mucin with the lean microbiota and DC stimulated the production of BCFA to a higher degree than the obese microbiota (Table 2). Values of BCFA in control experiments remained lower when compared to the respective mucin interventions. However, it is worth noticing that the DC experiments presented the highest cumulative production of BCFA when compared to the other two communities, also already in the control experiments.

\begin{tabular}{|c|c|c|c|c|c|c|c|c|c|}
\hline \multirow[b]{3}{*}{ Mucin } & \multicolumn{3}{|c|}{ Lean } & \multicolumn{3}{|c|}{ Obese } & \multicolumn{3}{|c|}{ Defined community } \\
\hline & $i$-butyrate & $i$-valerate & Total & $i$-butyrate & $i$-valerate & Total & $i$-butyrate & $i$-valerate & Total \\
\hline & $4.04 \pm 1.26$ & $7.20 \pm 0.09$ & $11.25 \pm 1.16$ & $2.11 \pm 0.02$ & $3.65 \pm 0.62$ & $5.77 \pm 0.64$ & $12.81 \pm 0.12$ & $12.67 \pm 0.39$ & $25.48 \pm 0.51$ \\
\hline Control & $0.74 \pm 0.14$ & $1.99 \pm 0.40$ & $2.73 \pm 0.27$ & $0.44 \pm 0.28$ & $1.22 \pm 0.06$ & $1.66 \pm 0.22$ & $6.45 \pm 0.43$ & $7.94 \pm 2.13$ & $14.38 \pm 2.57$ \\
\hline
\end{tabular}

\section{Energy extraction}

Lean microbiota extracted more energy $(47.3 \mathrm{Kcal}$; in the form of SCFA) from the fermentation of mucin when compared to the obese microbiota $(42.2 \mathrm{Kcal})$ or the DC (32.2 Kcal), in line with the higher production of SCFA (Fig. 2). The DC community grown on mucin extracted more energy from propionate when compared to the other microbiotas. Controls from the complex community were quite similar, whereas the DC-Control extracted less energy.

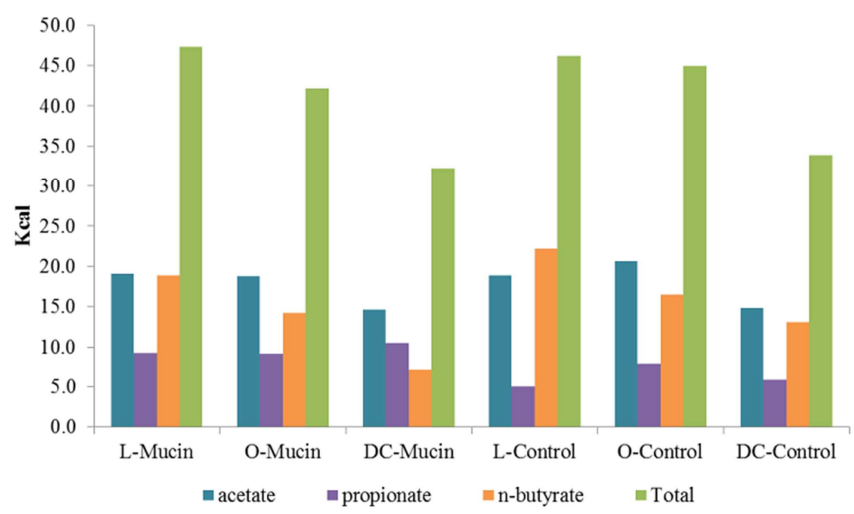

Fig. 2. Energy extraction. Values obtained after $72 \mathrm{~h}$ fermentation of mucin and control using microbiota from lean or obese subjects, or a defined community.

\section{Compositional changes}

\section{Genus level}

At the genus level, the majority of the bacteria responding to the fermentation of mucin in the DC experiments were also found in the L- and O- complex community (except 2 groups: Lachnoclostridium and Staphylococcus) (Table 3). 202 
Similarities in how the growth of these common groups was affected after the fermentation experiments (increase/decrease either in the 3 microbiotas or in 2) were observed, but also differences were found. The fold changes observed were at a different order of magnitude. For instance, the growth of Bifidobacterium in the DC community was highly stimulated upon the addition of mucin (12.2 fold change with respect to control), the increase in this group of bacteria was also observed in L-Muc, though not to the same degree (1.74 fold change with respect to control) while it decreased in O-Muc ( 0.17 fold change -read as $1 / 0.17=5.88$ fold decrease- with respect to control). With respect to Lactobacillus, it seemed that the addition of mucin to the lean microbiota highly benefited this group of bacteria when compared to the obese and the defined communities $(6.80,2.91$ and 1.10 fold change with respect to control, respectively).

Table 3. Relative change of shared bacterial genera after $72 \mathrm{~h}$ fermentation of mucin in TIM-2 with the defined and complex community. $\rightarrow$ indicates no change; $\uparrow$ indicates an increase; and $\downarrow$ indicates a decrease with respect to control. \% Values correspond to the relative abundance of the bacterial group in the mixtures at $\mathrm{t} 72$. Cells with no value correspond to bacteria that were not detected.

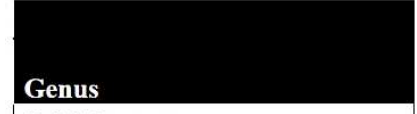

Bifidobacterium

Clostridium

Lactobacillus

Enterococcus

Unclassified

Klebsiella

Escherichia

unclassified Bifidobacteriaceae

unclassified Clostridiaceae

unclassified Lactobacillaceae

unclassified Enterococcaceae

Shigella

Bacteroides

unclassified Enterobacteriaceae

unclassified Streptococcaceae

unclassified Ruminococcaceae

Blautia

unclassified Bacteroidaceae unclassified Lachnospiraceae

Lachnoclostridium

Staphylococcus

\begin{tabular}{|c|c|}
\hline \multicolumn{2}{|c|}{ L-Muc-t72 } \\
\hline $\begin{array}{c}\text { Fold } \\
\text { change }\end{array}$ & $\begin{array}{c}\text { Abundance } \\
\text { (\%) }\end{array}$ \\
\hline$\uparrow$ & 21.54 \\
\hline$\uparrow$ & 4.86 \\
\hline$\uparrow$ & 2.53 \\
\hline$\downarrow$ & 4.21 \\
\hline$\uparrow$ & 2.56 \\
\hline$\uparrow$ & 0.29 \\
\hline$\uparrow$ & 0.30 \\
\hline$\rightarrow$ & 0.43 \\
\hline$\uparrow$ & 0.24 \\
\hline$\uparrow$ & 0.06 \\
\hline$\downarrow$ & 0.09 \\
\hline$\uparrow$ & 0.10 \\
\hline$\downarrow$ & 9.32 \\
\hline$\uparrow$ & 0.03 \\
\hline$\downarrow$ & 0.01 \\
\hline$\uparrow$ & 3.17 \\
\hline$\downarrow$ & 6.38 \\
\hline$\downarrow$ & 0.32 \\
\hline$\downarrow$ & 0.92 \\
\hline & \\
\hline & \\
\hline
\end{tabular}

\begin{tabular}{|c|c|}
\hline \multicolumn{2}{|c|}{ O-Muc-t72 } \\
\hline $\begin{array}{c}\text { Fold } \\
\text { change }\end{array}$ & $\begin{array}{c}\text { Abundance } \\
\text { (\%) }\end{array}$ \\
\hline$\downarrow$ & 7.28 \\
\hline$\uparrow$ & 3.87 \\
\hline$\uparrow$ & 8.82 \\
\hline$\downarrow$ & 2.72 \\
\hline$\uparrow$ & 2.09 \\
\hline$\uparrow$ & 0.03 \\
\hline$\uparrow$ & 0.13 \\
\hline$\downarrow$ & 0.18 \\
\hline$\uparrow$ & 0.16 \\
\hline$\uparrow$ & 0.23 \\
\hline$\downarrow$ & 0.09 \\
\hline$\uparrow$ & 0.04 \\
\hline$\uparrow$ & 24.58 \\
\hline$\uparrow$ & 0.02 \\
\hline$\downarrow$ & 0.004 \\
\hline$\uparrow$ & 2.42 \\
\hline$\uparrow$ & 5.67 \\
\hline$\uparrow$ & 0.74 \\
\hline$\uparrow$ & 0.78 \\
\hline & \\
\hline & \\
\hline & \\
\hline
\end{tabular}

\begin{tabular}{|c|c|}
\hline \multicolumn{2}{|c|}{ DC-Muc-t72 } \\
\hline $\begin{array}{c}\text { Fold } \\
\text { change }\end{array}$ & $\begin{array}{c}\text { Abundance } \\
\text { (\%) }\end{array}$ \\
\hline$\uparrow$ & 0.54 \\
\hline$\uparrow$ & 24.21 \\
\hline$\rightarrow$ & 0.82 \\
\hline$\uparrow$ & 32.95 \\
\hline$\uparrow$ & 4.80 \\
\hline$\downarrow$ & 0.004 \\
\hline$\uparrow$ & 0.36 \\
\hline$\uparrow$ & 0.02 \\
\hline$\uparrow$ & 1.21 \\
\hline$\uparrow$ & 0.15 \\
\hline$\uparrow$ & 0.78 \\
\hline$\uparrow$ & 0.11 \\
\hline$\downarrow$ & 23.79 \\
\hline$\uparrow$ & 0.04 \\
\hline$\uparrow$ & 0.0007 \\
\hline$\uparrow$ & 0.42 \\
\hline$\downarrow$ & 1.81 \\
\hline$\downarrow$ & 0.63 \\
\hline$\downarrow$ & 0.10 \\
\hline$\uparrow$ & 0.75 \\
\hline$\downarrow$ & 0.0004 \\
\hline
\end{tabular}


Table 4 shows the additional genera which were found to considerably change with respect to control in the L- and $\mathrm{O}$ - experiments after fermenting mucin for $72 \mathrm{~h}$, genera which were absent in the DC. Mucin stimulated the growth of Faecalibacterium, Dorea and Catenibacterium in the obese microbiota (2.12, 11.95 and 120.86 fold change with respect to control, respectively). In the lean microbiota of these groups both Faecalibacterium and Dorea were found to decrease (0.59 and 0.54 fold change with respect to control, respectively) and Catenibacterium was not detected.

Table 4. Relative change of bacterial genera after $72 \mathrm{~h}$ fermentation of mucin in TIM-2 with the complex communities. $\rightarrow$ indicates no change; $\uparrow$ indicates an increase; and $\downarrow$ indicates a decrease with respect to control. \% Values correspond to the relative abundance of the bacterial group in the mixtures at 772 . Cells with no value correspond to bacteria that were not detected while cells not containing an arrow represent groups that were detected in the intervention but not in the control, thus a fold change could be not estimated.

\begin{tabular}{|l|}
\hline Genus \\
\hline Lactococcus \\
\hline Streptococcus \\
\hline Citrobacter \\
\hline Faecalibacterium \\
\hline Eubacterium \\
\hline Intestinibacter \\
\hline Ruminococcus \\
\hline Enterobacter \\
\hline unclassified Eubacteriaceae \\
\hline Collinsella \\
\hline unclassified Coriobacteriaceae \\
\hline Allisonella \\
\hline Gemmiger \\
\hline Pediococcus \\
\hline Eggerthella \\
\hline Salmonella \\
\hline Turicibacter \\
\hline Negativicoccus \\
\hline Roseburia \\
\hline Dorea \\
\hline Fusicatenibacter \\
\hline Terrisporobacter \\
\hline Acinetobacter \\
\hline Senegalimassilia \\
\hline Peptostreptococcus \\
\hline Coprococcus \\
\hline Oscillospira \\
\hline unclassified Erysipelotrichaceae \\
\hline unclassified Mogibacteriaceae \\
\hline unclassified Prevotellaceae \\
\hline Parabacteroides \\
\hline Anaerostipes \\
\hline Catenibacterium \\
\hline
\end{tabular}
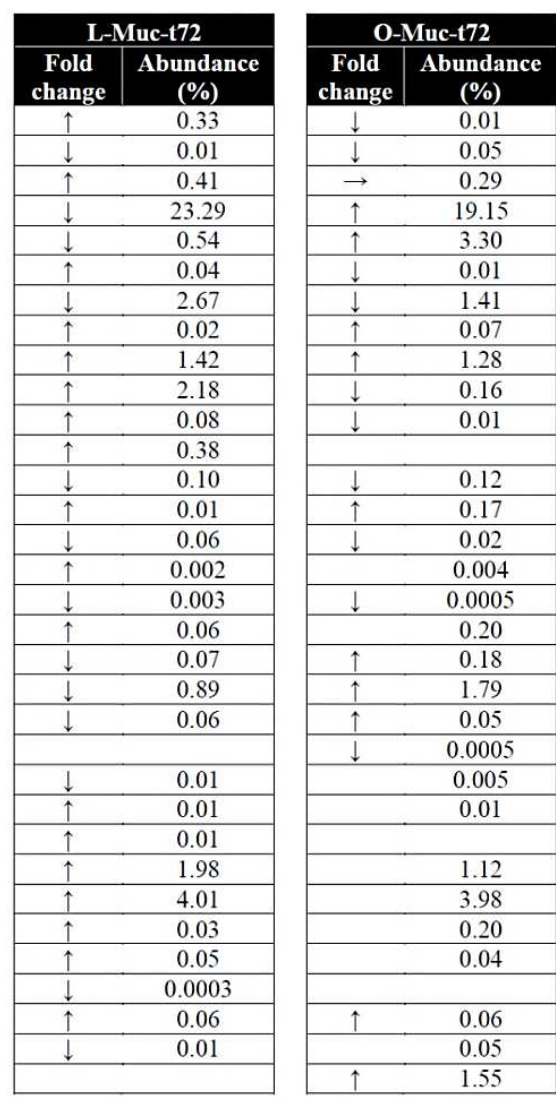


\section{Species level}

The sequencing analysis detected more than 14 strains in the DC samples (Table 5). Ten of the detected strains belonged to our inoculated consortium (grey cells) and represented $38 \%$ of the total reads.

Table 5. Relative change of species after $72 \mathrm{~h}$ of fermentation experiments of mucin in TIM- 2 in the DC- experiments. $\rightarrow$ indicates no change; $\uparrow$ indicates an increase; and $\downarrow$ indicates a decrease with respect to control. \% Values correspond to the relative abundance of the bacterial group in the mixtures at $\mathrm{t} 72$.

\begin{tabular}{|c|c|c|}
\hline \multirow[b]{2}{*}{ Species } & \multicolumn{2}{|c|}{ DC-Muc-t72 } \\
\hline & $\begin{array}{c}\text { Fold } \\
\text { change }\end{array}$ & \begin{tabular}{|c|}
$\begin{array}{c}\text { Abundance } \\
(\%)\end{array}$ \\
\end{tabular} \\
\hline Clostridium butyricum & $\downarrow$ & 2.75 \\
\hline Enterococcus faecium & $\uparrow$ & 22.11 \\
\hline unclassified Enterococcus & $\uparrow$ & 10.26 \\
\hline unclassified Clostridium & $\uparrow$ & 9.90 \\
\hline Bacteroides fragilis & $\rightarrow$ & 2.88 \\
\hline Unclassified & $\uparrow$ & 8.22 \\
\hline Bifidobacterium adolescentis & $\uparrow$ & 0.27 \\
\hline unclassified Lactobacillus & $\rightarrow$ & 0.35 \\
\hline Lactobacillus rhamnosus & $\uparrow$ & 0.23 \\
\hline unclassified Bacteroides & $\downarrow$ & 8.78 \\
\hline unclassified Bifidobacterium & $\uparrow$ & 0.18 \\
\hline Lactobacillus casei & $\downarrow$ & 0.23 \\
\hline Escherichia coli & $\uparrow$ & 0.24 \\
\hline Enterococcus ratti & $\uparrow$ & 0.38 \\
\hline Bacteroides vulgatus & $\downarrow$ & 2.24 \\
\hline unclassified Escherichia & $\uparrow$ & 0.12 \\
\hline Shigella sonnei & $\uparrow$ & 0.10 \\
\hline Bifidobacterium longum & $\uparrow$ & 0.09 \\
\hline Clostridium sporogenes & $\uparrow$ & 8.89 \\
\hline Enterococcus hirae & $\uparrow$ & 0.06 \\
\hline unclassified Shigella & $\uparrow$ & 0.02 \\
\hline Clostridium cadaveris & $\uparrow$ & 2.14 \\
\hline Enterococcus durans & $\uparrow$ & 0.03 \\
\hline Lactobacillus zeae & $\downarrow$ & 0.002 \\
\hline Bacteroides thetaiotaomicron & $\uparrow$ & 9.24 \\
\hline Enterococcus lactis & $\uparrow$ & 0.01 \\
\hline Lactobacillus paracasei & $\uparrow$ & 0.004 \\
\hline Enterococcus thailandicus & $\uparrow$ & 0.01 \\
\hline Enterococcus mundtii & $\downarrow$ & 0.0004 \\
\hline Bacteroides faecis & $\uparrow$ & 0.64 \\
\hline Enterococcus rivorum & $\uparrow$ & 0.003 \\
\hline $\begin{array}{l}\text { unclassified } \\
\text { Lachnoclostridium }\end{array}$ & $\uparrow$ & 0.28 \\
\hline Clostridium saccharolyticum & $\uparrow$ & 0.18 \\
\hline unclassified Blautia & $\downarrow$ & 1.09 \\
\hline Enterococcus casseliflavis & $\downarrow$ & 0.0004 \\
\hline unclassified Klebsiella & $\downarrow$ & 0.002 \\
\hline
\end{tabular}


The growth/decrease of the detected species in both complex communities was different (Table 6). However, when compared, both lean and obese microbiota coincided in the growth stimulation of an unclassified Clostridium (2.76 and 1.65 fold change with respect to control, respectively), L. mисоsae (8.22 and 3.41 fold change with respect to control, respectively), and an unclassified Lactobacillus ( 9.63 and 3.08 fold change with respect to control, respectively).

Table 6. Relative change of species after $72 \mathrm{~h}$ of fermentation experiments of mucin in TIM- 2 with the complex communities. $\rightarrow$ indicates no change; $\uparrow$ indicates an increase; and $\downarrow$ indicates a decrease with respect to control. \% Values correspond to the relative abundance of the bacterial group in the mixtures at $\mathrm{t} 72$. Cells with no value correspond to bacteria that were not detected while cells not containing an arrow represent groups that were detected in the intervention but not in the control, thus a fold change could be not estimated. 
Defined vs Complex communities

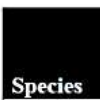

Clostridium butricicum Bifidobacterium adolescentis

unclassified Clostridium

unclassified Bifidobacterium

Lactobacillus mucosae

unclassified Lactobacillus

Unclassified

Bifidobacterium longum

Enterococcus faecium

Lactobacillus ruminis

unclassified Enterococcus

Faecalibacterium prausnitzii

unclassified Bacteroides

unclassified Faecalibacterium

Bacteroides vulgatus

Bacteroides fiagilis

Clostridium pelfingens

Enterococcus faecalis

Lactococcus lactis

unclassified Enbacterium

unclassified Lactococcus

Lactobacillus gasseri

unclassified Catenibacterium

Flavonifractor plautii

unclassified Flavonifractor

Intestinimonas butryiciproducens

unclassified Intestimimonas

unclassified Collinsella

Collinsella aerofaciens

unclassified Gemmiger

Blautia obeetm

Eubacterium ramulus

Pediococcus pentosaceus

Bifidobacterium bifidum

Holdemania massiliensis

unclassified Holdemania

Holdemania filiformis

unclassified Subdoligramulum

Ruminococcus faecis

unclassified Negativicoccus

Roseburia imulinivorans

Coprococcus catus

Blautia hansenii

Blautia luti

Ruminococcus torques

Atopobium minutum

unclassified Atopobium

Blautia producta

Clostridium hathervayi

Clostridium clostridioforme

unclassified Megasphaera

unclassified Psendoflavonifiactor

Dialister pneumosintes

\begin{tabular}{|c|c|}
\hline \multicolumn{2}{|c|}{ L-Muc-772 } \\
\hline $\begin{array}{c}\text { Fold } \\
\text { change }\end{array}$ & $\begin{array}{c}\text { Abundance } \\
(\%)\end{array}$ \\
\hline
\end{tabular}

change (\%)

\begin{tabular}{|cc|}
\hline$\uparrow$ & 1.52 \\
\hline$\downarrow$ & 0.39 \\
\hline
\end{tabular}

\begin{tabular}{ll}
1 & 0.39 \\
\hline$\uparrow$ & 2.08 \\
\hline & 7.27
\end{tabular}

\begin{tabular}{|ll|}
\hline$\uparrow$ & 7.27 \\
\hline$\uparrow$ & 1.54 \\
\hline
\end{tabular}

\begin{tabular}{ll}
\hline$\uparrow$ & 0.79 \\
\hline$\uparrow$ & 9.35 \\
\hline
\end{tabular}

\begin{tabular}{rr}
$\uparrow$ & 0.56 \\
\hline & 1.72
\end{tabular}

\begin{tabular}{ll}
$\downarrow$ & 0.30 \\
\hline & 1.72 \\
\hline
\end{tabular}

\begin{tabular}{|cc|}
\hline$\downarrow$ & 0.42 \\
\hline$\downarrow$ & 15.32 \\
\hline$\downarrow$ & 3.78 \\
\hline
\end{tabular}

\begin{tabular}{|ll|}
\hline$\downarrow$ & 3.78 \\
\hline$\downarrow$ & 7.61 \\
\hline$\downarrow$ & 0.93 \\
\hline
\end{tabular}

\begin{tabular}{lll}
$\uparrow$ & 0.66 \\
\hline$\uparrow$ & 0.93 \\
\hline
\end{tabular}

\begin{tabular}{ll}
$\uparrow \uparrow$ & 0.33 \\
\hline$\uparrow$ & 0.66
\end{tabular}

\begin{tabular}{ll}
$\uparrow$ & 0.66 \\
\hline$\uparrow$ & 0.43 \\
\hline
\end{tabular}

\begin{tabular}{|ll|}
\hline$\uparrow$ & 0.41 \\
\hline$\uparrow$ & 0.22 \\
\hline$\uparrow$ & \\
\hline
\end{tabular}
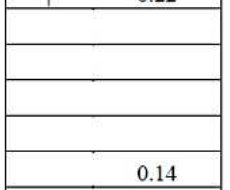

\begin{tabular}{|c|}
\hline 0.14 \\
\hline 0.09 \\
\hline 0.83 \\
\hline 1.25 \\
\hline 0.13 \\
\hline
\end{tabular}
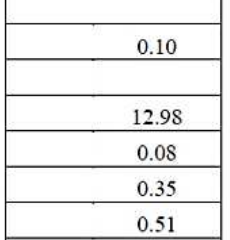

0.51

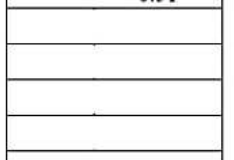

O-Muc-172

Fold Abundance

change (\%)

\begin{tabular}{cc}
\hline$\downarrow$ & 0.75 \\
\hline$\downarrow$ & 0.24 \\
\hline
\end{tabular}

$\uparrow \quad 1.54$

\begin{tabular}{ll}
$\downarrow$ & 2.89 \\
$\uparrow$ & 5.32 \\
\hline
\end{tabular}

$\begin{array}{ll}\uparrow & 2.88 \\ \uparrow & 8.35\end{array}$

$\begin{array}{rr}\downarrow & 1.61 \\ \downarrow & 1.53\end{array}$

$\downarrow \quad 1.00$

\begin{tabular}{|r|}
\hline 13.87 \\
\hline 8.47 \\
\hline
\end{tabular}

8.37
5.37
12.71

\begin{tabular}{|l|}
\hline 0.49 \\
\hline 0.27 \\
\hline
\end{tabular}

0.20

$\uparrow \quad 1.40$

$\uparrow \quad 0.62$

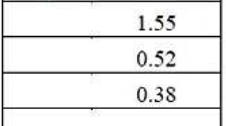

0.38

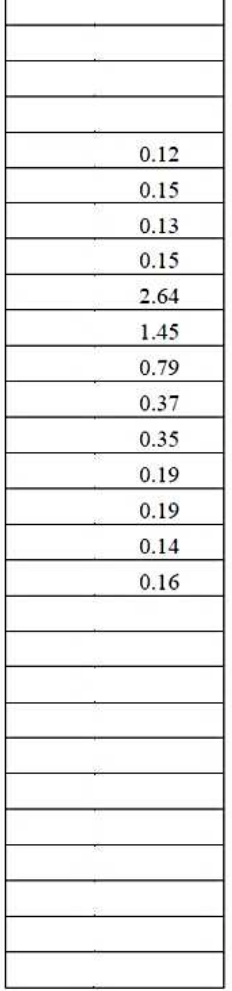


Gene expression

The hierarchical clustering analysis (Fig. S1) showed a difference among the Obese/Lean/DC samples and the time effect (samples split: all t0 and t72). It was also possible to observe that there were two outliers: sample \#8 (Obese, Control, t72) and \#18 (DC, Mucin, t72). The results from the correlation analysis (Fig. S2) were similar when compared with the clustering: i) all the t0 samples ( $\mathrm{L}$ and $\mathrm{O}$ ) were similar. Obese was placed in one block and lean in other block but generally they were similar and after $72 \mathrm{~h}$ they started to re-accommodate in another cluster; ii) DC was very different from the rest of the samples. For the outliers: \#8 did not correlate with anything suggesting that it was very different when compared to the others. The RNA in this sample appeared to be degraded.

The PCA analysis (Fig. S3; PC1 28.14\% of all variation and PC2 20.14\% of variation) was in agreement with the hierarchical clustering: i) DC samples were different from $\mathrm{L}$ and $\mathrm{O}$ with one exception: sample \#8; ii) t0 clustered separately from 172 (expected). However, there was something new which was possible to observe from this analysis: all samples tagged with numbers above 20 tended to form a separate group. This was due to two different hybridization experiments. Normalization will be carried out to correct for this.

The analysis of the differentially expressed probes showed that only 3 genes were differentially expressed in both the DC and the two complex communities (i.e., lean and obese; Table S2).

In the heat map (Fig. 3), the differentially expressed genes were grouped per pathway and in some cases they were present i) in multiple pathways, or ii) multiple times in the same pathway.

Mucin was found to have an effect on the expression of genes encoding enzymes involved in glycosaminoglycan degradation; valine, leucine and isoleucine biosynthesis; pyruvate metabolism; propanoate metabolism; butanoate metabolism; and pentose phosphate pathway. Our heatmap revealed an interesting contrast between the lean and obese communities in the expression of different genes by $F$. prausnitzii, B. fragilis, B. thetaiotaomicron, B. vulgatus, and B. longum subs. infantis (Fig. 3). Some genes were downregulated or upregulated in the lean community while it seemed to be the opposite in the obese community. These genes were found to be associated with glycosaminoglycan degradation, pyruvate and propanoate metabolism. 
Defined vs Complex communities

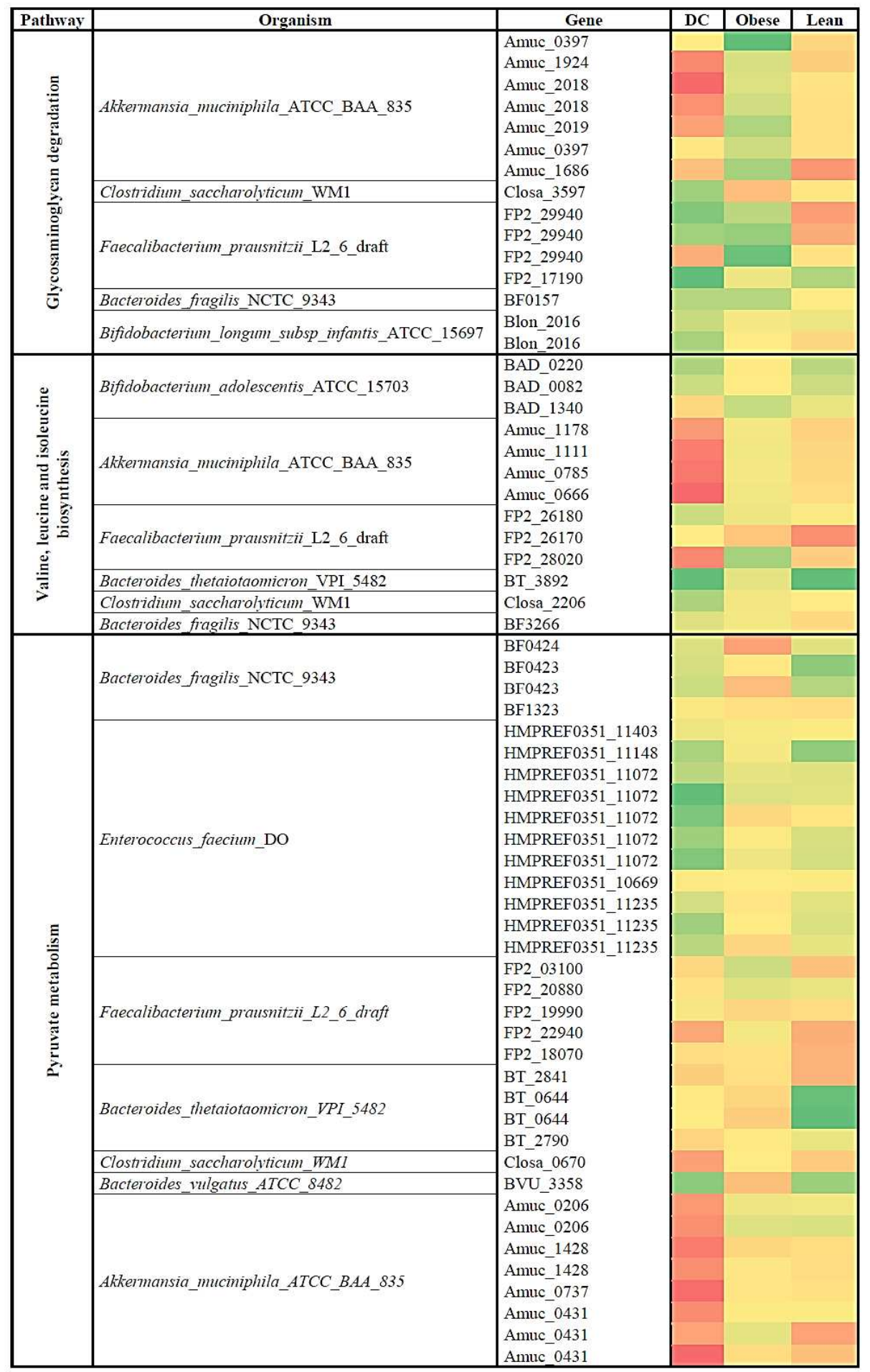




\begin{tabular}{|c|c|c|c|c|c|}
\hline Pathway & Organism & Gene & DC & Obese & Lean \\
\hline \multirow{22}{*}{ 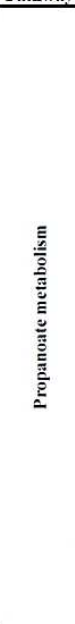 } & & \begin{tabular}{|l|}
$\mathrm{BF} 0424$ \\
$\mathrm{BF} 0423$
\end{tabular} & & & \\
\hline & & $\mathrm{BF} 0423$ & & & \\
\hline & & BF 1323 & & & \\
\hline & Bacteroides_fragilis_NCTC_9343 & BF1050 & & & \\
\hline & & $\mathrm{BF} 3128$ & & & \\
\hline & & $\mathrm{BF} 3128$ & & & \\
\hline & & BF2351 & & & \\
\hline & & $\begin{array}{l}\text { BF2351 } \\
\text { Amuc 1983 }\end{array}$ & & & \\
\hline & & $\begin{array}{l}\text { Amuc__1983 } \\
\text { Amuc } 1428\end{array}$ & & & \\
\hline & & Amuc 1428 & & & \\
\hline & Akkermansia_muciniphila_ATCC_BAA_S35 & Amuc_2034 & & & \\
\hline & & Amuc_2034 & & & \\
\hline & & $\begin{array}{l}\text { Amuc_2034 } \\
\text { Amuc 2034 }\end{array}$ & & & \\
\hline & & HMPREF 0351_11403 & & & \\
\hline & & HMPREF 0351 1 11072 & & & \\
\hline & & $\begin{array}{l}\text { HMPREF0351_11072 } \\
\text { HMPREF0351_11072 }\end{array}$ & & & \\
\hline & Enterococcus_faecium_DO & HMPREF0351_11072 & & & \\
\hline & & HMPREF0351_11072 & & & \\
\hline & & $\begin{array}{l}\text { HMPREF0351_11148 } \\
\text { HMPREF0351 } 11403\end{array}$ & & & \\
\hline & & FP2 20880 & & & \\
\hline & Faecalibacterium_prausmitzii_L2_6_draft & FP2_-03100 & & & \\
\hline & Bifidobacterium_longum_subsp_infantis_ATCC_15697 & Blon_2285 & & & \\
\hline \multirow{14}{*}{ 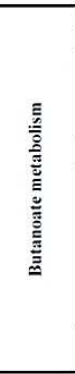 } & Bifidobacterium adolescentis ATCC 15703 & \begin{tabular}{|l|l|} 
BAD_0220 \\
\end{tabular} & & & \\
\hline & 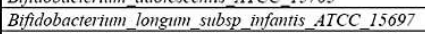 & Blon_2239 & & & \\
\hline & & Amuc_0204 & & & \\
\hline & L thone & Amuc_0204 & & & \\
\hline & Akkermansia_muciniphila_ATCC_BAA_S35 & Amuc_0204 & & & \\
\hline & Bacternides fingilis NCTC 0243 & Amuc_0204 & & & \\
\hline & Bacteroides fraglilis_NCTC_9343 & $\begin{array}{l}\mathrm{BF} 1323 \\
\text { HMPREF } 035111072\end{array}$ & & & \\
\hline & & HMPREF0351_11072 & & & \\
\hline & & HMPREF0351_11072 & & & \\
\hline & Enterococcus_faecium_DO & HMPREF0351_11072 & & & \\
\hline & & HMPREF0351_11072 & & & \\
\hline & & HMPREF0351_11148 & & & \\
\hline & Raccernides thetaint & BT_3054 & & & \\
\hline & Bacteroides_thetaiotaomicron_VPI__4SS2 & BT 3054 & & & \\
\hline \multirow{24}{*}{ 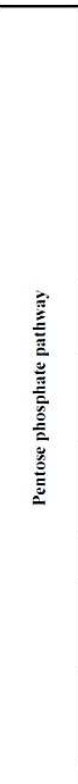 } & & $\begin{array}{l}\text { HMPREF0351_10290 } \\
\text { HMPREF0351_10625 }\end{array}$ & & & \\
\hline & & HMPREF0351_11798 & & & \\
\hline & & HMPREF0351_-10287 & & & \\
\hline & & $\begin{array}{l}\text { HMPREF0351_10419 } \\
\text { HMPREF0351_10045 }\end{array}$ & & & \\
\hline & Enterococcul_faecium_DO & HMPREF0351_10551 & & & \\
\hline & & HMPREF0351_10551 & & & \\
\hline & & HMPREF 0351_11311 & & & \\
\hline & & HMPREF0351_11526 & & & \\
\hline & & $\begin{array}{l}\text { HMPREF0351_10045 } \\
\text { BAD } 0793\end{array}$ & & & \\
\hline & Bifidobacterium_adolescentis_ATCC_15703 & BAD_0793 & & & \\
\hline & & $\begin{array}{l}\text { BAD_0628 } \\
\text { Closa } 1510\end{array}$ & & & \\
\hline & Clostridium saccharolyticum WVI & Closa_3978 & & & \\
\hline & Clostridium_saccharolyticum_WMI & Closa_1233 & & & \\
\hline & & $\begin{array}{l}\text { Closa_1510 } \\
\text { FP2 } 30650\end{array}$ & & & \\
\hline & Faecalibacterium prousmitivi L2 6 draft & FP2_30650 & & & \\
\hline & & $\begin{array}{l}\mathrm{FP} 232120 \\
\mathrm{FP} 2-25460\end{array}$ & & & \\
\hline & Akkernnansia_muciniphila_ATCC_BAA_S35 & Amuc_1653 & & & \\
\hline & & $\begin{array}{l}\text { Amuc_o/21 } \\
\text { BF1921 }\end{array}$ & & & \\
\hline & Bacteroides fragilis_NCTC 9343 & BF 3604 & & & \\
\hline & & $\begin{array}{l}\mathrm{BF} 3604 \\
\mathrm{BF} 3604\end{array}$ & & & \\
\hline & & BT_2124 & & & \\
\hline & Bacteroides thetaiotaomicron VPI $54 S 2$ & BT_2124 & & & \\
\hline & 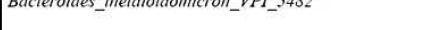 & BT_2124 & & & \\
\hline & Streptococcus therpophilus LMG 18311 & BT_2124 & & & \\
\hline
\end{tabular}

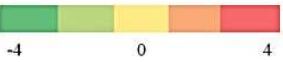


Fig. 3. Heat map. The data from the heat map is $2 \mathrm{Log}$ ratio and correspond to the subtraction Muc-Control at $72 \mathrm{~h}$. The intensity of the colors indicates upregulation (red) or downregulation (green).

\section{Discussion}

Some species in the human gut have the ability to use mucus glycans (directly or indirectly) as a carbon source to grow [40-42]. Yet, this is estimated to be a low proportion (around 1\% of the total colonic species). Mucin is a major constituent of the mucus layer in the gastrointestinal tract [43-45]. Not surprisingly, it has been estimated that around 3 to 5 grams of mucin and related host-associated glycoproteins are used as a substrate for bacterial fermentation every day [46,47]. Still, a limited number of studies have addressed how changes in host glycan landscape make an impact on gut microbial functionality and composition [48].

It is believed that host-glycans play an important role in functionality and stability of the gut microbiota. Reasons supporting this important trait are based on the fact that $[8,40]$ : i) mucus can be potentially used as a niche for specific groups of bacteria (e.g. Bacteroidetes spp., Akkermansia muciniphila) remaining present in the gut ecosystem by avoiding their washout; ii) glycans represent an abundant constant source of nutrients for some bacteria; iii) sugars liberated from the breakdown of mucus and other products from its fermentation generated by mucusassociated bacteria could be utilized by other microorganisms in the gut in crossfeeding. For the latter we speculate that it could be of key importance for microbiota stability during fasting of the host.

Currently, there are a number of in vitro gut models that simulate the gut microbiota. The majority of these models do not contain the addition of mucin, which results only in the simulation of luminal microbiota, excluding microorganisms which profit from a mucosal environment to grow optimally.

This lack of mucin makes it more challenging in achieving a representative simulation of the human gut compared to the in vivo situation. In the current study, mucin was provided to three different communities (i.e. defined community, and lean and obese fecal microbiota) in order to evaluate the role of this component in the development (metabolically and compositionally) of the bacteria in an in vitro system simulating the human gut.

\section{Metabolic activity and energy extraction}

When compared to their respective controls, differences compared to control in both kinetics and quantitative metabolite production by the three communities were observed to be driven by mucin. However, the shifts induced by the presence of 
mucin were also different when the communities were compared among each other. This indicates that microbial communities might present substrate specificity to a different degree, depending on the presence of mucin (Fig. 1, Table 2). This was also observed in the compositional analysis as discussed later.

Remarkably, the total cumulative production of SCFA in the mucin experiments was about as high as in the control experiments for all three communities (but differed between communities). This confirmed that microbes in a mixed coculture are engineered to interact by sharing degraded glycan-components or metabolites in cross-feeding, exhibiting dynamics of complementary metabolism as previously suggested [49].

Not all microorganisms in the gut are properly equipped for degrading mucin. The ones that are capable possess glucosidases which degrade mucin molecules into monosaccharides and amino acids which could be released in the surrounding environment and used by less specialized bacteria [50,51]. Yet, the efficiency of such cooperation dynamics depends on how easily the exchange of nutritional components or metabolites occurs. It has been observed that in some cases the efficiency of dietary polysaccharides fermentation is boosted in some species by the presence of others $[8,52]$. On the other hand, the growth of some groups could be inhibited when added in a co-culture [51]. This is not surprising, microbes in the human gut live under a selective pressure characterized by fierce competition among members in an overpopulated community ranging from $10^{11}$ to $10^{12}$ organisms per gram of luminal content [8] and sometimes not everything is about cooperation, it is also about survival. By just adding mucin, a synergetic activity is induced which has an impact on the fermentation activity of bacteria, especially on the less competitive groups whose survival becomes more challenging due to their limited capability of using mucus to grow [53-55].

An efficient cooperation among microbes implies that donor microorganisms, of metabolites or degraded substrates, are more likely to share growth-cheap (low value) metabolites with recipients demanding less energy investment [49]. The steady increment of SCFA as observed in the kinetics from the complex communities (i.e. lean and obese microbiota) suggest a more efficient interaction compared to the DC which despite of producing a cumulative value almost as high as its respective control exhibited a slow starting production of metabolites (Fig. 1). This is in agreement with the study from Willis et al. [56] who found that fecal bacteria are much more efficient in degrading and metabolizing mucin when compared to pure cultures.

The metabolite production and kinetics from the DC indicated that this consortium is still far from representing a complex community such as the one found in the gut from lean or obese individuals. Still, what is remarkable from these results is that 
despite the uniqueness of each microbial species included in the DC in terms of individual enzyme machinery and therefore metabolism, tasks, etc., there were certain synergy actions together as a group which was reflected in terms of metabolic activity and energy extraction. Not only the relatively high total cumulative amounts of metabolites for the DC were surprising, but also it corroborates the metabolic differences evident among intestinal communities. Clear differences were identified in the metabolic profiles of the communities studied, this encourages to explore more in the identification of distinctive health markers as pointed out by Yen et al. [57], especially in the context of obesity. Another interesting point is that despite a low abundance of Akkermansia, several genes were expressed under the experimental conditions. One of these, Amu_0666 (encoding an enzyme involved C5-Branched dibasic acid metabolism), was differentially affected by mucin all in three communities. However, at this moment the results should be interpreted with some care, as the array and RT-q-PCR data do not match for all samples (data not shown).

The addition of mucin to the growth media, particularly in the defined and the lean communities, caused an elevated production of BCFA when compared to their respective controls and the obese community. This shows evidence that mucins as glycosylated proteins can potentially change the fermentation metabolites of bacterial populations [55]. However, the media containing mucin used for this study had a higher content of protein when compared to the control media. Therefore, this could have influenced the results. Still, the fermentable protein available in the mucin containing media was the same in all the experiments with mucin, also for the obese microbiota. Further research is needed in order to elucidate what the impact of the higher production of BCFA, normally regarded as potentially toxic from proteolytic fermentation, may be on host health.

Under the conditions tested, the extraction of energy was higher in the lean microbiota when compared to the other communities (Fig. 2). Suggesting, once again (Chapters 6 and 7), that the energy derived from the fermentation of indigestible compounds may not only be microbiota but certainly is also substrate dependent as previously observed by us when testing the energy extraction from different prebiotics in lean and obese microbiota [58].

\section{Composition}

The presence of mucin did favor the increase in beneficial bacterial abundance such as Lactobacillus and Bifidobacterium in the studied communities.

Lactobacillus was observed to increase in both lean and obese communities whilst it did not change in the DC after the incorporation of mucin in the media (Table 3). In both lean and obese L. mucosae increased considerably with respect to control 
(8.22 and 3.41 fold change respectively) (Table 6). This species was not present in the DC community.

Our findings are in agreement with Van den Abbeele et al. [10] who observed that incorporating a mucosal environment in an in vitro model improved the growth of L. mucosae. This species was detected in the luminal environment when mucin was administrated to the dynamic model simulating the human gut (SHIME) [10]. However, it has also been found, both in vitro and in vivo, that it can attach to epithelial cells, which suggests that this microorganism could have the ability to interact with the host, potentially influencing the host's immune system $[10,59]$. In our studies we also confirmed the mucolytic activity from B. bifidum on porcine mucin, which, judging from the relative abundance from this species (t72), was more efficient in the lean community when compared to the obese (12.98 and $2.64 \%$, respectively). The genome sequencing of B.longum showed that this species has a higher carbohydrate degradation capacity than lactobacilli [60]. However, the growth of B. longum can be affected by Bacteroides [61].

The effect of mucin on Lactobacillus and Bifidobacterium suggests that the addition of mucin to an in vitro model simulating the human gut affects the development of specific bacteria which could be dependent on an ecosystem and which requires mucus in order to develop optimally. It should be mentioned however that from our results this seems to be restricted to selected species within the two genera, namely L. mucosae and B.bifidum. Furthermore, this observation is interesting in the context of investigating the effect of probiotics in the human gut. Incorporating mucin in an in vitro model testing the performance of a probiotic may help to elucidate better how the colonization and the functionality of the tested bacteria in an existing community is under optimal conditions. We speculate that there may be a difference in the colonization and metabolic activity when a probiotic, able to use mucin as an energy source, is introduced to a mucin free environment. Therefore, if the latter happens, we hypothesize that the response of the probiotic to this new environment may be a stress response which implies some bias in the outcome.

Enterococcus was observed to be benefited in the DC upon feeding mucin. This could be explained by a possible cooperation with other groups of bacteria which could have facilitated their growth. Enterococci have been suggested to gain some nutritional support from Bacteroides species and Bifidobacterium species after being found on biofilms formed on the surface of food particles in which these groups have also been found to be present [45,62]. Furthermore, Pultz et al. [45] showed that although some E. faecium strains were unable to metabolize bovine submaxillary mucin or hog gastric mucin, they could ferment metabolites derived from this substrate. This was evident in the DC experiments where this species 
increased (1.81 fold change with respect to control). The fact that Enterococcus species have been shown to facilitate digestion of proteins by the production of proteases could also explain the high cumulative amounts of BCFA produced in these experiments [63].

Members of Escherichia were seen to increase in all communities. Despite that this species does not produce enzymes capable of degrading polysaccharides it has been observed that at least in the colonization of the gastrointestinal system of the mouse it can harvest energy from mucosal glycoproteins or monossacharides derived from mucin $[45,64]$.

As indicated in the analysis at the genus level, the Bacteroides group from the obese microbiota particularly benefited by the addition of mucin to the media ( 6.27 fold change with respect to control; Table 3). This genus has been observed to rapidly colonize mucus [13]. The growth of species belonging to the Bacteroides species was found in the mucin experiments but not in the control. Species identified were B. vulgatus and B. fragilis (Table 6). In the case of B. vulgatus it has been observed that monocultures of this species have previously shown some mucolytic activity after the administration of porcine mucin [51]. Our study showed that this species was one of the most abundant in the obese community after adding mucin. B. fragilis also benefited by this substrate though its abundance was relatively low probably due to competition with the other members of the community. Huan et al. [65] found that $B$. fragilis can grow in the luminal colon of gnotobiotic mice as well as it can bind to intestinal mucin in in vitro assays. Therefore, it would be interesting to further explore in detail how these species are involved and the consequences of such colonization in the obese microbiota.

Interestingly, although overall the genus Bacteroides in the DC group was not observed to increase, when this genus was checked at the level of species we found that $B$. thetaiotaomicron increased after the addition of mucin (1.88 fold change with respect to control). Previous functional genomic studies focusing on the responses of B. thetaiotaomicron to co-culture with $L$. casei or B. longum in a gnotobiotic mice found that $B$. thetaiotaomicron can easily adapt to the presence of other bacteria due to its metabolic flexibility $[8,66]$. There is evidence indicating that depending on the availability of dietary or host polysaccharides, $B$. thetaiotaomicron can harvest energy, however it presents some preference for dietary polysaccharides [42].

Blautia and Roseburia genera increased in the fermentations with the obese microbiota. The Lachnospiraceae family includes the Roseburia and Blautia genera which are known to be involved in the transformation of carbohydrates to SCFA [67]. These genera have been considered key members of a balanced microbiota since their decrease declines the levels of SCFA production, especially 
butyrate, compromising the intestinal barrier $[68,69]$. There is evidence indicating that butyrate is involved in the induction of mucin synthesis [70]. Roseburia species have been seen to increase after the inclusion of mucus in another in vitro model [71].

The presence of $F$. prausnitzii is considered as an indicator of a healthy gastrointestinal tract [72]. It is estimated that its relative abundance is around the $25 \%$ of all bacteria in a healthy human gut [73]. Interestingly, the abundance of this species has been found to be reduced in obese individuals and has also been negatively correlated with inflammatory markers [74]. In our study, F. prausnitzii increased in the experiments with the obese community after adding mucin to the media ( 2.12 fold change with respect to the control; Table 4). Given the fact that the evidence about the use of mucin as energy source by this species is scarce $[29,75]$, we believe that the addition of mucin in in vitro studies particularly focusing on obesity could be of great value.

Members from the Oscillospira genus were detected in both lean and obese microbiota. This genus has been negatively correlated with BMI. Tims et al. [76] found a high abundance of $O$. guillermondii in siblings with a low BMI. These authors speculated that the presence of members from the Oscillospira genus may be involved on the metabolism of nutritional fibers. Still, there is a lack of studies investigating the presence of this bacteria and their potential role in energy homeostasis, requiring more research.

Akkermansia muciniphila is currently one of the most studied mucin degrading bacteria. Interestingly, A. muciniphila is suspected to play an important role in metabolic disorders and inflammatory bowel diseases [77]. Despite that there is evidence that A. muciniphila can degrade both human (MUC2) and porcine gastric mucus (MUC5AC) [78,79] the growth of this bacterium was not really stimulated in any of our experiments. However, this is not the first time that A. muciniphila is observed not to grow even with a mucus source added to the media. Png et al. [51] found that $A$. muciniphila was unable to grow in co-culture. Yet, it did facilitate the growth of other bacteria studied: $B$. vulgatus and B. bifidum. It may be that growing in vitro in complex communities (e.g. lean and obese) demanded much more competition for A. muciniphila when compared to in vivo. Though less complex, the growth in a DC may also be challenging for the bacteria. Of note, we did observe gene expression of gene in Akkermansia as discussed below.

\section{Gene expression}

The analysis of the differentially expressed probes showed that only 3 genes were differentially expressed in both the DC and the two complex communities (i.e., lean and obese) (Table S2). Although many more genes were also expressed they 
did not follow the same expression pattern in the three communities. We expected a low abundance of differentially expressed genes among the three communities. As previously found, there are household genes in the microbiota that are expressed similarly under multiple different conditions [80] which underscores the high degree of similarity on functional genomic profiles. We speculate from the fact that there were more differentially expressed probes detected in the lean community (4414) in comparison to DC (2300) and obese (1457) that the microbiota from lean subjects may be better equipped to the degradation of host's glycans.

Our heatmap revealed an interesting contrast between the lean and obese communities in the expression of different genes by $F$. prausnitzii, B. fragilis, $B$. thetaiotaomicron, B. vulgatus, and B. longum subs. infantis (Fig. 3). Some genes were downregulated or upregulated in the lean community while they seemed to be the oppositely expressed in the obese microbiota. These genes were found to be associated with glycosaminoglycan degradation, pyruvate and propanoate metabolism. However, after the normalization of the data this needs to be further explored.

\section{Conclusion}

Our results illustrate how a simplified defined microbial community is useful for the understanding of the dynamics that shape the human gut microbiota in terms of metabolite production, compositional changes and gene expression. However more efforts are needed in order to find the correct balance of the members of such defined community and promote their growth when they are all incorporated in the in vitro system.

The present study highlights how some bacterial groups (from both defined and complex communities) are able to shift their substrate utilization as a response to dietary changes (as previously presented in Chapter 5-7). Furthermore, the addition of mucin in an in vitro model gives room to speculate how host physiology can affect the functionality and composition of the microbiota increasing the opportunity of identifying potential health markers. Moreover, a model that incorporates mucin during its operation will be particularly useful when studying the response of the gut microbiota during prebiotic, probiotic and antibiotic treatment. 
Chapter 8

\section{Acknowledgments}

This study was partly funded by the Top Institute Food \& Nutrition (TIFN, Wageningen, The Netherlands; GH004). We thank Carlota Bussolo de Souza for providing the lean and obese inocula and Hermie Harmsen for providing the $F$. prausnitzii strain for the DC experiments. 
Defined vs Complex communities

\section{References}

1. Clemente JC, Ursell LK, Parfrey LW, Knight R (2012) The impact of the gut microbiota on human health: an integrative view. Cell 148: 1258-1270.

2. Ramakrishna BS (2013) Role of the gut microbiota in human nutrition and metabolism. J Gastroenterol Hepatol 28: 9-17.

3. Roeselers G, Bouwman J, Venema K, Montijn R (2012) The human gastrointestinal microbiota an unexplored frontier for pharmaceutical discovery. Pharmacol Res 66: 443-447.

4. Eckburg PB, Bik EM, Bernstein CN, Purdom E, Dethlefsen L, et al. (2005) Diversity of the human intestinal microbial flora. Science 308: 1635-1638.

5. McNulty NP, Wu M, Erickson AR, Pan C, Erickson BK, et al. (2013) Effects of Diet on Resource Utilization by a Model Human Gut Microbiota Containing Bacteroides cellulosilyticus WH2, a Symbiont with an Extensive Glycobiome. PLoS Biol 11: e1001637.

6. Faith JJ, McNulty NP, Rey FE, Gordon JI (2011) Predicting a human gut microbiota's response to diet in gnotobiotic mice. Science 333: 101-104.

7. Morgan JL, Darling AE, Eisen JA (2010) Metagenomic sequencing of an in vitrosimulated microbial community. PLoS One 5: e10209.

8. Mahowald MA, Rey FE, Seedorf H, Turnbaugh PJ, Fulton RS, et al. (2009) Characterizing a model human gut microbiota composed of members of its two dominant bacterial phyla. Proc Natl Acad Sci U S A 106: 5859-5864.

9. Derrien M, Van Baarlen P, Hooiveld G, Norin E, Muller M, et al. (2011) Modulation of Mucosal Immune Response, Tolerance, and Proliferation in Mice Colonized by the Mucin-Degrader Akkermansia muciniphila. Front Microbiol 2: 166.

10. Van den Abbeele P, Roos S, Eeckhaut V, MacKenzie DA, Derde M, et al. (2012) Incorporating a mucosal environment in a dynamic gut model results in a more representative colonization by lactobacilli. Microb Biotechnol 5: 106-115.

11. Zoetendal EG, von Wright A, Vilpponen-Salmela T, Ben-Amor K, Akkermans AD, et al. (2002) Mucosa-associated bacteria in the human gastrointestinal tract are uniformly distributed along the colon and differ from the community recovered from feces. Appl Environ Microbiol 68: 3401-3407.

12. Probert HM, Gibson GR (2004) Development of a fermentation system to model sessile bacterial populations in the human colon. Biofilms 1: 13-19.

13. Macfarlane S, Woodmansey EJ, Macfarlane GT (2005) Colonization of mucin by human intestinal bacteria and establishment of biofilm communities in a twostage continuous culture system. Appl Environ Microbiol 71: 7483-7492.

14. Minekus M, Smeets-Peeters M, Bernalier A, Marol-Bonnin S, Havenaar R, et al. (1999) A computer-controlled system to simulate conditions of the large 
intestine with peristaltic mixing, water absorption and absorption of fermentation products. Appl Microbiol Biotechnol 53: 108-114.

15. Luo J, Zheng A, Meng K, Chang W, Bai Y, et al. (2013) Proteome changes in the intestinal mucosa of broiler (Gallus gallus) activated by probiotic Enterococcus faecium. J Proteomics 91: 226-241.

16. Wrzosek L, Miquel S, Noordine ML, Bouet S, Joncquel Chevalier-Curt M, et al. (2013) Bacteroides thetaiotaomicron and Faecalibacterium prausnitzii influence the production of mucus glycans and the development of goblet cells in the colonic epithelium of a gnotobiotic model rodent. BMC Biol 11: 61.

17. Ruseler-van Embden JG, van der Helm R, van Lieshout LM (1989) Degradation of intestinal glycoproteins by Bacteroides vulgatus. FEMS Microbiol Lett 49: 3741.

18. Le KA, Li Y, Xu X, Yang W, Liu T, et al. (2012) Alterations in fecal Lactobacillus and Bifidobacterium species in type 2 diabetic patients in Southern China population. Front Physiol 3: 496.

19. Miquel S, Martin R, Rossi O, Bermudez-Humaran LG, Chatel JM, et al. (2013) Faecalibacterium prausnitzii and human intestinal health. Curr Opin Microbiol 16: 255-261.

20. Duncan SH, Flint HJ (2008) Proposal of a neotype strain (A1-86) for Eubacterium rectale. Request for an opinion. Int J Syst Evol Microbiol 58: 1735-1736.

21. Qiu X, Zhang M, Yang X, Hong N, Yu C (2013) Faecalibacterium prausnitzii upregulates regulatory $\mathrm{T}$ cells and anti-inflammatory cytokines in treating TNBS-induced colitis. J Crohns Colitis 7: e558-568.

22. Hols P, Hancy F, Fontaine L, Grossiord B, Prozzi D, et al. (2005) New insights in the molecular biology and physiology of Streptococcus thermophilus revealed by comparative genomics. FEMS Microbiol Rev 29: 435-463.

23. Fichera GA, Giese G (1994) Non-immunologically-mediated cytotoxicity of Lactobacillus casei and its derivative peptidoglycan against tumor cell lines. Cancer Lett 85: 93-103.

24. Linko S, Enwald S, Zhu YH, Mäyrä-Mäkinen A (1998) Production of Bgalactosidase by Streptococcus salivarius subsp thermophilus 11F. J Ind Microbiol Biotechnol 20: 215-219.

25. You J, Yaqoob P (2012) Evidence of immunomodulatory effects of a novel probiotic, Bifidobacterium longum bv. infantis CCUG 52486. FEMS Immunol Med Microbiol 66: 353-362.

26. Khan AA, Khurshid M, Khan S, Alshamsan A (2013) Gut Microbiota and Probiotics: Current Status and Their Role in Cancer Therapeutics. Drug Dev Res 74: 365-375.

27. Peloquin JM, Nguyen DD (2013) The microbiota and inflammatory bowel disease: insights from animal models. Anaerobe 24: 102-106. 
Defined vs Complex communities

28. Zhang Y, Wang L, Zhang J, Li Y, He Q, et al. (2014) Probiotic Lactobacillus casei ameliorates high-fructose-induced impaired glucose tolerance in hyperinsulinemia rats. Eur J Nutr 53: 221-232.

29. Lopez-Siles M, Khan TM, Duncan SH, Harmsen HJ, Garcia-Gil LJ, et al. (2012) Cultured representatives of two major phylogroups of human colonic Faecalibacterium prausnitzii can utilize pectin, uronic acids, and host-derived substrates for growth. Appl Environ Microbiol 78: 420-428.

30. Duncan SH, Hold GL, Harmsen HJ, Stewart CS, Flint HJ (2002) Growth requirements and fermentation products of Fusobacterium prausnitzii, and a proposal to reclassify it as Faecalibacterium prausnitzii gen. nov., comb. nov. Int J Syst Evol Microbiol 52: 2141-2146.

31. Aguirre M, Ramiro-Garcia J, Koenen ME, Venema K (2014) To pool or not to pool? Impact of the use of individual and pooled fecal samples for in vitro fermentation studies. J Microbiol Methods 107C: 1-7.

32. Aguirre M, Eck A, Koenen ME, Savelkoul PHM, Budding AE, et al. (2015) Evaluation of an optimal preparation of human standardized fecal inocula for in vitro fermentation studies. J Microbiol Methods 117: 78-84.

33. van Nuenen MHMC, Diederick Meyer P, Venema K (2003) The Effect of Various Inulins and Clostridium difficile on the Metabolic Activity of the Human Colonic Microbiota in vitro. Microb Ecol Health Dis 15: 137-144.

34. Maathuis A, Hoffman A, Evans A, Sanders L, Venema K (2009) The effect of the undigested fraction of maize products on the activity and composition of the microbiota determined in a dynamic in vitro model of the human proximal large intestine. J Am Coll Nutr 28: 657-666.

35. Ladirat SE, Schuren FH, Schoterman MH, Nauta A, Gruppen H, et al. (2014) Impact of galacto-oligosaccharides on the gut microbiota composition and metabolic activity upon antibiotic treatment during in vitro fermentation. FEMS Microbiol Ecol 87: 41-51.

36. Van der Kamp JW, Jones J, Mccleary B, Topping DJ (2010) Dietary Fibre: New Frontiers for Food and Health: Wageningen Academic Publishers.

37. Marino PL, Sutin KM (2012) The ICU Book: Wolters Kluwer Health, Philadelphia, USA.

38. DeSantis TZ, Hugenholtz P, Larsen N, Rojas M, Brodie EL, et al. (2006) Greengenes, a chimera-checked 16S rRNA gene database and workbench compatible with ARB. Appl Environ Microbiol 72: 5069-5072.

39. Cole JR, Wang Q, Fish JA, Chai B, McGarrell DM, et al. (2014) Ribosomal Database Project: data and tools for high throughput rRNA analysis. Nucleic Acids Res 42: D633-642.

40. Martens EC, Chiang HC, Gordon JI (2008) Mucosal glycan foraging enhances fitness and transmission of a saccharolytic human gut bacterial symbiont. Cell Host Microbe 4: 447-457. 
41. Turroni F, Bottacini F, Foroni E, Mulder I, Kim JH, et al. (2010) Genome analysis of Bifidobacterium bifidum PRL2010 reveals metabolic pathways for hostderived glycan foraging. Proc Natl Acad Sci U S A 107: 19514-19519.

42. Sonnenburg JL, Xu J, Leip DD, Chen CH, Westover BP, et al. (2005) Glycan foraging in vivo by an intestine-adapted bacterial symbiont. Science 307: 1955-1959.

43. Hoskins LC (1993) Mucin degradation in the human gastrointestinal tract and its significance to enteric microbial ecology. Eur J Gastroenterol 5: 205-213.

44. Hoskins L, Boulding E, Gerken T, Harouny V, Kriaris M (1992) Mucin glycoprotein degradation by mucin oligosaccharide-degrading strains of human faecal bacteria. Characterisation of saccharide cleavage products and their potential role in nutritional support of larger faecal bacterial populations. Microb Ecol Health Dis 5: 193-207.

45. Pultz NJ, Hoskins LC, Donskey CJ (2006) Vancomycin-resistant Enterococci may obtain nutritional support by scavenging carbohydrate fragments generated during mucin degradation by the anaerobic microbiota of the colon. Microb Drug Resist 12: 63-67.

46. Cummings JH, Englyst HN (1987) Fermentation in the human large intestine and the available substrates. Am J Clin Nutr 45: 1243-1255.

47. Macfarlane G, and J.H. Cummings (1999) The large intestine: physiology, pathophysiology, and disease. In: Phillips SF PJ, Shorter RG, editor. The colonic flora, fermentation, and large bowel digestive function. New York: Raven Press.

48. Kashyap PC, Marcobal A, Ursell LK, Smits SA, Sonnenburg ED, et al. (2013) Genetically dictated change in host mucus carbohydrate landscape exerts a diet-dependent effect on the gut microbiota. PNAS 110: 17059-17064.

49. Wintermute EH, Silver PA (2010) Emergent cooperation in microbial metabolism. Mol Syst Biol 6: 407.

50. Derrien M, van Passel MWJ, van de Bovenkamp JHB, Schipper RG, de Vos WM, et al. (2010) Mucin-bacterial interactions in the human oral cavity and digestive tract. Gut Microbes 1: 254-268.

51. Png CW, Linden SK, Gilshenan KS, Zoetendal EG, McSweeney CS, et al. (2010) Mucolytic bacteria with increased prevalence in IBD mucosa augment in vitro utilization of mucin by other bacteria. Am J Gastroenterol 105: 2420-2428.

52. Samuel BS, Gordon JI (2006) A humanized gnotobiotic mouse model of hostarchaeal-bacterial mutualism. Proc Natl Acad Sci U S A 103: 10011-10016.

53. Van den Abbeele P, Van de Wiele T, Verstraete W, Possemiers S (2011) The host selects mucosal and luminal associations of coevolved gut microorganisms: a novel concept. FEMS Microbiol Rev 35: 681-704.

54. Belzer C, de Vos WM (2012) Microbes inside from diversity to function: the case of Akkermansia. ISME J 6: 1449-1458. 
55. Tran TH, Boudry C, Everaert N, Thewis A, Portetelle D, et al. (2016) Adding mucins to an in vitro batch fermentation model of the large intestine induces changes in microbial population isolated from porcine feces depending on the substrate. FEMS Microbiol Ecol 92.

56. Willis CL, Cummings JH, Neale G, Gibson GR (1996) In Vitro Effects of Mucin Fermentation on the Growth of Human Colonic Sulphate-Reducing Bacteria: Ecology. Anaerobe 2: 117-122.

57. Yen S, McDonald JAK, Schroeter K, Oliphant K, Sokolenko S, et al. (2015) Metabolomic Analysis of Human Fecal Microbiota: A Comparison of FecesDerived Communities and Defined Mixed Communities. J Proteome Res 14: 1472-1482.

58. Aguirre M, Jonkers DM, Troost FJ, Roeselers G, Venema K (2014) In vitro characterization of the impact of different substrates on metabolite production, energy extraction and composition of gut microbiota from lean and obese subjects. PLoS One 9: e113864.

59. Fakhry S, Manzo N, D'Apuzzo E, Pietrini L, Sorrentini I, et al. (2009) Characterization of intestinal bacteria tightly bound to the human ileal epithelium. Res Microbiol 160: 817-823.

60. Schell MA, Karmirantzou M, Snel B, Vilanova D, Berger B, et al. (2002) The genome sequence of Bifidobacterium longum reflects its adaptation to the human gastrointestinal tract. Proc Natl Acad Sci U S A 99: 14422-14427.

61. Xu J, Bjursell MK, Himrod J, Deng S, Carmichael LK, et al. (2003) A genomic view of the human-Bacteroides thetaiotaomicron symbiosis. Science 299: 2074-2076.

62. Macfarlane S, McBain AJ, Macfarlane GT (1997) Consequences of biofilm and sessile growth in the large intestine. Adv Dent Res 11: 59-68.

63. Macfarlane GT, Cummings JH, Allison C (1986) Protein degradation by human intestinal bacteria. J Gen Microbiol 132: 1647-1656.

64. Chang DE, Smalley DJ, Tucker DL, Leatham MP, Norris WE, et al. (2004) Carbon nutrition of Escherichia coli in the mouse intestine. Proc Natl Acad Sci U S A 101: 7427-7432.

65. Huang JY, Lee SM, Mazmanian SK (2011) The human commensal Bacteroides fragilis binds intestinal mucin. Anaerobe 17: 137-141.

66. Sonnenburg JL, Chen CT, Gordon JI (2006) Genomic and metabolic studies of the impact of probiotics on a model gut symbiont and host. PLoS Biol 4: e413.

67. Duncan SH, Louis P, Flint HJ (2007) Cultivable bacterial diversity from the human colon. Lett Appl Microbiol 44: 343-350.

68. Vince AJ, McNeil NI, Wager JD, Wrong OM (1990) The effect of lactulose, pectin, arabinogalactan and cellulose on the production of organic acids and metabolism of ammonia by intestinal bacteria in a faecal incubation system. $\mathrm{Br}$ J Nutr 63: 17-26. 
69. Wong JM, de Souza R, Kendall CW, Emam A, Jenkins DJ (2006) Colonic health: fermentation and short chain fatty acids. J Clin Gastroenterol 40: 235-243.

70. Burger-van Paassen N, Vincent A, Puiman PJ, van der Sluis M, Bouma J, et al. (2009) The regulation of intestinal mucin MUC2 expression by short-chain fatty acids: implications for epithelial protection. Biochem J 420: 211-219.

71. Van den Abbeele P, Belzer C, Goossens M, Kleerebezem M, De Vos WM, et al. (2013) Butyrate-producing Clostridium cluster XIVa species specifically colonize mucins in an in vitro gut model. The ISME journal 7: 949-961.

72. Doré J, Blottiére H (2015) The influence of diet on the gut microbiota and its consequences for health. Current Opinion in Biotechnology 32: 195-199.

73. Suau A, Rochet V, Sghir A, Gramet Gv, Brewaeys Sp, et al. (2001) Fusobacterium prausnitzii and Related Species Represent a Dominant Group Within the Human Fecal Flora. Syst Appl Microbiol 24: 139-145.

74. Remely M, Tesar I, Hippe B, Gnauer S, Rust P, et al. (2015) Gut microbiota composition correlates with changes in body fat content due to weight loss. Benef Microbes 6: 431-439.

75. Sadaghian Sadabad M, von Martels JZ, Khan MT, Blokzijl T, Paglia G, et al. (2015) A simple coculture system shows mutualism between anaerobic faecalibacteria and epithelial Caco-2 cells. Sci Rep 5: 17906.

76. Tims S, Derom C, Jonkers DM, Vlietinck R, Saris WH, et al. (2013) Microbiota conservation and BMI signatures in adult monozygotic twins. ISME J 7: 707717.

77. Derrien M, Belzer C, de Vos WM (2016) Akkermansia muciniphila and its role in regulating host functions. Microb Pathog.

78. Swidsinski A, Loening-Baucke V, Herber A (2009) Mucosal flora in Crohn's disease and ulcerative colitis - an overview. J Physiol Pharmacol 60 Suppl 6: 61-71.

79. Derrien M, Vaughan EE, Plugge CM, de Vos WM (2004) Akkermansia muciniphila gen. nov., sp. nov., a human intestinal mucin-degrading bacterium. Int J Syst Evol Microbiol 54: 1469-1476.

80. Leimena MM, Ramiro-Garcia J, Davids M, van den Bogert B, Smidt H, et al. (2013) A comprehensive metatranscriptome analysis pipeline and its validation using human small intestine microbiota datasets. BMC Genomics 14: 1-14.

81. Murray WD, Khan AW, van den Berg L (1982) Clostridium saccharolyticum sp. nov., a Saccharolytic Species from Sewage Sludge. Int J Syst Bacteriol 32: 132-135.

82. de Souza Oliveira RP, Perego P, de Oliveira MN, Converti A (2012) Effect of inulin on the growth and metabolism of a probiotic strain of Lactobacillus rhamnosus in co-culture with Streptococcus thermophilus. LWT - Food Sci Technol 47: 358-363. 
83. Hujanen M, Linko S, Linko YY, Leisola M (2001) Optimisation of media and cultivation conditions for $\mathrm{L}(+)(\mathrm{S})$-lactic acid production by Lactobacillus casei NRRL B-441. Appl Microbiol Biotechnol 56: 126-130.

84. Delorme C (2008) Safety assessment of dairy microorganisms: Streptococcus thermophilus. Int J Food Microbiol 126: 274-277.

85. Zhang X, Vrijenhoek JE, Bonten MJ, Willems RJ, van Schaik W (2011) A genetic element present on megaplasmids allows Enterococcus faecium to use raffinose as carbon source. Environ Microbiol 13: 518-528.

86. Finegold SM, Sutter VL, Mathisen GE, Hentges DJ (1983) CHAPTER 1 - Normal Indigenous Intestinal Flora. Human Intestinal Microflora in Health and Disease: Academic Press. pp. 3-31.

87. Sears CL, Myers LL, Lazenby A, Van Tassell RL (1995) Enterotoxigenic Bacteroides fragilis. Clin Infect Dis 20 Suppl 2: S142-148.

88. Xu J, Mahowald MA, Ley RE, Lozupone CA, Hamady M, et al. (2007) Evolution of symbiotic bacteria in the distal human intestine. PLoS Biol 5: e156.

89. Srutkova D, Spanova A, Spano M, Drab V, Schwarzer M, et al. (2011) Efficiency of PCR-based methods in discriminating Bifidobacterium longum ssp. longum and Bifidobacterium longum ssp. infantis strains of human origin. J Microbiol Methods 87: 10-16.

90. Yoshimura K, Matsui T, Itoh K (2010) Prevention of Escherichia coli O157:H7 infection in gnotobiotic mice associated with Bifidobacterium strains. Antonie Van Leeuwenhoek 97: 107-117.

91. Dubourg G, Lagier JC, Armougom F, Robert C, Audoly G, et al. (2013) High-level colonisation of the human gut by Verrucomicrobia following broad-spectrum antibiotic treatment. Int J Antimicrob Agents 41: 149-155.

92. Toh H, Oshima K, Toyoda A, Ogura Y, Ooka T, et al. (2010) Complete genome sequence of the wild-type commensal Escherichia coli strain SE15, belonging to phylogenetic group B2. J Bacteriol 192: 1165-1166. 


\section{Supplemental material}

Table S1. Bacterial strains included in this study.

\begin{tabular}{|c|c|c|c|c|c|c|c|}
\hline Phyla & Strain & Strain number & $\begin{array}{l}\text { Genome } \\
\text { assembly }\end{array}$ & $\begin{array}{l}\text { Genome } \\
\text { size (bp) }\end{array}$ & $\begin{array}{c}\text { OD }^{*} \\
(600 \mathrm{~nm})\end{array}$ & Medium & Reference \\
\hline \multirow{7}{*}{ Firmicutes } & $\begin{array}{l}\text { Clostridinum } \\
\text { saccharolyticum }\end{array}$ & DSM-2544 & ASM14462v1 & $4,662,871$ & 1.561 & $\begin{array}{l}\text { RCB with } \\
\text { additions }\end{array}$ & [81] \\
\hline & $\begin{array}{l}\text { Faecalibacterium } \\
\text { prausnitzii }\end{array}$ & $\begin{array}{l}\text { A2-165 } \\
\text { (DSM 17677) }\end{array}$ & ASM16201v1 & $3,090,349$ & 1.41 & YCFAG & {$[30]$} \\
\hline & $\begin{array}{l}\text { Lactobacillus } \\
\text { rhamnosus }\end{array}$ & ATCC 8530 & ASM23375v1 & $2,960,339$ & 1.619 & $\begin{array}{l}\text { MRS + } 0.05 \% \\
\text { Cysteine }\end{array}$ & [82] \\
\hline & Lactobacillus casei & ATCC 334 & ASM1452v1 & $2,924,325$ & 1.606 & $\begin{array}{l}\text { MRS + } 0.05 \% \\
\text { Cysteine }\end{array}$ & [83] \\
\hline & Eubacterium rectale & ATCC 33656 & ASM2060v1 & $3,449,685$ & 1.171 & $\begin{array}{l}\text { RCB with } \\
\text { additions }\end{array}$ & [20] \\
\hline & $\begin{array}{l}\text { Streptococcus } \\
\text { salivarius subsp. } \\
\text { thermophilus }\end{array}$ & $\begin{array}{l}\text { ATCC BAA- } \\
250\end{array}$ & ASM1182v1 & $1,796,846$ & 1.208 & $\begin{array}{l}\text { M17 with } 0.5 \% \\
\text { glucose }\end{array}$ & [84] \\
\hline & Enterococcus faecium & $\begin{array}{l}\text { ATCC BAA- } \\
472\end{array}$ & ASM17439v2 & $3,052,572$ & 0.937 & $\begin{array}{l}\text { MRS + } 0.05 \% \\
\text { Cysteine }\end{array}$ & [85] \\
\hline \multirow{3}{*}{ Bacteroidetes } & $\begin{array}{l}\text { Bacteroides } \\
\text { thetaiotaomicron }\end{array}$ & DSM-2079 & ASM1106v1 & $6,293,399$ & 1.590 & $\begin{array}{l}\text { RCB with } \\
\text { additions }\end{array}$ & [86] \\
\hline & Bacteroides fragilis & DSM-2151 & ASM2598v1 & $5,241,700$ & 1.809 & $\begin{array}{l}\text { Chopped Meat } \\
\text { Broth with } \\
\text { additions }\end{array}$ & [87] \\
\hline & Bacteroides vulgatus & DSM-1447 & ASM1282v1 & $5,163,189$ & 1.800 & $\begin{array}{l}\text { Chopped Meat } \\
\text { Broth with } \\
\text { additions }\end{array}$ & [88] \\
\hline \multirow{2}{*}{ Actinobacteria } & $\begin{array}{l}\text { Bifidobacterium } \\
\text { longum subsp. infantis }\end{array}$ & DSM-20088 & ASM2042v1 & $2,832,748$ & 1.819 & $\begin{array}{l}\text { RCB with } \\
\text { additions }\end{array}$ & [89] \\
\hline & $\begin{array}{l}\text { Bifidobacterium } \\
\text { adolescentis }\end{array}$ & DSM-20083 & ASM70286v1 & $2,051,152$ & 1.900 & $\begin{array}{l}\text { RCB with } \\
\text { additions }\end{array}$ & [90] \\
\hline Verrucomicrobia & $\begin{array}{l}\text { Akkermansia } \\
\text { muciniphila }\end{array}$ & DSM-22959 & ASM2022v1 & $2,664,102$ & 1.473 & $\begin{array}{l}\text { Chopped Meat } \\
\text { Broth with } \\
\text { additions }\end{array}$ & [91] \\
\hline Proteobacteria & Escherichia coli & SE15 & ASM1048v1 & $4,839,683$ & 0.675 & TS & [92] \\
\hline
\end{tabular}

*OD = optical density at which cultures were harvested.

Table S2. Summary of Differentially Expressed Probes t72.

\begin{tabular}{|c|c|} 
Community & $\begin{array}{c}\text { \# Muc- Control } \\
\text { Diff. } \\
\text { Expr. Probes } \\
\text { p-ttest }<\mathbf{0 . 0 1}\end{array}$ \\
\hline DC & 2300 \\
\hline Lean & 4414 \\
\hline Obese & 1457 \\
\hline DC or Lean or Obese & 7968 \\
\hline DC and Lean and Obese & 3 \\
\hline DC and Lean & 76 \\
\hline DC and Obese & 19 \\
\hline Lean and Obese & 111 \\
\hline
\end{tabular}




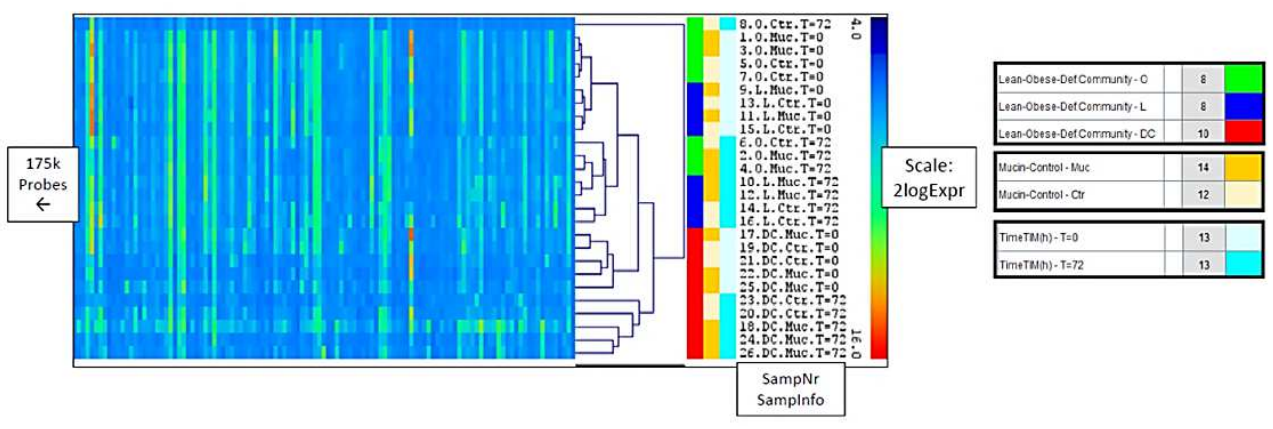

Fig. S1. Hierarchical clustering showing a difference among the Obese/Lean/DC samples and time effect.

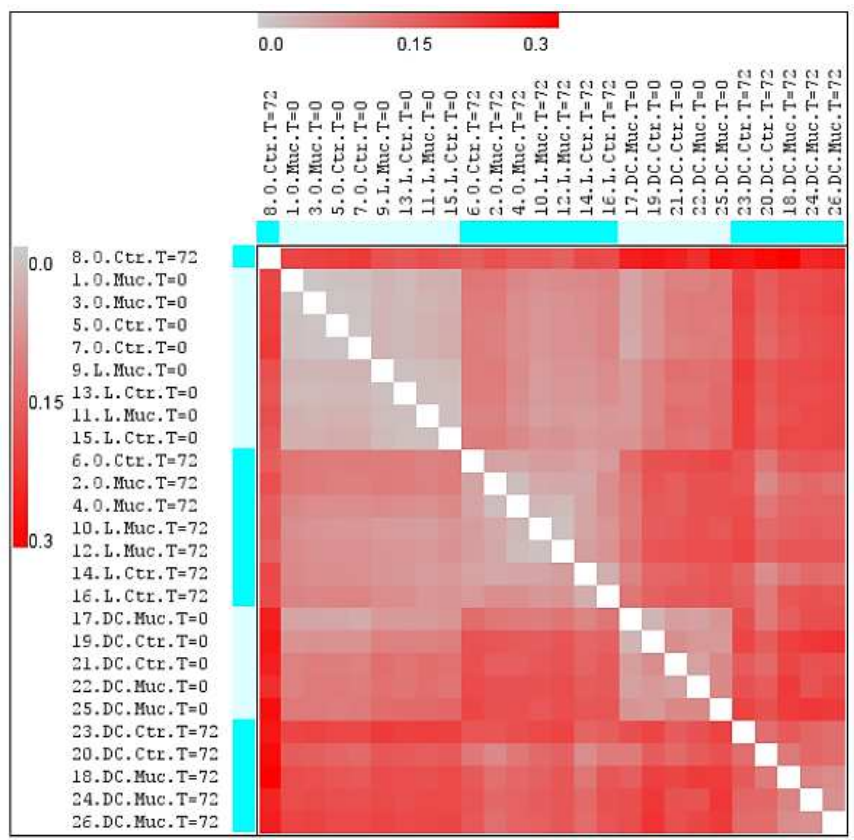

Fig. S2. Correlation analysis. Matrix calculated from 26 conditions (total of samples analyzed in this batch). When the correlation index is close to 0 samples were very similar, when it is 1 they were very different. 

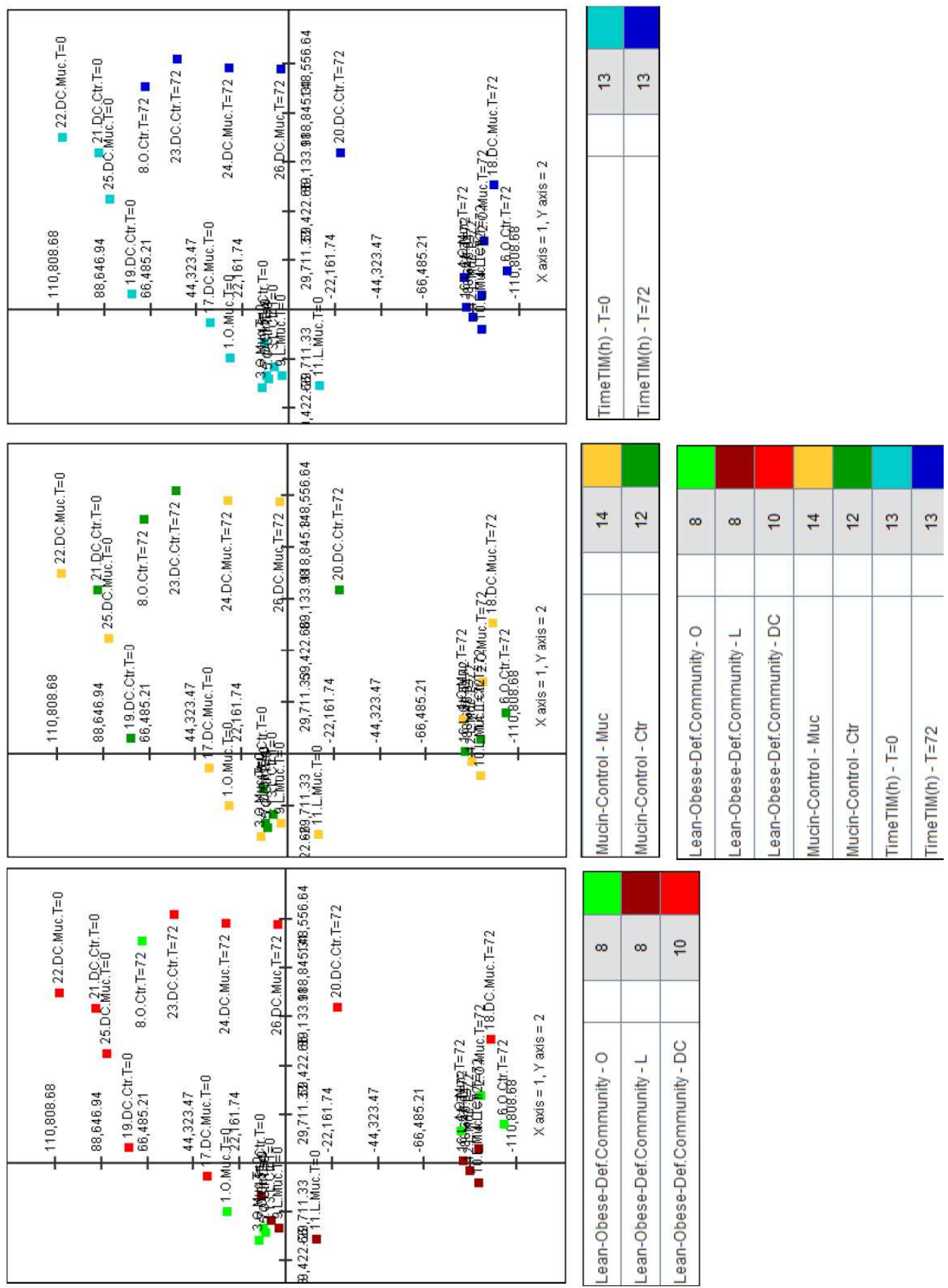

Fig. S3. PCA analysis PC1 $28.14 \%$ of all variation and PC2 $20.14 \%$ of variation). 


\section{Chapter 9}

\section{General Discussion}

Partly published as:

Aguirre M, Venema K (2015) The use of fecal samples for studying human

obesity.

Eur J Epidemiol 30(9):1067-9.

Aguirre M, Venema K. Challenges in simulating the human gut for understanding the role of the microbiota in obesity

Submitted 


\section{Introduction}

The research described in this $\mathrm{PhD}$ thesis was part of a large project embedded in the Gastrointestinal Health theme from the Top Institute of Food \& Nutrition (TIFN). The project was entitled "Molecular interactions of mucosal tissues, bacteria and fibers". The aim was to understand the mechanisms involved in the interactions between diet (fibers), intestinal bacteria and intestinal mucosa function. As part of this project, the research in this thesis aimed to investigate obesity considering three important factors: microbiota, energy balance and metabolism. The approach used was the in vitro fermentation of fibers with microbiota from lean or obese subjects in the TIM-2 system combined with powerful molecular analytical techniques. Hence, the differences in the fermentation of fibers by lean and obese microbiota could be monitored. The knowledge gained provides a basis for further research, which should be focused on targeted dietary interventions to improve human health via de modulation of gut microbiota.

In this last chapter the main findings of this thesis are discussed and recommendations for further research are addressed in each section of the discussion. Last but not least, a conclusion is presented at the end of this chapter.

\section{Methodological considerations and challenges}

\section{Challenges in reproducing the community from the human intestine: from guts to facts}

Most studies evaluating the impact of the intestinal microbiota and its metabolic products on host health have been conducted in animal models, human feces, in vitro batch or continuous culture systems simulating the human gastrointestinal tract.

Determining the fermentation of indigestible compounds by the colonic microbiota is relatively easy in in vitro systems simulating the human gastrointestinal tract. Earlier studies indicate that the use of these tools offers a rapid approach for the estimation of the energy input from specific substrates to the host. Furthermore, these techniques are faster, cheaper and ethically superior than in vivo studies. Fecal samples are used to conduct research in this area. The analysis of feces allows the estimation of a wide catalogue of information from individuals, their unique genetic fingerprinting, pathogens and food habits. Furthermore, numerous metabolites can be analyzed from fecal material. Non-invasive molecular 
techniques increase the resolution of the analysis of feces. Therefore, considering fecal matter as waste is called into question.

In our letter to the Editor of the European Journal of Epidemiology [1] we raised these facts in reply to an opinion from Raoult \& Henrissat [2] who considered the analysis of feces as an indirect and non-optimal method to study the role that microbiota plays in obesity. Raoult \& Henrissat [2] suggest, instead, to study the small intestine to overcome the bias that studying feces implies. However, the community from the small intestine is much simpler compared to the colonic microbiota with respect to diversity, number of different species present, metabolic activity and microbial density. In contrast, the fecal microbiota has been considered representative to the luminal microbiota of the colon [3].

Though interesting, the suggestion of studying the microbiota in the small intestine also carries the inconvenience of the availability of samples and the high invasive procedures to obtain them. The small intestine in healthy individuals is poorly accesible and volunteers from whom samples are taken via intraluminal naso-ileal catheters are fasted and they often require luminal flushing which represents a bias for the analysis of a representative community [4]. We, however, also acknowledge the limitations of using feces for studying human obesity. In some circumstances, the use of fresh human microbiota is not possible because donors live far away from the laboratory or because they are not continuously available to repeatedly participate at various times during the study. In addition, the use of feces for fermentation studies requires the strict anaerobic handling of the samples, although this is also the case for samples from the small intestine. However, collecting feces is a fast and harmless method which could be standardized. Therefore, we argue that the analysis of feces supported by the revolutionary molecular techniques available can incorporate (non-invasively) a complete picture of which microbes play a crucial role in our health and how, metabolically speaking, they impact our lives, also in obesity.

\section{Subject selection}

A challenge in in vitro fermentation experiments has been the improvement of the current standard operational procedures from in vitro systems in order to guarantee the representative, reproducible and stable growth of bacterial communities from fecal samples [5]. For instance, involving participants from different backgrounds (including gender, diet, geographic location, ethnicity, age and antibiotics-use) is one of the factors that influence the sometimes contradictory results in gut microbiota research [6].

Given the inter-individual diversity in the composition of the human gut microbiota and the convenience of having a more representative stock of microbiota of the 
general population, the inclusion of different subjects sharing a wide range of characteristics (i.e. age, diet, geographic location) for the preparation of the inocula for in vitro fermentation studies has been performed [7-11]. However, the fermentation profiles resulting from using a pool of microbiota have been considered as merely the reflection of competitive exclusion (niche exclusion principle) among the bacteria [12] and, therefore, not physiologically relevant. In this scenario, the competition of the most dominant species from one or a certain number of donors is reflected. Based on this, this has been considered an unnatural ecosystem [12].

Yet, some studies provide indication that a pool of microbiota does not present an abnormal metabolic activity or bacteria distribution. The fermentation patterns of arabinoxylan and inulin tested using a pooled inocula (TIM-2) vs. a single volunteer (SHIME), unfolded similar changes in the composition and activity of both types of bacterial communities [13]. Though the direct comparison between the single $v s$. pooled microbiota was not the scope of the study, the results stressed the enormous functional overlap within the microbiota of different individuals, probably as a result of metabolic cross-feeding [14,15]. Furthermore, the cultivation of feces from three different donors and a pooled sample of these subjects in minibioreactor arrays (MBRAs) also showed that despite the differences in the developed communities, the pooled fecal sample seemed to be an average of the composition and structure of the communities from the different donors. BrayCurtis and Sorrenson analyses for dissimilarity in shared OTUs to total OTUs showed in this study that, although the composition and structure of the donors represented the characteristic uniqueness of an individual's gut microbial community by forming separate clusters, the data from the pool indicated that this microbiota included a large proportion of the community members from the donors [16].

In order to have a proper estimation about the impact of preparing an inoculum either from a single donor or a pool of donors, the composition as well as the activity of the microbiota in these two different preparations was investigated in TIM-2 (chapter 2). To the best of our knowledge this was the first time that this direct comparison has been performed.

Our findings underscored the substantial functional overlap in the microbiota of individuals when compared to a pool prepared from them, despite the differences observed in certain groups of bacteria detected during the fermentations. The results showed that the use of a pool of microbiota for in vitro studies does not result in a bacterial community with an aberrant profile and activity compared to that normally obtained from single donors demonstrating the suitability of the preparation of a pool of fecal sample to be used for fermentation experiments. 
As previously explained by Payne et al. [17], it is possible to test the effects of different compounds on the gut microbiota from different donors. However, as noted by these authors, this implies high costs due to the experimental set up and the use of labor force. Although differences are observed in certain groups of bacteria affecting diversity values, the variation in community richness is slightly larger but more stochastic in pooled samples [18]. The next step to proceed is to determine the number of donors which would be required in the preparation.

\section{Preparation of fecal inocula}

A highly even and highly diverse microbiota translates into functional stability despite its exposure to possible hostile conditions characteristic from the gut to in vitro environment transition. In fact, high initial microbiota evenness has been recommended for good functionality even under non-stressed conditions $[19,20]$. Therefore, a well-defined and stable community from the start of in vitro experiments guarantees a reliable interpretation of the data that are generated [21]. There is a lack of studies investigating the effects on microbiota diversity and fermentative activity caused during the preparation and storage at low temperatures of (standardized) human fecal inocula for in vitro fermentations. Differences in the composition and activity of the community may occur from adding different supplements (such as buffer, glycerol, bile salts) or the way of freezing [22]. Furthermore, the addition of such supplements may also have an effect on the redox potential and osmotic stress in the cells contained in the inoculum [23,24].

Part of the studies investigating the optimal preparation of inocula for in vitro studies have focused on testing equine, canine or pig feces or rumen fluid [25-28]. From such studies drastic effects have been found on bacterial cell membrane and DNA integrity affecting the phylogenetic structure and leading to the decline of diversity in the community as well as alterations in the production of gases and kinetics of fermentation. However, these negative effects were not observed during the preparation of a human fecal inoculum by Rose et al. [29] which, to our knowledge, is one of the few studies that have validated the use of fresh and frozen human microbiota. These authors observed that the viable cells in the microbiota stored for 44 weeks at $-80{ }^{\circ} \mathrm{C}$ were not affected and the microbial diversity of this inoculum did not substantially differ from the fresh one, although in that previous study the direct comparisons between a frozen and fresh inoculum were not performed as the main goal.

Therefore, chapter 3 explored different treatments to prepare a standardized pooled fecal inoculum in order to determine the most efficient alternative similar to a fresh inoculum. Treatments were selected based upon routinely used materials (i.e. glycerol, saline suspension, vitamins, and minerals) and procedures (deep 
freezing in liquid nitrogen and storage at $-80{ }^{\circ} \mathrm{C}$ ) in a lab acquainted with preparing long-term stocks of fecal suspensions. The use of a saline preparation is common in the homogenization of slurry for fecal transplants [30,31]. Furthermore, for technical practicalities of working with stocks of gut microbiota, dialysate (a composite previously described Maathuis et al. [32]) was selected to prevent osmotic shock/stress of the cells. Glycerol has also been recommended when creating stocks of bacterial cultures [24] as a cryoprotectant capable of preserving the viability of cells [33]. And importantly, a freezing regime in liquid nitrogen has shown to provide a maximum recovery of cells [34].

In this study, it was demonstrated that it is possible to preserve the characteristic phylogenetic groups in the human gut microbiota, as well as the metabolic activity, both closely resembling fresh feces.

Investigating the optimal preparation and storage of an inoculum for fermentation studies is of high relevance since this may also allow the creation of a large batch of inocula, which can be used for many different experiments, assuring reproducibility and allowing comparison between experiments. Further experiments are recommended to i) test the optimal time and alternatives for thawing as e.g., discussed by Hamilton et al. [31] who tested thawing using an icebath, ii) study the extent of the effects of preparing a human fecal inoculum on in vitro experiments fermenting specific substrates, and iii) determine the effect of different treatments on microbial enzyme activities and gas production.

\section{The in vitro gut simulation}

The implementation of reproducible, accurate and biologically significant in vitro modeling of the human gut is a real need to unravel the role of the gut microbiota in human health. Despite that many of the current in vitro models simulating the human gut use their specific standard protocols, further research is needed in order to reach consistency of the results obtained from these models. Important to ensure is the repeatability, robustness and reproducibility of the model since these are crucial for i) making comparisons among several experiments in which it is vital to have similar starting communities [35] and ii) attributing the effects on the microbiota composition/activity due to the applied treatment and not due to the adaptation of this microbiota to the artificial environment [17,35]. Furthermore, the model has to be capable of maintaining the high diversity [5] and functional complexity of the microbiota remaining representative to the human gut. In chapter 4 we characterized compositionally (16S rRNA sequencing) and functionally (metabolite quantification and metatranscriptomics sequencing) the microbial community developed in TIM-2 in a $72 \mathrm{~h}$ fermentation of a normal diet, a diet high in carbohydrates or a diet high in protein. Our results corroborated that 
the gut microbiota preferably ferments carbohydrates but switches to protein fermentation in case of carbohydrate depletion. This observation is consistent with previous reports [36,37]. Furthermore, our study highlighted the sensitivity and the potential of the TIM-2 system as a tool for investigating the role of the microbiota on human health or disease through investigating reproducible changes in community structure and activity, statistically demonstrated and reflected even at the gene expression level. This allows for comparison of multiple different experiments and even potentially the creation of a large database of substratemicrobiota interactions, which may allow for correlations between certain dietary substrates and microbiota changes. E.g., deciphering structure-function relationships of fermentative fibers and prebiotics may be possible, as this requires a highly reproducible starting microbiota and robust conditions.

\section{Impact of different substrates on the fermentation by lean and obese microbiota}

Due to the effects on satiety and colon health, the impact of carbohydrate and protein fermentation on the gut environment from obese subjects warrants further research. First, the fermentation of carbohydrates may lead to a reduction of food intake, development of fat mass and body weight [38]. Therefore, fermentable carbohydrates may constitute an attractive tool to decrease the incidence of obesity. Second, the consumption of proteins has been proposed as an effective strategy for losing weight in overweight or obese subjects [39]. Protein has been found to have more than 3 times the thermic effect of carbohydrates or fat [40] and provides a higher satiety feeling when compared to the other macronutrients $[40,41]$ with a lower contribution to entire energy requirements (10-30\%) [42]. However, the effect of putrefactive protein fermentation by the microbiota has to be further studied, in order to allow safe use of protein consumption in treating obesity. The breakdown of peptides and proteins do contribute to the formation of short chain fatty acids (SCFA) as seen in vivo and in vitro [43]. However, the characteristic products from proteolytic fermentation: branched chain fatty acids (BCFA; principally iso-butyric, iso-valeric), phenolics (phenol, indole, $p$-cresol, skatole) and ammonia are considered detrimental to the host. A wide variety of negative effects, such as increase of DNA synthesis, disturbances in morphology and intermediate metabolism of colonocytes, have been suggested to be caused by these metabolites $[44,45]$. Therefore, an increase in dietary carbohydrate to decrease the concentration of proteolytic metabolites has been suggested in order to counteract such effects [46]. Studies evidence that shifts in carbohydrate:protein ratio alters the products from the fermentation. However, the influence of such shifts on the 
composition of the microbiota is still unclear. Furthermore, there is no consensus about how fast and reproducibly human gut microbiota can respond to short-term changes in the diet and there is scarce available information addressing this question. One of the few studies was performed by David et al. [47] who elegantly demonstrated that the microbiota can rapidly respond during the exposure of five consecutive days to an altered diet and how this triggered changes in microbial gene expression.

Once all the methodological challenges discussed in chapters 2-4 were evaluated and improved, we further proceeded with assessing the diet-microbe interaction in TIM-2. In chapter 5 we evaluated the potential use of the TIM-2 model as a tool to follow effects of different diets on production of metabolites and microbiota composition. In this study, we investigated the response of gut microbiota obtained from healthy individuals to specific diet changes: high carbohydrate diet $v s$. high protein diet. Different bacterial communities increased or decreased during the feeding of the diets. Such effect was accompanied by a variation in the SCFA and BCFA production. Importantly, the response of the microbiota reflected a trade-off between saccharolytic and proteolytic fermentation. This study brought evidence about the impact of diet on the gut microbiota and the plasticity of this microbiota which effects can be rapidly observed after $24 \mathrm{~h}$ of exposing the microbiota to different proportions of dietary compounds. Furthermore, we concluded that the TIM-2 system provides a high resolution view on the modulatory effects of diet on the gut microbiota.

After corroborating the suitability of the TIM-2 to investigate the network connecting diet, microbiota and metabolites, we focused our efforts on exploring the differences in the fermentation profiles between lean and obese microbiota.

Dietary loads from a Western diet are believed to confer an evolutionary pressure on the gut microbiota [48]. This evolutionary pressure forces the community to adapt to high energy loads derived from highly processed foods and are believed to subsequently determine microbial niches [48] and possibly confer an increased metabolic activity. When studying obesity, increased microbial activity can also be translated into an increased energy harvest from diet which has an impact on host energy metabolism $[48,49]$. The mechanism behind the redefined microbial niches, mediated by more abundant or even new species, urgently requires: first, i) to reach a consensus about the definition of a healthy or normal microbiota (which is not available yet) and ii) more studies focusing on the modulatory effect of the diet on the microbiota $[48,50]$.

As described in chapter 1, there has been a limited number of reports (in vitro) dealing with the differences of lean and obese microbiota. In regard to dietary fibers, their consumption has been found to have a direct impact on the microbial 
fermentation capacity [51]. In this respect, studies suggest that the gut microbiota from obese subjects could be more efficient in extracting energy from diet than lean subjects [52-54]. However, in chapter 6 and 7 we proposed that fermentation of fibers by lean or obese microbiota, and consequently their health effects, may be substrate dependent.

The studies in chapter $\mathbf{6}$ and $\mathbf{7}$ add to knowledge by suggesting that not all substrates are fermented in an identical manner by the gut microbiota, as clearly shown by the measurements of SCFA and BCFA, and the different abundance shifts observed in both microbiotas. Substrate dependency could be explained by the fact that the different dietary fibres tested reflect a heterogeneous group of compounds that differ in their chemical structure and physico-chemical properties, therefore acting on different physiological functions or health benefits $[55,56]$. Additionally, the results point to the possible implications in energy extraction if similar effects happen in vivo. The new information provided on substrate differences between lean and obese subjects is important for researchers to understand if dietary intervention is of help to reduce obesity. However, interindividual differences have to be considered for a full assessment of the impact of certain dietary compounds on the microbiota. Therefore, future studies including in vivo trials are suggested to explore the similarities in the response of diet in different individuals.

\section{Last but not least: mucins}

Planktonic, or luminal populations, constitute the biggest part of the complex microbiota found in the large intestine [57]. Most of the information regarding colonic microbiota has been derived from planktonic bacteria typically found in feces [57]. However, (sessile) bacteria forming biofilms in the mucus layer of the gut intestine has also been suggested to play an important role in human health [58].

The microenvironment created by the mucus layer exists in an equilibrium state between the bacteria growing from the mucus gel and the shedding of epithelial cells and mucus into the lumen [57]. Some bacteria have the ability to grow on colonic mucus and mucin from the upper gastrointestinal tract (gastric, biliary, bronchial and small intestinal) which are used as sources of carbon, nitrogen and energy [59-65]. For instance, intestinal bacteria such as Bacteroides, Bifidobacterium and Akkermansia can use the mucus layer as a source of nutrients $[66,67]$. However, determining the exact composition of mucin-degrading bacteria from the human gut is limited due to i) difficulties in sampling and compositional differences of the studied mucosal samples (rectal swabs, mucus, biopsies), and ii) characteristic inter-individual variability at the taxonomic level of the bacteria [68]. 
Despite this, studies often refer to Bacteroidetes, Actinobacteria and Verrucomicrobium as phyla groups presenting enriched mucosa-associated bacteria [69].

Integrating mucin as a carbon source in an in vitro system simulating the human gut increases the ecological significance of the model since it avoids the washout of adherent microbes (e.g. lactobacilli) [70]. Basically, these species do form part of a normal gut community and their growth may be stimulated, which contributes to a more diverse and stable gut community [65]. Therefore, in chapter 8 we described a study performed in order to elucidate the impact of adding mucin in TIM-2 when growing a defined community (harboring 14-members of the 5 most dominant phyla in the human gut) and a complex community (derived either from lean or obese individuals).

The inclusion of mucin (degrading bacteria) is important in the context of obesity. For instance, evidence indicates that the abundance of A. muciniphila inversely correlates with body weight [71-74]. However, findings are controversial since the abundance of $A$. muciniphila has been found to be high in type 2 diabetic patients [75]. Still, the development of an approach using a mucus colonizer for the treatment or prevention of human obesity has been proposed from findings in which the growth stimulation of A. muciniphila has improved the metabolic profile in mice [76]. Examples integrating modules to determine (in vitro) how bacteria colonize and degrade mucus include: microcosms covered in mucin (in SHIME [70]), insertion of mucin gels in glass tubes [65] and mucin beads encased within a dialysis membrane [77].

Partially purified porcine gastric mucin is used in fermentation studies with gut microbiota aiming to incorporate mucin as a carbon source. Though the origin is different (gastric mucin $v s$. colonic mucin), it has been observed that there is a high similarity (compositionally and structurally) to human colonic mucin [78]. Our results illustrate how some members of the gut microbiota can adapt their metabolic activity to the utilization of mucin. The presence of mucin modified the fermentation in the three different communities in terms of fermentation kinetics, metabolites produced and compositional profiles, which were shifted in the presence of mucin when the communities were compared among each other and against their respective controls. Furthermore, given these effects, the results suggest the possible inclusion of mucin when growing defined communities for synthetic stool transplantations. Yet, our defined community was far from representing the complex communities studied and more efforts are needed in tuning the right compositional balance from different species to closely simulate the human gut ecology. 
General discussion

\section{Concluding remarks}

This thesis raises the awareness that there are differences in the fermentation patterns of fibers in lean and obese microbiota. The metabolites (primarily SCFA) produced and the changes in the composition of the communities observed resulting from the fermentation of the different substrates may play a role in the mechanisms by which dietary components are physiologically beneficial or harmful to the host depending on their microbiota. However, the effects of such fibers should be tested in vivo. The combination of in vitro systems, including cell models, in order to elucidate host-microbe interactions represents a potential tool for these means. The complex and multiple bioactivities observed for SCFA (such as on the one hand contribution to energy load, while on the other being able to induce satiety through induction of gut hormones), shows that we far from understand these host-microbe interactions. Therefore, more efforts are needed for developing and validating in vitro guts for future research in this area. 


\section{References}

1. Aguirre M, Venema K (2015) The use of fecal samples for studying human obesity. Eur J Epidemiol 30: 1067-1069.

2. Raoult D, Henrissat B (2014) Are stool samples suitable for studying the link between gut microbiota and obesity? Eur J Epidemiol 29: 307-309.

3. Dore J, Clement K (2014) Reply to C Matuchansky. Am J Clin Nutr 99: 650-651.

4. El Aidy S, van den Bogert B, Kleerebezem M (2014) The small intestine microbiota, nutritional modulation and relevance for health. Curr Opin Biotechnol 32C: 14-20.

5. McDonald JA, Schroeter K, Fuentes S, Heikamp-Dejong I, Khursigara CM, et al. (2013) Evaluation of microbial community reproducibility, stability and composition in a human distal gut chemostat model. J Microbiol Methods 95: $167-174$.

6. Aguirre M, Venema K (2015) Does the Gut Microbiota Contribute to Obesity? Going beyond the Gut Feeling. Microorganisms 3: 213.

7. Pylkas AM, Juneja LR, Slavin JL (2005) Comparison of different fibers for in vitro production of short chain fatty acids by intestinal microflora. J Med Food $\mathbf{8}$ : 113-116.

8. Rösch C, Venema K, Gruppen H, Schols HA (2015) Characterisation and in vitro fermentation of resistant maltodextrins using human faecal inoculum and analysis of bacterial enzymes present. Bioact Carbohydr Dietary Fibre 6: 4653.

9. Rumpagaporn P, Reuhs BL, Kaur A, Patterson JA, Keshavarzian A, et al. (2015) Structural features of soluble cereal arabinoxylan fibers associated with a slow rate of in vitro fermentation by human fecal microbiota. Carbohydr Polym 130: 191-197.

10. Beeren SR, Christensen CE, Tanaka H, Jensen MG, Donaldson I, et al. (2015) Direct study of fluorescently-labelled barley beta-glucan fate in an in vitro human colon digestion model. Carbohydr Polym 115: 88-92.

11. Cardelle-Cobas A, Olano A, Corzo N, Villamiel M, Collins M, et al. (2012) In vitro fermentation of lactulose-derived oligosaccharides by mixed fecal microbiota. J Agric Food Chem 60: 2024-2032.

12. Roberts KT, Allen-Vercoe E, Williams SA, Graham T, Cui SW (2015) Comparative study of the in vitro fermentative characteristics of fenugreek gum, white bread and bread with fenugreek gum using human faecal microbes. Bioactive Carbohydrates and Dietary Fibre 5: 116-124.

13. Van den Abbeele P, Venema K, Van de Wiele T, Verstraete W, Possemiers S (2013) Different human gut models reveal the distinct fermentation patterns of Arabinoxylan versus inulin. J Agric Food Chem 61: 9819-9827. 
14. Venema K, van Nuenen MH, van den Heuvel EG, Pool W, van der Vossen JM (2003) The effect of lactulose on the composition of the intestinal microbiota and short-chain fatty acid production in human volunteers and a computercontrolled model of the proximal large intestine. Microb Ecol Health Dis 15: 94-105.

15. Kovatcheva-Datchary P, Egert M, Maathuis A, Rajilic-Stojanovic M, de Graaf AA, et al. (2009) Linking phylogenetic identities of bacteria to starch fermentation in an in vitro model of the large intestine by RNA-based stable isotope probing. Environ Microbiol 11: 914-926.

16. Auchtung JM, Robinson CD, Britton RA (2015) Cultivation of stable, reproducible microbial communities from different fecal donors using minibioreactor arrays (MBRAs). Microbiome 3: 42.

17. Payne AN, Zihler A, Chassard C, Lacroix C (2012) Advances and perspectives in in vitro human gut fermentation modeling. Trends Biotechnol 30: 17-25.

18. Aguirre M, Ramiro-Garcia J, Koenen ME, Venema K (2014) To pool or not to pool? Impact of the use of individual and pooled fecal samples for in vitro fermentation studies. J Microbiol Methods 107: 1-7.

19. Wittebolle L, Marzorati M, Clement L, Balloi A, Daffonchio D, et al. (2009) Initial community evenness favours functionality under selective stress. Nature $\mathbf{4 5 8}$ : 623-626.

20. Wilsey BJ, Potvin C (2000) Biodiversity and Ecosystem Functioning: Importance of Species Evenness in an Old Field. Ecology 81: 887-892.

21. Rajilic-Stojanovic M, Maathuis A, Heilig HG, Venema K, de Vos WM, et al. (2010) Evaluating the microbial diversity of an in vitro model of the human large intestine by phylogenetic microarray analysis. Microbiology 156: 32703281 .

22. Kerckhof F-M, Courtens ENP, Geirnaert A, Hoefman S, Ho A, et al. (2014) Optimized Cryopreservation of Mixed Microbial Communities for Conserved Functionality and Diversity. PLoS ONE 9: e99517.

23. Wilson M (2005) Microbial Inhabitants of Humans: Their Ecology and Role in Health and Disease: Cambridge University Press.

24. Hubalek Z (2003) Protectants used in the cryopreservation of microorganisms. Cryobiology 46: 205-229.

25. Bosch G, Wrigglesworth DJ, Cone JW, Pellikaan WF, Hendriks WH (2013) Effects of preservation conditions of canine feces on in vitro gas production kinetics and fermentation end products. J Anim Sci 91: 259-267.

26. Murray J-AMD, McMullin P, Handel I, Hastie PM (2012) The effect of freezing on the fermentative activity of equine faecal inocula for use in an in vitro gas production technique. Anim Feed Sci Technol 178: 175-182. 
27. Prates A, de Oliveira JA, Abecia L, Fondevila M (2010) Effects of preservation procedures of rumen inoculum on in vitro microbial diversity and fermentation. Anim Feed Sci Technol 155: 186-193.

28. Pastorelli G, Faustini M, Attard E (2014) In vitro fermentation of feed ingredients by fresh or frozen pig fecal inocula. Anim Sci J 85: 690-697.

29. Rose DJ, Venema K, Keshavarzian A, Hamaker BR (2010) Starch-entrapped microspheres show a beneficial fermentation profile and decrease in potentially harmful bacteria during in vitro fermentation in faecal microbiota obtained from patients with inflammatory bowel disease. Br J Nutr 103: 15141524.

30. Kleger A, Schnell J, Essig A, Wagner M, Bommer M, et al. (2013) Fecal transplant in refractory Clostridium difficile colitis. Dtsch Arztebl Int 110: 108-115.

31. Hamilton MJ, Weingarden AR, Sadowsky MJ, Khoruts A (2012) Standardized frozen preparation for transplantation of fecal microbiota for recurrent Clostridium difficile infection. Am J Gastroenterol 107: 761-767.

32. Maathuis A, Hoffman A, Evans A, Sanders L, Venema K (2009) The effect of the undigested fraction of maize products on the activity and composition of the microbiota determined in a dynamic in vitro model of the human proximal large intestine. J Am Coll Nutr 28: 657-666.

33. Waite D, Deines P, Taylor M (2013) Quantifying the impact of storage procedures for faecal bacteriotherapy in the critically endangered New Zealand parrot, the kakapo (Strigops habroptilus). Zoo Biol 32: 620-625.

34. Dan M, Richardson J, Miliotis MD, Koornhof HJ (1989) Comparison of preservation media and freezing conditions for storage of specimens of faeces. J Med Microbiol 28: 151-154.

35. Van den Abbeele P, Grootaert C, Marzorati M, Possemiers S, Verstraete W, et al. (2010) Microbial community development in a dynamic gut model is reproducible, colon region specific, and selective for Bacteroidetes and Clostridium cluster IX. Appl Environ Microbiol 76: 5237-5246.

36. Egert M, de Graaf AA, Smidt H, de Vos WM, Venema K (2006) Beyond diversity: functional microbiomics of the human colon. Trends Microbiol 14: 86-91.

37. Ouwehand AC, Derrien M, de Vos W, Tiihonen K, Rautonen N (2005) Prebiotics and other microbial substrates for gut functionality. Curr Opin Biotechnol 16: 212-217.

38. Aguirre M, Venema K (2015) The art of targeting gut microbiota for tackling human obesity. Genes Nutr 10: 472.

39. Cordain L, Eaton SB, Sebastian A, Mann N, Lindeberg S, et al. (2005) Origins and evolution of the Western diet: health implications for the 21 st century. Am J Clin Nutr 81: 341-354.

40. Crovetti R, Porrini M, Santangelo A, Testolin G (1998) The influence of thermic effect of food on satiety. Eur J Clin Nutr 52: 482-488. 
41. Stubbs RJ (1998) Nutrition Society Medal Lecture. Appetite, feeding behaviour and energy balance in human subjects. Proc Nutr Soc 57: 341-356.

42. Krajmalnik-Brown R, Ilhan ZE, Kang DW, DiBaise JK (2012) Effects of gut microbes on nutrient absorption and energy regulation. Nutr Clin Pract 27: 201-214.

43. Macfarlane S, Macfarlane GT (2003) Regulation of short-chain fatty acid production. Proc Nutr Soc 62: 67-72.

44. Davila AM, Blachier F, Gotteland M, Andriamihaja M, Benetti PH, et al. (2013) Intestinal luminal nitrogen metabolism: role of the gut microbiota and consequences for the host. Pharmacol Res 68: 95-107.

45. Windey K, De Preter V, Verbeke K (2012) Relevance of protein fermentation to gut health. Mol Nutr Food Res 56: 184-196.

46. Scott KP, Gratz SW, Sheridan PO, Flint HJ, Duncan SH (2013) The influence of diet on the gut microbiota. Pharmacol Res 69: 52-60.

47. David LA, Maurice CF, Carmody RN, Gootenberg DB, Button JE, et al. (2014) Diet rapidly and reproducibly alters the human gut microbiome. Nature 505: 559-563.

48. Payne AN (2012) Assessment of dietary modulation on the gut microbiota of obese and normal-weight children using a combined in vivo and in vitro systems biology modeling platform: Diss., Eidgenössische Technische Hochschule ETH Zürich, Nr. 20235, 2012.

49. Aguirre M, Jonkers DM, Troost FJ, Roeselers G, Venema K (2014) In vitro characterization of the impact of different substrates on metabolite production, energy extraction and composition of gut microbiota from lean and obese subjects. PLoS One 9: e113864.

50. Aguirre M, Eck A, Koenen ME, Savelkoul PHM, Budding AE, et al. (2016) Diet drives quick changes in the metabolic activity and composition of human gut microbiota in a validated in vitro gut model. Research in Microbiology 167: 114-125.

51. Aguirre M, Venema K (2015) Does the Gut Microbiota Contribute to Obesity? Going beyond the Gut Feeling. Microorganisms 3: 213-235.

52. Backhed F, Ding H, Wang T, Hooper LV, Koh GY, et al. (2004) The gut microbiota as an environmental factor that regulates fat storage. Proc Natl Acad Sci U S A 101: 15718-15723.

53. Turnbaugh PJ, Backhed F, Fulton L, Gordon JI (2008) Diet-induced obesity is linked to marked but reversible alterations in the mouse distal gut microbiome. Cell Host Microbe 3: 213-223.

54. Turnbaugh PJ, Ley RE, Mahowald MA, Magrini V, Mardis ER, et al. (2006) An obesity-associated gut microbiome with increased capacity for energy harvest. Nature 444: 1027-1131. 
55. Wanders AJ, Jonathan MC, van den Borne JJ, Mars M, Schols HA, et al. (2013) The effects of bulking, viscous and gel-forming dietary fibres on satiation. $\mathrm{Br}$ J Nutr 109: 1330-1337.

56. Cummings JH, Edmond LM, Magee EA (2004) Dietary carbohydrates and health: do we still need the fibre concept? Clin Nutr Suppl 1: 5-17.

57. Probert HM, Gibson GR (2002) Bacterial biofilms in the human gastrointestinal tract. Curr Issues Intest Microbiol 3: 23-27.

58. Croucher SC, Houston AP, Bayliss CE, Turner RJ (1983) Bacterial populations associated with different regions of the human colon wall. Appl Environ Microbiol 45: 1025-1033.

59. Cohen PS, Rossoll R, Cabelli VJ, Yang SL, Laux DC (1983) Relationship between the mouse colonizing ability of a human fecal Escherichia coli strain and its ability to bind a specific mouse colonic mucous gel protein. Infect Immun 40: 62-69.

60. Macfarlane GT, Gibson GR (1991) Formation of glycoprotein degrading enzymes by Bacteroides fragilis. FEMS Microbiology Letters 77: 289-293.

61. Macfarlane S, Hopkins MJ, Macfarlane GT (2001) Toxin synthesis and mucin breakdown are related to swarming phenomenon in Clostridium septicum. Infect Immun 69: 1120-1126.

62. McCormick BA, Stocker BA, Laux DC, Cohen PS (1988) Roles of motility, chemotaxis, and penetration through and growth in intestinal mucus in the ability of an avirulent strain of Salmonella typhimurium to colonize the large intestine of streptomycin-treated mice. Infect Immun 56: 2209-2217.

63. McSweegan E, Walker RI (1986) Identification and characterization of two Campylobacter jejuni adhesins for cellular and mucous substrates. Infect Immun 53: 141-148.

64. Wilson KH, Perini F (1988) Role of competition for nutrients in suppression of Clostridium difficile by the colonic microflora. Infect Immun 56: 2610-2614.

65. Macfarlane S, Woodmansey EJ, Macfarlane GT (2005) Colonization of mucin by human intestinal bacteria and establishment of biofilm communities in a twostage continuous culture system. Appl Environ Microbiol 71: 7483-7492.

66. Hoskins LC, Boulding ET (1981) Mucin Degradation in Human Colon Ecosystems: evidence for the existence and role of bacterial subpopulations producing glycosidases as extracellular enzymes. Journal of Clinical Investigation 67: 163-172.

67. Derrien M, Vaughan EE, Plugge CM, de Vos WM (2004) Akkermansia muciniphila gen. nov., sp. nov., a human intestinal mucin-degrading bacterium. Int J Syst Evol Microbiol 54: 1469-1476.

68. Tailford LE, Crost EH, Kavanaugh D, Juge N (2015) Mucin glycan foraging in the human gut microbiome. Frontiers in genetics 6. 
69. Flint HJ, Scott KP, Duncan SH, Louis P, Forano E (2012) Microbial degradation of complex carbohydrates in the gut. Gut microbes 3: 289-306.

70. Van den Abbeele P, Roos S, Eeckhaut V, MacKenzie DA, Derde M, et al. (2012) Incorporating a mucosal environment in a dynamic gut model results in a more representative colonization by lactobacilli. Microb Biotechnol 5: 106-115.

71. Santacruz A, Collado MC, Garcia-Valdes L, Segura MT, Martin-Lagos JA, et al. (2010) Gut microbiota composition is associated with body weight, weight gain and biochemical parameters in pregnant women. Br J Nutr 104: 83-92.

72. Karlsson CL, Onnerfalt J, Xu J, Molin G, Ahrne S, et al. (2012) The microbiota of the gut in preschool children with normal and excessive body weight. Obesity (Silver Spring) 20: 2257-2261.

73. Collado MC, Isolauri E, Laitinen K, Salminen S (2008) Distinct composition of gut microbiota during pregnancy in overweight and normal-weight women. Am J Clin Nutr 88: 894-899.

74. Everard A, Lazarevic V, Derrien M, Girard M, Muccioli GG, et al. (2011) Responses of gut microbiota and glucose and lipid metabolism to prebiotics in genetic obese and diet-induced leptin-resistant mice. Diabetes 60: 2775-2786.

75. Qin J, Li Y, Cai Z, Li S, Zhu J, et al. (2012) A metagenome-wide association study of gut microbiota in type 2 diabetes. Nature 490: 55-60.

76. Everard A, Belzer C, Geurts L, Ouwerkerk JP, Druart Cl, et al. (2013) Cross-talk between Akkermansia muciniphila and intestinal epithelium controls dietinduced obesity. PNAS 110: 9066-9071.

77. Probert H, Gibson G (2004) Development of a fermentation system to model sessile bacterial populations in the human colon. Biofilms 1: 13-19.

78. Variyam EP, Hoskins LC (1981) Mucin degradation in human colon ecosystems. Degradation of hog gastric mucin by fecal extracts and fecal cultures. Gastroenterology 81: 751-758. 
Summary 
There is an elevated incidence of cases of obesity worldwide. The World Health Organization estimated that by 2014 over 600 million of adults were obese. Furthermore, this health problem also extends to children: 42 million children under the age of 5 were overweight or obese in 2013. For the first time in human history there are more obese people in the world than underweight people. Obese subjects are not only more prone to disease but also suffer from a lower quality of life and they lead to more cost to the health care system. Therefore, the development of strategies to tackle this condition is of vital importance. The potential role of gut microbiota in obesity has recently emerged as a promising therapeutic tool. Therefore, this work aimed to: i) contribute to know what the impact of the gut microbiota is on obesity when fermenting indigestible dietary compounds, and ii) elucidate the plasticity of the microbiota to manipulation and the potential to modify an obese gut phenotype. The literature evidence on the role of the microbiota in obesity is reviewed in Chapter 1.

Chapter 2 focuses on the differences related to the composition and activity of an individual or a pooled preparation of human fecal inoculum during a standard fermentation process. Many in vitro studies generally use a standardized inoculum which is derived from a pool of subjects of interest and is stored frozen. However, the use of an inoculum prepared from either a single donor or a pool of donors remains debatable among experts. The main argument lies in the concern about how representative such inoculum is in regard to the colonic ecosystem taking into account the abundance and the variety of bacterial species. Consequently, the use of an individual or a mixed inoculum is believed to lead to a degree of variation among experiments, even when the single inoculum is repeatedly taken from the same individual over time. With respect to the TIM-2 system, no studies were performed before in order to characterize such possible variations. Therefore, the purpose of this study was to compare both ways of preparing a fecal inoculum to be used in vitro. The results obtained after assessing the suitability of using the two types of inocula showed that the microbiota prepared from a pool of volunteers does not result in an aberrant composition or functional performance. These findings are a keystone for performing an evaluation and improvement of the standardized methods for preparing the inoculum itself.

Chapter 3 investigated an optimal preservation approach to prepare human feces as inoculum for in vitro fermentations as an alternative to the use of fresh feces. Freshly collected fecal samples may not always be available. In some circumstances, the use of fresh human microbiota is not possible because donors live far away from the laboratory or because they are not continuously available to repeatedly participate at various times during the study. To guarantee a constant inoculum over time for different studies, most studies use prepared and stored 
inocula. In these cases, the use of frozen feces provides more flexibility for this type of experiments. However, preparation and storage of fecal samples have been shown to impact microbial composition, viability and activity. It is unknown to what extent various preparation and storage methods impact the microbiota and what preservation method is best. Hence, the effects of different treatments used to prepare human feces as inocula for in vitro fermentation experiments were studied. This study allowed us to propose an easy and optimal preparation for the conservation of feces for in vitro fermentation studies which compositionally and functionally resembled quite closely the golden standard or reference (fresh feces). These findings contribute to increase the reproducibility of the in vitro assays that can be performed over a long period of time with the same microbiota batch, which is impossible with a single fresh fecal sample.

Chapter $\mathbf{2}$ and $\mathbf{3}$ are chapters in this thesis which partially covered the study of different criteria needed to be fulfilled by in vitro gut models before they are considered valid for monitoring the effects of specific interventions/treatments on the microbiota. However, it is equally important to ensure the repeatability, robustness and reproducibility of the model itself. Chapter 4 investigated compositionally and functionally the microbial community developed in TIM-2 in a $72 \mathrm{~h}$ fermentation on a diet high in carbohydrate or high in protein using powerful molecular techniques. This study was performed in order to test if the network connecting diet, microbiota and metabolites production was reproduced in different replicate experiments using the TIM-2 system under the same experimental conditions. Our results indicate the strong reproducibility of the communities developing in TIM-2 at both the level of composition and functionality.

After evaluating the methodological considerations and challenges mentioned in the three chapters above, the following step was to study the effects of diet on the microbiota. Currently, there is no consensus on how fast and reproducibly human gut microbiota can respond to short-term changes in the diet and there is scarce available information addressing this question. Chapter $\mathbf{5}$ presented a screening study in which the effects of a high carbohydrate and a high protein diet on $\mathrm{pH}$, SCFA and BCFA were characterized. This study aimed to provide insight about how fast changes in bacterial activity and composition can be induced in a $72 \mathrm{~h}$ fermentation when major perturbations were performed in the diet supplied to the microbiota.

With chapter 5, knowledge was gained about the impact of diet on the gut microbiota by observing a fast response (24h) from the microbiota to the different diets tested. The sensitivity of the TIM-2 as an in vitro system detecting such impact was also confirmed. Therefore, a comparison was performed between 
fermentation by microbiota from lean and obese subjects. Hence, chapters $\mathbf{6}$ and 7 monitored the fermentation of different prebiotic substrates (galactooligosaccharides, lactulose, apple fiber, sugar beet pectin (chapter 6), arabinogalactan and inulin (chapter 7) by the microbiota from lean or obese subjects. These studies helped to elucidate how different the contribution of energy by the microbiota to the host was during a fermentation process, in terms of metabolites produced. Contrary to expected, the extraction of energy from the obese microbiota differed per substrate and it was not higher in most of the cases when compared to the lean microbiota. Furthermore, the chapters also showed the specific modulation of the composition of the microbiota by these substrates.

Chapter 8 presents a study performed with the aim of: i) gaining insight about how the presence of mucin plays a role in the development of bacterial communities (in vitro), and ii) explore how representative a defined community harboring 14members (comprising the 5 most dominant phyla in the healthy human gut) is in comparison to a complex community (derived either from lean or obese individuals). The majority of current in vitro models do not use the addition of mucin to the culturing media which results only in the simulation of luminal microbiota excluding microorganisms which profit from a mucosal environment to grow optimally. This lack of mucin makes it more challenging in achieving a representative simulation of the human gut compared to the in vivo situation. Although we found that more efforts are needed in achieving a better simulation of a complex microbiota by using a defined community, this chapter contributes with more insight about the growth of defined communities in vitro and evaluating their response to substrates derived from the host. 
Samenvatting 
Wereldwijd komt er een steeds hoger aantal obesitas-gevallen voor. Volgens schattingen van de Wereldgezondheidsorganisatie (WHO) waren er in 2014600 miljoen mensen obees. Bovendien breidt dit probleem zich ook uit naar kinderen: 42 miljoen kinderen onder de leeftijd van 5 jaar waren te zwaar of obees in 2013. Voor het eerst in de menselijke geschiedenis zijn er meer obese mensen dan mensen met ondergewicht op de wereld. Personen met obesitas zijn niet alleen gevoeliger voor ziektes, maar ervaren ook een lagere kwaliteit van leven en ze leiden vaak tot hogere kosten in de gezondheidszorg. Derhalve is de ontwikkeling van strategieën om deze situatie aan te pakken van levensbelang. De rol die het darmmicrobioom speelt bij obesitas is recentelijk aan het licht gekomen als veelbelovende geneeswijze. Daarom had dit werk als doelstelling: i) een bijdrage leveren aan de kennis over de impact van het darmmicrobioom op obesitas bij het fermenteren van onverteerbare bestanddelen van voedingsmiddelen, en ii) meer inzicht geven in de reactie van het microbioom op manipulatie en het potentieel het fenotype van een obese darm te modificeren. Bewijs uit de literatuur betreffende de rol van het microbioom bij obesitas wordt besproken in hoofdstuk 1.

Hoofdstuk 2 concentreert zich op de verschillen gerelateerd aan de samenstelling en activiteit van een individuele of een samengestelde bereiding van een menselijk fecaal inoculum gedurende een gestandaardiseerd fermentatie proces. Over het algemeen gebruiken veel in vitro studies een gestandaardiseerd inoculum dat voortkomt uit een groep interessante personen en dat ingevroren wordt bewaard. Het gebruik van een inoculum van een enkele donor of van een groep donoren blijft onder experts echter een bron van discussie. Het belangrijkste argument betreft de zorg over hoe representatief zo'n inoculum is vergeleken met het ecosysteem van de darm, rekening houdende met de veelheid en de variëteit van de soorten bacteriën. Dientengevolge kan het gebruik van een individueel of een gemengd inoculum leiden tot een mate van variatie in experimenten, zelfs wanneer een individueel inoculum herhaaldelijk wordt genomen van dezelfde persoon gedurende een bepaalde periode. Wat betreft het TIM-2 systeem werd er nog geen enkele studie uitgevoerd om zulke mogelijk variaties in kaart te brengen. Derhalve was het doel van dit onderzoek het vergelijken van beide bereidingswijzen voor een fecaal inoculum voor in vitro gebruik. De verkregen resultaten over de bruikbaarheid van de twee types van inoculum, lieten zien dat het microbioom dat werd bereid uit een groep van vrijwilligers niet resulteert in een afwijkende compositie of werking. Deze bevindingen zijn erg belangrijk voor de uitvoering van een evaluatie en verbetering van de gestandaardiseerde methoden voor de bereiding van het inoculum zelf

In hoofdstuk 3 is onderzoek gedaan naar een optimale conserveringsaanpak voor het bewaren van menselijke feces als inoculum voor in vitro fermentaties, als 
alternatief voor het gebruik van verse feces. Het zou kunnen dat een vers verkregen fecaal monster niet altijd beschikbaar is. Onder bepaalde omstandigheden is het gebruik van een vers menselijk microbioom niet mogelijk, omdat donoren ver van het laboratorium vandaan wonen of omdat ze niet continu beschikbaar zijn om verschillende keren deel te nemen gedurende de studie. Om toch een constant inoculum te kunnen garanderen voor diverse onderzoeken gedurende een periode, gebruiken de meeste onderzoeken voorbereide en opgeslagen inocula. In deze gevallen voorziet het gebruik van ingevroren feces in meer flexibiliteit voor dit type van experimenten. Voorbereiding en opslag van fecale samples hebben echter een effect laten zien op de microbiële samenstelling, levensvatbaarheid en activiteit. Het is onbekend in hoeverre verschillende bereidings- en opslagmethoden het microbioom beïnvloeden en welke conserveringsmethode het beste is. Daarom werden de effecten van verschillende behandelingen, die worden aangewend om menselijke feces, gebruikt als inocula voor in vitro fermentatie, te bereiden, bestudeerd. Deze studie maakte het ons mogelijk een makkelijke en optimale bereiding op te stellen voor feces die gebruikt worden in in vitro fermentatie onderzoeken, welke in samenstelling en functionaliteit erg dicht bij de gouden standaard of referentie (verse feces) komen. Deze bevindingen dragen bij aan het vergroten van de reproduceerbaarheid van in vitro onderzoeken die kunnen worden uitgevoerd gedurende langere tijd met hetzelfde microbioom, hetgeen onmogelijk is met een enkel vers fecaal monster.

Hoofdstuk 2 en 3 zijn hoofdstukken in dit proefschrift die een gedeelte van het onderzoek naar de verschillende criteria beschrijven, waaraan voldaan moet worden door in vitro darmmodellen voordat zij worden beschouwd als representatief voor het monitoren van effecten van specifieke ingrepen aan en behandelingen van het microbioom. Het is echter even belangrijk om de robuustheid en de reproduceerbaarheid van het model zelf te waarborgen. In hoofdstuk 4 is de samenstelling en de functionaliteit onderzocht van de microbiële populatie die zich ontwikkelde in TIM-2 gedurende een 72 uur durende fermentatie op een dieet dat ofwel veel koolhydraten ofwel veel eiwitten bevatte. Hierbij werd gebruik gemaakt van krachtige moleculaire technieken. Dit onderzoek werd uitgevoerd met het doel te testen of het netwerk dat dieet, microbioom en metabolietenproductie met elkaar verbindt, reproduceerbaar was in verschillende, op dezelfde manier opgezette, experimenten, waarbij TIM-2 werd gebruikt onder gelijkblijvende experimentele omstandigheden. Onze resultaten wijzen in de richting van een grote reproduceerbaarheid van de zich in TIM-2 ontwikkelende populaties, zowel op het vlak van samenstelling als op het vlak van functionaliteit.

$\mathrm{Na}$ het evalueren van de methodologische overwegingen en uitdagingen genoemd in de drie voorgaande hoofdstukken, was de volgende stap het bestuderen van de 
effecten van een dieet op het microbioom. Momenteel is er geen concensus over hoe snel en hoe reproduceerbaar het menselijke darm microbioom zich kan aanpassen aan korte termijn veranderingen in het dieet en er is maar weinig informatie te vinden met betrekking tot deze vraag. In hoofdstuk 5 werd een screenings-onderzoek gedaan waarin de effecten werden gekarakteriseerd van een dieet met een hoog gehalte aan koolhydraten en een dieet met een hoog gehalte aan eiwitten op $\mathrm{pH}$, korte-keten vetzuren en vertakte-keten vetzuren. Dit onderzoek had als doel inzicht te verschaffen in hoe snel veranderingen in bacteriële samenstelling en activiteit teweeg kunnen worden gebracht binnen een 72 uur durende fermentatie als er belangrijke veranderingen in het, aan het microbioom verstrekte, dieet worden aangebracht.

In hoofdstuk 5 werd er kennis verzameld over de impact van het dieet op het darmmicrobioom door het waarnemen van een snelle respons van de microbiomen op de verschillende geteste diëten. De gevoeligheid van TIM-2 als in vitro waarnemingssysteem voor zo'n impact werd ook bevestigd. Derhalve werd er een vergelijking gedaan tussen fermentatie door het microbioom van dunne personen en van personen met overgewichtig. Daarvoor werd in hoofdstuk 6 en 7 de fermentatie gemonitord van verschillende prebiotische substraten (galactooligosacchariden, lactulose, appelvezel, suikerbietpectine (hoofdstuk 6) en arabinogalactaan en inuline (hoofdstuk 7) door het microbioom van ofwel dunne personen ofwel personen met overgewicht. Deze onderzoeken hielpen te verduidelijken hoe verschillend de bijdrage van energie door het microbioom aan de gastheer was gedurende het fermentatieproces, kijkend naar de geproduceerde metabolieten. In tegenstelling tot wat werd verwacht, was de extractie van energie door de obese microbiomen verschillend per substraat en niet verhoogd in de meeste gevallen, in vergelijking met het microbioom van dunne personen. Verder lieten de hoofdstukken ook de specifieke modulatie van de samenstelling van de microbiomen door deze substraten zien.

Hoofdstuk 8 presenteert een onderzoek uitgevoerd met als doel: i) inzicht verschaffen in de rol die de aanwezigheid van mucine speelt bij de ontwikkeling van bacteriële populaties (in vitro), en ii) onderzoeken hoe representatief een gedefinieerde populatie van 14-bacteriele species (die de 5 meest dominantie species van een gezonde menselijke darm bevat) is in vergelijking met een complexe populatie (verkregen van ofwel dunne ofwel obese personen). Het grootste gedeelte van huidige in vitro modellen maken geen gebruik van de toevoeging van mucine aan de groeimedia. Dit heeft tot gevolg dat alleen luminale microbiomen gesimuleerd worden en de micro-organismen welke profiteren van een slijmerige omgeving om tot een optimale groei te komen, worden buitengesloten. Dit gebrek aan mucine maakt het moeilijker om tot een 
representatieve simulatie van de menselijke darm te komen in vergelijking tot de in vivo situatie. Hoewel er, volgens onze bevindingen, meer inspanningen nodig zijn om een betere simulatie van een complex microbioom te bewerkstelligen door het gebruik van een gedefinieerde populatie, draagt dit hoofdstuk bij aan meer inzicht in de groei van gedefinieerde populaties in vitro en het evalueren van hun respons op substraten verkregen van de gastheer. 


\section{Valorization}

Aguirre M, Venema K. Challenges in simulating the human gut for understanding the role of the microbiota in obesity 


\section{Social relevance}

According to the World Health Organization (WHO), over 600 million adults around the world were obese in 2014 (Fig. 1) [1]. A $39 \%$ of adults were overweight and around 13\% were obese. Facts also show that obesity is killing more than underweight (!). If this is not disturbingly enough, an increase in obesity is not only seen in adults, but this health problem also extends to children: 42 million children under the age of 5 were overweight or obese in 2013. However, the important message here is that obesity is preventable.

A lot of research has been performed in obesity and associated diseases. Looking at genetics it appears to be the case that the most common gene-environment interactions do not seem to explain the major part of the obesity problem. In the last 10 years the gut microbiota has been increasingly recognized as a contributing factor for obesity. This was the main focus of this project: looking at the dietmicrobe relationship including microbial products like short chain fatty acids (SCFA: acetate, propionate, butyrate) which are produced by the microbiota when they ferment indigestible dietary compounds. These microbial products may have an important impact in our metabolism. On the one hand they may affect signaling of satiety-coupled receptors and on the other hand SCFA may enter the circulation and have more systemic effects (fat accumulation, substrate metabolism in the skeletal muscle, adipose tissue and liver, processes of inflammation, etc.) [2] .

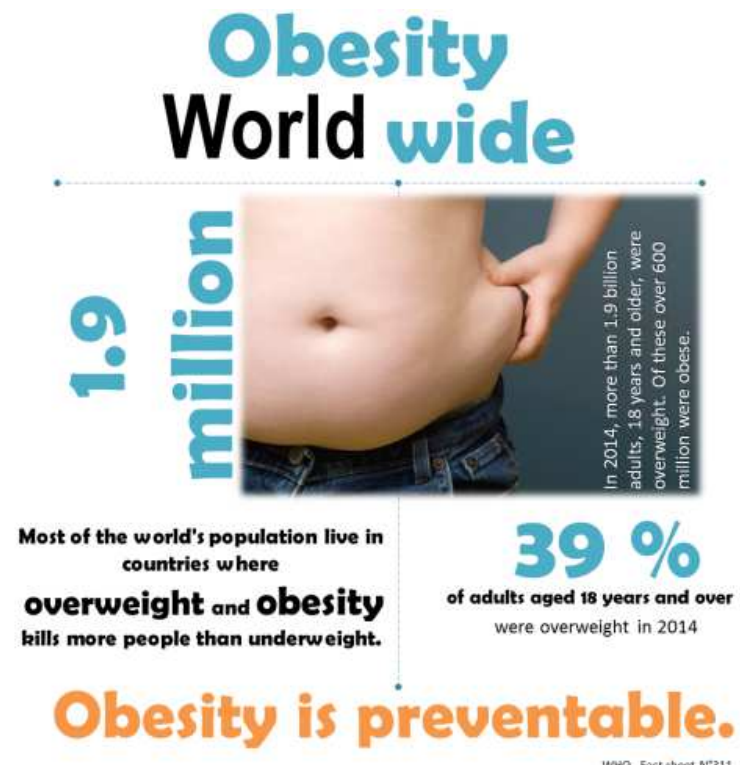

Fig. 1. Key facts about obesity and overweight. 
It is clear that there is an urgent need to tackle obesity, and the possibility of manipulating the gut microbiota as a therapeutic way for either prevention or treatment has taken force in recent years. The scientific outcome of this thesis describing how the fermentation of different dietary compounds occurs in the microbiota from different subjects is one concrete step to elucidate the impact of these communities on host energy balance. This project sets a precedent for future in vivo studies aiming on personalized nutrition research.

\section{Target groups}

Besides the large portion of the population suffering from overweight or obesity specified in the above section, the results from the present study benefit the sector of the food industry focused on prebiotic and probiotic products. Scientific evidence from this project is provided for the use of some prebiotics and how they could modulate the intestinal community as well as for identifying microorganisms which could be key players in a lean profile.

\section{Activities/Products}

The results from the present study could be translated and shaped into:

\section{Bacterial cocktails to be used for treating obesity}

As explained in the introduction chapter, the modification of the gut bacteria could be achieved by administrating different bacteria isolated from the human gut. The concept of having a cocktail of a defined community containing specific bacteria could be used to replace dysfunctional ecosystems with a healthy customized ecosystem [3]. As explained by Petrof et al. [4], the use of a defined community to manipulate the gut microbiota offers a wide range of advantages which include: i) the freedom of selecting specific bacteria for making a defined composition, ii) the capacity for indvidual growth of bacterial species within such defined composition, and iii) better safety profile when compared to complex communities derived from feces. The optimal design and growth of defined communities can be a biological relevant model not only as a new approach to study gut microbial dysbiosis but also for its treatment.

\section{Optimizing in vitro models when studying obesity}

The development of physiological relevant in vitro models for studying the role of the human gut microbiota in obesity could accelerate the development of strategies 
to tackle the elevated incidence of cases of obesity in the worldwide population as well as the potential reduction of animal testing. It is of high importance to optimizae in vitro systems to model human colonic fermentation in obesity. Special attention needs to be focused on: culturing media, the use of a representative control, study of the effect of fats, shear stress, redox potential, anaerobiosis and the simulation of transit time.

\section{Culturing media, when sometimes the proof of the pudding is in the eating}

The main substrates available for microbial fermentation in the large intestine are a mixture of components which, for different reasons, were not (efficiently) digested or absorbed in the small intestine [5,6]. These include dietary oligosaccharides, polysaccharides, peptides and proteins as well as host epithelial cells, mucins and pancreatic secretions $[7,8]$. Members of the gut microbiota preferably ferment carbohydrates [9]. For this reason, the metabolic activity of the residing bacteria in the proximal colon is mostly dedicated to the production of SCFA (with some intermediate metabolites, such as lactate, formate, succinate, acrylate, ethanol) and gases including $\mathrm{H}_{2}, \mathrm{CH}_{4}, \mathrm{CO}_{2}$, which consequently causes a low $\mathrm{pH}(\sim 5.4-5.9)$ in this part of the colon [10]. Due to the depletion of available carbohydrates and the relative increased protein content, the metabolic activity of the bacteria reaching the distal colon switches to proteolytic fermentation with the production of branched-chain fatty acids (BCFA) and toxic compounds including phenols, indoles and ammonia, increasing the $\mathrm{pH}(\sim 6.6-6.9)$ [10].

A culture medium mimicking the terminal ileal chyme from an average Western diet has been widely used in fermentation studies. The composition of this medium has been developed by testing a wide range of different nutritional ratios of polysaccharides (mainly arabinogalactan, pectin, xylan, dextrins and starch), proteins (peptone, caseine), glycoproteins (mucin) and minerals and vitamins against in vivo values of composition and activity (metabolite and gas production, and enzymatic essays) of the microbiota found in samples of the intestinal content of sudden death individuals [11-13]. In the case of batch fermentations, the trophic status can be classified as oligotrophic or eutotrophic as described by Long et al. [14]. Oligotrophic systems are typically inoculated with a high dense fecal slurry (5 to $20 \% \mathrm{w} / \mathrm{v}$ ) which is meant to also provide nutrients, and the media (usually consisting on a phosphate-buffered saline system -PBS-) is not always supplemented with vitamins or minerals [14]. In contrast, the concentration of the inoculum of eutrophic systems is lower $(\sim 1 \% \mathrm{w} / \mathrm{v})$ but a basal medium (usually basal culture medium -BCM-) fortified with bile salts, yeast extract and peptone is used [14]. 
It has been observed that differences in the nutritional availability in fermentation systems account for discrepancies in the development of the microbiota even in the presence or absence of a prebiotic [14-16]. Part of the media's modulatory effect is hypothesized to be derived from differences in the availability of bile salts and peptides [14].

When studying obesity, the use of a proper medium has to be evaluated in order to properly reproduce the nutritional components to which the microbiota from obese subjects are exposed. Despite the efforts of closely simulating the Western diet, fats are not incorporated in the media for in vitro studies yet. In fact, to our knowledge, there is no in vitro research performed so far on the effect of fats on the composition and activity of the human gut microbiota. Many of these studies focused on testing primarily carbohydrates, also excluding research on the effect of proteins, a drawback that limits the evaluation of the impact of microbe and diet interaction. In an average Western diet, about $15.4 \%$ and $32.8 \%$ of the energy intake comes from protein and fat, respectively [17]. Another important aspect to take into consideration is the concentration of the test compound to be evaluated. Short-term studies preferably use a high concentration of the test compound while long-term studies use relatively lower concentrations with the aim to detect more gradual changes such as the case of experiments in TIM-2 or SHIME [18].

\section{The use of a representative control}

A representative control for the in vitro investigation of the role of the gut microbiota in obesity is needed. Basically, two important points need to be addressed: one is reaching a consensus about what could be considered as a control in the experimental set up and the other one about the complexity of the media which is provided to the microbiota to grow. First, part of the inconsistency in the results of testing the effect of prebiotics in batch fermentations may come from the differences in the control used [14]. As explained by Long et al. [14], some studies regard samples taken at the initial point $\left(\mathrm{t}_{0}\right)$ as the baseline control to measure the response of the microbiota to the different substrates. Others, use a negative control which is basically a parallel fermentation in which the tested substrate is not added [14]. However, the use of the last case is not recommended due to the fact that these fermentations present a minimal production of SCFA accompanied by minimal changes in $\mathrm{pH}$ which has been interpreted as an indicator of lack of fermentation [19]. Second, as previously mentioned, the preparation of a complex media which primarily contains polymerized carbon and nitrogen sources has been considered representative to stimulate the optimal growth of communities simulating those residing in the intestine in in vitro fermentation studies [20]. However, this does not reflect entirely the environment to which the gut microbiota 
from obese subjects is exposed. The microbiota from obese subjects has been exposed to a repeated high energy diet [21]. To our knowledge only one study has attempted to replicate the high energy content in the media composition simulating a Western diet. In this study, the authors also compared the administration of normal energy and low energy in the simulation of a child gut. In order to accurately simulate the different nutrient loads and dietary conditions, the composition of the media used in this study was based on an extensive investigation of dietary records from anorexic, normal weight and obese children [21].

\section{The importance of testing the effect of fats on gut microbiota}

Dietary fat supplies around $9 \mathrm{kcal} / \mathrm{g}$ of energy, more than double of what is contributed by carbohydrate or protein (estimated to be around $4 \mathrm{kcal} / \mathrm{g}$ ) [22]. The nutritional dietary lipid recommendations for adults is around $20-35 \%$ of daily calories ingested [23].

Nowadays it is widely acknowledged from animal studies that fat overconsumption leads to obesity [24]. However, it is unclear how much fat reaches the colon. Some initial studies have indicated that from a load of $70 \mathrm{~g} / \mathrm{day}, 3.5 \mathrm{~g}$ enters the large intestine [25]. Still, more research is needed in this area.

There is evidence that indicates that dietary fat has a larger effect on the modulation of the composition of the gut microbiota than on the development of obesity itself. Such evidence mainly comes from studies in mice and to our knowledge there are no in vitro studies focusing on this matter. For instance, de Wit et al. [26] observed that the overflow of fat to the distal intestine triggered changes in the composition of the microbiota in mice. Specifically, it may reduce diversity and increase the Firmicutes-to-Bacteroidetes ratio. In addition, the microbiota from mice fed with a high fat diet for 12 weeks showed a strong response in terms of composition but returned indistinguishably to baseline after 10 weeks of switching back to the normal diet when compared to the control [27]. Therefore, it has been suggested that the response of the microbiota to fat exposure can be reversible indicating diet as a treatment for obesity [28]. Still, some studies indicate that the response may also depend on the specific diet composition as observed in conventional and germ-free mice [29].

\section{Mechanics of a dynamic environment}

The simulation of the mechanically dynamic environment characteristic for the human intestine, which includes intraluminal fluid flow and peristaltic motions, is poorly simulated in the current available in vitro continuous models of the human 
gut [30]. However, though limited, there are a number of models where at least shear forces are applied from peristaltic to stirring motions.

Shear forces are a key factor regulating bacterial gene expression and physiology, stress resistance, and adhesion of bacteria to the gut wall [31-34]. In general, shear stress (stirring) is applied in a bioreactor with the aim of increasing oxygen transfer in the media. In such case agitation speed has been found to be crucial for achieving high yields of enzyme production [35]. However, current models of the human gut solely aim to simulate the luminal colon. In an anaerobic system as such, the stirring of the culture media is performed with the purpose of homogenizing the suspension to guarantee that heat and food distribution is even in the system. The application of shear forces in an in vitro system simulating the human gut becomes crucial when it comes to the integration of cell lines in the experimental design. The cells in the human intestinal epithelial layer are exposed to rhythmic mechanical deformations originating from the peristaltic motion of the intestine [30]. Such conditions promote the differentiation of the cells and shape the height and polarization of these cells [30], which are factors that are important in microbe-host interaction.

\section{Redox potential}

The redox potential has been defined as the predisposition for a compound to gain electrons [36]. The interpretation of values measuring the redox potential in the media where cells are found can be used as an indicator of intracellular metabolism in which redox balance and electron transfer play an important role [36]. Therefore, redox potential has been considered as a sensitive tool to provide information about subtle changes in intracellular metabolism [36].

Environmental factors that affect the redox potential in a fermentation process include anaerobic conditions, dissolved oxygen, temperature and ratio of oxidizing to reducing compounds (represented most of the time by nutritional substrates provided in the medium, such as cysteine) [36-38]. These factors form part of the typical stress conditions to which the gut microbiota is exposed after the transfer from human gut to in vitro model.

Biologically speaking, many functions of the cells are affected by fluctuating redox potential values. These include altered gene expression, enzyme synthesis (directly affecting signal sensing and transduction) and metabolic profiles [39-41]. Therefore, it is important to reach homeostasis in terms of redox potential in the gut model to optimally simulate in vivo conditions. Such homeostasis is crucial for an optimal intracellular metabolism in the cells stimulating growth of these bacteria and their survival. 


\section{Anaerobiosis}

Linked to redox-potential is maintenance of physiological oxygen gradient concentration values, which is also important for proper simulation of the human gut. This implies a high level of complexity because oxygen is supplied by host tissue which consequently explains the higher oxygen content found close to the mucosal surface when compared to the anaerobic lumen [42], and the existence of an oxygen gradient along the radial axis of the gut. At present it is unclear whether there also is a longitudinal gradient along the proximal to distal gut.

Many in vitro models are restricted to the simulation of the colonic lumen where $\mathrm{N}_{2}$ is continuously flushed in order to maintain an $\mathrm{O}_{2}$-free atmosphere [11$13,43,44]$. However, an anaerobic atmosphere can also be established by initially flushing with $\mathrm{N}_{2}$ and then be kept by the usual gases produced by the fermentative activity of the microbiota as suggested by Feria-Gervasio et al. [45]. According to the authors, this method is believed to represent in vivo conditions better since it facilitates the action of hydrogenotrophs and decreases the $\mathrm{H}_{2}$ pressure. This could offer the advantage of studying reductive acetogens, sulphate reducing prokaryotes and methanogens at a higher resolution [45]. Furthermore, bacteria utilizing hydrogen are needed to prevent that fermentation reactions are blocked [46], this may be important in obesity research. For instance, it has been observed that propionate production increases by the inhibition of methanogens which are the main hydrogen utilizers [47].

$\mathrm{pH}$

In in vitro systems, $\mathrm{pH}$ has been found to influence the total amount of SCFA produced by the microbiota and their ratio. Furthermore, previous studies indicate that gut bacteria differ in their growth performance in response to changes in the $\mathrm{pH}$ of the media [48]. Such response, in terms of susceptibility or tolerance, could be directly associated with specific groups, including Bacteroides [48].

Examples of the effect of $\mathrm{pH}$ on the development of in vitro cultures include the study by Rajilic-Stojanovic et al. [49] who observed that the total amount of SCFA were lower for fermentations at $\mathrm{pH} 5.8$ when compared to $\mathrm{pH} 6.4$ and 7.0. Additionally, not only the final amounts were found to be affected but also the different molar ratios and diversity of the microbiota [49]. In the same manner, Walker et al.[48] observed substantial butyrate production in a static in vitro system using human microbiota fed with a polysaccharide composite and maintained at $\mathrm{pH}$ 5.5. However, when all conditions were maintained with the exception of $\mathrm{pH}$, which was increased to 6.5 , the authors observed a drastic drop in the concentration of butyrate accompanied with a substantial decreased of some bacteria (mainly Roseburia spp.). This emphasizes the fact that even a one-unit 
shift in $\mathrm{pH}$ shows a significant effect on metabolic profiles produced by the microbiota.

As mentioned earlier colonic $\mathrm{pH}$ varies as a function of host dietary intake [50]. Therefore, in view of the importance of reproducing a dense and active microbiota which will be physiologically relevant to understand its role in health and disease, it is crucial to set a proper $\mathrm{pH}$ value in the in vitro fermentation system when simulating obese subjects whose consumption of fermentable carbohydrates may vary when compared to lean subjects.

\section{Transit time}

Transit time is a physiological parameter that defines the time frame for the fermentation of a substrate in the colon [46]. Small intestinal transit time has been considered to play an important role in weight balance since it determines how much of the nutrients from the diet can be absorbed [51]. Therefore, a prolonged transit time has been suggested to promote a higher absorption and consequently the promotion of weight gain [51]. In contrast, a short transit time may be interpreted as a shorter time for absorption and it probably leads to weight loss or weight maintenance [51].

Obese subjects are part of the population where transit time is believed to be different when compared to other groups [52-55]. Furthermore, differences in transit time have shown to be dependent on the energy content of meals. For instance, low and high energy meals clearly differed in the small intestinal transit time in obese subjects (64 and 123 min, respectively) [56].

Despite that a large part of nutrients are absorbed in the small intestine, differences in transit time in this part of the tract influence the substrates which are finally available in the colon for both fermentation and absorption. This can also have an impact on host energy balance.

Average transit time in the colon of a healthy subject has been reported to range from 12 to $24 \mathrm{~h}$ [57]. Unfortunately, studies addressing colonic motility in obesity are limited [51]. However, evidence indicates a delayed total colonic transit time in obese children when compared to lean [54].

With respect to in vitro fermentation systems, the modification of the retention time has previously been shown to have an effect on the establishment of certain types of bacteria together with changes in their metabolic activities [58]. This is explained by the fact that substrate availability highly depends on retention time, and the growth rate of bacteria is based on how fast microorganisms are able to metabolize the available substrate and compete with others [58]. For instance, changes in retention time have been shown to significantly affect quantities of 
lactobacilli and bifidobacteria [45], carbohydrate degradation patterns [12,59] and production of specific metabolites such as butyrate $[60,61]$.

Special emphasis in in vitro models simulating the human gut in obesity has therefore also to be done with respect to the correlation between transit time and the reciprocal dilution rate as suggested by Cinquin et al.[43].

\section{Future perspectives}

The next step to proceed in the area of research described in this thesis is to determine the number of donors which could be used in the preparation. This makes it feasible in finding a representative inoculum to perform a wide range of in vitro studies for food and pharmaceutical studies. Further experiments are recommended to i) test the optimal time and alternatives for thawing as e.g., discussed by Hamilton et al.[62] who tested thawing using an ice-bath, ii) study the extent of the effects of preparing a human fecal inoculum on in vitro experiments fermenting specific substrates and iii) determine the effect of different treatments on microbial enzyme activities and gas production.

Future research should also be focused on improving the standard operating procedures of the TIM-2 system. This includes the inoculation of the model, the stabilization during the propagation of the inoculum during the first hours in the system, the $\mathrm{pH}$ chosen for the fermentation protocol, to name a few. Furthermore, studies including in vivo tests are suggested to explore the similarities in the response of diet in different individuals.

Another interesting area to explore in the (in vitro) study of human obesity is the investigation of the role of methanogens in energy balance. Although the prevalence of methanogens in the human gut has not been directly related with gender, it is believed to be affected by age, diet, geography and host genetic background [63]. In obesity, the study of archaeal species might be of interest due to the hypothesis that hydrogen transfer between bacteria and archaea may optimize the uptake of energy in individuals by increasing the production of SCFA [64].

The potential influence of methanogens in energy balance has been corroborated after finding an elevated presence of archaea in anorexic patients, speculated to be due to an adaptation of the microbiota with the aim to optimally use the low calorie diet ingested by the anorexic host. In obese and type 2 diabetic patients an enrichment in archaea has also been observed $[65,66]$. However, findings about the abundance of archaea after a diet intervention in obese subjects are contradictory. Methanogenic archaea did not show a significant change during a controlled diet in 
overweight men [67] while it was found to be more prevalent (together with Akkermansia) after a weight reduction intervention [68].

In vitro models of the human gut are of great potential to study the impact of methanogens in the dynamics of a gut bacterial community since the establishment of $M$. smithii in conventionalized rodents with human gut microbiota has failed $[69,70]$ while it has shown to be successful in an in vitro model [71]. 


\section{References}

1. World Health Organization (WHO) (2015) Obesity and overweight. Fact sheet No $311 \mathrm{http}: / / \mathrm{www}$. who.int/mediacentre/factsheets/fs311/en/

2. Aguirre M, Venema K (2015) Does the Gut Microbiota Contribute to Obesity? Going beyond the Gut Feeling. Microorganisms 3: 213.

3. Petrof EO, Claud EC, Gloor GB, Allen-Vercoe E (2013) Microbial ecosystems therapeutics: a new paradigm in medicine? Benef Microbes 4: 53-65.

4. Petrof EO, Gloor GB, Vanner SJ, Weese SJ, Carter D, et al. (2013) Stool substitute transplant therapy for the eradication of Clostridium difficile infection: 'RePOOPulating' the gut. Microbiome 1: 3 .

5. Englyst HN, Cummings JH (1986) Digestion of the carbohydrates of banana (Musa paradisiaca sapientum) in the human small intestine. Am J Clin Nutr 44: 42-50.

6. Englyst HN, Cummings JH (1987) Digestion of polysaccharides of potato in the small intestine of man. Am J Clin Nutr 45: 423-431.

7. Macfarlane G, Gibson G (1994) Metabolic activities of the normal colonic flora. In: Gibson SAW, editor. Human Health. London: Springer-Verlag. pp. 17-52.

8. Macfarlane G, Gibson G (1995) Microbiological aspects of the production of shortchain fatty acids in the large bowel. Physiological and clinical aspects of short-chain fatty acids: 87-105.

9. Gibson GR (1999) Dietary modulation of the human gut microflora using the prebiotics oligofructose and inulin. J Nutr 129: 1438S-1441S.

10. Macfarlane GT, Gibson GR, Cummings JH (1992) Comparison of fermentation reactions in different regions of the human colon. J Appl Bacteriol 72: 57-64.

11. Molly K, Vande Woestyne M, Verstraete W (1993) Development of a 5-step multichamber reactor as a simulation of the human intestinal microbial ecosystem. Appl Microbiol Biotechnol 39: 254-258.

12. Macfarlane GT, Macfarlane S, Gibson GR (1998) Validation of a Three-Stage Compound Continuous Culture System for Investigating the Effect of Retention Time on the Ecology and Metabolism of Bacteria in the Human Colon. Microb Ecol 35: 180-187.

13. Minekus M, Smeets-Peeters M, Bernalier A, Marol-Bonnin S, Havenaar R, et al. (1999) A computer-controlled system to simulate conditions of the large intestine with peristaltic mixing, water absorption and absorption of fermentation products. Appl Microbiol Biotechnol 53: 108-114.

14. Long W, Xue Z, Zhang Q, Feng Z, Bridgewater L, et al. (2015) Differential responses of gut microbiota to the same prebiotic formula in oligotrophic and eutrophic batch fermentation systems. Sci Rep 5: 13469.

15. Rycroft CE, Jones MR, Gibson GR, Rastall RA (2001) A comparative in vitro evaluation of the fermentation properties of prebiotic oligosaccharides. $J \mathrm{Appl}$ Microbiol 91: 878-887. 
16. Wang X, Gibson GR (1993) Effects of the in vitro fermentation of oligofructose and inulin by bacteria growing in the human large intestine. J Appl Bacteriol 75: 373-380.

17. Cordain L, Eaton SB, Sebastian A, Mann N, Lindeberg S, et al. (2005) Origins and evolution of the Western diet: health implications for the 21 st century. Am J Clin Nutr 81: 341-354.

18. Van den Abbeele P, Venema K, Van de Wiele T, Verstraete W, Possemiers S (2013) Different human gut models reveal the distinct fermentation patterns of Arabinoxylan versus inulin. J Agric Food Chem 61: 9819-9827.

19. Michel C, Kravtchenko TP, David A, Gueneau S, Kozlowski F, et al. (1998) In vitro prebiotic effects of Acacia gums onto the human intestinal microbiota depends on both botanical origin and environmental $\mathrm{pH}$. Anaerobe 4: 257-266.

20. Cummings JH, Macfarlane GT (1991) The control and consequences of bacterial fermentation in the human colon. J Appl Bacteriol 70: 443-459.

21. Payne AN, Chassard C, Banz Y, Lacroix C (2012) The composition and metabolic activity of child gut microbiota demonstrate differential adaptation to varied nutrient loads in an in vitro model of colonic fermentation. FEMS Microbiol Ecol 80: 608-623.

22. Wang DQ (2007) Regulation of intestinal cholesterol absorption. Annu Rev Physiol 69: 221-248.

23. Wang TY, Liu M, Portincasa P, Wang DQ (2013) New insights into the molecular mechanism of intestinal fatty acid absorption. Eur J Clin Invest 43: 12031223.

24. West DB, York B (1998) Dietary fat, genetic predisposition, and obesity: lessons from animal models. Am J Clin Nutr 67: 505S-512S.

25. Priebe MG, Vonk RJ, Sun X, He T, Harmsen HJ, et al. (2002) The physiology of colonic metabolism. Possibilities for interventions with pre- and probiotics. Eur J Nutr 41 Suppl 1: I2-10.

26. de Wit N, Derrien M, Bosch-Vermeulen H, Oosterink E, Keshtkar S, et al. (2012) Saturated fat stimulates obesity and hepatic steatosis and affects gut microbiota composition by an enhanced overflow of dietary fat to the distal intestine. Am J Physiol Gastrointest Liver Physiol 303: G589-599.

27. Zhang C, Zhang M, Pang X, Zhao Y, Wang L, et al. (2012) Structural resilience of the gut microbiota in adult mice under high-fat dietary perturbations. ISME $J$ 6: $1848-1857$.

28. Scott KP, Gratz SW, Sheridan PO, Flint HJ, Duncan SH (2013) The influence of diet on the gut microbiota. Pharmacol Res 69: 52-60.

29. Fleissner CK, Huebel N, Abd El-Bary MM, Loh G, Klaus S, et al. (2010) Absence of intestinal microbiota does not protect mice from diet-induced obesity. $\mathrm{Br} \mathrm{J}$ Nutr 104: 919-929. 
30. Kim HJ, Huh D, Hamilton G, Ingber DE (2012) Human gut-on-a-chip inhabited by microbial flora that experiences intestinal peristalsis-like motions and flow. Lab Chip 12: 2165-2174.

31. Nauman EA, Ott CM, Sander E, Tucker DL, Pierson D, et al. (2007) Novel quantitative biosystem for modeling physiological fluid shear stress on cells. Appl Environ Microbiol 73: 699-705.

32. Nickerson CA, Ott CM, Wilson JW, Ramamurthy R, LeBlanc CL, et al. (2003) Low-shear modeled microgravity: a global environmental regulatory signal affecting bacterial gene expression, physiology, and pathogenesis. J Microbiol Methods 54: 1-11.

33. Nilsson LM, Thomas WE, Sokurenko EV, Vogel V (2006) Elevated shear stress protects Escherichia coli cells adhering to surfaces via catch bonds from detachment by soluble inhibitors. Appl Environ Microbiol 72: 3005-3010.

34. Lebeer S, Verhoeven TLA, Perea Vélez M, Vanderleyden J, De Keersmaecker SCJ (2007) Impact of Environmental and Genetic Factors on Biofilm Formation by the Probiotic Strain Lactobacillus rhamnosus GG. Appl Environ Microbiol 73: 6768-6775.

35. Silva-Santisteban BOYp, Filho FM (2005) Agitation, aeration and shear stress as key factors in inulinase production by Kluyveromyces marxianus. Enzyme Microb Technol 36: 717-724.

36. Liu CG, Xue C, Lin YH, Bai FW (2013) Redox potential control and applications in microaerobic and anaerobic fermentations. Biotechnol Adv 31: 257-265.

37. Kjaergaard L (1977) The redox potential: Its use and control in biotechnology. Advances in Biochemical Engineering, Volume 7. Berlin, Heidelberg: Springer Berlin Heidelberg. pp. 131-150.

38. Chen X, Jiang S, Zheng Z, Pan L, Luo S (2012) Effects of culture redox potential on succinic acid production by Corynebacterium crenatum under anaerobic conditions. Process Biochem 47: 1250-1255.

39. Mason JT, Kim S-K, Knaff DB, Wood MJ (2006) Thermodynamic Basis for Redox Regulation of the Yap1 Signal Transduction Pathway. Biochemistry 45: 13409-13417.

40. Riondet C, Cachon R, Waché Y, Alcaraz G, Diviés C (2000) Extracellular Oxidoreduction Potential Modifies Carbon and Electron Flow in Escherichia coli. J Bacteriol 182: 620-626.

41. Vemuri GN, Altman E, Sangurdekar DP, Khodursky AB, Eiteman MA (2006) Overflow Metabolism in Escherichia coli during Steady-State Growth: Transcriptional Regulation and Effect of the Redox Ratio. Appl Environ Microbiol 72: 3653-3661.

42. Wilson M (2005) Microbial Inhabitants of Humans: Their Ecology and Role in Health and Disease: Cambridge University Press. 
43. Cinquin C, Le Blay G, Fliss I, Lacroix C (2004) Immobilization of infant fecal microbiota and utilization in an in vitro colonic fermentation model. Microb Ecol 48: 128-138.

44. Gibson GR, Cummings JH, Macfarlane GT (1988) Use of a three-stage continuous culture system to study the effect of mucin on dissimilatory sulfate reduction and methanogenesis by mixed populations of human gut bacteria. Appl Environ Microbiol 54: 2750-2755.

45. Feria-Gervasio D, Denis S, Alric M, Brugere JF (2011) In vitro maintenance of a human proximal colon microbiota using the continuous fermentation system P-ECSIM. Appl Microbiol Biotechnol 91: 1425-1433.

46. De Boever P, Wouters R, Vermeirssen V, Boon N, Verstraete W (2011) Development of a Six-Stage Culture System for Simulating the Gastrointestinal Microbiota of Weaned Infants. Microb Ecol Health Dis 13.

47. Demeyer D, Piattoni F, Mbanzamihigo L, Immig I, Nollet L (1997) Alternative hydrogen sink pathways in hindgut fermentation. Reprod Nutr Dev 37: 67-68.

48. Walker AW, Duncan SH, McWilliam Leitch EC, Child MW, Flint HJ (2005) pH and peptide supply can radically alter bacterial populations and short-chain fatty acid ratios within microbial communities from the human colon. Appl Environ Microbiol 71: 3692-3700.

49. Rajilic-Stojanovic M, Maathuis A, Heilig HG, Venema K, de Vos WM, et al. (2010) Evaluating the microbial diversity of an in vitro model of the human large intestine by phylogenetic microarray analysis. Microbiology 156: 32703281.

50. Duncan SH, Louis P, Thomson JM, Flint HJ (2009) The role of pH in determining the species composition of the human colonic microbiota. Environ Microbiol 11: $2112-2122$.

51. Mushref MA, Srinivasan S (2013) Effect of high fat-diet and obesity on gastrointestinal motility. Ann Transl Med 1: 14.

52. Dubois A, Gross HA, Ebert MH, Castell DO (1979) Altered gastric emptying and secretion in primary anorexia nervosa. Gastroenterology 77: 319-323.

53. Wright RA, Krinsky S, Fleeman C, Trujillo J, Teague E (1983) Gastric emptying and obesity. Gastroenterology 84: 747-751.

54. vd Baan-Slootweg OH, Liem O, Bekkali N, van Aalderen WM, Rijcken TH, et al. (2011) Constipation and colonic transit times in children with morbid obesity. J Pediatr Gastroenterol Nutr 52: 442-445.

55. Xing J, Chen JD (2004) Alterations of gastrointestinal motility in obesity. Obes Res 12: $1723-1732$.

56. Wilmshurst P, Crawley JC (1980) The measurement of gastric transit time in obese subjects using $24 \mathrm{Na}$ and the effects of energy content and guar gum on gastric emptying and satiety. Br J Nutr 44: 1-6. 
57. Guerra A, Etienne-Mesmin L, Livrelli V, Denis S, Blanquet-Diot S, et al. (2012) Relevance and challenges in modeling human gastric and small intestinal digestion. Trends Biotechnol 30: 591-600.

58. Child MW, Kennedy A, Walker AW, Bahrami B, Macfarlane S, et al. (2006) Studies on the effect of system retention time on bacterial populations colonizing a three-stage continuous culture model of the human large gut using FISH techniques. FEMS Microbiol Ecol 55: 299-310.

59. Macfarlane S, Quigley ME, Hopkins MJ, Newton DF, Macfarlane GT (1998) Polysaccharide degradation by human intestinal bacteria during growth under multi-substrate limiting conditions in a three-stage continuous culture system. FEMS Microbiology Ecology 26: 231-243.

60. Cummings JH, Hill MJ, Bone ES, Branch WJ, Jenkins DJ (1979) The effect of meat protein and dietary fiber on colonic function and metabolism. II. Bacterial metabolites in feces and urine. Am J Clin Nutr 32: 2094-2101.

61. Lewis SJ, Heaton KW (1997) Increasing butyrate concentration in the distal colon by accelerating intestinal transit. Gut 41: 245-251.

62. Hamilton MJ, Weingarden AR, Sadowsky MJ, Khoruts A (2012) Standardized frozen preparation for transplantation of fecal microbiota for recurrent Clostridium difficile infection. Am J Gastroenterol 107: 761-767.

63. Gaci N, Borrel G, Tottey W, O'Toole PW, Brugere JF (2014) Archaea and the human gut: new beginning of an old story. World J Gastroenterol 20: 1606216078.

64. DiBaise JK, Frank DN, Mathur R (2012) Impact of the Gut Microbiota on the Development of Obesity: Current Concepts. Am J Gastroenterol Suppl 1: 2227.

65. Zhang H, DiBaise JK, Zuccolo A, Kudrna D, Braidotti M, et al. (2009) Human gut microbiota in obesity and after gastric bypass. PNAS 106: 2365-2370.

66. Remely M, Dworzak S, Hippe B, Zwielehner J, Aumueller E, et al. (2013) Abundance and diversity of microbiota in type 2 diabetes and obesity. $J$ Diabetes Metab 2013.

67. Walker AW, Ince J, Duncan SH, Webster LM, Holtrop G, et al. (2011) Dominant and diet-responsive groups of bacteria within the human colonic microbiota. The ISME journal 5: 220-230.

68. Remely M, Tesar I, Hippe B, Gnauer S, Rust P, et al. (2015) Gut microbiota composition correlates with changes in body fat content due to weight loss. Beneficial microbes: 1-9.

69. Goodrich JK, Waters JL, Poole AC, Sutter JL, Koren O, et al. (2014) Human genetics shape the gut microbiome. Cell 159: 789-799.

70. Samuel BS, Gordon JI (2006) A humanized gnotobiotic mouse model of hostarchaeal-bacterial mutualism. Proc Natl Acad Sci U S A 103: 10011-10016. 
71. Tottey W, Gaci N, Borrel G, Alric M, O'Toole PW, et al. (2015) In-vitro model for studying methanogens in human gut microbiota. Anaerobe 34: 50-52. 


\section{Acknowledgments}

Thank you!

Muchas gracias!

Dankjewel! 
Completing this 4 year Phd project would not have been possible without the contribution of so many people who were involved directly or indirectly in this work.

First of all I would like to express my gratitude to my promotor Prof. dr. Koen Venema. Dear Koen, I had the opportunity to work with you since my master's internship and I appreciate your guidance and dedication to both of the projects.

I really enjoyed these 4 years of working together. I am happy to see that we worked in such nice synergy: proof of that is that despite the "delays" we had in our own planning we managed to finish the thesis before the official time and with so many chapters already published. As I told you once: I think that we made a very good promotor-student team. Thank you for your prompt reaction to anything that was needed for the project.

I also express my sincere gratitude to my other promotor Prof. dr. Fred Brouns. Dear Fred, we knew each other since I was enrolled in your master's program. Thank you for your guidance and hospitality back to that time. I really appreciate how involved you were on the program as well as your sincere interest that everybody had a very nice study time. Thanks for extending that help and concern during the supervision of the progress of my $\mathrm{PhD}$ project.

Thanks to the reading committee: Prof. dr. W.H.M. Saris, Prof. dr. E.E. Blaak, Prof. dr. J. Knol, dr. J. Penders and Prof. dr. M.Y Sanz Herranz for taking the time to evaluate my thesis.

My sincere thanks also go to the teams I worked within this project:

From TNO: the Pharmacokinetics \& Human studies in which I initially joined and later the Microbiology and Systems Biology group in which I completed my project. Special thanks to Marjorie, Annet, $\mathbf{R o b}^{2}$, Jan, Bart, Wendy, Mark, Tom, Guus, Jordy, Martien, Heleen, Marian, Anita and Michel for sharing their knowledge and support. I would like to express my special appreciation to my colleagues Irene, Mariska, and Harm for bringing a gezellige atmosphere to our room. To Angelique for her dedication and effort in teaching me some Dutch and for the nice morning talks we had during this time as well as to Ronald for being so friendly and always speak with so much enthusiasm about the Latin-American culture $(-)$.

From TIFN: my sincere gratitude to the members of the GH004 team (microbiology group from Wageningen University -Javier, Gerben, Hauke and 
Erwin-, the industrial partners, technicians and my two $\mathrm{PhD}$ colleagues Christiane and I-Chiao) for the fruitful discussions during the expert meetings, WE days and/or papers in which we worked together.

I am also thankful to the IS-diagnostics: Dries, Paul, Anat, Linda and Malieka with whom I was able to work on two of the chapters of my thesis. Thanks for providing your insight and expertise that greatly assisted part of this research.

Thanks also to dr. Freddy Troost and dr. Daisy Jonkers who were my supervisors during my master's thesis which turned to be an appetizer from my $\mathrm{PhD}$ thesis. I appreciate working together with both of you and especially for growing in me that interest about the topic that finally motivated me to pursue this 4 year project.

I am immensely grateful with my PhD amigos: Dina, Ilse, Carlota, Sultan, Dulce and Yu. Thanks for the great moments that we shared together: lunches, dinners, trips, cocktails, movie night, disco night, house warming parties, birthday celebrations and TNO-PhD meetings. Dina: you were an example to me of a very disciplined, hard-working, friendly and full of positive energy person. Ilse: your tendency to dig into finding an answer to everything was always very inspiring. Carlota: no words to describe how much I appreciate you as a colleague and as a friend. I am very lucky of having shared this journey together and that our latin background was so helpful in making us enjoy every single moment of our $\mathrm{PhD}$.

A mis amigas Maira, Maribel, Diana, Vanessa, Nathalia, Carolina y Nadia: gracias por continuar una amistad de hace muchos años. Por mostrar que no hay barreras para mantenerse en contacto aún así no se tenga Facebook y nuestras profesiones nos hayan llevado a hacer cosas totalmente diferentes. Infinitas gracias porque cada vez que nos encontramos es como si el tiempo nunca hubiera pasado, ustedes fueron durante estos años una parte importante en mi vida como estudiante de doctorado : siempre brindándome mucha motivación, ejemplo y apoyo moral.

Simone: special thanks to you as well. Meeting you was a great experience and it is so nice that we have so much fun together. I feel very happy that we are almost neighbors and I hope that our friendship will continue growing... bigger than our avocado plant (RIP)...

Por último todo mi amor a mi familia y a Yoram en retorno como agradecimiento.

A mis padres muchas gracias por todo su apoyo y su cariño durante todo este tiempo. Estoy absolutamente convencida en que no hubiera llegado a lograr completar una tesis de doctorado sin todo el sacrificio que ambos han hecho por 
mí. Este título es fruto de ese esfuerzo colectivo y es también de ustedes mis queridos doctores: los amo.

A mis hermanos Mauricio y Carlos: por cuestiones del destino tuve la fortuna de estar acompañada de ustedes durante este proceso. Nuestros fines de semana juntos han sido los mejores de estos 4 años. Los amo y los admiro inmensamente, en muchos aspectos ustedes dos han sido mi ejemplo a seguir. Espero que podamos seguir cerca el uno del otro por mucho más tiempo.

Thank you Mariel for all the wonderful moments that we shared together and your support during my time not only as a $\mathrm{PhD}$ student but also during my masters study. I feel very lucky that during the years that I have lived in the Netherlands we just got closer and closer. I deeply feel proud of you: your sharp intelligence and your loving kindness make me feel great respect and admiration for you.

Gracias también a los niños más lindos del mundo: Emma y Samuel. Me han hecho sentir la tía más feliz de todos los tiempos. Acompañarlos a ustedes en sus primeros pasos, palabras, cumpleaños, navidades, etc., y compartir su pasión ya sea por el universo o por explorar el mundo con ayuda de mucha fantasía han sido parte de los momentos más dulces de mi vida. Ustedes fuera de darme amor me dieron inspiración para terminar este proyecto. Los amo hasta el infinito!

A mi querido Yoram muchas gracias por tu amor, tu paciencia, apoyo y generosidad. Por entender cada vez que tuve que trabajar los fines de semana y sobretodo por cocinar platos especiales para llenarme de mucha energía. Gracias por luchar por un día a día en que seamos felices y soñar en un futuro juntos. Yo también espero lo mismo $:$ :

\section{Marisol}


About the author 


\section{Curriculum vitae}

Marisol Aguirre Morales was born in Palmira, Colombia, on the $30^{\text {th }}$ of May 1986. In 2003 she graduated from secondary school at "El Sagrado Corazón de Jesus, hermanas Bethlemitas" in Palmira. In 2004 she started her bachelor in Food Engineering at Universidad Jorge Tadeo in Bogotá, Colombia. She finished her bacherol thesis entitled "Determination of the physicochemical, microbiological and functional parameters of broccoli (Brassica oleracea var. italica) packed in modified atmospheres during simulated transportation" under the supervision of Prof. dr. N.Y. Pineros in 2009 and graduated "cum laude". After her bachelor, she worked as a research assistant in the project: "Stabilization and potential uses of Colombian rice bran for industrial use without affecting its nutritional and functional quality" at Universidad Jorge Tadeo Lozano in the Faculty of Food Engineering. In 2010, after receiving the UM High Potential Scholarship granted by Maastricht University, she moved to Venlo, The Netherlands. There, she continued with the master Health Food Innovation Management from the same university. Her master thesis entitled "The role of gut microbiota in lean and overweight subjects" was performed in The Netherlands Organization for Applied Scientific Research (TNO), Zeist and was supervised by dr. K. Venema and dr. Daisy Jonkers. In September, 2012, she continued working on this research line and started her $\mathrm{PhD}$ also at Maastricht University, but developing her research activities at TNO, Zeist. Her $\mathrm{PhD}$ research was part of a large project embedded in the Gastrointestinal Health theme from the Top Institute of Food \& Nutrition (TIFN). The project was entitled "Molecular interactions of mucosal tissues, bacteria and fibers" (GH004) under supervision of Prof. dr. F. Brouns and Prof. dr. K. Venema.

Contact: aguirre_marisol@yahoo.com

LinkedIn: nl.linkedin.com/in/marisol-aguirre-66546440 


\section{List of publications}

Aguirre M, Eck A, Koenen ME, Savelkoul PHM, Budding AE, Venema K (2016) Diet drives quick changes in the metabolic activity and composition of human gut microbiota in a validated in vitro gut model. Research in Microbiology. 167: 114125 .

Aguirre M, Venema K (2015) The use of fecal samples for studying human obesity. Eur J Epidemiol 30(9):1067-9.

Aguirre M, Eck A, Koenen ME, Savelkoul PHM, Budding AE, Venema K (2015) Evaluation of an optimal preparation of human standardized fecal inocula for in vitro fermentation studies. J Microbiol Methods 117: 78-84.

Aguirre M, Venema K (2015) The art of targeting gut microbiota for tackling human obesity. Genes \& nutrition. 10 (4), 1-12

Aguirre M, Venema K (2015) Does the Gut Microbiota Contribute to Obesity? Going beyond the Gut Feeling. Microorganisms 3(2): 213-235.

Aguirre M, Jonkers DMAE, Troost FJ, Roeselers G, Venema K (2014) In Vitro Characterization of the Impact of Different Substrates on Metabolite Production, Energy Extraction and Composition of Gut Microbiota from Lean and Obese Subjects. PLoS ONE 9(11): e113864.

Aguirre M, Ramiro-Garcia J, Koenen ME, Venema K (2014) To pool or not to pool? Impact of the use of individual and pooled fecal samples for in vitro fermentation studies. J Microbiol Methods 107C: 1-7.

Leijdekkers AGM, Aguirre M, Venema K, Bosch G, Gruppen H, et al. (2014) In Vitro Fermentability of Sugar Beet Pulp Derived Oligosaccharides Using Human and Pig Fecal Inocula. Journal of Agricultural and Food Chemistry 62: 1079-1087 


\section{Submitted publications}

Aguirre M, Bussolo de Souza C, Venema K. (2016) The gut microbiota from lean and obese subjects contribute differently to the fermentation of arabinogalactan and inulin.

Aguirre M, Venema K. (2016) Challenges in simulating the human gut for understanding the role of the microbiota in obesity.

\section{In preparation}

Aguirre $\mathbf{M}^{*}$, Hermes $\mathrm{G}^{*}$, Ramiro-García $\mathrm{J}^{*}$, Ripken D, Kaiser D, Smidt H, Zoetendal E, Koenen ME, Venema K. Seeing is believing: a repeatable, robust and reproducible in vitro simulation of the human proximal colon.

Aguirre M, Caspers M, Ossendrijver M, Roeselers G, Ouwens A, Venema K. Dynamics of defined and complex microbial communities fermenting mucin in an in vitro simulation of the human gut. 